THE UNIVERSITY OF OKLAHOMA

GRADUATE COLLEGE

\title{
THE USE OF MICELLAR SOLUTIONS FOR NOVEL SEPARATION \\ TECHNIQUES
}

A DISSERTATION

SUBMITTED TO THE GRADUATE FACULTY

in partial fulfillment of the requirements for the

\author{
degree of \\ DOCTOR OF PHILOSOPHY \\ DISCLAIMER
}

\begin{abstract}
This report was prepared as an account of work sponsored by an agency of the United States Government. Neither the United States Government nor any agency thereof, nor any of their employees, makes any warranty, express or implied, or assumes any legal liability or responsibility for the accuracy, completeness, or usefulness of any information, apparatus, product, or process disclosed, or represents that its use would not infringe privately owned rights. Reference herein to any specific commercial product, process, or service by trade name, trademark, manufacturer, or otherwise does not necessarily constitute or imply its endorsement, recommendation, or favoring by the United States Government or any agency thereof. The views and opinions of authors expressed herein do not necessarily state or reflect those of the United States Government or any agency thereof.
\end{abstract}

$$
\text { By }
$$

\section{BRUCE LYNN ROBERTS}

Norman, Oklahoma 


\section{ACKNOWLEDGMENTS}

The author wishes to acknowledge the assistance of the following individuals and organizations in this work:

Dr. John F. Scamehorn, whose constant encouragement, endless patience, profound example and leadership (technical and political) enabled this author to complete this endeavor and experience significant personal growth.

Dr. Sherril D. Christian, whose technical assistance and guidance were invaluable, and whose views on economics and government were thought provoking.

Dr. Jeffrey H. Harwell, Dr. Robert L. Shambaugh and Dr. Roger Harrison for serving on my committee and for always being very accessible and open to discussion on many topics.

Dr. Hirotaka "HERO" Uchiyama and Dr. Edwin Tucker for the chlorinated organic compounds in an aqueous surfactant solution vapor liquid equilibrium data and the resulting solubilization constants from that data in Chapter 7. I also fondly remember going to what seemed like every used car lot in central Oklahoma in the dead of winter with daily highs of $18^{\circ}$ F to help Dr. HERO purchase his first car, a sporty white Pontiac Grand Am (although 10 years from now he will argue that it was not white but some other color). I would also like to say that Dr. Ed Tucker is really becoming an excellent engineer for a physical chemist, no matter how desperately he tries not to. It just goes to show how much a person can improve. 
This Dissertation is Dedicated to

My Loving (and extremely patient) Wife, Linda .

and Our Pets: Cueball, Mork, Mindy, and Norman. 
Yuefeng Yin for running a stripping apparatus and collecting the samples for the vacuum stripping of chlorinated hydrocarbons from a surfactant solution.

Chon Lin Lee for his analysis of column effluents for investigations discussed in Chapter 4.

Dr. Asmare Atalay for his timely help with getting a Hall detector and gas chromatograph operational for the analysis of halogenated organics.

Amadali "Alex" Tabatabai was very helpful by showing me how to use atomic adsorption for analysis of aqueous solutions for metal concentrations:

Mark "Dean" Yeskie for introducing me to vericolor slide film. It has saved so much time compared to the orthilith film and dying process.

$\mathrm{K}$ (pronounced like "Kay") Hudson and Larry Isley for providing assistance in the acquisition of various items required for this study and also for their always helpful spirit during many equipment fabrications.

Carolyn Lauron for doing such an outstanding job of grading homework for two semesters of Intro. to Engineering that I taught.

Tim Melton for his intriguing discussions on haz-ops as well as how bomb construction can be a recreational hobby.

There are a large number of associates who have added to this experience: Dr. Jeff Lopata, Cheryl Haskins (a foxy grader according to her students ), Achille "Lee Raveolli" Riviello, Bita "no secrets" Young, Laura (just turned 30) Wesson, Jerry (blue sky) Newman, Martina Dreyer, Nancy Gullickson, Dr. Dawlat El-Sayed, Dr. Timothy "T-Bone" Fitzgerald, Dr. Kevin Gering, Dr. Vinod Tuliani, and of course Dr. Robert "Bob" Dunn have all helped and I am grateful.

The CEMS department staff has been very gracious and just a superb group of individuals. Sherry Childress, Lou Dunn, Donna King, Rick 
Wheeler, Polly Dvorak, Mary Ann Drass and Margaret Williford could not have been more helpful, I thank you very much.

The authors appreciate the financial support of the Office of Basic Energy Sciences, Department of Energy, contract DE-FG05-87ER13678, Department of Energy grant No. DE-FG01-87FE61146, and National Science Foundation grant CBT 8814147, EPA grant R-817450-01-0, University of Nevada-Reno, contract 90-09, and an Applied Research Grant from the Oklahoma Centers for the Advancement of Science and Technology. In addition, they gratefully acknowledge the assistance of industrial sponsors of the Institute for Applied Surfactant Research, including Aqualon Company, Kerr-McGee Corporation, Sandoz Chemicals Corp., E. I. Du Pont de Nemours \& Co., Unilever, Inc., Union Carbide Corporation, Dow Chemical Company, ICI Chemicals, and Shell Development Company. 
Table of Contents

CHAPTER

1. INTRODUCTION. 1

1.1 Micellar-Enhanced Separation Processes .................6

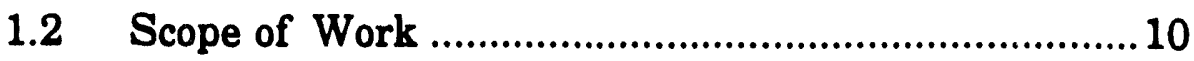

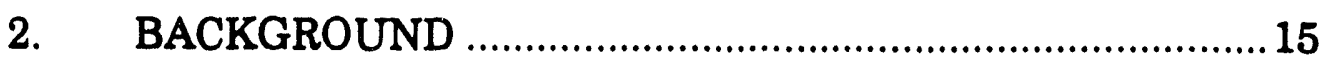

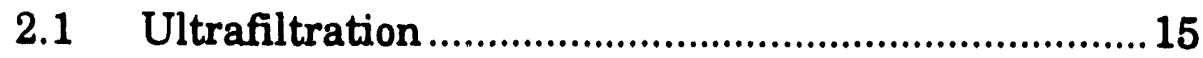

2.1.1 Ultrafiltration Membranes............................16

2.1.2 Concentration Polarization ............................ 18

2.1.3 Gel Polarization ..........................................20

2.1.4 Stirred Cell.................................................23

2.1.5 Spiral Wound Membrane System ...................25

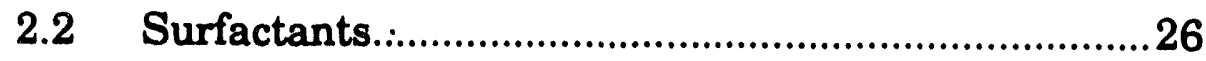

2.2.1 Micelle Formation and Structure ...................27

2.2.2 Models Describing Micelle Formation ............28

2.3 Solubilization of Dissolved Organic Molecules .........29

2.3.1 Solubilization Constant ...................................30

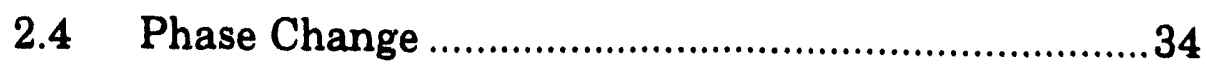


Table of Contents (Continued)

CHAPTER

2.5 Counterion-Níicelle Interaction 35

2.5.1 The Effect of Counterion Characteristics on Counterion Binding

2.5.2 Separation of Multivalent Counterions 37

2.5.3 Separation of Multivalent Ions 37

2.5.4 Oosawa Model Applied to MEUF 38

2.6 Activated Carbon 41

2.6.1 Granular Activated Carbon Beds .43

3. EXPERIMENTAL PROCEDURES...................................66

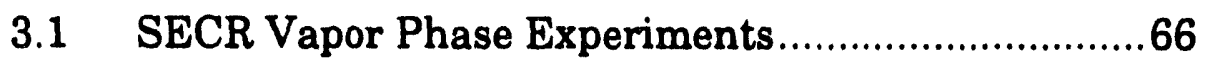

3.2 SECR Liquid Phase Experiments ...............................77

3.2.1 Dynamic Column Experiments ......................71

3.2.2 Static Equilibrium Experiments ....................73

3.3 Micellar-Enhanced Ultrafiltration ............................73

3.4 Vacuum Stripping of Chlorinated Hydrocarbons from Micellar Solutions...................................76

3.5 Vapor-Liquid Equilibrium Studies ...........................79 
Table of Contents (Continued)

CHAPTER

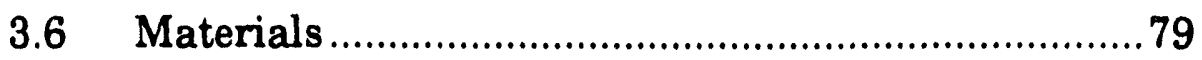

3.6.1 Sodium Dodecyl Sulfate...................................79

3.6.2 Dowfax 8390 and Dowfax 3B2 ……..................

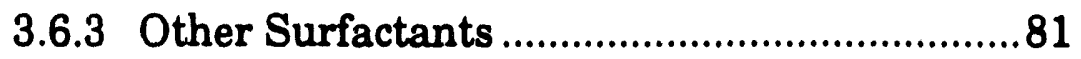

3.6.4 Target Organics Investigated ...........................

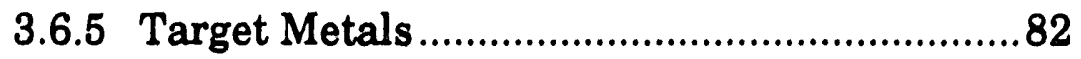

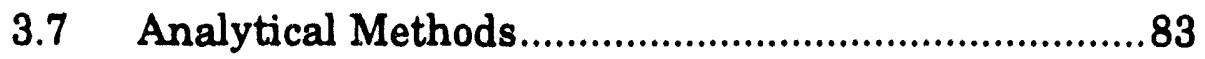

3.7.1 HPLC Analytical Methods..............................83

3.7.2 Gas Chromatography Analysis ......................91

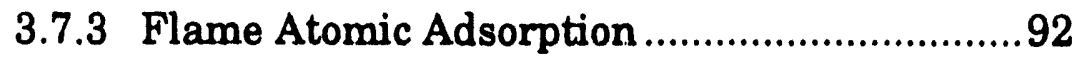

4. THE USE OF SURFACTANT-ENHANCED CARBON REGENERATION TO RECOVER VOLATILE ORGANICS FROM SPENT ACTIVATED CARBON 112

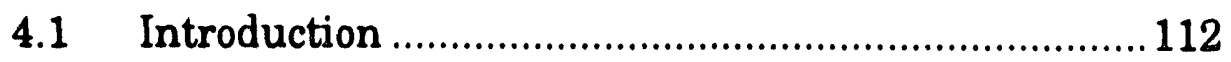

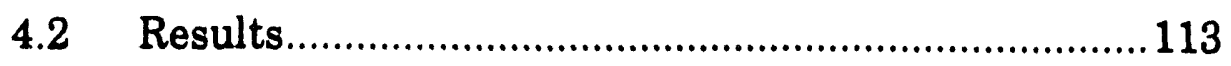

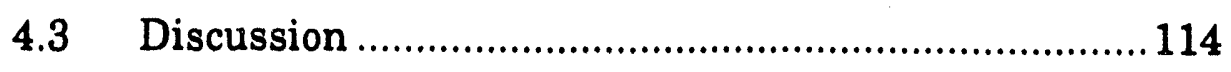

4.3.1 The Effect of Solute Loading ..........................114 
Table of Contents (Continued)

CHAPTER

4.3.2 The Effect of Multiple Cycle

115

4.3.3 The Effect of Solute Type

4.3.4 The Effect of Regenerant Solution Flow Rate

4.3.5 The Effect of Surfactant Concentration in Regenerant Solution

4.3.6 The Causes of Mass Transfer Limitation

4.3.7 The Water Flush Step.

4.4 Conclusions

5. USE OF SURFACTANT-ENHANCED CARBON REGENERATION TO REMOVE WATER SOLUBLE ORGANICS FROM SPENT ACTIVATED CARBON 126

5.1 Introduction 126

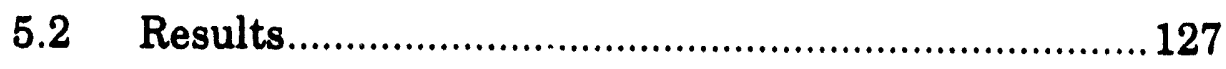

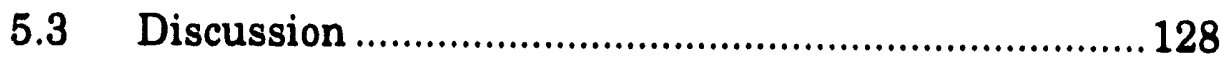

5.3.1 The Effect of Temperature on the Equilibrium Adsorption of CPC and SDS on Carbon 128

5.3.2 The Effect of Temperature On The Desorption of CPC 
Table of Contents (Continued)

$\begin{array}{lll}\text { CHAPTER } & \text { Page }\end{array}$

5.3.3 Dynamic Experiments

130

5.3.4 Other Considerations.....................................131

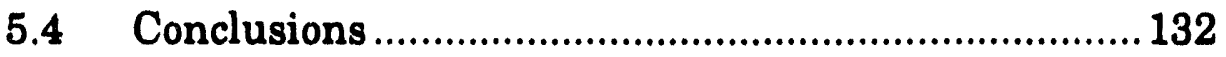

6. SCALE UP OF MICELLAR ENHANCED

ULTRAFILTRATION FOR THE RECOVERY

OF TARGET MULTIVALENT IONS AND

NONIONIC ORGANICS

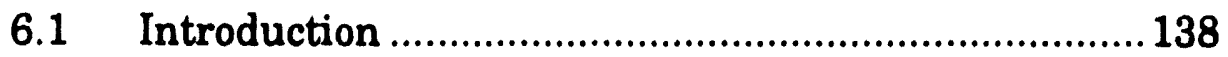

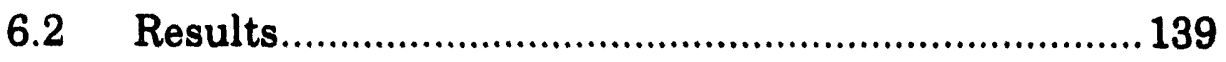

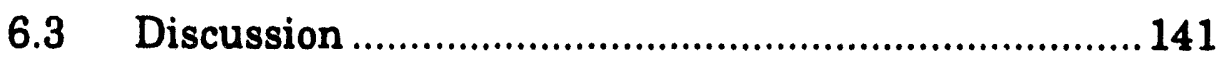

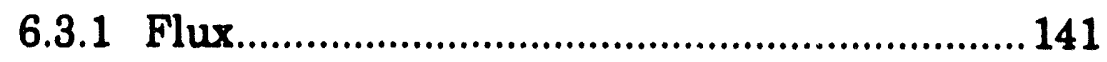

6.3.2 Comparison of Stirred Cell and Spiral Wound Relative Flux.

6.3.3 Comparison of Stirred Cell and Spiral Wound Permeate Concentrations for the 5,000 MWCO Membrane.

6.3.4 Comparison of SDS, CPC and TBP

Permeate Concentrations for Different

Spiral Wound Molecular Weight Cut

Off Membranes. 
Table of Contents (Continued)

CHAPTER

6.3.5 The Effect of Added Electrolyte on Surfactant Permeate Concentration.

6.3.6 Comparison of Spiral Wound, Stirred Cell and Semiequilibrium Dialysis Systems..........146

6.4 Conclusions

7. EVALUATION OF THE APPLICATION OF MEUF FOR THE RECOVERY OF VOLATILE CHLORONATED HYDROCARBON COMPOUNDS FROM GROUNDWATER.

7.1 Introduction 168

7.2 Process Flow Diagram to Remove CHC from Water. 169

7.3 Results and Discussion. 171

7.3.1 Separation of TCE from Water in MEUF

Units 171

7.3.1.1 General Considerations.............171

7.3.1.2 Flux ............................................174

7.3.1.2 Separation Efficiency ..................176

7.3.1.4 Spiral Wound Ultrafiltration Unit Results 180

7.3.2 Vacuum Stripping 181

7.3.3 Process Design Calculations and Economics.. 185 
Table of Contents (Continued)

CHAPTER

7.3.3.1 Material Balance Calculations.............185

7.3.3.2Economic Evalưation of Process...........187

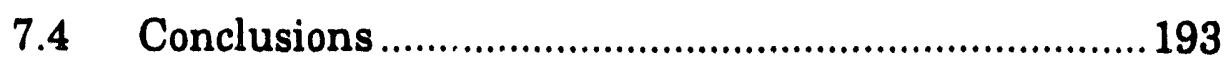

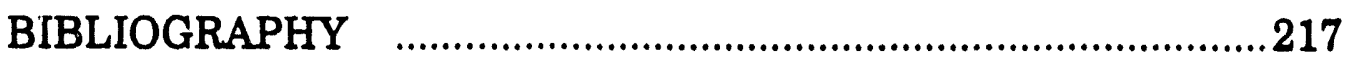

APPENDIX A 
List of Tables

TABLE

III.1 Major Equipment Used for SECR Investigations. 68

III.2 General Information About Surfactants Investigated..........94

III.3 General Information About Target Organics Investigated .95

III.4 General Information About Target Metals Investigated.....96

III.5 HPLC Equipment Used to Evaluate Samples for Ionic Compounds .....................................................................84

III.6 Solvents and Mode of Operation of HPLC for Analysis of Ionic Compounds 85

III.7 HPLC Equipment Used to Evaluate Surfactants and Target Organics with Chromophores

III.8 Solvents and Mode of Operation of HPLC for Analysis of Surfactants and Target Organics with Chromophores 88

VI.1 Removal of Various Target Organic Compounds from Water Using MEUF from Stirred-Cell Experiments

VII.1 Summary of the Effects of some Variables on the Sum of the Volumes of the Retentate Streams Divided by the Volume of Fresh Feed

VII.2 MEUF Data for TCE Using DOWFAX 8390 at Various Temperatures with and without Added Salts 
List of Tables (Continued)

TABLE

Page

VII.3 MEUF Data for PCE in a Mixture with TCE and DCM Using DOWFAX 8390 at Various Temperatures ...... 196

VII.4 MEUF Data for TCE in a Mixture with PCE and DCM Using DOWFAX 8390 at Various Temperatures .......197

VII.5 MEUF Data for DCM in a Mixture with PCE and TCE Using DOWFAX 8390 at Various Temperatures

VII.6 Results of Vacuum Stripping of TCE from a $0.2 \mathrm{M}$ DOWFAX 8390 Surfactant Solution

VII.7 Results of Vacuum Stripping of Mixtures of PCE, TCE, and DCM from a $0.25 \mathrm{M}$ Solution of DOWFAX 8390

VII.8 Results of Material Balance Calculations on MEUF Process for Trichloroethylene.

VII.9 Results of Material Balance Calculations on the MEUF Process for $1,1,1$, Trichloroethane

VII.10 Results of Material Balance Calculations on MEUF Process for Tetrachloroethylene. 203 


\section{List of Figures}

FIGURE

Page

I.1 Illustration of a Target Organic Solubilized in the Interior of a Micelle and a Divalent Metal Ion Electrostatically Bound to the Surface of a Micelle

I.2 Mlustration of MEUF Applied to the Removal of a Target Organic and Target Multivalent Cationic Metal in an Aqueous Solution 12

I.3 Ilustration of the SECR Concept ........................................13

I.4 Illustration of the Three Basic Steps Required for the Application of SECR

II.1 Overview of a Variety of

Separation Techniques, the Range of Separation and the Primary Factor Affecting the Separation ....44

II.2 Characteristics of Common Membrane

Separation Processes

II.3 The Relationship of the Size of Common Materials and Approximate Molecular Weights to the Range of Application for Pressure Driven Membrane Separation Processes

II.4 Mlustration of the Concept of Concentration Polarization with Gel Polarization of Macromolecules and Colloids During the Ultrafiltration Process. 
List of Figures (Continued)

FIGURE

II.5 Illustration of Stirred Cell and Major Components 48

II.6 Operational Characteristics of Stirred Cell

II.7 Illustration of the Geometry and Major Components of a Spiral Wound Ultrafiltration Membrane

II.8 Operation Characteristics of Spiral Wound Membrane

II.9 Illustration of a Surfactant Monomer and the Hydrophobic andHydrophilic Regions 52

II.10 Relationship of Total Surfactant Concentration, Monomer Concentration and Critical Micelle Concentration

II.11 Typical Micelle Composed of Anionic Monomers in an Aqueous Solution

II.12 Regions Where Different Types of Organic Solutes Solubilize within a Surfactant Micelle .55

II.13 Solubilization Location of Benzene Between the Hydrophilic Groups of Polyethoxylated Nonionic Surfactant Micelle 56

II.14 Maximum Additivity Method for Determination of Solubilization Constant.

II.15 Semiequilibrium Dialysis Cell Used for Solubilization Constant Determination 
List of Figures (Continued)

FIGURE

II.16 The Partition of Organic Solutes Between the Micellar Phase and the Solvent in Micellar Mobile Phase Chromatography for the Measurememt of the Solubilization Constant. 59

II.17 Counterion Adsorption at the Micelle-Aqueous Interface ...60

II.18 Counterion Concentration Profile Extending from the Micelle Surface

II.19 Ilustration of Stern Model of the Electrical Double Layer.62

II.20 Separation of Multivalent Counterions in MEUF.

II.21 Some Different Modes of Adsorption at the Solid-Liquid Interface

II.22 The Depletion of the Adsorptive Capacity of a Granular Activated Carbon Bed .65

III.1 Apparatus for Adsorption of the Target Organic

Solute on Activated Carbon

III.2 Apparatus for Water Flush to Wet Activated Carbon .........98

III.3 Apparatus for Micellar Flood to Solubilize and

Remove the Target Organic Solute

III.4 Apparatus for Water Flush to Remove Residual Surfactant in SECR.................................................100

III.5 Apparatus for Drying of the Activated Carbon Bed 101 
FIGURE

III.6 Apparatus for Vacuum Application to the Carbon Bed for the Prevention of Foam Formation Upon Introduction of the Surfactant Solution into the Bed 102

III.7 Apparatus for the Adsorption of Pyridinium Chloride. 103

III.8 Apparatus for the Water Flush of the Column Void

Volume and Desorption of Surfactant from Carbon 104

III.9 Configuration for the Adsorption of Tert-Butylphenol on the Carbon Bed from Water 105

III.10 Pilot Plant for the Micellar-Enhanced Ultrafiltration Process. 106

III.11 Mlustration of the Major Components of an

Ultrafiltration Spiral Wound Membrane 107

III.12 Vacuum Stripping Apparatus............................................ 108

III.13 Structures of DOWFAX Surfactants .................................... 109

III.14 Structures of Surfactants............................................. 110

III.15 Structures of Target Organic Solutes...............................111

IV.1 Effect of Toluene Loading on the Recovery of Toluene........119 
List of Figures (Continued)

FIGURE

IV.2 Effect of Amyl Acetate Loading on the Recovery of Amyl Acetate

IV.3 Effect of Surfactant Solution Flow Rate on the Recovery of Toluene

IV.4 Effect of Surfactant Solution Flow Rate on the Recovery of Amyl Acetate.

IV.5 Effect of Surfactant Concentration on the Recovery of Toluene.

IV.6 Effect of Surfactant Concentration on the Recovery of Amyl Acetate.

IV.7 Effect of Water Flow Rate on the Recovery of Residual Surfactant

V.1 Adsorption of Cetyl Pyridinium Chloride on Activated Carbon

V.2 Adsorption of Sodium Dodecyl Sulfate on Activated Carbon

V.3 Breakthrough Curves for Tert-Butylphenol on Surfactant Regenerated Carbon and Virgin Carbon 135

V.4 Percentage Recovery of Cetyl Pyridinium Chloride from the System During the Water Flush Step 
List of Figures (Continued)

FIGURE

Page

V.5 Percentage Recovery of Cetyl Pyridinium Chloride from the Carbon Surface During the Water Flush Step ... 137

VI.1 Relative Permeate Flux Using CPC and 5,000 MWCO Spiral Wound Membrane.

VI.2 Permeate CPC Concentrations Using CPC and 5,000 MWCO Spiral Wound Membrane

VI.3 Permeate TBP Concentration Using CPC and 5,000 MWCO Spiral Wound Membrane

VI.4 Comparison of Stirred Cell and Spiral Wound Membrane Relative Permeate Fluxes for a 5,000

MWCO Membrane

VI.5 Comparison of Stirred Cell and Spiral Wound

Ultrafiltration Membrane Performance, CPC, 5,000 MWCO Membrane

VI.6 Comparison of Stirred Cell and Spiral Wound

Membrane Performance, TBP, 5,000

MWCO Membrane 153

VI.7 Comparison of CPC Permeate Concentration for Different Molecular Weight Cut Off Spiral Wound Membranes. 154

VI.8 Comparison of TBP Concentrations for Different

Molecular Weight Cut off Spiral Wound

Membranes 155 
List of Figures (Continued)

FIGURE

Page

VI.9 Comparison of SDS Permeate Concentration for Different Molecular Weight Cut Off Spiral

Wound Membranes...

VI.10 Comparison of TBP Permeate Concentration for Different Molecular Weight Cut Off Spiral

Wound Membranes.

VI.11 Comparison of the SDS Permeate Concentrations at Different Copper Feed Concentrations for

a 10,000 WMCO Spiral Wound Membrane 158

VI.12 SDS Permeate Concentration for Retentate Solutions Composed of TBP and $\mathrm{Cu}+2$ and Using a Spiral Wound 20,000 MWCO Membrane 159

VI.13 Permeate Concentration of CPC with and without Added $\mathrm{CrO}_{4}^{-2}$ Using a 5,000 MWCO and a 20,000 MWCO Spiral Wound Membrane

VI.14 Ratio of $\mathrm{Cu}+2$ Concentration to SDS Concentration for the 10,000 MWCO Spiral Wound Membrane Study

VI.15 Ratio of $\mathrm{Cu}+2$ Concentration to SDS Concentration for the 20,000 MWCO Spiral Wound Membrane Study

VI.16 Ratio of $\mathrm{CrO}_{4}^{-2}$ Concentration to CPC Concentration for the 20,000 MWCO Spiral Wound Membrane Study 
FIGURE

VI.17 Comparison of the permeate $\mathrm{Cu}+2$ Concentration for Stirred Cell and Spiral Wound Membranes, 10,000 MWCO

VI.18 Comparison of the Percent Rejection of $\mathrm{Cu}+2$ for Spiral

Wound and Stirred Cell, 10,000 MWCO

Membranes

VI.19 Comparison of the Permeate $\mathrm{CrO}_{4}^{-2}$ Concentration

for a Semiequilibrium Dialysis Cell and Spiral

Wound Membrane

VI.20 Comparison of the Percent Rejection of $\mathrm{CrO}_{4}^{-2}$ for Spiral

Wound and Semiequilibrium Dialysis

Membranes

VII.1 Process Flow Diagram for Recovery of TCE from

Groundwater Using MEUF in a Four Stage

Process.

VII.2 Process Flow Diagram for Recovery of TCE from

Groundwater Using MEUF in a Four Stage

Process and a Single Stripper. 205

VII.3 The Relative Permeate Flux for Several Surfactants for Spiral Wound and Stirred Cell Studies using 10K MWCO Ultrafiltration Membranes.

VII.4 The Solubilization Constant of TCE in 0.05 M DOWFAX 8390 at Different Mole Fractions of TCE in the Micelles 
List of Figures (Continued)

FIGURE

VII.5 The Solubilization Constant of PCE in 0.05 M DOWFAX 8390 at Different Mole Fractions of PCE in the Micelles

VII.6 The Solubilization Constant of TCEA in 0.05 M DOWFAX 8390 at Different Mole Fractions of TCEA in the Micelles

VII.7 The Solubilization Constant of TCE in 0.05 M Surfactant Solution Composed of 0.9 Mole Fraction DOWFAX 3B2 and 0.1 Mole Fraction STEDBAC at Different Mole Fractions of TCE in the Micelles.

VII.8 The Solubilization Constant of TCE in 0.05 M CPC Surfactant Solutions at Different Mole Fractions of TCE in the Micelles $20^{\circ} \mathrm{C}$ and $30^{\circ} \mathrm{C}$

VII.9 The Solubilization Constant of PCE in $0.05 \mathrm{M} \mathrm{CPC}$ at Different Mole Fractions of PCE in the Micelles ......212

VII.10 The Cost of DOWFAX 8390 Lost in the Final Permeate Stream

VII.11 The Permeate Flux for DOWFAX 8390 Using a 500 MWCO

Spiral Wound UItrafiltration Membrane.

VII.12 The Permeate Concentration for DOWFAX 8390 using a 500 MWCO Spiral Wound Ultrafiltration Membrane. 


\section{List of Figures (Continued)}

FIGURE

Page

VII.13 The Effect of the Number of Transfer Units and Stripping

Factor on the Reduction Factor for TCE...................216 


\section{ABSTRACT}

Surfactant based separation techniques based on the solubilization of organic compounds into the nonpolar interior of a micelle or electrostatic attraction of ionized metals and metal complexes to the charged surface of a micelle were studied in this work.

Micellar solutions were used to recover two model volatile organic compounds emitted by the printing and painting industries (toluene and amyl acetate) and to investigate the effect of the most important variables in the surfactant enhanced carbon regeneration (SECR) process. The process was found to effectively regenerate the carbon containing either solute at various loading levels. Increasing micellar solution flow rate increased the total volume of solution required to regenerate the bed to a given level, while increasing surfactant concentration in the regenerant solution decreased this solution volume. The removal of toluene was nearly equilibrium limited, while amyl acetate exhibited more significant mass transfer resistance. The residual surfactant in the carbon bed following the regeneration was readily removed by a water flush. The carbon showed no signs of serious degradation over 9 regeneration cycles. A preliminary economic analysis showed that SECR is competitive with steam regeneration, but applicable to a much wider range of volatile solutes. SECR is much less expensive than destructive techniques, such as hearth regeneration, incineration, or land fill, as well as recovering the solute for reuse.

SECR for liquid phase applications was also investigated in which the equilibrium adsorption of cetyl pyridinium chloride (CPC) and sodium dodecyl sulfate (SDS) on activated carbon were measured. CPC showed increased adsorption with increasing temperature. SDS adsorption showed 
no significant temperature dependence. The desorption of CPC from activated carbon in an adsorption bed under dynamic conditions was also studied. The water flush removed approximately 10 to $11 \%$ of the CPC adsorbed on the carbon bed. Breakthrough curves for tert-butylphenol (TBP) indicated a significant loss of adsorptive capacity for the surfactant regenerated bed relative to the virgin carbon bed for a liquid-phase system.

Micellar-enhanced ultrafiltration (MEUF) was investigated using spiral wound membranes for the simultaneous removal of organic compounds, metals and metal complexes dissolved in water, with emphasis on pollution control applications. Removal of $99+$ percent of TBP, and chromate using CPC, a cationic surfactant, was demonstrated experimentally. The removal of $99+$ percent of TBP, copper, and cadmium using SDS, an anionic surfactant, was also demonstrated. The surfactant was successfully recovered for reuse and separated from the organics, metals and metal complexes by precipitation of the surfactant. Economic analysis was performed and showed MEUF and associated precipitation processes are competitive with mature technologies used to accomplish the same end result. The comparison of spiral wound MEUF results with stirred cell MEUF results validated the assumption that the separation is occurring at or near equilibrium.

Investigation of MEUF to remove $99+$ per cent of trichloroethylene (TCE) from contaminated groundwater using criteria such as: membrane flux, solubilization equilibrium constant, surfactant molecular weight, and Krafft temperature led to the selection of an anionic disulfonate with a molecular weight of 642 (DOWFAX 8390). The efficiency of this system was tested in a spiral wound ultrafiltration device and recovery of the surfactant from the concentrated surfactant/TCE solutions using vacuum stripping was 
shown to be feasil $450 \cdots, \quad$ These data and results from supporting experiments were used to design a system which could clean-up water in a 100,000 gallon/day operation. A four stage process was found to be an effective design and estimated cost for such an operation were found to be in the range of the cost of mature competitive technologies. 


\section{CHAPTER 1}

\section{INTRODUCTION}

Western industrialized nations are faced with an escalating percentage of their gross national product being utilized for pollution control on their present production processes as well as significant remedial operations for the clean up of pollution sites that are by-products of past production. Considering that Western industrialized nations are leading the rest of the world in environmental regulation, a significant cost of production advantage is being enjoyed by third world countries that have industries that exist in an environment subject to little or no environmental regulation. The cost advantage held by these third world countries may have a significant effect on the growth rate of the western industrialized nations, thus affecting unemployment and ultimately the standard of living of the developed countries. The potential definitely exists that there is going to be a significant flight of industries with their jobs to third world countries in an effort to evade the restrictive environmental regulations in the developed countries. Examples of this have occurred with U.S. industries that have moved production plants across the border to Mexico which has a minimum of environmental regulations and little enforcement. In order to combat the loss of industries and jobs and a reduction in the standard of living in developed countries, the cost of pollution control and pollution remediation has to be significantly reduced by novel methods. 
The discharge of organics or metals into the environment today as well as past contamination is a serious problem facing developed countries. Some of the most significant pollutants and their sources are:

Trichloroethylene (TCE) is widely used by industry because it is nonflammable, an excellent solvent and at one time was relatively inexpensive, and thus ideal for industrial applications. TCE is used as a solvent for fats, waxes, resins, oils, rubber paints, varnishes, cellulose esters and ethers (1). It is also used for solvent extraction, degreasing and dry cleaning as well as in the manufacture of organic chemicals, and pharmaceuticals (1). TCE has been extensively used for many years, so it is not surprising to find TCE contaminating the groundwater and soil under major industrial operations and waste disposal sites.

The chemical process industries produce wastewater containing numerous and varied dissolved organic components such as chlorinated hydrocarbons or aromatics, which are toxic at low concentrations.

Pesticides and fertilizer can leach from farmland into groundwater supplies. Dry cleaning effluent and solvent from degreasing operations are other sources of organic pollutants.

Dissolved metals, such as nickel, zinc, cadmium, chromate, etc. from the metal plating industries are toxic at extremely low concentrations in wastewater and are highly regulated. Metallic contaminants also are emitted from printed circuit board manufacturer, photographic and photo processing industries, and metal (e.g., uranium) mine tailing leachate. Other metals can appear from feedstocks and catalysts in chemical processing. 
Abandoned metal and coal mines can fill with water, the residual metals can be leached from the rock, and the resultant water enter the underlying aquifer or drain into streams, polluting many communities' groundwater supplies.

Industrial laundries emit wastewater containing both toxic organics (e.g., benzene) and metals (e.g., zinc).

Slaughter houses emit wastewater characterized as having high BOD due to the high levels of fats, oils, and proteins.

The hydrometallurgy processes used by the mining industry produce aqueous waste streams containing dissolved kerosene, ligands, heavy metals and cyanide which are hazardous to the environment.

Refinery or synfuel plant wastewater generally contains both dissolved organics (e.g., phenolics) and dissolved heavy metals (e.g., lead).

Painting and printing industries, dry cleaning industries, refineries and large chemical process plants release large amounts of volatile organic compounds to the atmosphere to the detriment of air quality.

Pollution control and pollution remediation revolves around a separation or a series of separation techniques for removing targeted organic and metal species from water and vapor process streams. The traditional methods that are employed to do these separations includes distillation, ion exchange, adsorption on activated carbon, precipitation, oxidation, and evaporation. Significant amounts of energy are required for distillation, regeneration of activated carbon, and evaporation. The costs of reagents for oxidation, precipitation, and ion exchange are also significant. The problem is exacerbated when the concentration of the target species is 
very dilute to start with. An appropriate example is the precipitation of a very dilute concentration of chromate requires significant amounts of reagent to meet $\mathrm{K}_{\mathrm{sp}}$ requirements. An ever increasing amount of reagent is required as the metal or metal complex becomes more dilute to satisfy the solubility constant. Since ion exchange and activated carbon are not selective, the majority of the bed capacity can be used up by species that do not have to be removed from the process stream thus requiring regeneration of the bed more often at significant expense. New and novel methods that can preconcentrate target species and regenerate activated carbon more efficiently have the potential to reduce capital costs and operating expenses.

Activated carbon is used to remove organics from water or air in numerous applications (2-5). Estimated activated carbon usage in the United States is about 200,000 metric tons/year, making this an extremely important industrial process. Carbon adsorber beds are widely used in gas and wastewater clean-up before emission to the environment and in recovery of organic products from gas or aqueous streams. It is often most efficient to air-strip aqueous streams of volatile toxic organics and use activated carbon to remove the resulting vaporized organics from the gas stream.

While carbon adsorption works efficiently at removal of many organics from vapors, the whole process is limited by regeneration of the carbon. To make the use of granular activated carbon economical, regeneration of the carbon is often necessary (ㄱ). Regeneration of activated carbon is a major factor in the cost effectiveness of the use of carbon (5). 
The standard method of regeneration (3), thermal regeneration, involves removal of the carbon from the bed, transport to a hearth regeneration furnace where the adsorbed organics are volatilized and carbonized at about $1000^{\circ} \mathrm{C}$, and loading the bed with fresh carbon. This process is energy intensive, labor intensive, and time consuming. Further, the organic adsorbate is not recovered, up to $30 \%$ of the carbon may be burned in the furnace, and it is potentially dangerous as the contaminated carbon must be transferred several times. This method is useless if the adsorbed solutes need to be recovered, since they are destroyed in thermal regeneration. An in-situ regeneration method would be a great improvement over this standard regeneration method.

Hot gas regeneration is an in-situ regeneration method in which hot gas (e.g., steam or nitrogen) is passed through the bed to desorb the adsorbate by a combination of purging, and of desorption by heat-up (2,6) . This is only effective when the adsorbate is highly volatile. Solvent regeneration is another in-situ regeneration technique (7-10), in which an organic liquid solvent is passed through the carbon bed to desorb the adsorbate. A major disadvantage of this operation is that when the process is complete, a hot gas regeneration must be performed to desorb the residual volatile solvent, making it an energy intensive process.

In biological regeneration (11), another in-situ regeneration method, bacteria are introduced into the bed to consume the adsorbed organic. Disadvantages include the process being very slow, the organic not being recovered, reduction of bed capacity from adsorption of some of the products of the degradation, the need to induce desorption of the bacteria when done, 
and, finally, the fact that the bacteria often cannot ingest a mixture of organics.

\subsection{Micellar-Enhanced Separation Processes}

Surfactant-based separation techniques are developing into an important class of industrial separation processes $(13,15,24)$. The principal advantages of surfactant-based separation processes are the low energy utilization, and surfactants in general are environmentally harmless and considered to have a low toxicity (13,15).

Micellar-enhanced ultrafiltration (MEUF) and surfactant enhanced carbon regeneration (SECR) are two novel separation techniques which are based on the ability of surfactant micelles to solubilize organic compounds and electrostatically attract ionized metals and metal complexes in aqueous solutions as shown in Figure I.1.

MEUF provides the opportunity to remove both dissolved organics and metals simultaneously from wastewater in a process which is potentially more efficient than alternative methods. In fact, almost all other methods either remove only organics or metals. MEUF also has the ability to remove either organics or metals individually in an efficient process.

An illustration of MEUF applied to the removal of an organic and a multivalent cationic metal from water is shown in Figure I.2. An anionic surfactant is added to the water at concentrations well above the critical micelle concentration. Under this condition, the vast majority of the 
surfactant is present as micelles. Micelles are surfactant aggregates containing 50-150 surfactant monomers (16). The interior of the micelle is a hydrocarbon-like environment. Dissolved organic solutes originally in the water tend to dissolve in the interior of the micelles or "solubilize" in the micelles. Since anionic surfactants are used, micelles have a very high negative charge. Any multivalent cations in solution bind or adsorb onto the opposite charged micelle surface. Therefore, heavy or transition metals which are multivalent cations (e.g., $\mathrm{Cu}^{2+}, \mathrm{Pb}^{2+}, \mathrm{Al}^{3+}, \mathrm{Cd}^{2+}$ ) are attached to micelles by electrostatic attraction (17,18). If a cationic surfactant is used instead of an anionic surfactant, the micelle can bind multivalent anionic metal complexes (e.g., chromate or $\mathrm{CrO}_{4}^{2-}$ ) (19). The strisam containing the products or pollutants may be reduced even further in volume during the surfactant recovery/recycle operation.

The solution is then treated by ultrafiltration. The filter membrane pore sizes are small enough to block the micelles with the organic solute and metal species associated with these micelles. The concentration of the organic, metal species and surfactant in the permeate (solution passing through the membrane) correspond to their unassociated concentration in the solution which does not pass through the membrane (retentate). For a properly designed system, the permeate concentrations of the target organics and metal species can be very low. The permeate can be discharged to the environment or reused in the plant. The retentate contains almost all of the original solutes in high concentration and is much smaller in volume than the original stream treated $(15,18,20)$. Therefore MEUF can achieve waste minimization or preconcentration of the targeted 
organic compounds by concentrating pollutants or products inte a stream of relatively small volume in one pass.

MEUF was initially envisioned as an analytical method to investigate micellar phenomena. Researchers have analyzed permeate solutions in an effort to investigate monomer-micelle equilibrium in the retentate solution (12.23.24), counter ion binding to the micelle surface (17.23), and the solubilization of organic solutes by the micelle (12,21,22,25,26). In several of these investigations, very low permeate flux rates were used to enhance equilibrium between the retentate and permeate solutions. Partial rejection of the monomer has been observed for ultrafiltration membranes with very small pore diameters (12.21.24). The essence of these studies is that MEUF can concentrate solubilized organics and electrostatically bound multivalent counterions effectively in the laboratory setting. The significance of these studies is that MEUF offers the potential of alternate industrial separation techniques that may offer reduced capital and operating costs when compared to traditional separation techniques. The work presented in this dissertation shows the technical viability of scaling up the MEUF process from the laboratory to industrial scale using off-the-shelf commercially available ultrafiltration membranes.

SECR uses surfactants (detergents) to remove adsorbed organics from activated carbon in order to regenerate it for reuse (27). In SECR, a concentrated surfactant solution is passed through the spent carbon bed. The adsorbate desorbs and is solubilized into micelles in the solution as shown in Figure I.3. A concentrated surfactant solution can contain large 
concentrations of dissolved organics through solubilization. Therefore a small volume of the concentrate surfactant solution can potentially solubilize all of the adsorbed organic resulting in a small stream which is highly concentrated in the organic adsorbate or solute, SECR is composed of three steps as shown in Figure 1.4. Step one is the separation of the target organic from the process stream. Step two is the countercurrent flow of regenerant surfactant solution to "solubilize" the organic compounds adsorbed on the activated carbon surface. Step three is a water flush to remove residual surfactant form the activated carbon bed. A fourth step may be added to air or nitrogen dry the carbon bed for vapor applications. At this point the bed is regenerated and ready to begin at step one again.

MEUF and SECR have the potential of significantly reducing the volume of wastewater process streams at ambient temperatures and at relatively moderate (60 psi.) or ambient pressures. However, the resultant concentrated surfactant solution with solubilized organics, metals and metal complexes needs further processing in order to recycle and reuse the surfactant. Traditional separation processes such as vacuum or steam stripping, precipitation, or liquid/iquid extraction can now be applied with the potential of a significant reduction in plant capital cost and operating costs because the waste stream resulting from MEUF or SECR is very small and concentrated relative to the original waste stream. 


\subsection{Scope of Work}

The studies presented in this work are predominantly phenomenological in nature. The main thrust or goal of this work is to evaluate SECR and MEUF for industrial/commercial application and to address fundamental problems associated with scale-up of the processes. The studies are varied and diverse, interlacing many subjects. Therefore, Chapter 2 gives an overview of background information on the subjects relevant to comprehension of the material covered in this dissertation. Experimental techniques and procedures are briefly described in Chapter 3. The experimental work is organized into five basic subdivisions. The first study, Chapter 4, investigates the use of SECR for vapor phase activated carbon adsorber systems. The second study, Chapter 5 investigates the use of SECR for liquid phase activated carbon adsorber systems. The third study, Chapter 6, primarily compares available laboratory stirred cell MEUF and semiequilibrium dialysis data to pilot plant spiral wound MEUF data for the rejection of target organics, target metals, surfactants, and relative flux rates. The final study, Chapter 7 , investigates the recovery of chlorinated volatile organic compounds (VOCs) from an aqueous stream and the preliminary design of a process which includes a surfactant recycle step and the isolation of the target VOCs into a small organic stream. 


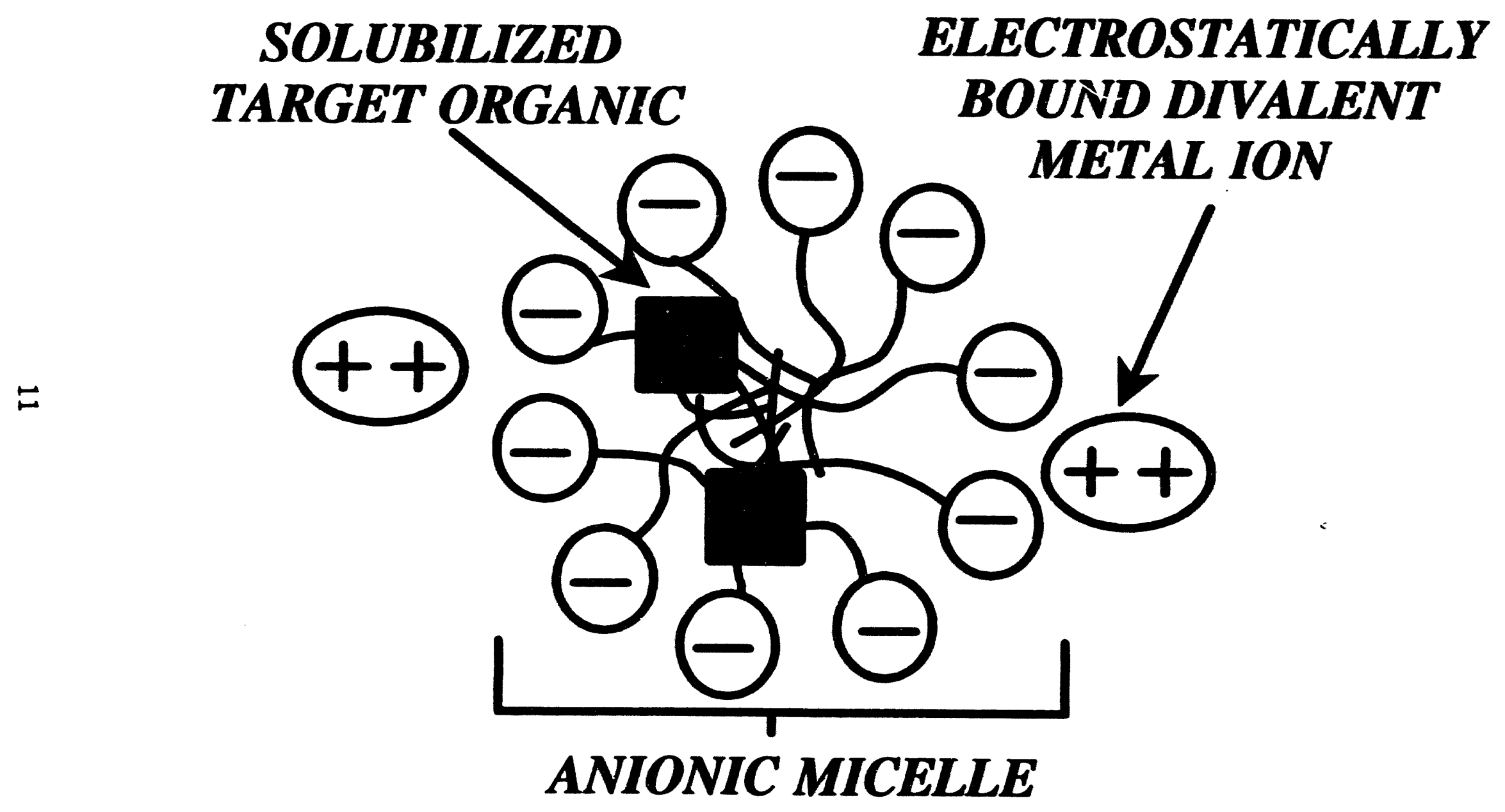

FIGURE I.1 Illustration of a Target Organic Solubilized in the Interior of a Micelle and a Divalent Metal Ion Electrostatically Bound to the Surface of a Micelle 


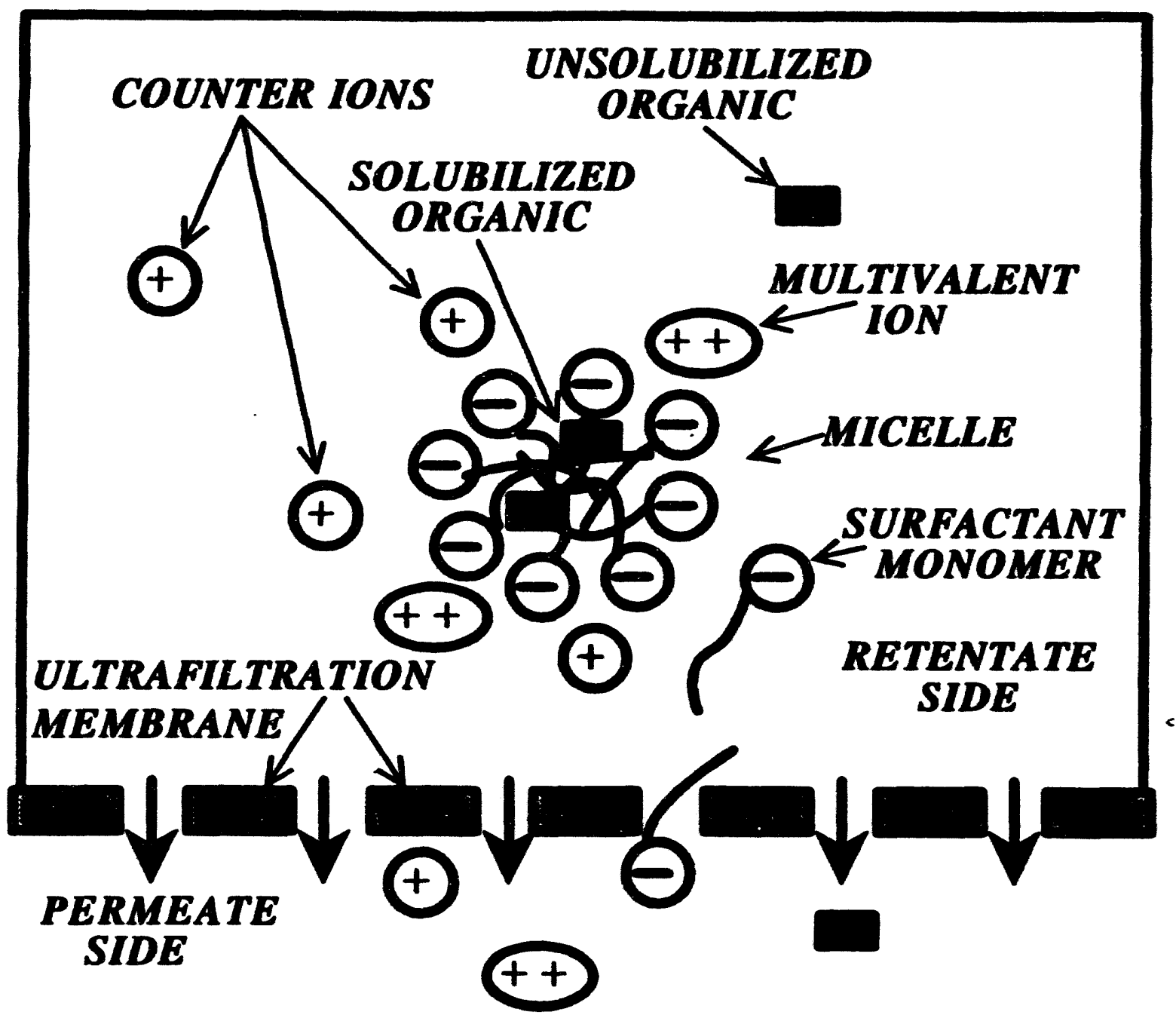

FIGURE I.2 Illustration of MEUF Applied to the Removal of a Target Organic and a Target Multivalent Cationic Metal in an Aqueous Solution 


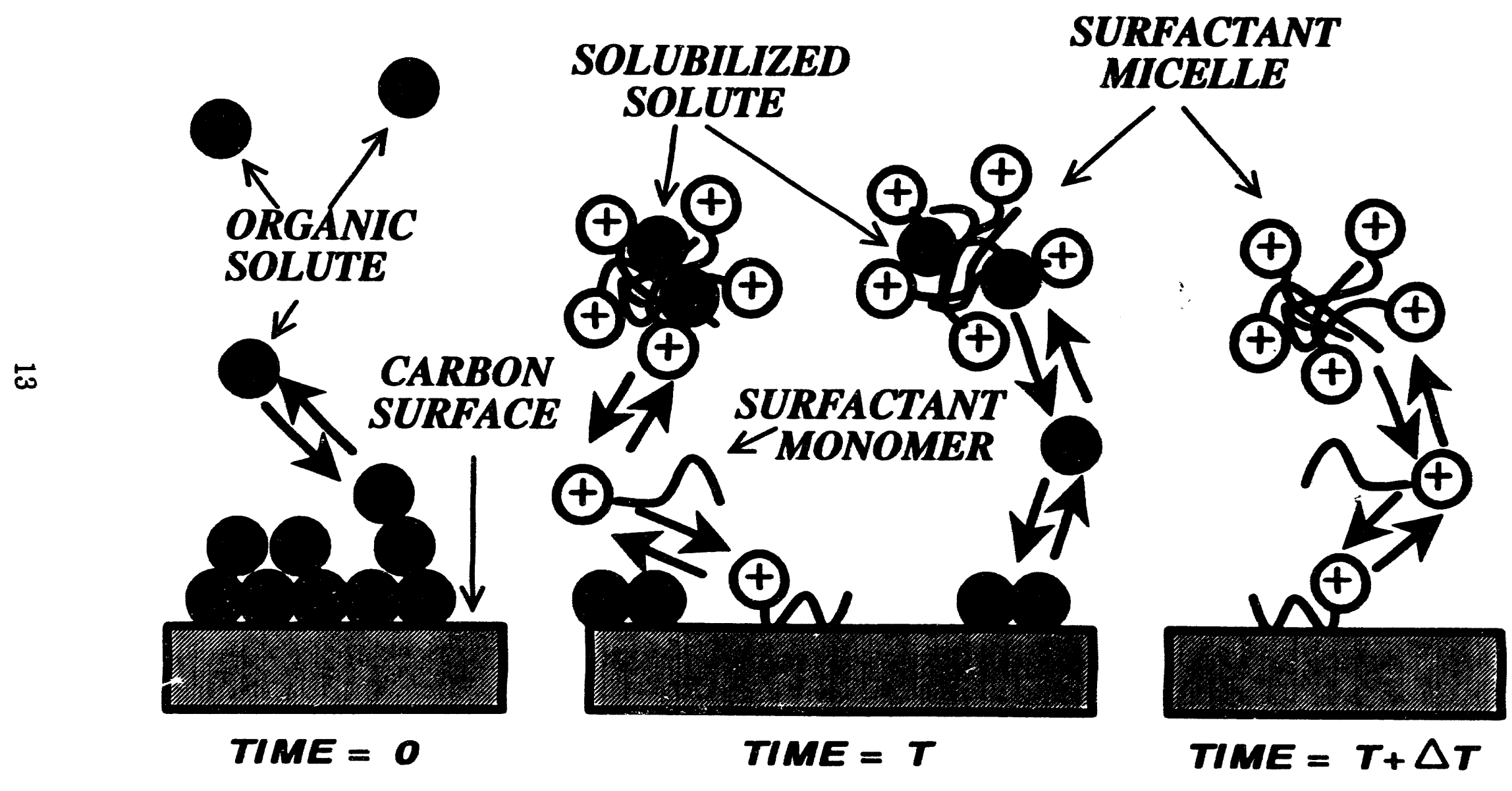

FIGURE I.3 Illustration of the SECR Concept 


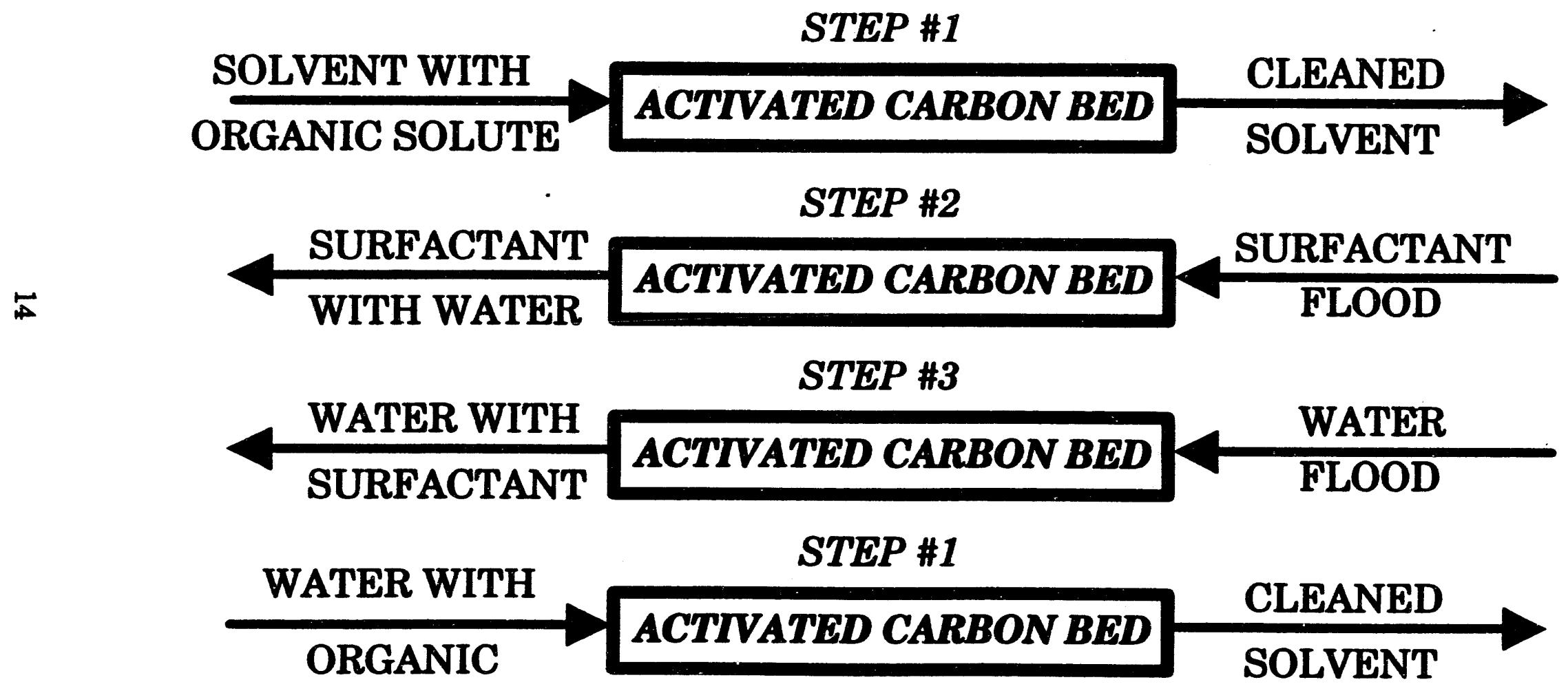

FIGURE I.4 Illustration of the Three Basic Steps Required for the Application of SECR 


\section{CHAPTER 2}

\section{BACKGROUND}

Taking micellar-enhanced separation techniques from the laboratory to the industrial setting requires not only an understanding of the basic concepts related to the separation process but also a consideration of available process industry equipment and how it is utilized and operated. This chapter reviews the following basic concepts :
a. ultrafiltration
b. micelle formation by surfactants
c. solubilization of dissolved organic molecules
d. adsorption of multivalent counterions onto micelles
e. semiequilibrium dialysis
f. stirred cell apparatus
g. spiral wound apparatus
h. adsorption of surfactant at the solid-liquid interface
i. characteristics of carbon beds

The concepts listed above set the foundation for the research presented in Chapters 4-7 and the conclusions presented in Chapter 8.

\subsection{Ultrafiltration}

Ultrafiltration (UF) technology is only about 30 years old but has found a wide variety of applications such as electrocoat paint recovery, latex processing, recovery of lubricating oils, reduction of machining coolant 
wastes, breaking of emulsions, textile size recovery and kidney dialysis (28). UF is also finding applications in conjunction with bioreactors, harvesting of microbial cells and fractionation of macromolecules (28). The market for UF in 1986 is estimated to have been 400 to 600 million dollars (28). This indicates that UF technology has developed to a point of industrial acceptance and utilization. There are many companies that manufacture and market UF membranes and UF systems. These products are considered off-the-shelf purchases that are easily integrated into many applications. UF is one of many membrane separation techniques as shown in Figure II.1. Three of them, microfiltration (MF), reverse osmosis (RO), and ultrafiltration, depend on pressure as the driving force $(29,30)$ as shown in Figure II.2. Figure II.3 relates the relative size of some common materials and approximate molecular weights to the range of application for pressure driven membrane separation processes. In general UF membranes are effective for molecular weights from 500 to 300,000 .

\subsubsection{Ultrafiltration Membranes}

Sourirajan and Loeb made a breakthrough contribution in 1960 with the development of the asymmetric membrane (28). The asymmetric membrane consists of a thin film of polymer adhering to a porous backing material. The separation occurs at the interface of the liquid phase and the thin polymer film. The backing material provides the mechanical support for the thin polymer film. The asymmetric membrane has higher flux rates 
compared to a symmetric membrane by minimizing the thickness of the polymer film and thus reducing the mass transfer resistance..

The molecular weight cutoff (MWCO) of a membrane is characterized empirically by the rejection of a spectrum of water soluble molecules ranging form zero to $100 \%$ rejection. Sodium chloride (MW 58.5) and glucose (MW 180) are two examples of low molecular weight compounds that are anticipated to have zero percent rejection. Immunoglobins, large proteins, (MW >900,000) and blue dextrin are examples of high molecular weight compounds that should be completely rejected. The characterization of the rejection profile is accomplished by selecting a series of solutes between the range of the low and high molecular weight compounds such as those listed above and testing their rejection using a UF membrane. In general, a specified MWCO for a specific UF membrane corresponds to $90 \%$ of the compounds of that molecular wei ht being rejected. Of course, compounds with molecular weights higher than the MWCO of the membrane should have rejection rates higher than $90 \%$. There is some evidence that flux rates increase as molecular weight cut off increases (22) however this is dependent on a uniform number of pores per unit area from one molecular weight cut off to another. In essence, the porosity of the membrane depends on both the size of the pores and the number of pores per unit area.

The rejection of a solute is described by:

$$
\mathbf{R}_{\mathbf{b}}=\mathbf{1} \cdot\left(\mathbf{C}_{\mathbf{p}} / \mathbf{C}_{\mathbf{b}}\right)
$$


where $R_{b}$ is the rejection of the solute based on the bulk retentate concentration, $C_{p}$ is the concentration of the solute in the permeate, and $\mathrm{C}_{\mathrm{b}}$ is the concentration of the solute in the retentate.

\subsubsection{Concentration Polarization}

Ultrafiltration is a barrier separation technique. The basis for the separation is that the pores of the barrier are too small for the solute to pass through the barrier. Thus, there is an elevated concentration of rejected solute near the surface of the membrane. Laboratory apparatus, such as stirred cells, and industrial apparatus, such as spiral wound and hollow fiber systems, use a cross flow method to minimize the elevated solute concentration near the membrane. A cross flow pattern is the flow of the bulk solution on the retentate side of the membrane parallel to the membrane surface as shown in Figure II.4. This "sweeping" action across the surface of the membrane minimizes the elevated solute concentration near the retentate side of the membrane $(29,30)$. However, as with any flow parallel to a surface, there is a hydrodynamic boundary layer characterized as a laminar flow region where mixing is not present. Within this region, the concentration of the rejected solute is higher than the bulk solution and the phenomenon is referred to as concentration polarization (28,31). Concentration polarization (CP) can have a significant adverse affect on the flux rate of the solvent through the barrier. An analogous example is the build up of a "cake" in particle filtration and the subsequent significant reduction in the flux of the solvent through the barrier. In 
essence there are two resistances to the flow of solvent to the permeate side of the membrane as illustrated by (30):

$$
\mathbf{J}_{\mathbf{w}}=\Delta \mathbf{P} /\left(\mathbf{R}_{\mathbf{c}}+\mathbf{R}_{\mathbf{m}}^{v}\right)
$$

where $J_{W}$ is the solvent flux through the membrane, $\Delta P$ is the applied pressure drop, and $R_{c}$ is the resistance to flux through the laminar region, and $R_{m}$ is the resistance to the flux through the membrane,. Therefore, $R_{c}$ is analogous to a "cake' resistance in particle filtration and is a function of the solute concentration in the laminar region. As the solute concentration increases, $R_{c}$ increases and can have more of an affect on the solvent flux rate than $R_{m}$ at elevated bulk retentate solute concentrations where concentration polarization is well developed.

Concentration polarization not only affects solvent flux through the membrane but may also affect the rejection of the solute. Rejection is based on the bulk concentration of the solute in equation 2.1. In reality, a more accurate representation of rejection needs to be based on the elevated concentration of the solute at the membrane surface since the bulk concentration may be substantially lower than the concentration of the solute at the membrane surface. Therefore, in equation 2.1 the substitution of bulk concentration, $\mathrm{C}_{\mathrm{b}}$, by the concentration of the solute at the membrane surface, $C_{s}$, provides a description of the true rejection, $R_{t},(30)$ :

$$
R_{t}=1 \cdot\left(C_{p} / C_{s}\right)
$$


The concentration of the solute at the membrane surface is not easily measured. Also, depending upon how the ultrafiltration process is operated, the $\mathrm{C}_{8}$ may not be constant but increasing with time in the event of a batch process with the constant removal of the permeate as in a stirred cell apparatus. Therefore, the rejection based on the bulk concentration of the solute using equation 2.1 is generally used.

\subsubsection{Gel Polarization}

The reduction of flux caused by concentration polarization for pressure-driven membrane separation techniques is generally explained by one of two different mechanisms, gel polarization or osmotic pressure. The elevated concentration of solute near the membrane surface causes an increase in the osmotic pressure at the interface of the retentate. Since the driving force pressure is equal to the total transmembrane pressure minus the osmotic pressure, increased osmotic pressure causes a reduction in flux. Reverse osmosis is generally more severely affected by the osmotic pressure than ultrafiltration (30).

The alternate view is that the formation of a densely packed region of rejected solutes at the retentate solution/membrane interface within the boundary layer causes increased rejection and decreased permeate flux due to increased resistance to flow. This is referred to as gel polarization. Shown in Figure II.4 is an illustration of the concept of concentration polarization with gel polarization of macromolecules and colloids during the UF process. As the solute bulk concentration increases, the permeate flux 
decreases until the solute bulk concentration approaches the gel concentration and the permeate flux approaches zero.

The basis for the concept is that the back diffusion of rejected solutes from the membrane surface into the bulk solution is in balance with solutes carried to the membrane surface by convective flow. As the solute bulk concentration $\left(\mathrm{C}_{\mathrm{b}}\right)$ increases, the driving force for the diffusion of rejected solutes in the laminar sub-layer decreases. The driving force for solute diffusion is the solute conce:atration difference between the solute bulk concentration and the solute gel concentration, $\mathrm{C}_{\mathrm{g}}$, as illustrated by Fick's rate equation for a homogeneous phase:

$$
J_{\mathbf{a}, z}=\cdot \mathbf{D}_{\mathbf{a}, \mathbf{b}}\left(d \mathbf{c}_{\mathbf{a}} / d z\right)
$$

where $\mathrm{J}_{\mathrm{a}, z}$ is the molar flux of solute $\mathrm{a}$ in the $z$ direction, $d \mathrm{c}_{\mathrm{a}} / d z$ is the concentration gradient in the $z$ direction, and $D_{a, b}$ is the diffusion coefficient of solute $a$ in solvent $b$ (32). A generalized form of equation (2.4) is (33):

\section{FLUX = - (diffusion coefficient) (concentration gradient)}

It becomes obvious that as the bulk solute concentration approaches the solute gel concentration, the solute concentration gradient decreases, thus decreasing the diffusion of solute back to the bulk solution. When the solute gel concentration is equal to the solute bulk concentration, there is 
no concentration gradient and thus no diffusion of solute from the gel layer to the bulk solution. Since it is difficult with the technology available to evaluate and quantify the thickness of the gel layer or the concentration gradient of the solule in the boundary layer, equation 2.4 is of little practical use in this application but does serve to help illustrate the concepts of gel polarization and concentration polarization.

A relationship with practical application based upon the steady state assumption described in gel polarization mechanism is (21):

$$
J_{W}=K\left[\ln \left(C_{g} / C_{b}\right)\right]
$$

where $J_{W}$ is the flux rate of the solvent through the membrane, $K$ is a mass transfer coefficient, $C_{g}$ is the solute gel concentration and $C_{b}$ is the solute bulk concentration. When $\mathrm{C}_{\mathrm{g}}$ and $\mathrm{C}_{\mathrm{b}}$ are equal, the solvent flux through the membrane is zero. This is based on the assumption that there is no diffusion of the solute from the gel layer to the bulk solution and therefore, there can be no convective transfer of solute to the gel layer implying that there is no convective flow of solvent to the gel layer or membrane surface and therefore no mass transfer of solvent through the membrane. A semilog plot of flux verses bulk solute concentration results in a straight line with a slope of $K(21,29,30)$. The mass transfer coefficient from the plot is a mass transfer coefficient for the membrane in conjunction with the concentration polarization characteristic of that particular membrane and solute/solvent system. Therefore, the application of equation 2.5 requires a 
data base of values obtained by experimentation for the specific membrane and solute/solvent system to be described.

The gel layer is reversible and can be manipulated. The gel layer can be eliminated by setting the transmembrane pressure to zero and allowing the solute concentration gradient to dissipate. However, it will reform almost immediately when the transmembrane pressure is applied. During operation, the gel layer can be minimized by increased cross flow over the surface of the membrane, minimizing the boundary layer, thus increasing the solute concentration gradient and thus increasing the flux of the solute from the gel layer to the bulk solution. This minimizes the gel layer thickness and increases the solvent flux through the membrane.

Osmotic pressure has been shown to be significant in the ultrafiltration of dextran and other large macromolecules (34). The formation of a gel layer has been shown to be the major mechanism for reduced flux for UF of colloids, such as those used in MEUF (34-36). Others have theorized that both osmotic pressure and gel layer formation simultaneously affect UF of colloidal suspensions (34). Recent work using surfactants supports the assumption that the gel layer mechanism is the primary mechanism affecting MEUF (21).

\subsubsection{Stirred Cell}

The stirred cell, the primary ultrafiltration apparatus used for this study of MEUF, is significantly different from the ultrafiltration systems used in industrial processes. Shown in Figure II.5 is a schematic of a 
stirred cell illustrating its main components and general geometric shape. The transmembrane pressure is provided by compressed nitrogen at $60 \mathrm{psi}$ on top of a liquid solution within the acrylic tube. The acrylic tube is sealed by O-rings in nylon end caps. The cross flow of the solution is provided by a magnetic stirrer bar mounted approximately 2 millimeters above the UF membrane which sits on a porous support base. The retentate solution is contained within the acrylic tube while the permeate exits the stirred cell apparatus through the bottom nylon end cap. The entire pressure relief valve subsystem unscrews off the top end cap to allow charging of the stirred cell with a solution.

The operational characteristics of the stirred cell are shown in Figure II.6. In general the stirred cell is operated in a batch mode. Any portion of the initial charge of solution in the cell is either retained in the cell or flows through the membrane as permeate. The volume of the solution in the cell decreases during the experiment while the concentratioia of the rejected solute in the retentate increases as is illustrated by the curve to the right of the cell in Figure II.6. The permeate is constantly being withdrawn, resulting in a constantly increasing rejected solute concentration in the retentate. Therefore, concentration polarization, gel polarization and flux through the membrane are continuously changing and may not achieve equilibrium conditions. Also shown in Figure II.6 is a characteristic velocity profile based on the linear velocity of the magnetic stirrer bar. The velocity is at a minimum at the very center of the magnotic stirrer bar and reaches a maximum at the ends of the magnetic stirrer bar. This suggests that the flux and rejection change as a function of radius since linear 
velocity of the solution has a significant affect on boundary layer thickness and therefore, gel and concentration polarization characteristics. The radial flow and constantly changing concentration characteristics of the stirred cell differ from the flow and concentration characteristics of industrial membrane systems.

\subsubsection{Spiral Wound Membrane System}

The spiral wound membrane, shown in Figure II.7, is a typical membrane configuration used widely in industry today. The spiral wound membrane is composed of two sheets of asymmetric membrane sandwiching a screen spacer and sealed on three sides. It is helpful to think of this as a mailing envelope with one of the short lengths of the envelope cut open to remove the letter (screen spacer) inside. The open end of the envelope is cemented into a PVC tube so that whatever permeates to the interior of the envelope flows into the interior of the PVC tube and is removed from the membrane unit. In order to make this more compact, the envelope is wound around the PVC tube along with another spacer. The membrane is held in a pressure vessel that allows the feed to flow in a channel over the outside of the envelope. The screen in this channel provides a significant amount of turbulence, thus providing good mixing and reducing the boundary layer and the resulting concentration polarization. The primary advantage of the spiral wound configuration is the large membrane surface area available in a very compact size. 
Figure II.8 illustrates some of the operational characteristics of a spiral wound membrane. The concentration profile shows a gradual increase in the retentate concentration with the minimum occurring at the feed end of the module and the maximum retentate concentration occurring at the retentate outlet of the module. The concentration at any point in the spiral wound UF unit is invariant with time unlike the concentration above a membrane in a stirred cell apparatus. The velocity profile illustrated by Figure II.8 shows a gradual change in velocity with the maximum at the feed inlet of the module decreasing to a minimum at the retentate outlet of the module. The velocity profile remains constant with a relatively small velocity change when compared to the velocity profile of the solution flowing over a membrane in a stirred cell.

\subsection{Surfactants}

A surfactant is a schizophrenic molecule composed of two distinct moieties, one being hydrophobic and the other hydroph ic. Shown in Figure II.9 is a schematic of a surfactant monomer illustrating an anionic surfactant, sodium decyl sulfate (SDS), and the associated hydrophobic and hydrophilic regions. The hydrophobic region is generally characterized as a hydrocarbon or fluorocarbon chain that is relatively insoluble in water such as the $\mathrm{C}_{10} \mathrm{H}_{21}$ alkyl chain of SDS illustrated in Figure II.9. The hydrophilic region is relatively water soluble and may be classified into two major groups, ionic and nonionic, depending on the characteristics of 
the hydrophilic region. The hydrophilic region, often referred to as the "head group" or "head" is ionic if it is charged while a nonionic surfactant may have a polyethoxylate group as a head. The hydrophilic group for SDS in Figure II.9 is the $\mathrm{SO}_{4}{ }^{-}$group, since the sodium ion dissociates in a polar solvent upon dissolution of the surfactant.

\subsubsection{Micelle Formation and Structure}

Surfactant molecules exist, in solution, as individual and unassociated monomers below the critical micelle concentration (CMC) or as a combination of surfactant monomers in equilibrium with surfactant aggregates known as micelles. The critical micelle concentration (CMC) is the minimum surfactant concentration at which surfactant monomers form micelles (37.38,16). Shown in Figure II.10 is an illustration of the relationship between total surfactant concentration, monomer concentration and CMC. The total surfactant concentration and monomer concentration are equal until the total surfactant concentration reaches the CMC at which point the monomer concentration remains approximately constant. Above the CMC, any additional surfactant added to the solution forms aggregates called micelles. Shown in Figure II.11 is an illustration of a spherical micelle composed of anionic surfactant in an aqueous solution. The hydrophobic tails are oriented toward the center of the micelle and the (anionic) head groups are oriented outward in contact with the polar solvent. The interior of the micelle is a hydrophobic core of intertwined tail groups into which other hydrophobic constituents may solubilize to escape a 
polar solvent environment. The outer surface of the micelle may have a high charge density with ionic surfactants. Multivalent ions of opposite charge to that of the ionic surfactants tend to adsorb or bind on the micelle surface due to electrostatic attraction. Micellar aggregate geometries other than the spherical structure are possible such as rod and laminar shapes.

\subsubsection{Models Describing Micelle Formation}

There are two predominant models for describing monomer-micelle equilibrium, the pseudo-phase separation model and the mass action model (37,38). The pseudo-phase separation model describes monomer-micelle equilibrium based on a thermodynamic analogy to liquid/vapor equilibrium (38). In this analogy the micelle is treated like a condensed phase (liquid) while the monomer is analogous to the vapor phase. The essence of this model is the assumption of a constant monomer concentration at total surfactant concentrations at or above the critical micelle concentration. This is analogous to a single component liquid/vapor system at a constant temperature where the pressure of a closed container is constant (equal to the vapor pressure) even if additional moles of the compound are added to the system. The vapor pressure at the dew point is analogous to the monomer concentration at the CMC.

The mass-action model describes monomer-micelle equilibrium as a chemical equilibrium :

$$
\mathbf{n A} \Leftrightarrow \mathbf{A}_{\mathbf{n}}
$$


where $n$ is the number of surfactant molecules in a micelle and is derignated the aggregation number. The equilibrium constant, $\mathrm{K}$, is described by:

$$
K=\left[\mathbf{A}_{\mathbf{n}}\right] /[\mathbf{A}]^{\mathbf{n}}
$$

Unlike the pseudo-phase separation model, the mass-action model considers the presence of some micelles below the CMC. The two models agree fairly well when the micelle aggregation number is larger than 50 (38).

\subsection{Solubilization of Dissolved Organic Molecules}

Micelles have an interior that is characterized as nonaqueous and nonpolar. It is this hydrophobic environment that solubilizes organic solutes that are dissolved in the aqueous solvent (39-41). The nonpolar nature of the environment in the interior of a micelle changes as a function of radius. The center of a micelle exhibits the maximum nonpolar characteristics of any region in the micelle while this nonpolar environment diminishes to a minimum at the perimeter of the micelle in the region of the polar head group. The micelle is divided into locations into which organic solutes may solubilize: $(40,42,43)$.

a. the inner hydrocarbon core, the most non polar region

b. the palisade layer 
c. at the micelle-solvent interface

d. between nonionic surfactant headgroups such as polyoxyethylene chains.

In essence, organic solutes have a wide spectrum of regions of varying degrees of nonpolarity into which they may solubilize.

Organic solutes solubilize into those regions of the micelle which have similar nonpolar characteristics to those of the organic solute. Shown in Figure II.12 is an illustration of an anionic micelle and the location of at which solubilization of different types of organic solutes may occur. Nonpolarizable organic solutes such as saturated aliphatic hydrocarbons are solubilized in the interior region of the micelle $(40,42)$. Long chain alcohols, upon solubilization in the micelle, may orientate with the polar hydroxyl group near the surfactant headgroups in the palisade layer and the hydrocarbon chain oriented toward the interior of micelle with some hydrogen bonding between the tails of the surfactants and hydrocarbon chain of alcohol (43). There is some evidence that short-chain phenols and other polarizable organic solutes solubilize between the polyoxyethylene chains of nonionic surfactants (43). Shown in Figure II.13 is an illustration of the solubilization of polar organic solutes in the hydrophilic polyoxyethylene head groups.

\subsubsection{Solubilization Constant}

In micellar solutions, organic solutes are distributed between the solubilized and unsolubilized states. This distribution may be described by (37): 


\section{$\mathbf{K}=\mathbf{C}_{\mathbf{s}} / \mathbf{C}_{\mathbf{m}} \mathbf{C}_{\mathbf{u}}$}

where $K$ represents the distribution constant, $C_{s}$ is the concentration of the organic solute that is solubilized, $\mathrm{C}_{\mathbf{m}}$ is the total surfactant concentration minus the CMC of the of the system to yield the concentration of surfactant monomer in the micellar from. The unsolubilized organic solute concentration is $\mathrm{C}_{\mathrm{u}}$. Henry's law is often assumed to apply to the solubilization equilibrium. This is interpreted to mean $\mathrm{K}$ is independent of concentration. $\mathrm{K}$ increases as the fraction of organic solute solubilized increases. Generally, the more hydrophobic or non polar the organic solute, the greater the tendency to solubilize into the micelle and the higher the value of $K$.

Several different techniques are used to study the distribution of organic solutes between the micelle and the solvent. The maximum additivity method $(\underline{40,42})$ has been used by many studies and is shown in Figure II.14. This requires the organic solute to be immiscible with the aqueous phase as shown Figure II.14. In essence, the aqueous phase is saturated with the organic solute defining the maximum level of organic solute in the micelle. After equilibration, the aqueous solution is analyzed and the concentration of the organic solute in the aqueous surfactant solution is determined. The amount of organic solute in the micelle is the difference between the total concentration of the organic solute in the aqueous phase and the solubility of the organic solute in pure water. Henry's law is sometimes assumed to apply to predict solubilizations for 
organic solute concentration less than saturation (40). Using Henry's law in this manner results in solubilization distribution constants that do not effectively describe experimental data for many systems $(42,44,45)$.

Two other techniques, head space analysis and vapor pressure analysis, rely on vapor pressure to evaluate the distribution of an organic solute between the micelle and solvent over a significantly larger range than the maximum additivity method $(22,43,45)$. The major requirement for these techniques is that the organic solute is volatile enough that the vapor pressure above the aqueous phase is measurable or concentrated enough for analysis using gas chromatography. These techniques yield extremely good data for volatile organic solutes.

Semi-equilibrium dialysis (SED) is a versatile technique for studying the distribution of an organic solute between the micellar phase and the solvent. SED is a membrane separation technique that relies on a concentration difference as the driving force for the separation, unlike ultrafiltration which relies on pressure as the driving force for separation. Figure II.2 shows that dialysis and ultrafiltration have similar definitions for permeate and retentate. The permeate for both processes is composed of water and small molecules which can pass through a memebrane, while the retentate for both processes is composed of large molecules or aggregates of molecules. These similarities allow the use of SED equipment to measure solubilization and counterion binding information comparable to stirred cell ultrafiltration data. The simplicity of SED equipment and the ease of the experimental technique allow the accumulation of data which can be used to estimate separation efficiency more conveniently than by ultrafiltration 
experiments. Shown in Figure II.15 is an illustration of a semiequilibrium dialysis cell. The cell is composed of two blocks of acrylic with concave chambers. The driving force for the separation is the thermodynamic activity difference across the membrane that divides the chambers in an assembled cell. The membrane is generally isometric instead of the asymmetric type membranes used in ultrafiltration. The chambers are filled through the drilled and tapped holes in each block. One chamber of the cell is filled with the micellar surfactant solution with the dissolved and solubilized organic solute (retentate side). Distilled and deionized water fills the other chamber on the other side of the membrane (permeate side). The cells are allowed to equilibrate in a temperature controlled bath for 24 hours (44) at which time samples are removed from the chamber on both sides of the membrane. The solutes reach equilibrium within 24 hours. The surfactant continues to diffuse slowly from the retentate to the permeate due to a higher activity for the surfactant on the retentate side of the membrane when compare to the permeate side. This results in a slow buildup of micelles in the permeate side with solubilized solute, requiring a amall correction factor in analysis of the data. The surfactant and organic solute concentrations are determined analytically to establish the distribution of organic solute between the micellar phase and the solvent. Since there is no convective mass transfer of solution from the retentate to the permeate during the semiequilibrium dialysis process, concentration polarization or the formation of a gel layer is not present as there are in the ultrafiltration process. 
A relatively new technique for evaluating the partition of organic solutes between the micellar phase and the solvent is micellar mobile phase chromatography (46-50). The technique generally uses standard high performance ïquid chromatography equipment with a reverse phase stationary phase and a mobile phase of surfactant above the critical micelle concentration. Figure II.16 shows the solute equilibrium that is established between the bulk solution, stationary phase and the micellar phase. The residence time of the solute in the column is dependent on the interaction or partitioning of the solute between the various phases where: $\mathrm{K}_{\mathrm{gw}}, \mathrm{K}_{\mathrm{sm}}$, and $\mathrm{K}_{\mathrm{mw}}$ are the partition coefficients for the solute between the various phases. The partition coefficients are obtained by mathematical manipulation of known and measured values. The technique yields excellent results and is easily applied.

\subsection{Phase Change}

Phase change is the basis for many separation processes. However, an unexpected phase change of a component in a process stream may be disastrous or at the least costly. Two of the most obvious and perhaps important parameters related to phase change of surfactants are the Krafft temperature for ionic surfactants and the cloud point for nonionic surfactants. The Krafft temperature for an ionic surfactant in an aqueous solution is the temperature below which an ionic surfactant precipitates. The Krafft temperature is also a function of counterion concentration as well as the type of counterion (e.g. monovalent or multivalent). The cloud 
point for a nonionic surfactant in water is the temperature above which phase separation occurs with a water-rich phase and a surfactant-rich phase coexisting. The water-rich phase has surfactant concentrates up to about 10 times the CMC (51) while the surfactant-rich piase can be thick and viscous and have several tenths weight fraction surfactant.(51). Both precipitation and phase separation can negatively impact an MEUF process by reducing the number of micelles for solubilization and counterion binding as well as potentially fouling the membrane.

Other physical limitations are related to the concentration of the surfactant. At elevated concentrations, ionic as well as nonionic surfactants can form liquid crystals and nonionic surfactants may form a separate phase. These phase changes are also undesirable for an MEUF process.

In general, the type and concentration of the solute can significantly affect the temperature and concentration at which a surfactant may undergo a phase transition. For an industrial application, it is desirable to have as much information about the solutes in a feed stream as well as the feed stream temperature so a screening process can identify likely successful candidates.

\subsection{Courterion-Micelle Interaction}

Shown in Figure II.17 is an illustration of an anionic micelle with the charged head groups at the outer boundary of the micelle. However, this is only a simple two-dimensional perspective of a three dimensional 
roughly spherical shape composed of generally $50-150$ monomers. The result of this dense head group packing along the boundary of a sphere is a charge density and absolute electrical potential that are very high at what is considered the micellar surface (52). It is important to emphasize that not all micelles are spherical. They may exist as rods and sheets but the aforementioned charge density phenomena still applies due to dense packing of the ionized surfactant head groups. The above characterization is also valid for cationic surfactant micelles.

Due to the high charge density at the micelle surface, oppositely charged ions (counterions) are electrostatically attracted to the micelle (17). A distribution of the counterion concentration around the micelle is shown in Figure II.18. The concentration of the counterion initially remains constant and then decreases with increasing distance away from the micelle. The elevated concentration of counterions surrounding a micelle are a surface excess existing within two regions depending upon the nature of the counterion concentration, electrical potential and distance from the micelle surface. The Stern layer is characterized as a region where counterions are electrostatically complexed to the surface of the micelle with the remainder of the excess counterions in a region around the micelle known as the electrical diffuse double layer as shown in Figure II.19 (17,23.52.53). The absolute electrical potential declines with distance from the micelle surface and approaches zero as the distribution of negative and positive charges become equal far away from the surface. 


\subsubsection{The Effect of Counterion Characteristics on Counterion Binding}

In general the higher the valence of the counterion, the higher the degree of counterion binding; for example, it has been shown that the adsorption of calcium, a divalent ion, has a higher relative adsorption than sodium, a monovalent ion (23).

\subsubsection{Separation of Multivalent Counterions}

Ions in solution can form complexes with surrounding water molecules. This interaction is generally of the ion-dipole type where a positively charged ion interacts with the oxygen portion of the water molecule. A negatively charged ion interacts with one of the hydrogens of the water molecule. The radius of the soluble complex is referred to as the radius of hydration. The radius of hydration has only a slight effect on the interaction of the ion within the Stern Layer with the surface of the micelle (34.54).

\subsubsection{Separation of Multivalent Ions}

Preferential counterion binding of multivalent ions at the micelle surface allows the concentration of those ions on the retentate side of membranes used in semiequilibrium dialysis or ultrafiltration. Figure II.20 shows how the micelle along with counterions electrostatically bound to the 
surface of the micelle are concentrated and the unassociated ions and unaggregated surfactant monomers pass through the membrane in an aqueous solution to the permeate side of the membrane. The selectivity for the higher valence counterion in the MEUf process is directly related to the preferential binding of the counterion to the micelle surface as discussed in section 2.5.1.

\subsubsection{Oosawa Model Applied to MEUF}

Christian et al (19) have shown that the polyelectrolyte theory of Oosawa (55) is very effective in describing the resultant MEUF permeate concentration of a target multivalent counterion by considering the counterion binding to the micelle. The model revolves around the concept that those multivalent counterions electrostatically bound to the surface of the micelle are rejected by the membrane while unbound ions are able to pass through the membrane unimpeded. Therefore, the concentration of each species of the unbound ions is the same for both the retentate and permeate at equilibrium and the thermodynamic activity of each ion pair is the same across the membrane.

In the absence of added monovalent electrolyte, for an anionic surfactant, the binding of the monovalent counterion $\left(\mathrm{M}^{+}\right)$and a divalent counterion $\left(D^{2+}\right)$ to the micelle are described by Eq. (2.10) and Eq. (2.11) respectively (18): 


$$
\begin{gathered}
\ln \frac{\left[M^{+} b_{b}\right.}{\left[M^{*}\right]_{u}}=\ln \frac{\phi}{1-\phi}+P(1-\beta)\left(1-\phi^{\frac{1}{3}}\right) \\
\ln \frac{\left[D^{2+}\right]_{b}}{\left[D^{2+}\right]_{u}}=\ln \frac{\phi}{1-\phi}+2 P(1-\beta)\left(1-\phi^{\frac{1}{3}}\right) \\
\beta=\frac{\left[M^{+} b_{b}+2\left[D^{2+} b_{b}\right.\right.}{\left[A S^{-}\right]_{m c}}
\end{gathered}
$$

where $\beta$ is fraction of the micellar charge neutralized by counterions, $b$ and $u$ are bound and unbound counterions, $\phi$ is the fraction of the total volume within which the bound counterions are located, $\left[A S^{-}\right]_{\text {mic }}$ is the anionic surfactant concentration in micellar form, and $P$ is a dimensionless parameter.

The concentration of negatively and positively charged ions in the permeate, as well as the retentate, must be equal in order to maintain electroneutrality as shown by Eq. (2.13) and Eq. (2.14):

$$
\begin{aligned}
& 2\left[D^{+2}\right]_{r o t}+\left[M^{+}\right]_{r o t}=\left[A S^{-}\right]_{m o t}+\left[C O^{-}\right]_{r o t} \\
& 2\left[D^{+2}\right]_{p o r}+\left[M^{+}\right]_{p o r}=\left[A S^{-}\right]_{p o r}+\left[C O^{-}\right]_{p o r}
\end{aligned}
$$


where ret and per represent the retentate and permeate total individual ion concentrations in those solutions respectively and $\mathrm{Co}^{-}$is the co-ion of the divalent cation (e.g., $\mathrm{Cl}^{-}$).

Under equilibrium conditions, the following relations are relevant for unassociated ion species (18):

$$
\begin{aligned}
& {\left[D^{2+}\right]_{\mathrm{rol}}\left[\mathrm{M}^{-}\right]^{2} \mathrm{wr}=\left[D^{2+}\right]_{\mathrm{per}}\left[\mathrm{Co}^{-}\right]_{\mathrm{mrr}}^{2}} \\
& {\left[D^{2+}\right]_{m o t}\left[A S^{-}\right]^{2}{ }_{m o t}=\left[D^{2+}\right]_{m o r}\left[A S^{-}\right]^{2} \mathrm{mr}} \\
& {\left[\mathrm{M}^{+}\right]_{\mathrm{rot}}\left[\mathrm{Co}^{-}\right]_{\mathrm{nt}}=\left[\mathrm{M}^{+}\right]_{\mathrm{mrr}}\left[\mathrm{Co}^{-}\right]_{\mathrm{mr}}}
\end{aligned}
$$

The surfactant in the retentate is either monomer or aggregated in surfactant micelles. The concentration of anionic surfactant in the micellar form may be determined by the following relationship (19):

$$
\beta\left(\ln \left(\left[M^{+}\right]_{u}+2\left[D^{2+}\right]_{u}\right)\right)+\ln \left[A S^{*}\right]_{\text {mon }}=(\ln (C M C))(1+\beta)
$$

where $C M C$ is the critical micelle concentration for the surfactant without any additional electrolyte added to the solution and $\left[A S^{-}\right]_{\text {mon }}$ is the anionic surfactant monomer concentration present. 
Counterion concentration in the retentate, including that from added monovalent electrolyte, affects the degree of counterion binding of the target multivalent ion with the micelle. The parameter $\boldsymbol{P}$ accounts for the added monovalent electrolyte by the following relationship (18):

$$
P=\frac{P^{0}}{1+\alpha\left[M^{+} \mathrm{Co}^{-}\right]^{\frac{1}{2}}}
$$

where $\left[\mathrm{M}^{+} \mathrm{Co}^{-}\right]$is the concentration of the added monovalent electrolyte, the $M^{+}$cation is the same as that from the salt of the anionic surfactant and the $\mathrm{Co}^{-}$anion is is the total anion concentratiopn. $\mathrm{PO}$ is determined from experiments without added monovalent electrolyte. The parameter $\alpha$ is determined experimentally from the permeate solution resulting from a retentate solution with added electrolyte. $P O$ and $\alpha$ depend on the type of metal ion (55).

The model accurately represents experimental data except at high metal concentrations or high salinities where the experimental permeate target multivalent ion concentrations are lower than the predicted values (18).

\subsection{Activated Carbon}

Activated carbon, which is widely used to separate organic compounds from vapor and liquid streams, is produced from wood, coal, 
peat, shells, etc. Considering the materials activated carbon is produced from, it is not surprising that the activated carbon surface is very heterogeneous with both charged and nonpolar adsorption sites. The surfactant interaction with the carbon surface is dependent on the characteristics of the adsorption sites on the surface. Surfactants can adsorb at the solid-liquid interface by several mechanisms, two of the most important being hydrophobic bonding and electrostatic interaction. Electrostatic interactions between the surfactant and the solid surface require the charged head group of the surfactant to have an opposite charge to the adsorption site on the surface. The adsorption occurs with the charged head group oriented toward the oppositely charged adsorption site on a surface as shown in Figure II.21. Hydrophobic bonding of surfactant tails allows the formation of a second layer of adsorbed surfactant with the charged head groups oriented toward the polar solvent and the tail groups of the second layer of surfactant having a hydrophobic interaction with the tail groups of the surfactants electrostatically bound on the surface, resulting in the formation of bilayer aggregates known as admicelles. The other mechanism for adsorption at the solid-liquid interface is hydrophobic bonding between a surface and the hydrophobic tail group of the surfactant as illustrated in Figure II.21. Given the heterogeneity of the surface of activated carbon, both electrostatic and hydrophobic bonding likely occur on the carbon surface. 


\subsubsection{Granular Activated Carbon Beds}

Typically the solution or vapor with the target organic solute to be removed is passed through a packed bed of granulated activated carbon. This is known as a fixed bed adsorber. As the solution flows through the column, the target organic compound adsorbs on the surface of the activated carbon. There is a finite amount of activated carbon in a packed bed and therefore, a finite surface area on which the target organic solute can adsorb. The solution with the target organic solute enters the inlet of the packed bed and adsorbs on the activated carbon near the inlet. The available adsorption sites near the inlet portion of the column are covered with the target organic solute causing the adsorption zone, known as the mass transfer zone, to shift farther down the column toward the packed bed outlet as shown in Figure II.22. Eventually all of the adsorption sites are covered and the activated carbon can no longer adsorb additional target organic solute from the feed solution. 


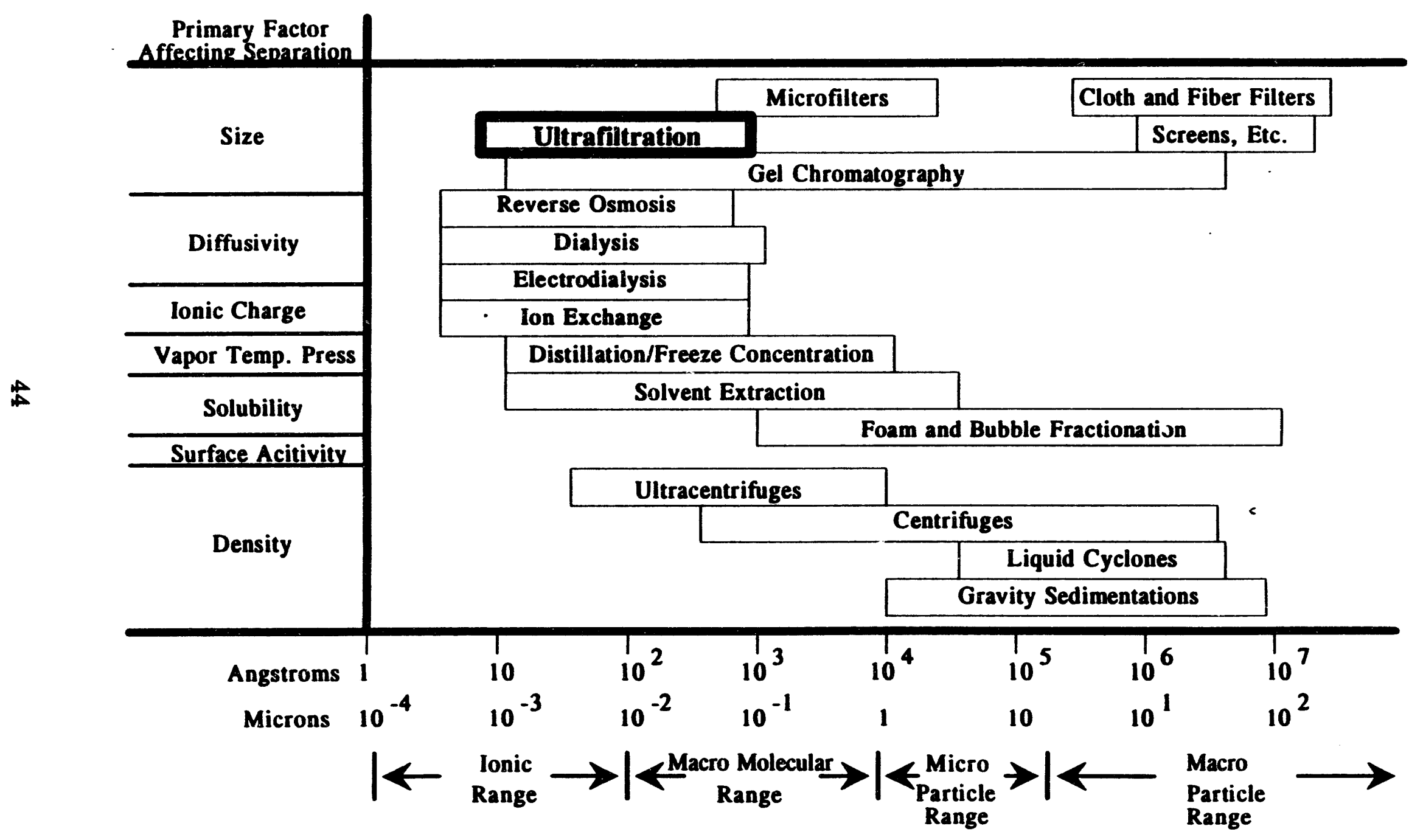

FIGURE II.1 Overview of a Variety of Separation Techniques, the Range of Separation and the Primary Factor Affecting the Separation (28) 


\begin{tabular}{|c|c|c|c|}
\hline PROCESS & DRIVING FORCE & PERMEATE & RETENTATE ${ }^{*}$ \\
\hline OSMOSIS & CHEMICAL POTENTIAL & WATER & SOLUTES \\
\hline DIALYSIS & $\begin{array}{l}\text { CONCENTRATION } \\
\text { DIFFERENCE }\end{array}$ & $\begin{array}{c}\text { WATER + } \\
\text { SMALL MOLECULES }\end{array}$ & $\begin{array}{c}\text { LARGE } \\
\text { MOLECULES }\end{array}$ \\
\hline ULTRAFILTRATION & PRESSURE & $\begin{array}{c}\text { WATER + } \\
\text { SMALL MOLECULES }\end{array}$ & $\begin{array}{l}\text { LARGE } \\
\text { MOLECULES }\end{array}$ \\
\hline REVERSE OSMOSIS & PRESSURE & WATER & SOLUTES \\
\hline ELECTRODIALYSIS & E.M.F. & $\begin{array}{c}\text { WATER + } \\
\text { IONIC SOLUTES }\end{array}$ & $\begin{array}{l}\text { NONIÓNIC } \\
\text { SOLUTES }\end{array}$ \\
\hline MICROFILTRATION & PRESSURE & $\begin{array}{c}\text { WATER + } \\
\text { DISSOLVED ORGANICS }\end{array}$ & $\begin{array}{c}\text { LARGE } \\
\text { SUSPENDED } \\
\text { PARTICLES }\end{array}$ \\
\hline *Includes Water & & & \\
\hline
\end{tabular}

FIGURE II.2 Characteristics of Common Membrane Separation Processes (28) 


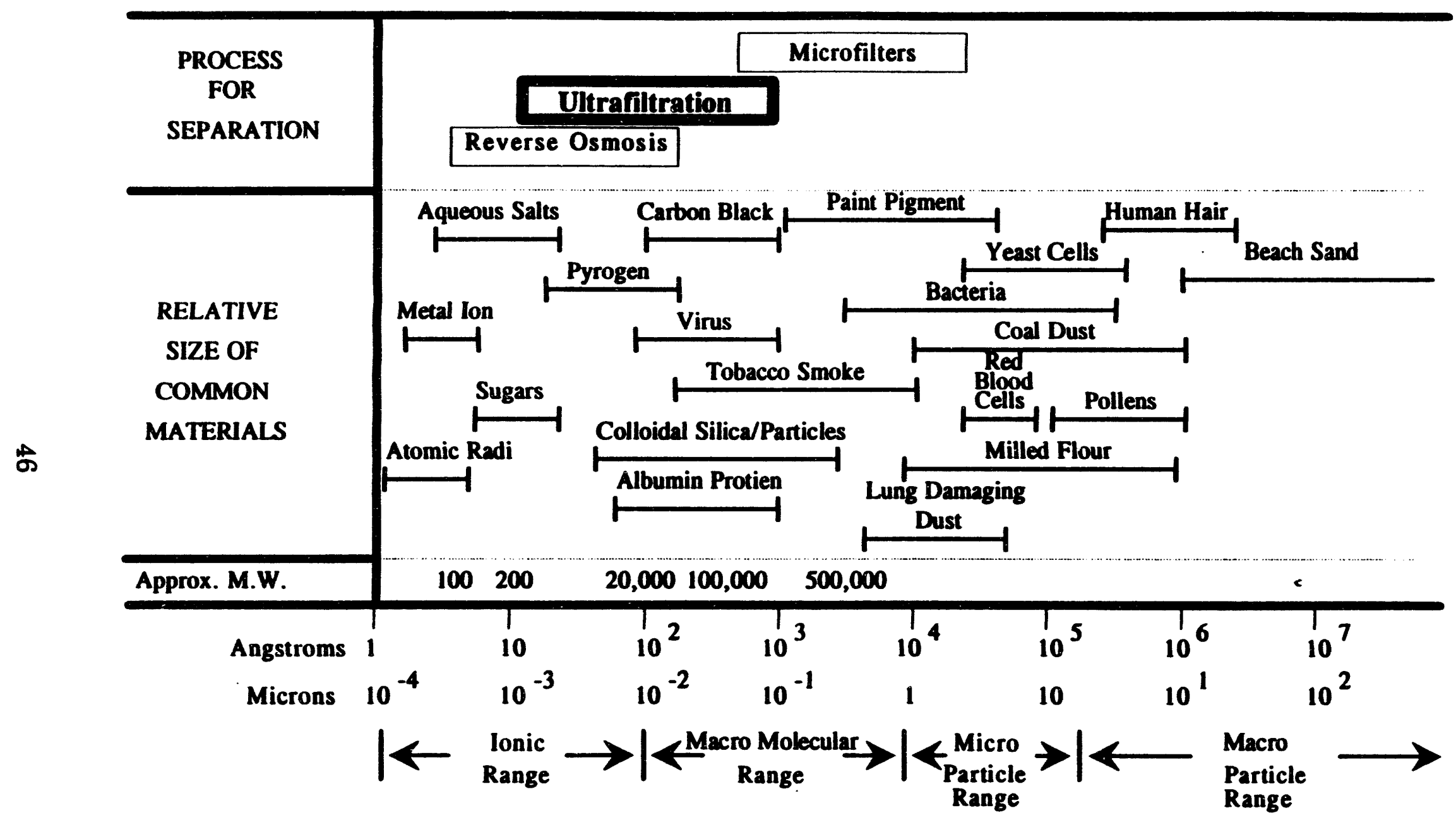

FIGURE II.3 The Relationship of the Size of Common Materials and Approximate Molecular Weights to the Range of Application for Pressure Driven Membrane Separation Processes (28) 


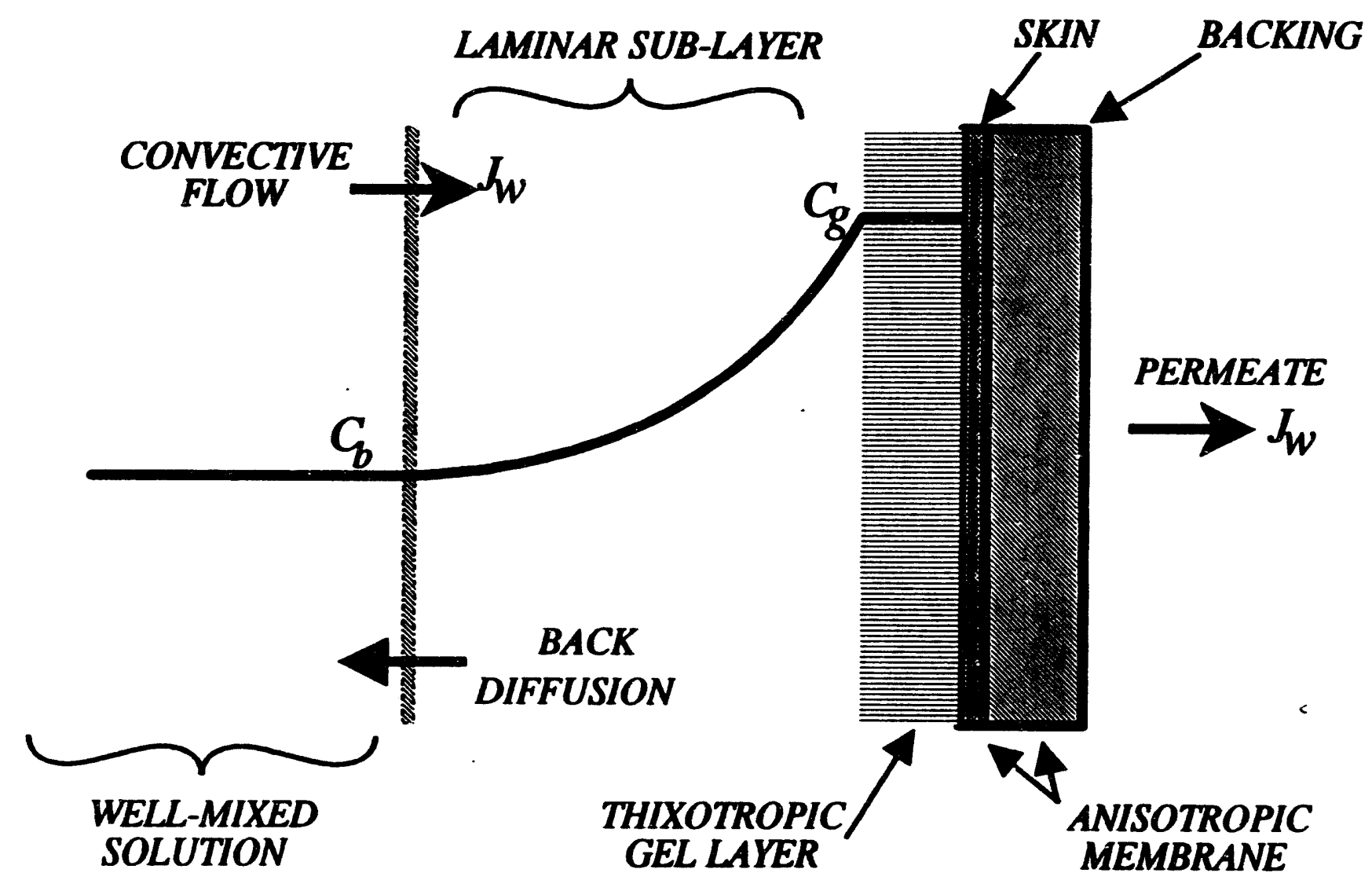

FIGURE II.4 Illustration of the Concept of Concentration Polarization with Gel Polarization of Macromolecules and Colloids During the Ultrafiltration Process 


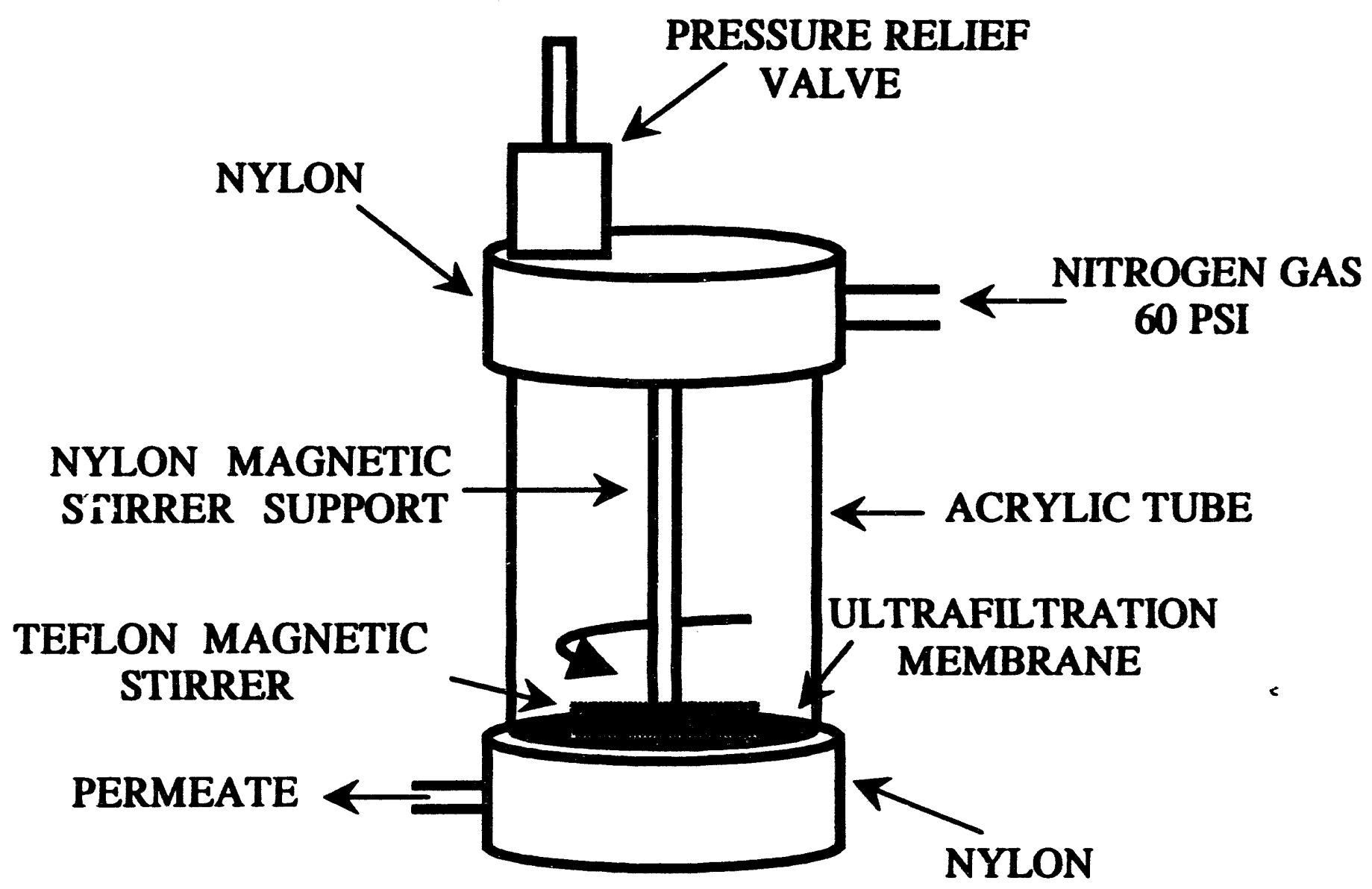

FIGURE II.5 Illustration of Stirred Cell and Major Components 

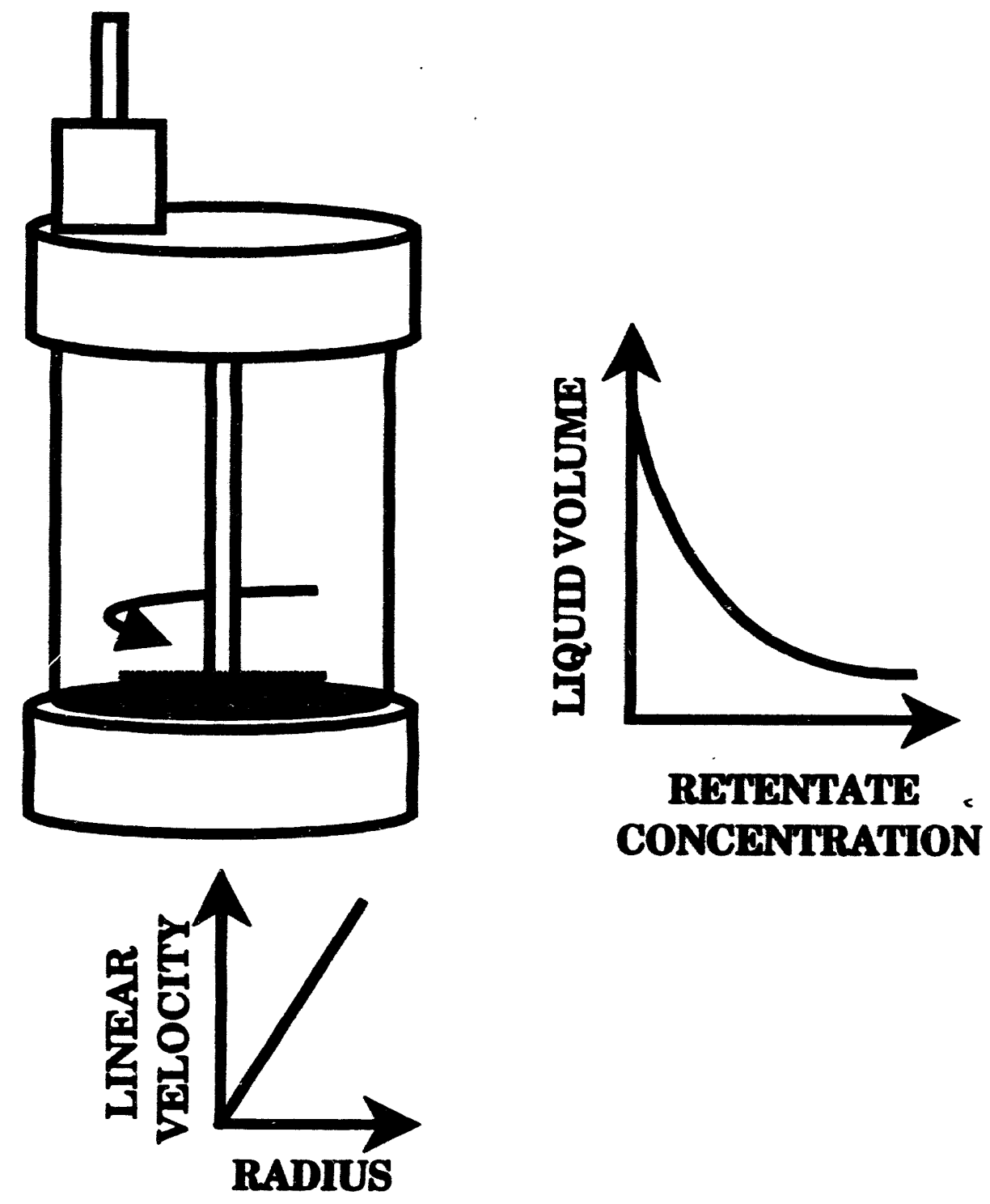

FIGURE II.6 Operational Characteristics of Stirred Cell 


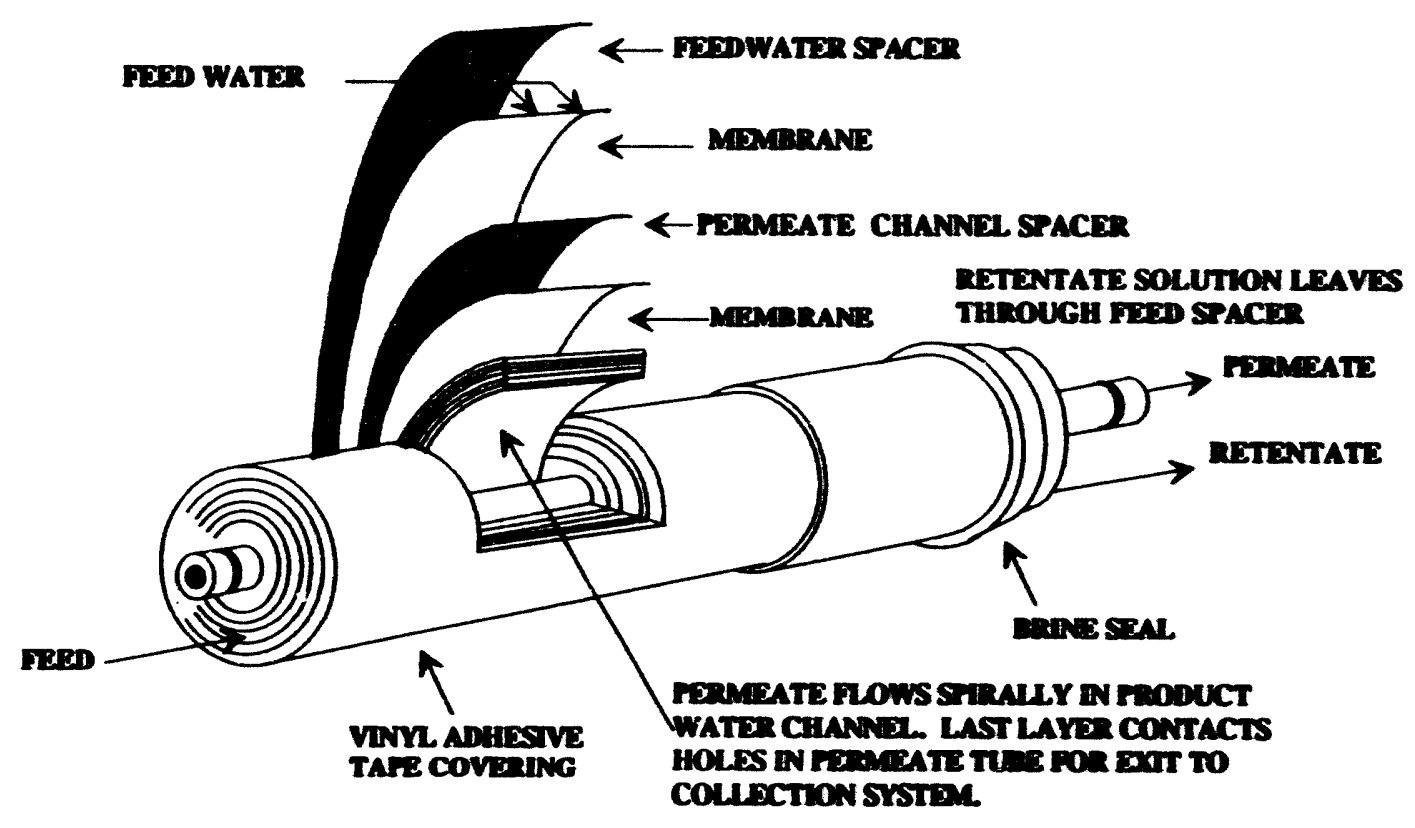

FIGURE II.7 Illustration of the Geometry and Major Components of a Spiral Wound Ultrafiltration Membrane 

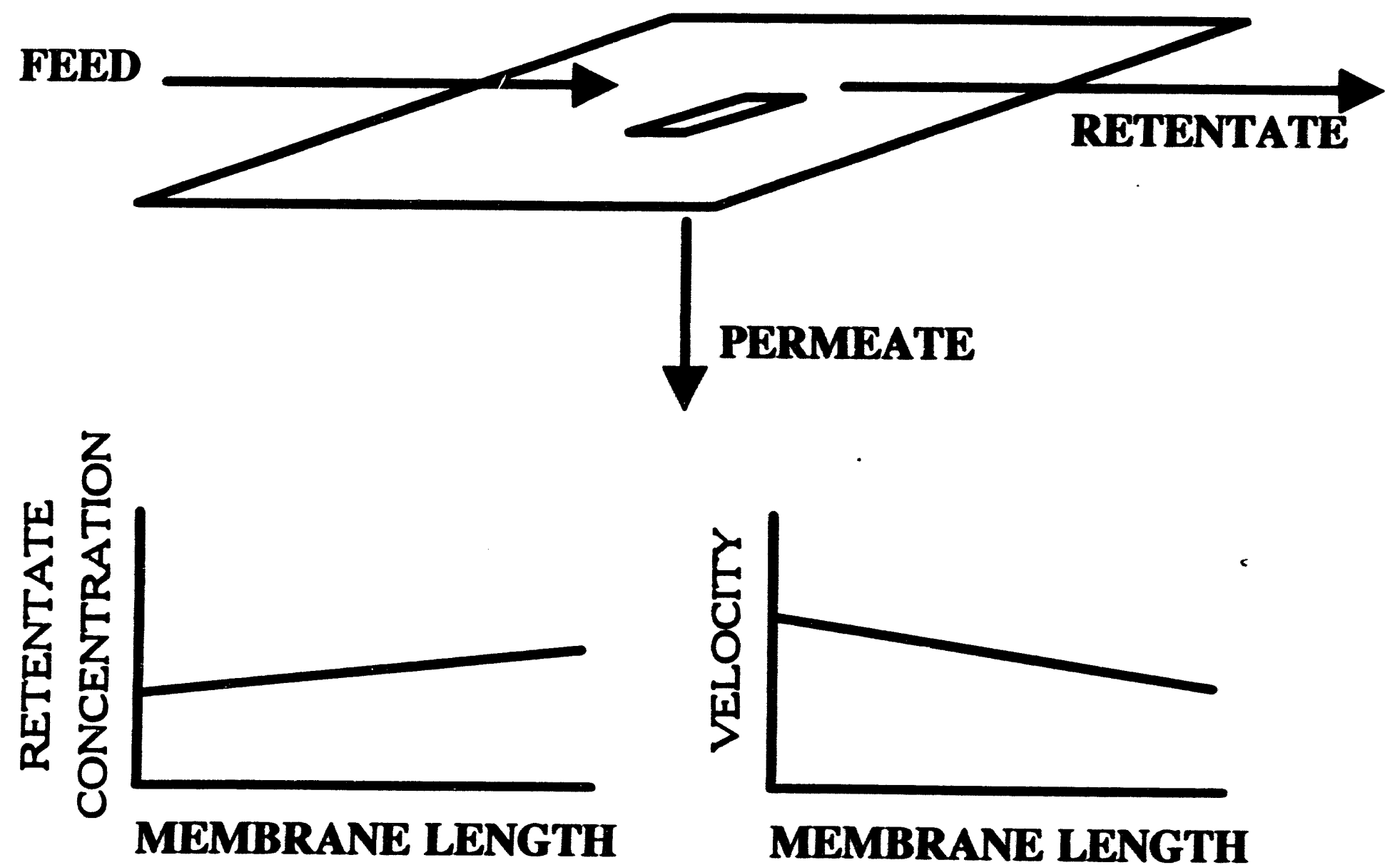

FIGURE II.8 Operational Characteristics of Spiral Wound Membrane 


\section{SCHEMATIC OF SURFACTANT MONOMER}

(SODIUM DECYL SULFATE)

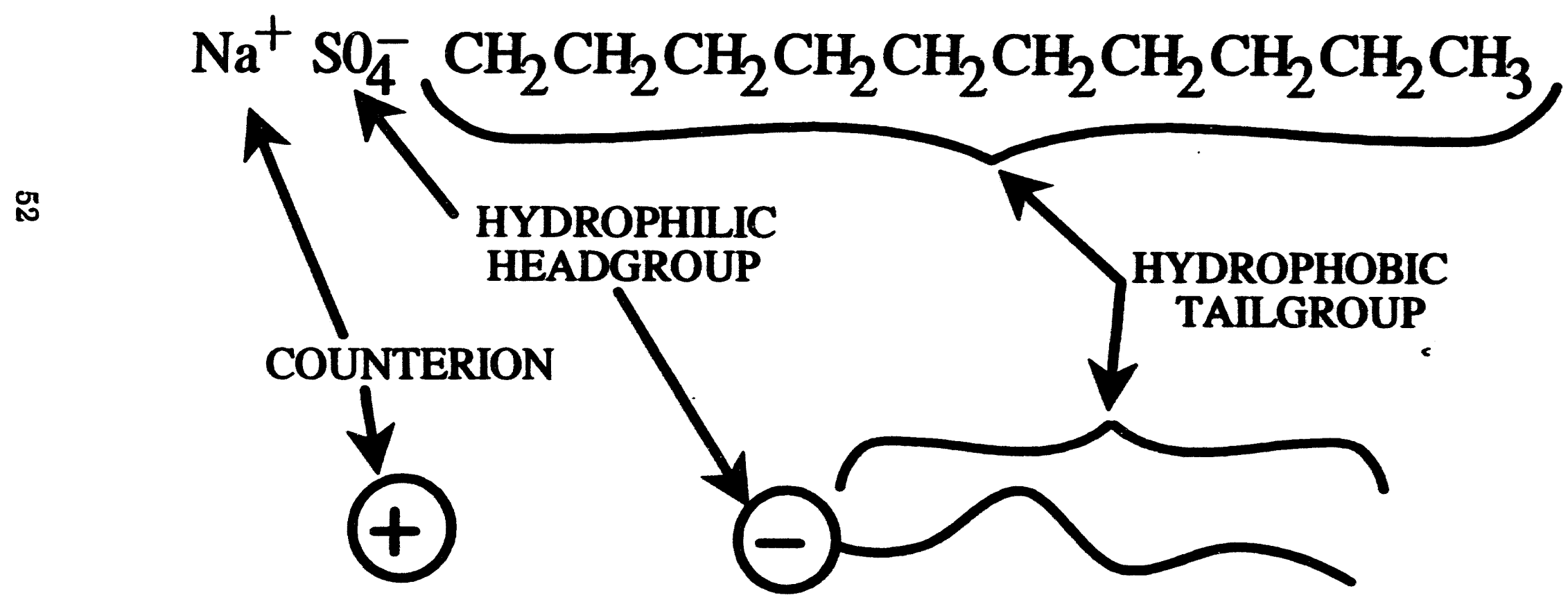

FIGURE II.9 Illustration of a Surfactant Monomer and the Hydrophobic and Hydrophilic Regions 


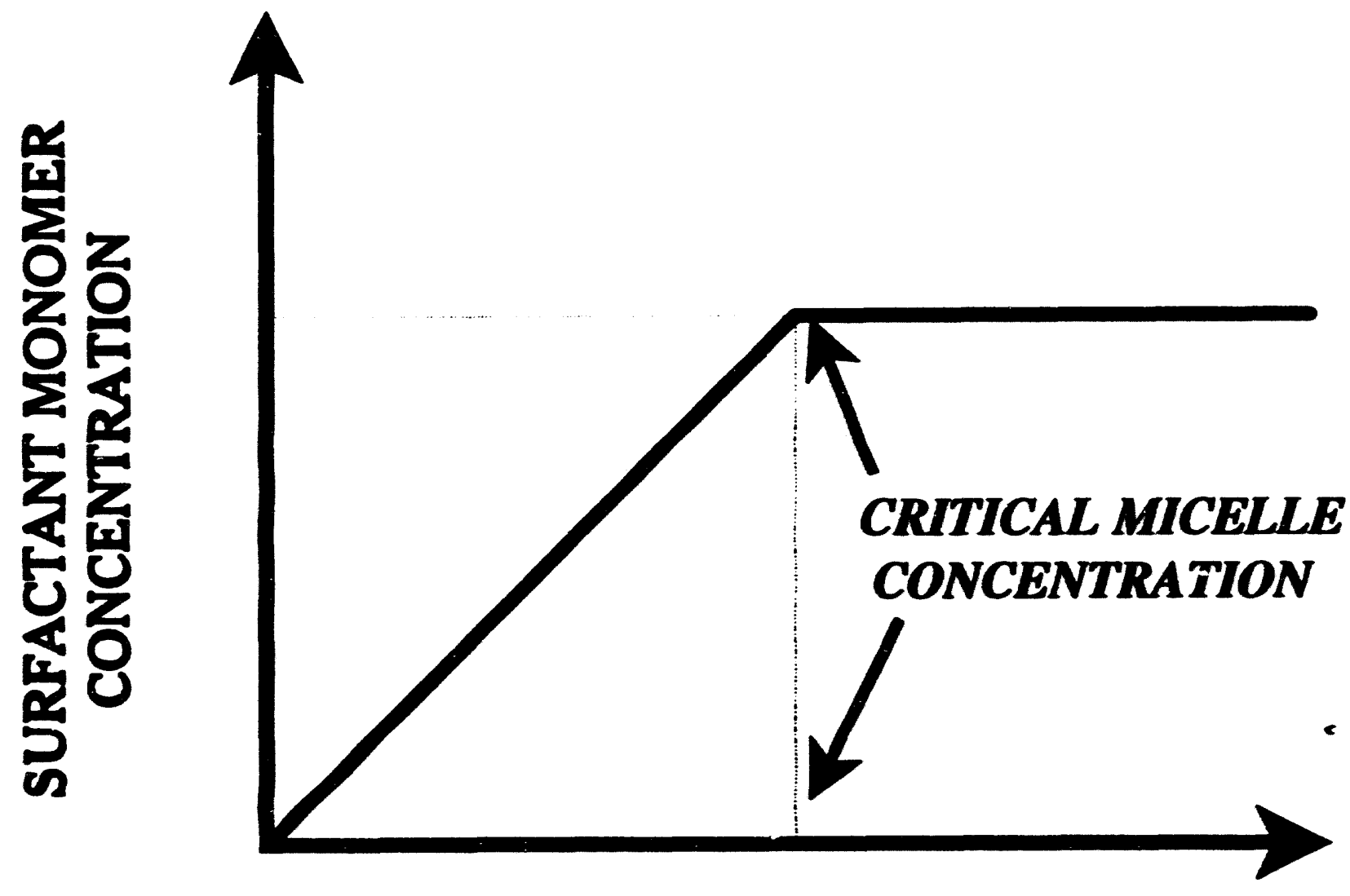

\section{TOTAL SURFACTANT CONCENTRATION}

FIGURE II.10 Relationship of Total Surfactant Concentration, Monomer Concentration and Critical Micelle Concentration 


\section{SCHEMATIC OF MICELLE IN AN AQUEOUS SOLUTION}

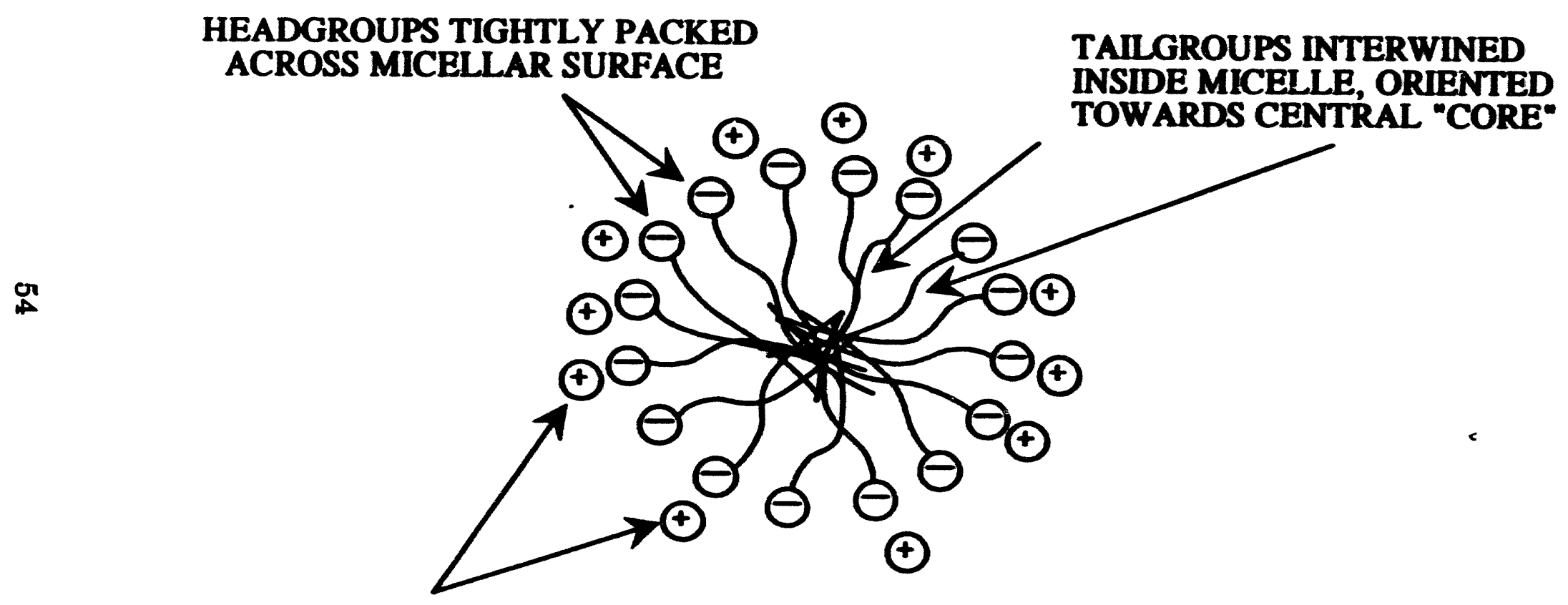

HEADGROUP COUNTERION PARTIALLY ASSOCIATES ON SURFACE OF IONIC SURFACTANT MICELLES

FIGURE II.11 Typical Micelle Composed of Anionic Monomers in an Aqueous Solution 


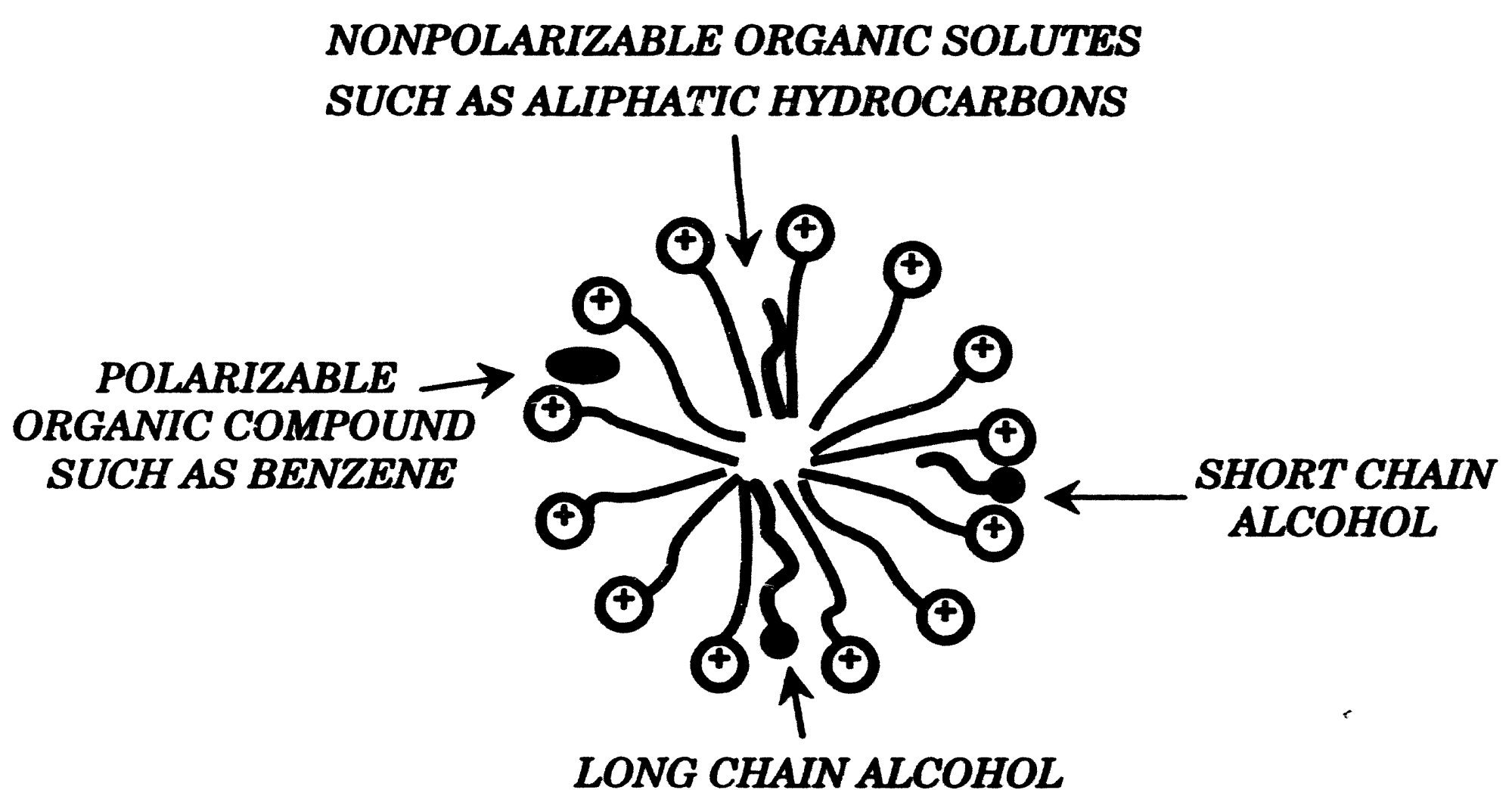

FIGURE II.12 Regions Where Different Types of Organic Solutes Solubilize within a Surfactant Micelle. 


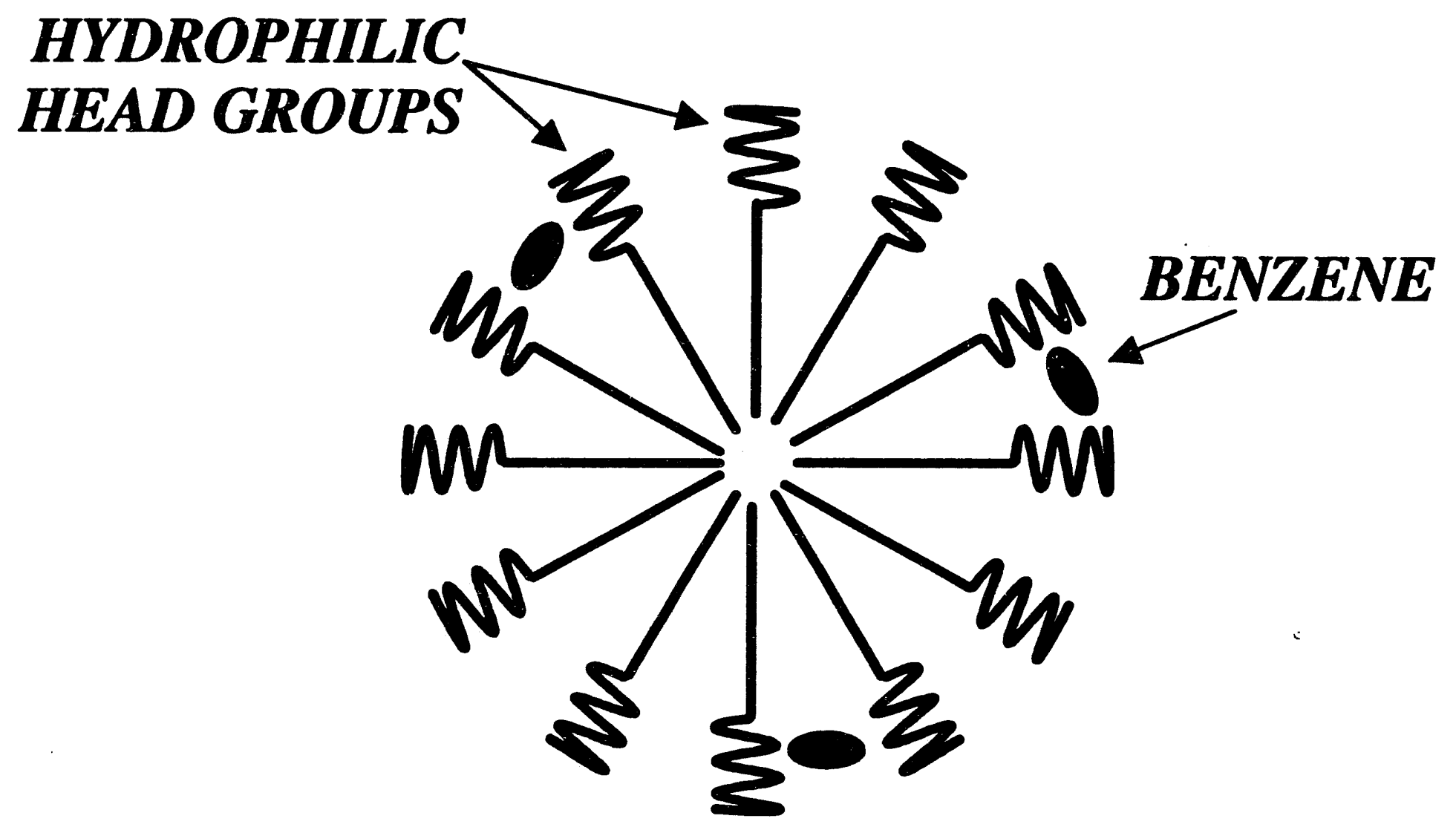

FIGURE II.13 Solubilization Location of Benzene Between the Hydrophilic Groups of Polyethoxylated Nonionic Surfactant Micelle 


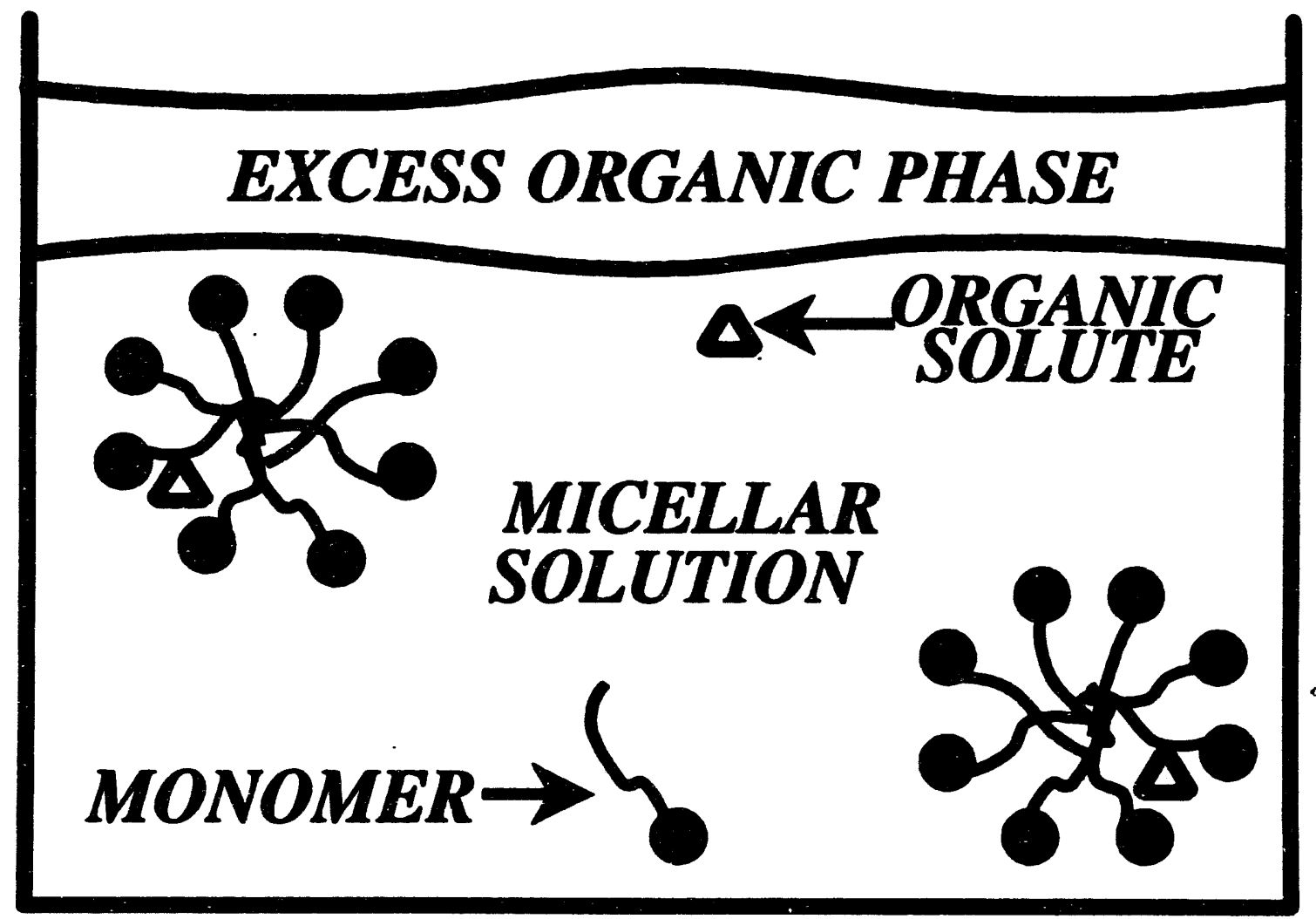

FIGURE II.14 Maximum Additivity Method for Determination of Solubilization Constant 


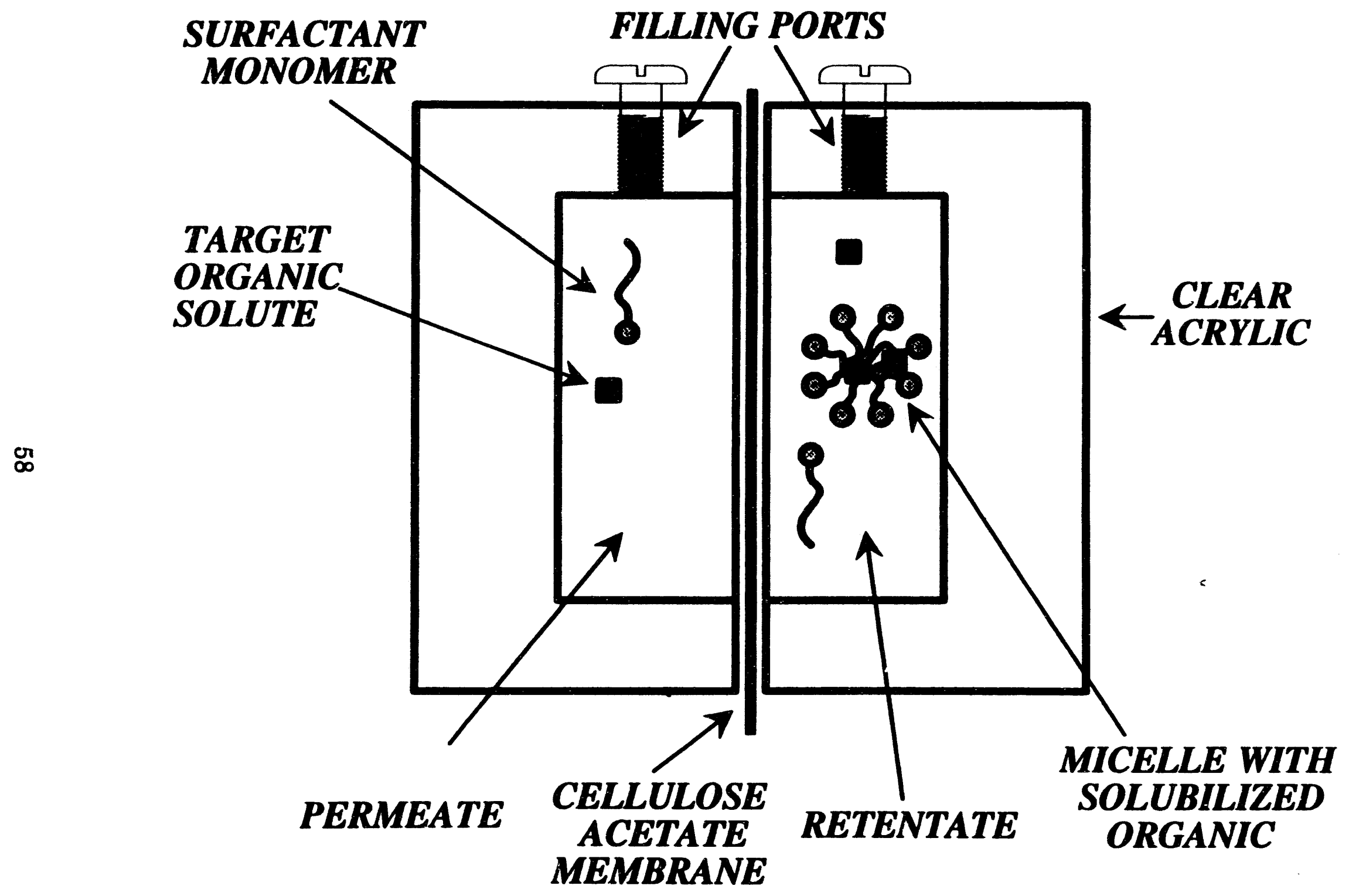

FIGURE II.15 Semiequlibrium Dialysis Cell Used For Solubilization Constant Determination 


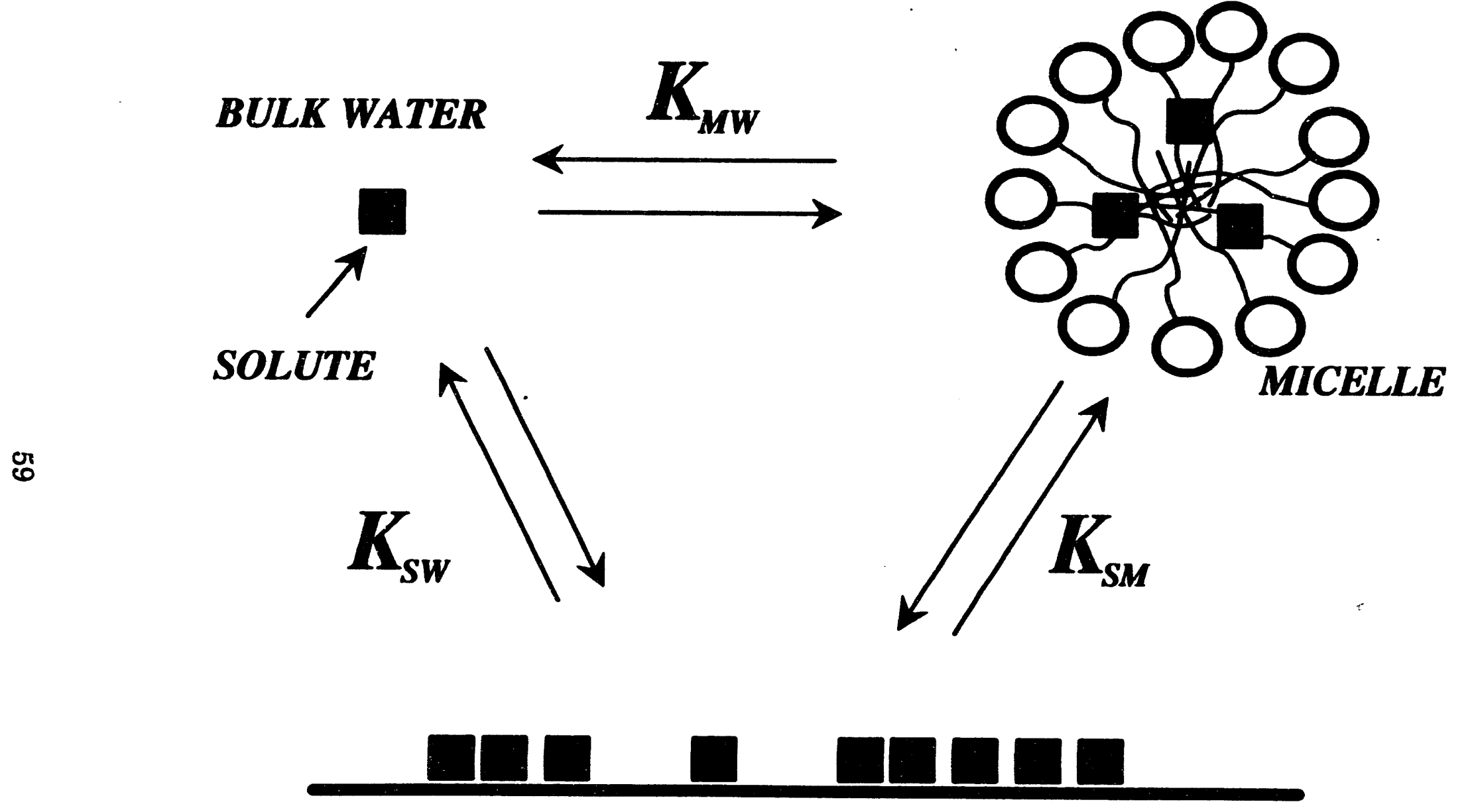

\section{STATIONARY PHASE}

FIGURE II.16 The Partition of Organic Solutes Between the Micellar Phase and the Solvent in Micellar Mobile Phase Chromatography for the Measurement of Solubilization Constant 
$\oplus$

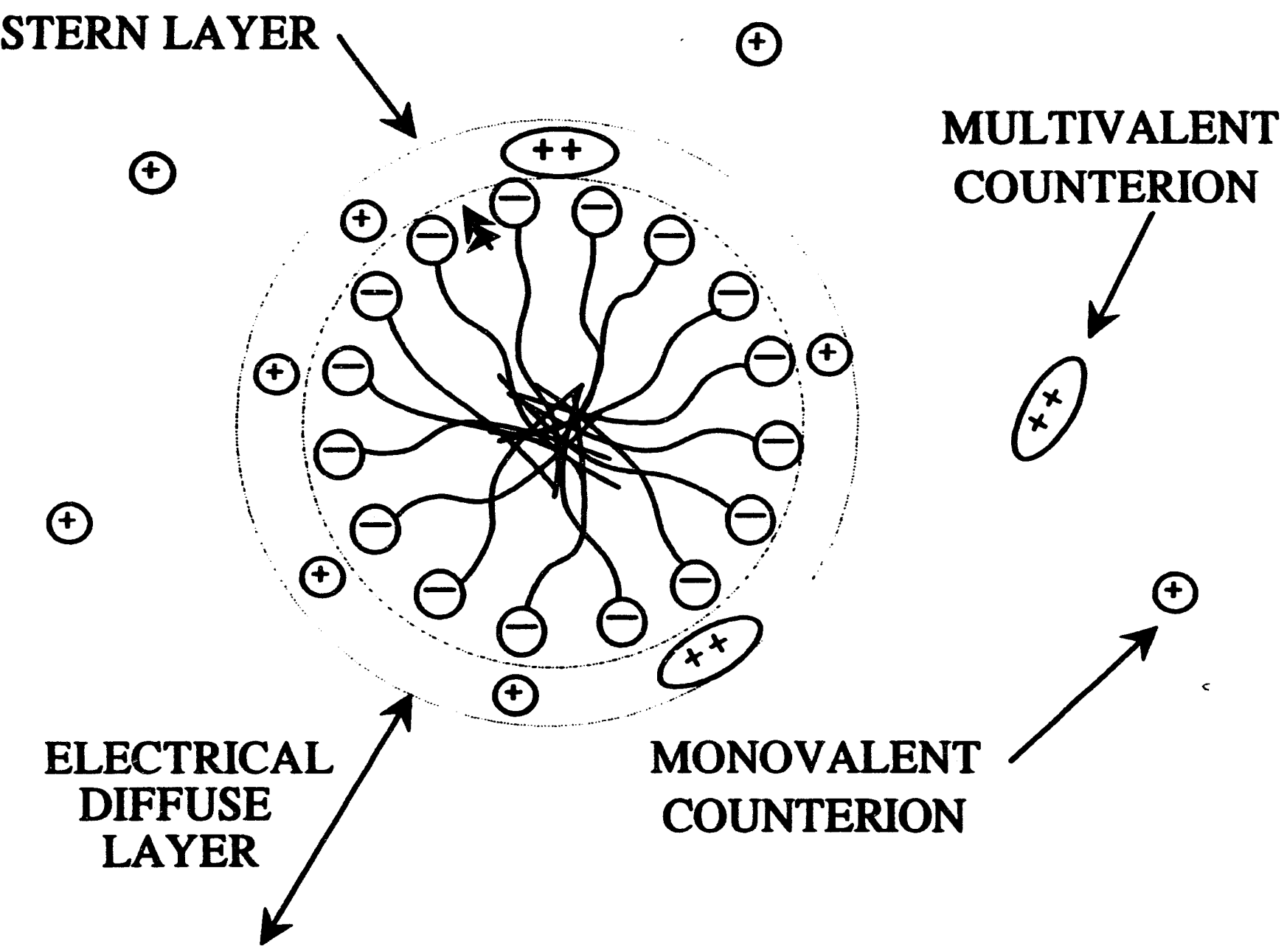

FIGURE II.17 Counterion Adsorption at the Micelle Aqueous Interface 


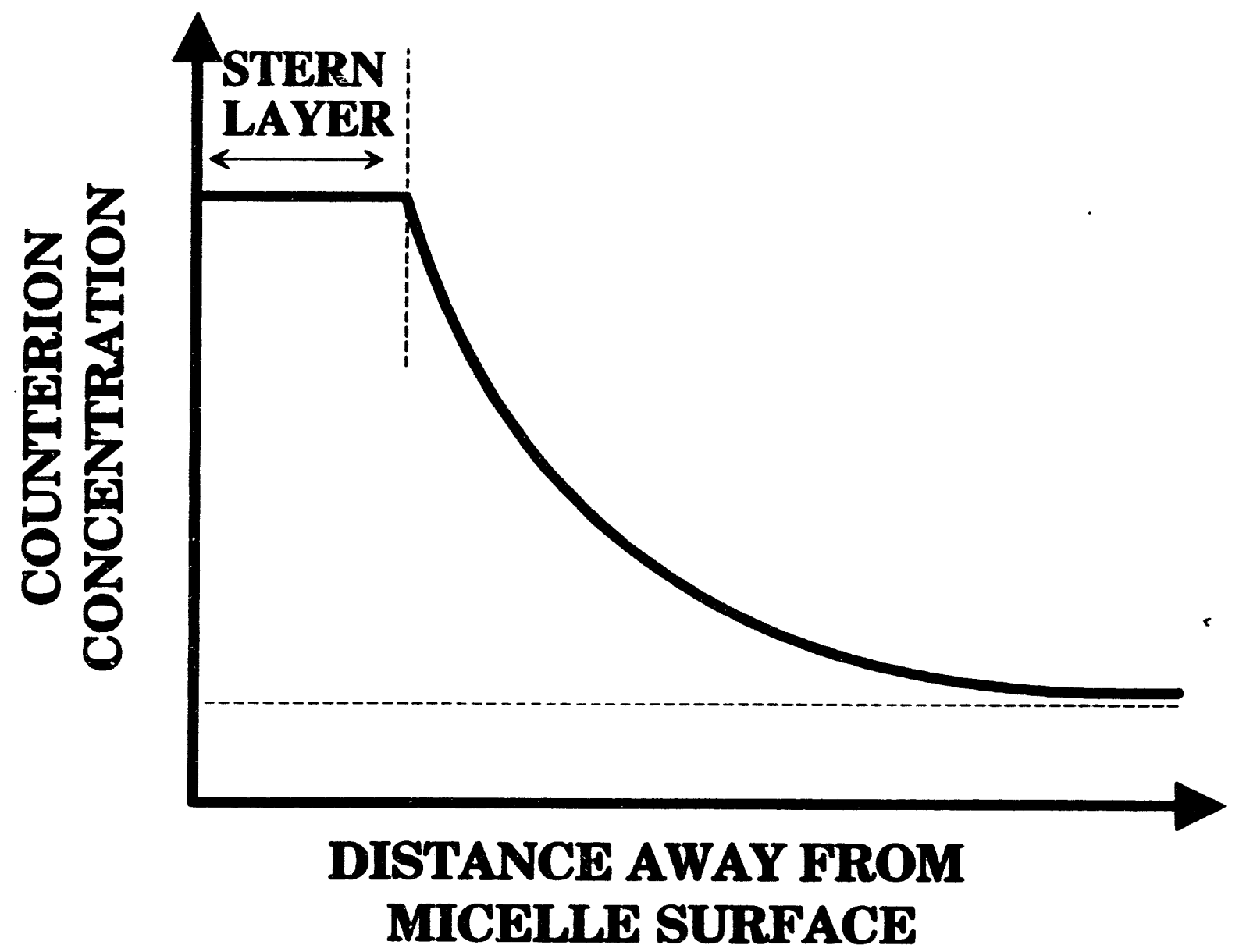

FIGURE II.18 Counterion Concentration Profile Extending from the Micelle Surface 

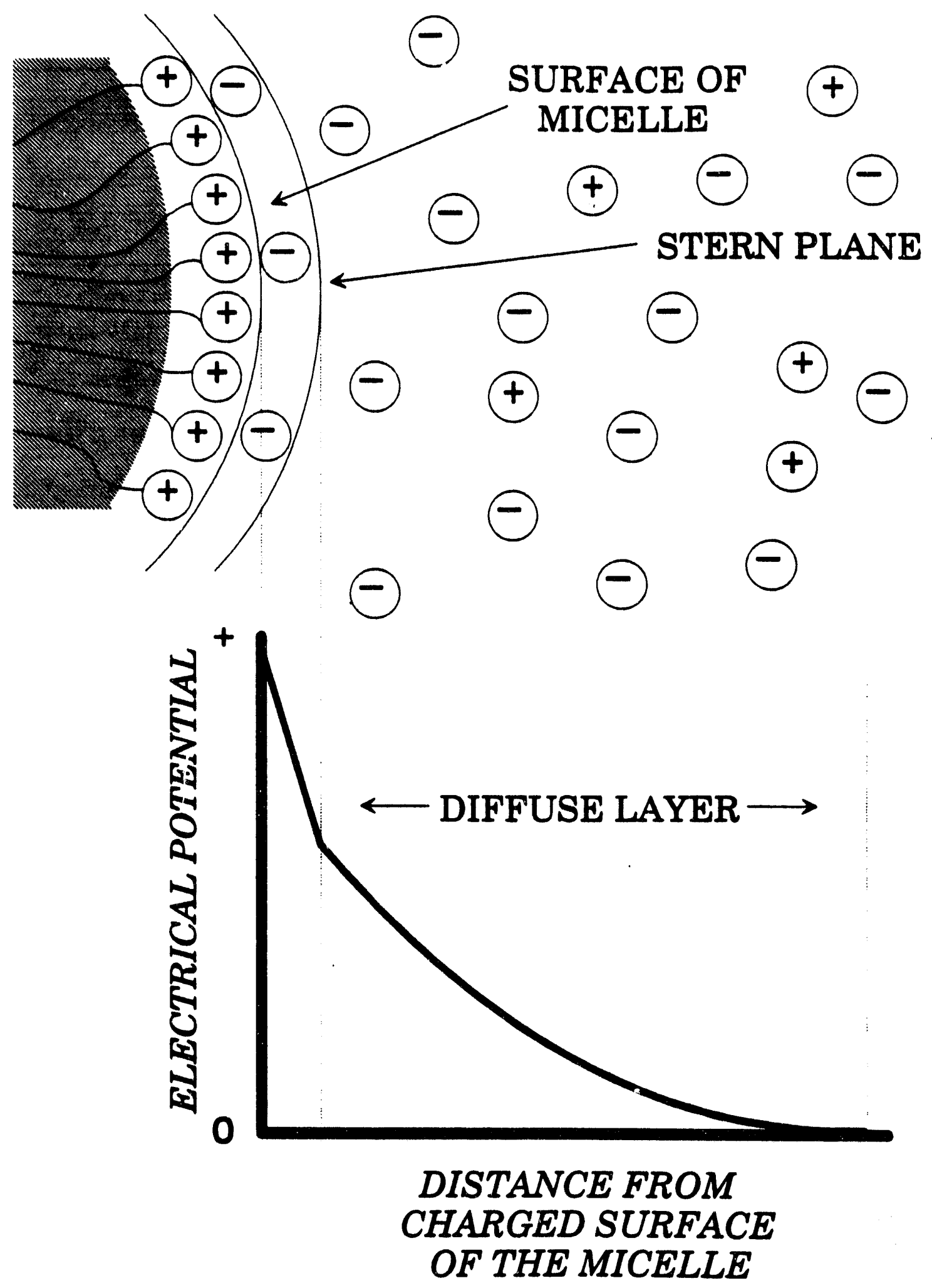

FIGURE II.19 Illustration of Stern Model of the Electrical Double Layer 


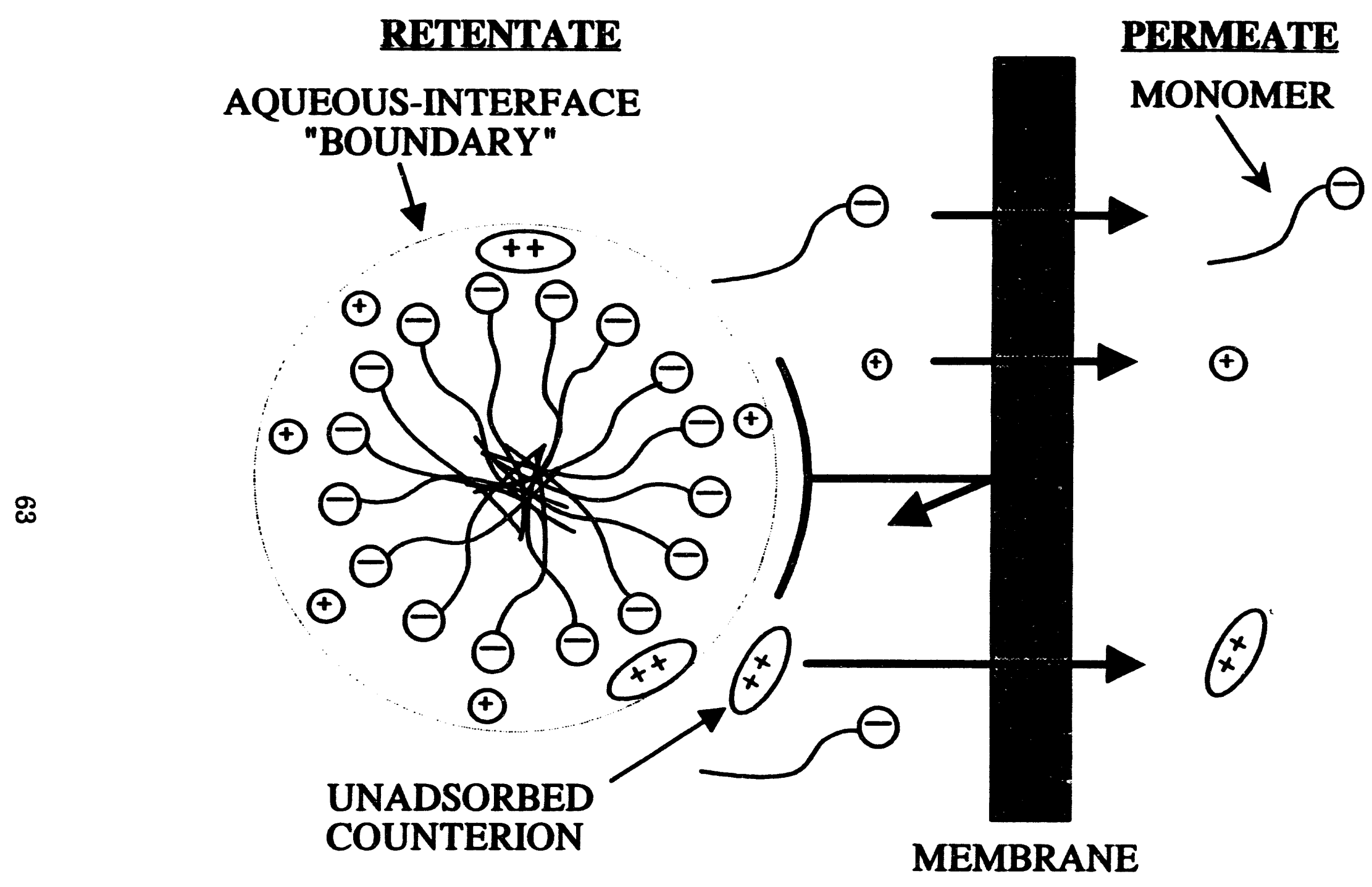

FIGURE II.20 Separation of Multivalent Counterions in MEUF 


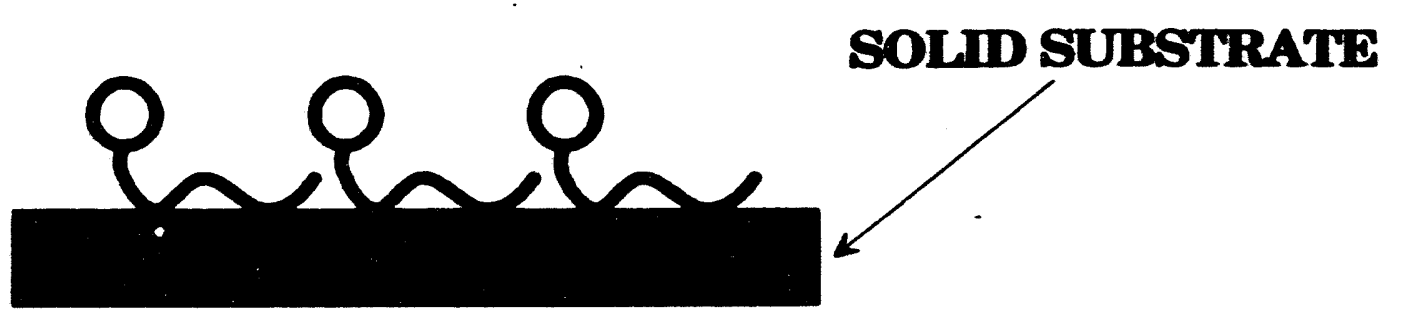

SURFACTANT ADSORPTION ON A HYDROPHOBIC SURFACE

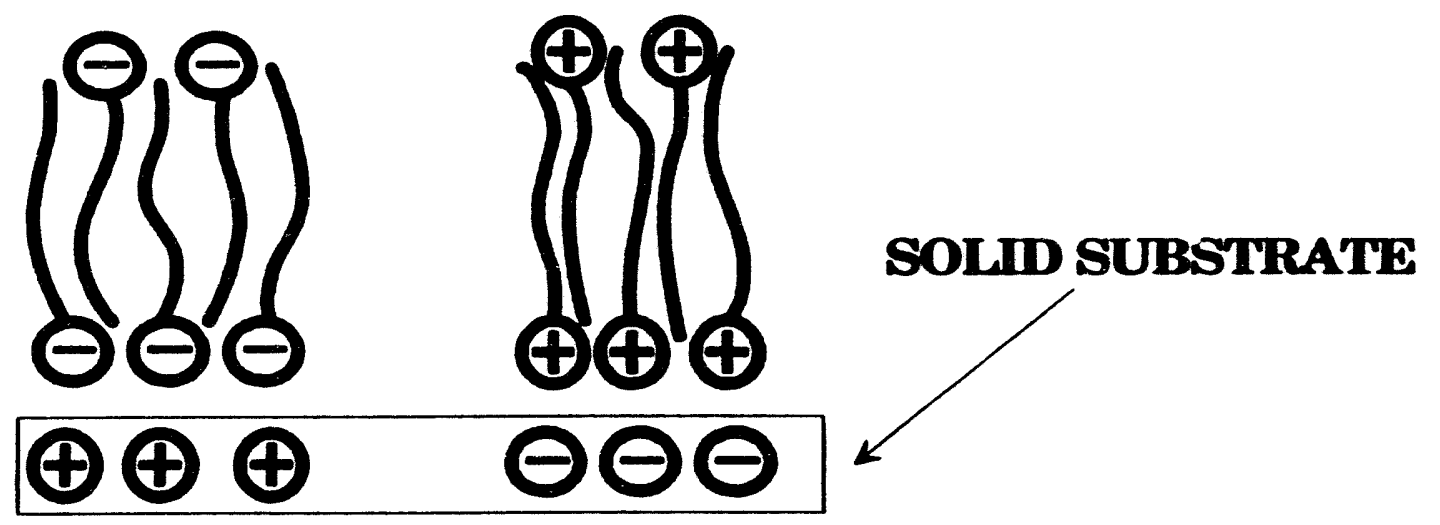

SURFACTANT ADSORPTION ON A CHARGED SURFACE

FIGURE II.21 Some Different Modes of Adsorption at the Solid-Liquid Interface 

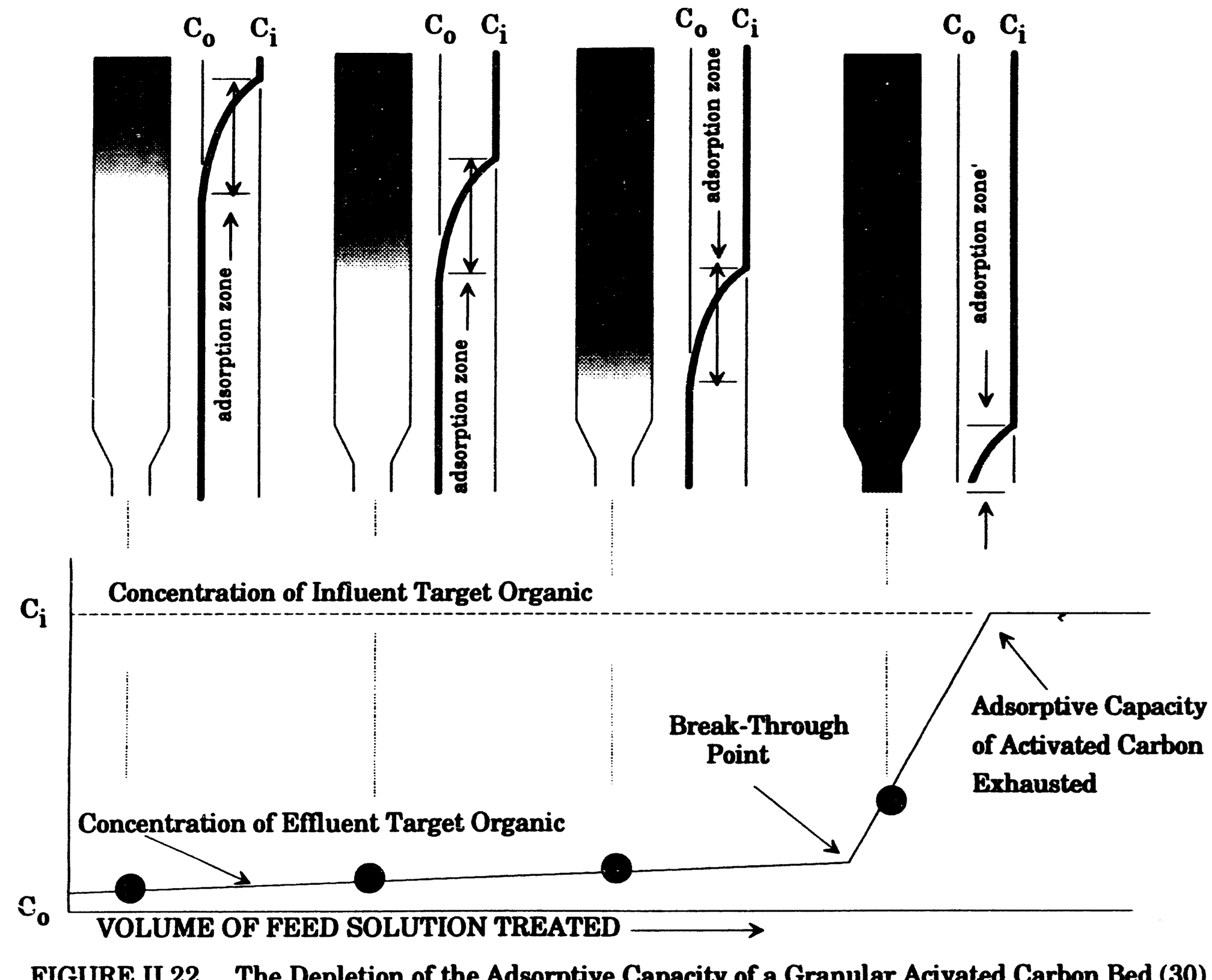


\section{CHAPTER 3}

\section{MXPERIMINTAL PROCEDURES}

The experiments are divided into two basic categories: 1) SECR for regenerating activated carbon used for vapor and liquid phase applications (Chapter $4 \& 5$ ); and 2) MEUF for the recovery of nonvolatile target organics and target metals (Chapter 6 ) and the recovery of volatile target organics (Chapter 7).

The studies presented in Chapter 4 investigated the regeneration of activated carbon on which amyl acetate and toluene was adsorbed by using a micellar flood of sodium dodecyl sulfate (SDS). The studies presented in Chapter 5 investigated the regeneration of activated carbon on which 4-tert-butylphenol (TBP) was adsorbed by using a micellar flood of cetyl pyridinium chloride (CPC). The studies presented in Chapters 6 investigated the recovery of TBP, copper and chromate by MEUF using SDS and CPC. The studies presented in Chapter 7 investigated the recovery of 1,1,1- trichloroethane (111TCE), tetrachloroethylene (PCE), dichloromethane (DCM) and trichloroethylene (TCE) by MEUF using a variety of surfactants.

\subsection{SECR Vapor Phase Experiments}

Shown in Figures III.1 through III.5 are the configurations of the experimental apparatus used to study the removal of amyl acetate and toluene from activated carbon. Table III.1 lists the major equipment and 
vendors. A $25 \mathrm{~mm}$ diameter, $1000 \mathrm{~mm}$ long jacketed column with a filter at both ends was used for these experiments. The column contained $200 \mathrm{~g}$ of Calgon PCB 12 X 30 vapor phase granular carbon with a nitrogen BET surface area of 1150-1250 square meters per gram (3). One pore volume in this column was $300 \mathrm{~mL}$. The temperature was maintained at $30^{\circ} \mathrm{C}$ by water circulated through a jacket from a heater-circulator in a constant temperature water bath. A plunger in the column adjusted the carbon bed depth and maintained a fixed bed height. A constant pressure gear pump maintained a constant flow rate as the regenerant fluid or flushing water was pumped through the bed.

The target organic compounds for this study were amyl acetate and toluene, the structures of which are shown in Figure III.15. Both compounds were selected because they are widely used in the painting and printing industry. The amyl acetate concentration in the effluent from the column was analyzed by gas chromatography and the toluene concentration was analyzed using HPLC with a UV detector. The sodium dodecyl sulfate (SDS) concentration was analyzed using HPLC with a conductivity detector.

The amyl acetate and toluene were equilibrated with the carbon by bubbling compressed air through the liquid phase of the organic at room temperature as shown in Figure III.1. 
TABLE III.1

Major Equipment Used for SECR Investigations

\begin{tabular}{|c|c|}
\hline COLUMN & $\begin{array}{c}\text { Altex Glass Chromatography } \\
\text { Column } \\
\text { Rainin Instrument Company } \\
\text { Chromatography Column \#252- } \\
20 \\
\text { Plunger for Column \#252-23 } \\
\text { Water Jacket \#252-29 }\end{array}$ \\
\hline PUMP/MOTOR & Magnetic Drive Gear Pump \\
& Cole Parmer \\
& Pump Head, Cat.\#, N-07002-23 \\
& Pump Motor, Cat.\#, N-07003-90 \\
\hline COMPRESSOR & Double Diaphragm \\
(for organic loading) & Fish Aquarium Pump \\
\hline COMPRESSOR & Diaphragm Pump \\
(for drying) & DeVilbiss Co. \\
& Model No.561547 \\
\hline HEATER/CIRCULATOR & Haake Buchler Instruments \\
& Type \#000-4493 \\
\hline
\end{tabular}

The organic vapor/air mixture was passed through the carbon bed and then to a condenser (cold finger) maintained at $-20^{\circ} \mathrm{C}$ to detect if breakthrough occurred and to protect the elastic polymer parts of the compressor from possible damage due to organic vapors. The air was recycled to the compressor so that all of the vaporized organic was adsorbed on the carbon, making the adsorption level and to make the adsorption level uniform throughout the bed. No organic condensation in the cold fi wer was observed during these operations. The amount of organic adsorbed on the carbon bed ranged from $5 \mathrm{~mL}$ to $20 \mathrm{~mL}$. The 
equilibration time for the toluene was approximately 12 hours and was approximately 24 hours for the amyl acetate. Equilibration was defined as the complete volatilization of the organic in the feed bottle and the absence of organic in the cold finger as determined by visual inspection.

Shown in Figure III.2 is the experimental apparatus configuration for flowing water through the carbon bed. If the regenerant surfactant solution was applied directly to the dry carbon, severe foaming was observed. The foaming phenomena was previously observed by Blackburn (11). The foam caused severe channeling in the column, due to air trapped between the granulated carbon particles. Air was also trapped in the pores of the carbon. Therefore, the column was flushed with water, before the surfactant solution was introduced, until the air voids between the granulated carbon particles were no longer visually observed. The water flush step to remove the air pockets required three to four hours. The concentration of the desorbing solute in the effluent water was measured and used in calculation of the fractional removal of the solute.

Figure III.3 shows how the target organic solute was solubilized and removed from the carbon bed. A surfactant solution containing between $0.10 \mathrm{M}$ SDS and $0.30 \mathrm{M}$ SDS was pumped through the column at a flow rate of $2 \mathrm{~mL} / \mathrm{min}$ to $15 \mathrm{~mL} / \mathrm{min}$. The flow rate was set manually by a valve at the exit of the carbon bed and by manually adjusting a bypass valve integral to the pump head. The flow rate was measured every two hours at a minimum using a stop watch and graduated cylinder for five minute periods. Analysis of the effluent and a knowledge of the amount of 
solute originally loaded on the column permitted a calculation of fractional recovery at any point in the run.

Shown in Figure III.4 is the system configuration for the water flush that follows the micellar flood step. The water flush removes residual surfactant from the carbon bed. During the water flush step, 20 L of water were pumped through the bed at either $10 \mathrm{~mL} / \mathrm{min}$ or 20 $\mathrm{mL} / \mathrm{min}$.

Following the water flush, the bed was drained and the water bath temperature was elevated to $50^{\circ} \mathrm{C}$ to accelerate the drying process of the carbon bed. Compressed air was passed through a condenser (cold finger) to condense the carbon bed effluent water vapor as shown in Figure III.5. The dry air from the condenser was recycled back to the compressor and through the carbon bed. The drying was considered complete when condensate, upon visual inspection, ceased to appear in the condenser. The drying step took from 12 to 24 hours.

To avoid start-up effects due to using virgin carbon (11), three complete cycles of adsorption and regeneration were completed before quantitative experiments were conducted. In these pretreatment runs, 20 $\mathrm{mL}$ of organic were loaded on the carbon, then $14 \mathrm{~L}$ of $0.2 \mathrm{M}$ SDS regenerant solution and $20 \mathrm{~L}$ of water flushing solution were passed through the column. The previously described drying procedure was used. 


\subsection{SECR Liquid Phase Experiments}

The investigation of the regeneration of activated carbon used for liquid phase applications involved two types of experiments, dynamic column experiments and static equilibrium experiments.

\subsubsection{Dynamic Column Experiments}

Figures III.6 through III.9 show the different configurations of the experimental apparatus used for this investigation. The major equipment comprising the experimental apparatus are listed in Table III.1.

Figures III.6 and III.7 shows how the apparatus was configured for the adsorption of cetyl pyridinium chloride (CPC) onto the bed by continuously recycling the surfactant solution through the carbon bed for eight days at $30^{\circ} \mathrm{C}$. Figure III. 6 shows how the apparatus was configured when the vacuum was initiated on the carbon bed to prevent foaming of the surfactant when the carbon was wet by the surfactant solution. Very little foaming was observed as the surfactant solution filled the carbon bed over a 20 minute period. When the solution reached the top of the column, the vacuum was discontinued and the system was changed to the configuration shown in Figure III.7. The initial concentration of the surfactant solution and volume were known. The final concentration and final volume were measured. A mass balance was used to determine the level of adsorption on the bed and the residual surfactant in solution in 
the bed. If the surfactant solution was applied directly to the dry carbon bed, severe foaming was observed.

The water flush followed the surfactant adsorption step. Figure III.8 shows the configuration of the apparatu's when the beds were flushed with distilled and deionized water. Approximately one pore volume of water was flowed through the carbon bed to remove the residual surfactant solution in the bed. The temperature of one column was raised to $40^{\circ} \mathrm{C}$ and the other column to $50^{\circ} \mathrm{C}$ and the water flush continued at the new temperatures. Analysis of the effluent and a knowledge of the amount of surfactant originally loaded on the column permitted a calculation of fractional recovery at any point in the run. The water flush flow rate was held constant at $300 \mathrm{~mL}$ per hour and was checked often by measuring the volume of the water exiting the bed for a four minute time period. The flow rate did not vary by more than ten percent. Following the water flush, a tert-butylphenol solution was pumped through the bed using the apparatus configuration shown in Figure III.9. The flow rate was held constant at $1 \mathrm{~L} / \mathrm{hr}$ and was checked often. The concentration of the feed solution was $2667 \mu$ mole/L. The progress of the breakthrough curve was monitored by sampling the exit stream of the bed and analyzing the effluent samples using HPLC and a UV detector. The bed was maintained at $30^{\circ} \mathrm{C}$. 


\subsubsection{Static Equilibrium Experiments}

Kinetic studies to determine the period of time for adsorption equilibrium to occur were accomplished by equilibrating known concentrations of SDS and CPC solution with measured amounts of activated carbon. The studies were conducted at $30^{\circ} \mathrm{C}, 40^{\circ} \mathrm{C}$, and $50^{\circ} \mathrm{C}$ for 14 days. Wide mouth bottles with a volume of $100 \mathrm{~mL}$ were used to contact $25 \mathrm{~mL}$ of surfactant solution with 2.5 grams of carbon. The bottles were sealed using para film followed by a thin layer of styrofoam and the screw cap. The bottles with their contents were weighed. After equilibration, the bottles were weighed again to determine if any of the solution evaporated and if it did, a correction was made in the calculated adsorption. Samples were taken on a daily basis. The samples were analyzed for SDS and CPC using HPLC with a conductivity detector for SDS and a UV detector for CPC. Static isotherms at $30^{\circ} \mathrm{C}, 40^{\circ} \mathrm{C}$, and $50^{\circ} \mathrm{C}$ were measured for both SDS and CPC using the same techniques used for the kinetic studies, except that the samples were equilibrated for 10 days.

\subsection{Micellar-Enhanced Ultrafiltration}

A flow diagram of the ultrafiltration apparatus used for this study is shown in Figure III.10. The pump (P1) was a Burks stainless steel booster pump powered by a one fourth horsepower Franklin 120 volt motor. The temperature of the feed tank was held constant by pumping refrigerated coolant through a heat exchanger made of $1 / 4$ inch stainless 
steel tubing in a 4 inch diameter coil in the feed tank. A temperature probe and controller were used to control the coolant pump (P2) to provide the desired temperature in the feed tank. The feed pressure to the membrane was held constant at $60 \mathrm{psi}$. The retentate flow rate was held constant at $400 \mathrm{~mL}$ per minute. The apparatus start-up was done with the coarse adjustment pressure control valve (V1), a ball valve, completely open. The feed pressure to the membrane was then set at 60 psi. The retentate and permeate flow rate were measured by actuating two electromagnetic three way valves (V3 and V4) that diverted the retentate and permeate streams into collection beakers. The beakers with their solutions were weighed on a Ohaus triple beam balance and the weight of the empty beakers was subtracted to arrive at mass flow rates for the two streams. The electromagnetic sampling valves (V3 and V4) were controlled by a microprocessor controller that energized the valves for fifteen seconds. Using an iterative process of adjusting the retentate flow rate valve (V6) and the fine adjustment pressure control valve (V2) and measuring the mass flow rate of the retentate stream the 60 psi feed pressure and $400 \mathrm{~mL}$ retentate flow rate were established. The three-way system drain valve (V5) was used to drain permeate from the apparatus to increase the concentration of surfactant in the feed tank. The membrane, shown in Figure III.11, was a five square foot cellulose acetate spiral wound membrane manufactured by Spectrum Medical Products. In general, the permeate flow rate was $440 \mathrm{~mL}$ per minute for distilled and deionized water at a pressure of 60 psi gage on the retentate side of the membrane. 
The pilot plant was constructed to use one membrane module. Other membranes were allowed to remain in their pressure vessels when not used in the pilot plant. However, the unused membranes in their pressure vessels were connected to a manifold in the sink and house distilled water was continuously passed through the membranes. This treatment prevented the membranes from drying out and prevented bacteria and fungi from growing on the surface of the membrane.

The void volume of the pilot plant system was approximately 1000 $\mathrm{mL}$. Leaving the residual solution in the system can contaminate subsequent experiments if not removed. Prompt cleaning of the system was done at the end of each investigation to minimize the possibility of bacterial or fungal growth contaminating the system. The first step in cleaning the system was to remove as much of the residual solution from the feed tank as possible. The next step was to fill the feed tank with house distilled water and flush the system for five to ten minutes. The system was then drained and disassembled and tubing rinsed with house distilled water. The valves were rinsed by using a vacuum and sump to flow house distilled water through the valve. When the system was not in use, it was left disassembled and the membrane in a pressure vessel was purged with house distilled water.

A supplemental $20 \mathrm{~L}$ polypropylene tank, not shown in Figure III.10, was used to hold surfactant solution. The surfactant solution concentration in this supplemental tank was generally $0.080 \mathrm{M}$ to 0.120 M. Solution from the supplemental tank was added to the feed tank as the solution in the feed tank was concentrated by draining off permeate 
solution through valve V5. Using the supplemental tank allowed the initial feed concentration in the feed tank at the beginning of the experiment to be near the CMC for a given surfactant and by adding surfactant solution from the supplemental tank to reach a surfactant concentration approaching $0.6 \mathrm{M}$ by the end of the experiment.

The system reached equilibrium rapidly, normally within a few minutes, equilibration being indicated by a constant permeate flux, within a precision of one percent. Therefore, all the data presented are equilibrium values.

\subsection{Vacuum Stripping of Chlorinated Hydrocarbons from Micellar Solutions}

The stripping of trichloroethylene (TCE) and perchloroethylene (PCE) from surfactant solutions was studied from solutions containing a $0.2 \mathrm{M}$ Dowfax 8390 solution. The stripping of mixtures of these compounds was studied at $0.25 \mathrm{M}$ Dowfax 8390. The studies were performed at $22^{\circ} \mathrm{C}$ using a $20 \mathrm{~L}$ glass vessel maintained at 26 inches of mercury by a dry reciprocating pump powered by a one fourth horsepower, 120 volt motor. The mixture of chlorinated hydrocarbons and surfactant solution was introduced into the glass vacuum chamber by a spray nozzle at a rate of $114 \mathrm{~mL}$ per minute.

The above procedure was one of several studied and was chosen because it was the only method that did not produce significant amounts

of foam. The first unsuccessful apparatus was in $25 \mathrm{~mm}$ diameter and 
$1000 \mathrm{~mm}$ long column with a ring packing material. Significant foaming occurred at the surfactant solution inlet near the top of the stripping column. The foam traveled upward from the surfactant solution inlet to the top of the column where the vacuum port was located. Glass wool was packed above the solution inlet in an attempt to break the foam. When the foam reached the packing, the foam initially broke until the glass wool was saturated with surfactant solution from the broken foam. The foam continued to pass through the saturated glass wool, but the bubble size was smaller. The vacuum was discontinued when the foam entered the vacuum port at the top of the column.

A second unsuccessful apparatus used the column mentioned above with a spray nozzle and a gear pump to provide the driving force for atomizing the surfactant solution by the spray nozzle. The diameter of the column was too small for the spray pattern of the spray nozzle. The droplets of spray impinged on the walls of the column forming a film that foamed. The foaming film was eliminated by using a $20 \mathrm{~L}$ glass bottle with a diameter of approximately eighteen inches.

The concept behind atomizing the surfactant solution to prevent foaming is the creation of high surface area at the liquid-vapor interface. The large surface area and small diffusion paths in these small droplets allow rapid volatilization of solubilized volatile organic compounds (VOC's). The spray npzzle was obtained from a commercial hand operated Easy Off ${ }^{\circledR}$ oven cleaner dispenser and modified for this application. There are ultrasonic spray nozzles that are much more 
efficient and operate at much lower pressures than the 100 psig that was used for the stripping experiments.

The stripping experiments were accomplished using a batch process. Shown in Figure III.12 is the configuration of the experimental apparatus used for the stripping studies. The first step was to close all valves and evacuate the $20 \mathrm{~L}$ glass vessel. The $20 \mathrm{~L}$ glass vessel was wrapped with fiberglass reinforced tape as a safety precaution in the event the glass vessel imploded. When the pressure in the glass vessel reached 26 inches of mercury as measured by G1, the gear pump P2 was activated and valve V1 was opened. Approximately $3.5 \mathrm{~L}$ of surfactant solution with solubilized chlorinated hydrocarbons was sprayed into the $20 \mathrm{~L}$ glass vessel. When the pressure, as registered by P2, dropped to 50 psig, due to the suction of air into the pump when the feed solution container was emptied, the gear pump was deactivated and the vacuum was released by opening valve V2. The solution at the bottom of the $20 \mathrm{~L}$ glass vessel was sampled when the pressure in the glass vessel was equalized with the atmospheric pressure. The remaining solution was returned to the feed solution container and the experiment was started again. The samples were analyzed using a Tekmar 2000, Varian GC, and Hall detector using the same method as was used for MEUF samples of chlorinated hydrocarbon surfactant mixtures except that the chlorinated hydrocarbon calibration curves were generated using TCE standards in a $0.2 \mathrm{M}$ surfactant solution for the single component system and $0.25 \mathrm{M}$ surfactant solution for mixtures of chlorinated hydrocarbons. 


\subsection{Vapor-Liquid Equilibrium Studies}

Vapor-liquid equilibrium studies of chlorinated hydrocarbon compounds in aqueous solutions and in $0.05 \mathrm{M}$ surfactant solutions were done by analyzing the vapor in the headspace above the liquid phase using gas chromatography. Samples of $20 \mathrm{~mL}$ of aqueous solution and surfactant solution with known concentrations of chlorinated hydrocarbon in $40 \mathrm{~mL}$ EPA standard water analysis vials were allowed to equilibrate in a temperature controlled bath for 24 hours. After the samples had equilibrated, $0.5 \mathrm{~mL}$ was drawn from the headspace above the liquid and evaluated using a Perkin-Elmer gas chromatography.

\subsection{Materials}

Shown in Figure III.13 through III.15 are the structures of the compounds that were investigated. Shown in Table III.2 is general information such as critical micelle concentration (CMC), formula weight, purity, vendor and catalog number for the surfactants investigated. Table III.3 lists similar information for the target organic solutes.

\subsubsection{Sodium Dodecyl Sulfate}

Two different SDS surfactants were used for the SECR and MEUF investigations. There was a considerable cost difference between the Aldrich SDS (\$8.00/500g) compared to the Fisher SDS $(\$ 25 / 25 \mathrm{~g})$. The 
amount of surfactant solution required for each SECR experiment was a minimum of $14 \mathrm{~L}$ of $0.2 \mathrm{M}$ SDS, while a single MEUF experiment required a minimum of $3 \mathrm{~L}$ of $0.6 \mathrm{M}$ SDS. Considering the concentration and volume of surfactant solution that was required, a reasonable compromise was accepted. The Fisher SDS was used when making standards and the Aldrich SDS was used for the actual experiments. The Aldrich SDS was composed of three surfactants SDS, sodium tetradecyl sulfate (STS), and sodium hexadecyl sulfate (SHS). When analyzed using HPLC/conductivity (explained in the analytical section), there were three distinct peaks, one for each surfactant, and a significant salt peak. The salt was removed from the Aldrich SDS by ultrafiltration of the surfactant solution. The procedure consisted of making $25 \mathrm{~L}$ of $0.04 \mathrm{mM}$ SDS solution and utrafiltering the solution until the retentate volume was $5 \mathrm{~L}$ and the permeate volume was $20 \mathrm{~L}$. The permeate was discarded. The $5 \mathrm{~L}$ of retentate was diluted with distilled and deionized water to make $25 \mathrm{~L}$ and ultrafiltered again. The process was repeated a total of 5 times. Material balance calculations indicate that $99.7 \%$ of the unbound salts are removed from the surfactant solution and discarded in the permeate solution. HPLC analysis showed very small amounts of salt for the MEUF processed Aldrich SDS relative to the unprocessed Aldrich SDS. The Fisher SDS was used as the standard because it was a well characterized product, while the Aldrich SDS was an industrial grade surfactant. 


\subsubsection{Dowfax 8390 and Dowfax 3B2}

The Dowfax series of surfactants, when received from Dow Chemical Co., contained as much as two percent methylene chloride and four percent salts. The salts were removed by using the same process used for desalting Aldrich SDS as described in section 3.4.1. The methylene chloride was removed by boiling a $0.2 \mathrm{M}$ surfactant solution at an absolute pressure of 17 inches of mercury for 35 minutes. The temperature of the solution ranged from $80^{\circ} \mathrm{C}$ to $90^{\circ} \mathrm{C}$ with the most vigorous boiling occurring at the beginning of the process. This was accomplished in a $12 \mathrm{~L}$ pyrex round bottom flask filled with $3 \mathrm{~L}$ of surfactant concentrate and $3 \mathrm{~L}$ of distilled and deionized water and using an electric heating mantle. Generally the solution was allowed to cool overnight. Samples of the surfactant solution were evaluated using a Tekmar 2000, Varian GC and a Hall detector. No methylene chloride was detected. Dow Chemical Co. now offers DOWFAX 8390 free of methylene chloride and with 1 percent salts.

\subsubsection{Other Surfactants}

Other surfactants investigated were CPC, DNP-18 and STEDBAC. All of these surfactants were used without further refining or processing except for storage of ionic surfactants under a vacuum with a desiccant. 


\subsubsection{Target Organics Investigated}

All of the target organics listed in Table III.3 were used as received except for the TBP. The TBP was 99 percent pure as received but had a definite pale yellow color. A purification technique used by Blackburn (27) was used for removing the yellow color and insoluble material from the TBP used in the investigations described in this work. The latest shipwent of TBP used here had no yellow hue and no insoluble materials but still listed the purity as 99 percent.

\subsubsection{Target Metals}

The target metals listed in Table III.4 were used as received except for storage in evacuated containers with desiccant with the exception of cupric chloride. Cupric chloride is extremely hygroscopic and required drying in an oven to drive off all the waters of hydration. The cupric chloride usually had blue colored water droplets adhering to the walls of the bottle and the crystals were blue and clumpy as received and stored on the shelf in the lab. By drying the crystals at $60^{\circ} \mathrm{C}$ in an oven overnight, the crystals turned brown and were no longer clumpy. A 1000 ppm copper solution was made using the oven dried cupric chloride and compared against a commercially available $1000 \mathrm{ppm}$ copper solution standard available from Fisher using flame atomic adsorption to confirm that the brown crystals were cupric chloride without any waters of 
hydration. After drying, the cupric chloride was stored in an evacuated container with desiccant.

\subsection{Analytical Methods}

Three analytical techniques were used to evaluate samples to determine concentrations of surfactants and target compounds. HPLC was used to determine all surfactant, TBP and toluene concentrations. Atomic adsorption spectroscopy was used to determine all target metal concentrations. Gas chromatography was used to determine all halogenated hydrocarbon and amyl acetate concentrations.

\subsubsection{HPLC Analytical Methods}

Shown in Table III.5 is a list of the equipment comprising the HPLC system used to determine concentrations of SDS, DOWFAX 8390 and DOWFAX 3B2. Shown in Table III.6 are the solvents and method of HPLC operation for each compound.

Two modes of operation, isocratic and solvent switching, were used for the analysis of ionic compounds. The isocratic mode of operation consisted of using a single mobile phase ( solvent) for the entire analysis. The solvent switching mode uses different mobile phases during the analysis. The isocratic mode of operation experienced fewer incidents of pump cavitation when compared to step function solvent switching. 
TABLE III.5

HPLC Equipment Used to Evaluate Samples for Ionic Compounds

\begin{tabular}{|c|c|}
\hline PUMP & Perkin-Elmer Series 10 \\
\hline PUMP & Tracor Model 951A \\
\hline DETECTOR & Varian Conductivity \\
\hline INTEGRATOR & Varian 4270 \\
\hline INJECTOR & Rheodyne 7125 \\
\hline SOLVENT SWITCHING & Angar Scientific Model 368140Q \\
SOLENOID AND VALVE & 20 psi, 115V \\
\hline COLUMN PACKING & Whatman Parisil-10 ODS-3 \\
\hline SYRINGE & $\begin{array}{c}1.0 \text { mL Becton-Dickson glass } \\
\text { tuberculin luer tipped }\end{array}$ \\
\hline
\end{tabular}

During step function solvent switching, an abrupt change from one mobile phase to an alternate mobile phase occurred. The mixing of methanol and water was exothermic causing an elevation of the solution temperature. The temperature increase elevated the vapor pressure of the mixture allowing cavitation of the solvent on the suction side of the positive displacement HPLC pump. The problem was less severe with the Tracor pump due to the double piston design. The intake stroke was equal in duration to the pump stroke. The Perkin-Elmer was fabricated with one pump head and had a very short intake stroke with a pump stroke estimated to comprise $80 \%$ of the cycle, thus generating a greater pressure drop across the intake valve, making it easier for cavitation to occur. The short duration intake stroke was necessary in order to resume the pump stroke in a effort to minimize pressure surges and therefore flow rate 
surges. The Perkin-Elmer pump was adequate for isocratic solvent systems.

TABLE III. 6

Solvents and Mode of Operation of HPLC for Analysis of Ionic Compounds

\begin{tabular}{|c|c|c|}
\hline COMPOUND & MOBILE PHASE & MODE \\
\hline DOWFAX 8390 & $70 \%$ Methanol & Isocratic \\
& $30 \%$ Water & \\
\hline DOWFAX 3B2 & $35 \%$ Methanol & Isocratic \\
& $65 \%$ Water & \\
\hline SDS (Fisher) & $65 \%$ Methanol & Isocratic \\
SDS (Aldrich) & $35 \%$ Water & \\
\hline & Solvent \#1 & \\
SDS (Fisher) & $10 \%$ Methanol & Step Function \\
SDS (Aldrich) & $90 \%$ Methanol & Solvent Switching \\
& Solvent \#2 & \\
& $100 \%$ Methanol & \\
& &
\end{tabular}

One of the advantages of step function solvent switching is the compression of similar compounds into one peak. This was very useful for the Aldrich SDS which is actually a homologous mixture of surfactants. The use of solvent \#1 allowed the removal of salts from the column while leaving the mixture of surfactants hydrophobically bound to the $\mathrm{C}_{18}$ groups bonded to the reverse phase silica packing material. The introduction of solvent \#2 into the packed column allowed the desorption of the surfactant compounds simultaneously, thus giving one peak. The 
calibration curve used the Fisher SDS with the solvent switching technique. The conductivity detector did not distinguish between the different surfactants but did measure the conductivity of the solution which was dependent on the concentration of sulfate ions in solution. The use of the Fisher SDS calibration curve allowed the accurate determination of the ionized sulfate concentration and therefore, an accurate determination of the total sulfate concentration of the Aldrich surfactant mixture.

Some of the other advantages of step function solvent switching were the "cleaning" action of using a very polar solvent and a relatively nonpolar solvent after each injection and the relatively low operating pressures. Solvent \#1 was $90 \%$ water and was very effective at eluting salts from the column. Solvent \#2 was $100 \%$ methanol and was very effective at eluting organic compounds from the column. The extreme range for the two solvent systems insured a more effective elution of salts and organics than operating in the isocratic mode. Also, the operating pressure was higher for the isocratic operation than for the step function solvent switching mode. A $50 \%$ water / $50 \%$ methanol mixture had the highest system pressure, probably because the viscosity of the mixture was greater than for the individual pure compounds. Considering the two solvents used for the solvent switching were either pure methanol or $90 \%$ water, it was no surprise that the system pressure was generally less except during the actual solvent switch. The pressure for pure water or methanol at a $3 \mathrm{~mL} / \mathrm{min}$ flow rate was 700 to 900 psig where as the pressure of a 50-50 mix of methanol and water at the same flow rate was 
3500 psig. In general, the solvent switching allowed operating the system at elevated flow rates allowing shorter analysis periods.

The Varian 4720 was used as a data acquisition device and for plotting. Usirig a ruler graduated in $1 \mathrm{~mm}$ segments to measure peak heights gave the most repeatable results. The Varian 4720 was also used for the timing and control for the step function solvent switching. Copies of programs for isocratic and step function solvent switching are included in the appendix.

Shown in Table III.7 is a list of HPLC equipment used to evaluate surfactant solutions containing Dowfax 3B2, Dowfax 8390, cetyl pyridinium chloride, stearyl dimethyl benzyl ammonium chloride and polyoxyethylene dinonyl phenol. Shown in Table III.8 is a list of the composition of the mobile phase and the mode of operation for each compound.

\section{TABLE III.7}

HPLC Equipment Used to Evaluate Surfactants and Target Organics with a Chromophore

\begin{tabular}{|c|c|}
\hline PUMP & Perkin-Elmer Series 10 \\
\hline PUMP & Tracor Model 951A \\
\hline DETECTOR & Tracor Model \\
\hline INTEGRATOR & Varian 4270 \\
\hline INJECTOR & Rheodyne 7125 \\
\hline SOLVENT SWITCHING & Angar Scientific Model 368140Q \\
SOLENOID AND VALVE & 20 psig, 115V \\
\hline COLUMN PACKING & Whatman Parisil-10 ODS-3 \\
\hline SYRINGE & $\begin{array}{c}1.0 \text { mL Becton-Dickson glass } \\
\text { tuberculin luer tipped }\end{array}$ \\
\hline
\end{tabular}


TABLE III.8

Solvents and Mode of Operation of HPLC for Analysis of

Surfactants and Target Organics with Chromophore.

\begin{tabular}{|c|c|c|}
\hline COMPOUND & MOBILE PHASE & MODE \\
\hline DOWFAX 8390 & $70 \%$ Methanol & Isocratic \\
& $30 \%$ Water & wavelength = 220 nm \\
\hline DOWFAX 3B2 & $35 \%$ Methanol & Isocratic \\
& $65 \%$ Water & wavelength = 220 nm \\
\hline \multirow{2}{*}{ CPC } & $95 \%$ Methanol & Isocratic \\
& $5 \%$ Water & wavelength = 260 nm \\
& $2 \mathrm{~g} / \mathrm{L} \mathrm{CaCl} 2$ & \\
\hline DNP-18 & $75 \%$ Methanol & Isocratic \\
& $25 \%$ Water & wavelength = 220 nm \\
\hline \multirow{3}{*}{ STEDBAC } & $95 \%$ Methanol & Isocratic \\
& $5 \%$ Water & wavelength $=220 \mathrm{~nm}$ \\
\hline TBP & 2 g/L CaCl 2 & Isocratic/ \\
& $60 \%$ Methanol & Solvent Switching \\
\hline Toluene & $40 \%$ Water & Isocratic \\
& $60 \%$ Methanol & wavelength = 220 nm \\
\hline
\end{tabular}

The DOWFAX surfactants can be analyzed using either a conductivity or ultraviolet (UV) detector. ,It was generally easier to use the UV detector because the conductivity detector was very sensitive to any extraneous salts that may have contaminated the HPLC system from previous solvents with added salts such as the CPC solvent. All of the investigations using DOWFAX and DNP-18 surfactants allowed the straightforward analysis since they were not in solutions with target 
compounds that had chromophores. Therefore, isocratic operation was the best mode of operation.

The two cationic surfactants, CPC and STEDBAC, presented more of a challenge. Silica has a net negative surface charge at a $\mathrm{pH}$ above approximately 2. Unlike anionic and nonionic surfactants which only have a hydrophobic interaction with the reverse phase silica packing, the cationic surfactants also have an electrostatic attraction with the negative charge on the surface. Initially the use of Whatman Partisil ODS-3 solved the problem by neutralizing the negative charge by adsorbing an oppositely charged compound on the silica surface. However, as the packing material aged (only a few weeks) the neutralizing compound desorbed leaving the surface with a net negative charge. The addition of calcium chloride to the mobile phase increased the ionic strength, compressing the electrical double layer of the charged surface and surfactant head group, thus decreasing the interaction of the surfactant head group with the silica surface. The concentration of $2 \mathrm{~g} / \mathrm{L}$ of calcium chloride is a starting value depending on the age of the packing material. The retention time wsa affected by both the percentage of methanol and the amount of added salt. In general the width of the peak was very sensitive to the amount of added salt and the retention time is more sensitive to the methanol concentration. If a peak has excessive tailing and added salt does not affect the tailing, it has been found that the addition of $2 \mathrm{~mL}$ of phosphoric acid to a liter of solvent reduces the tailing significantly. Considering the variability of the charge on the surface of the silica, the values in Table III. 8 will allow the elution of the surfactant 
out of the column but some adjustment of the system with additional salt and acid to optimize the analysis may be necessary. It takes several days for calcium chloride to dissolves into solution in methanol at room temperature, so the calcium chloride should be dissolved in a small amount of water and then added to the salt solution to the methanol.

The analysis of solutions for CPC often had another compound in solution such as TBP which also had a chromophore. Considering the high methanol percentage of the CPC mobile phase solvent, the target organic compound went through the column with minimal retention on the packing and eluted from the column well before the CPC peak, making the analysis straightforward for the CPC. The target organic was eluted as part of the solvent peak and was not measurable. Therefore, the sample was reevaluated using a different mobile phase to measure the target organic concentration. The mobile phase for TBP is listed in Table III.8 and is significantly different from the mobile phase used for CPC. The mobile phase for TBP did not elute the CPC from the column and therefore the analysis for TBP was not complicated by the presence of CPC. However, the CPC continued to reside on the column unless it was removed after each sample injection. CPC removal from the bed was accomplished by a step function solvent switch to the CPC solvent for 3 minutes. If the CPC was allowed to reside on the column, eventually the column pressure increased, often requiring lower flow rates to keep the pump pressure less than $3,000 \mathrm{psig}$. Changes in retention time and peak shape were also observed for the TBP peaks if the CPC was allowed to build up on the column. 
Other solution mixtures that required analysis were SDS/TBP and SDS/Toluene. These were relatively easy to analyze because the SDS was analyzed using the conductivity detector while toluene and TBP were analyzed on the UV detector. The UV detector was blind to the SDS and the conductivity detector was blind to toluene and TBP. The only disadvantage was the requirement to evaluate each solution twice, one time for each detector.

In the early days of these investigations the solvents were habitually degassed. However, it became apparent that by mixing the solvents a day or two in advance, the solutions cooled to room temperature and pump cavitation was not a problem even if the solvents were not degassed. Therefore, almost all of the solvents were made at least a day in advance and were not degassed.

\subsubsection{Gas Chromatography Analysis}

MEUF studies of mixtures of surfactant and chlorocarbon were performed using surfactant feed concentrations of $0.05 \mathrm{M}$. The $0.05 \mathrm{M}$ concentration was selected so the data generated by the liquid-vapor studies could be directly compared with the MEUF data. The chlorinated organic concentrations in samples generated by the MEUF apparatus were evaluated using Tekmar 2000 purge and trap in conjunction with a Varian 3000 gas chromatograph (GC) with a 60 meter Supelco Vocol megabor column and a Tracor 1000 Hall Detector. The carrier and purge gas was helium and the reactor gas was hydrogen. The purge and trap 
apparatus was operated in a headspace analysis mode due to the presence of foaming agents (surfactants) in the samples. The headspace analysis was performed by purging the headspace above $5 \mathrm{~mL}$ of sample solution thermostatted at $40^{\circ} \mathrm{C}$ for 12 minutes. Two calibration curves were generated since the vapor pressure of chlorinated hydrocarbon is directly related to the presence of micelles. The permeate samples were compared against calibration curves generated by chlorinated organic standards with surfactant concentrations below the CMC. Retentate samples were compared against calibration curves generated by chlorinated organic standards with surfactant concentrations of $0.05 \mathrm{M}$ surfactant.

The headspace of the equilibrium liquid-vapor surfactant/chlorinated organic solubilization samples was evaluated using a Perkin-Elmer gas chromatograph. A 3 foot long and 1/8 inch outside diameter column packed with a porous polymer based on 2,6-diphenyl-pphenylene oxide with a mesh range of $80 / 100$ was used at column temperatures of $180^{\circ} \mathrm{C}$, injection temperature of $190^{\circ} \mathrm{C}$, and a detector temperature of $200^{\circ} \mathrm{C}$. The carrier gas was helium and the detector gas was hydrogen.

\subsubsection{Flame Atomic Adsorption}

Analysis of chromate and copper were carried out using a Varian Spectra AA-20 variable wavelength atomic absorption spectrophotometer. The gases used were house compressed air and acetylene. Chromate analysis used a Varian chromium cathode lamp with a lamp current of 7 $\mathrm{mA}$, a wavelength of 357.9 and a slit width of $0.2 \mathrm{~nm}$. Copper analysis 
used a Varian copper cathode lamp with a lamp current of $4 \mathrm{~mA}$, a wavelength of $324.8 \mathrm{~nm}$ and a slit width of $0.5 \mathrm{~nm}$. The standards used.for calibration curves were composed of mixtures of the surfactant and the target metal ion. The molar ratio of the surfactant to target metal ion was set at 10 to 1 . 
TABLE III.2

General Information About Surfactants Investigated

\begin{tabular}{|c|c|c|c|c|}
\hline SURFACTANT & $\begin{array}{l}\text { CMC } \\
{[\mathrm{mM}]}\end{array}$ & $\begin{array}{l}\text { FORMULA } \\
\text { WEIGHT }\end{array}$ & QUALITY & VENDOR \\
\hline DODECYL SODIUM SULFATE & 8.20 & 288.38 & $99.7 \%$ & $\begin{array}{c}\text { FISHER } \\
\text { CAT } * 0-2674\end{array}$ \\
\hline CETYL PYRIDINIUM CHLORIDE & 0.880 & 358 & $99.9 \%$ & HEXCEL \\
\hline DOWFAX 3B2 & 20 & $\begin{array}{c}540 \\
\text { (ave) }\end{array}$ & $\begin{array}{l}\text { 41\% BY WEIGHT, AS MUCH AS } 2 \% \\
\text { METHYLENE CHLORIDE, AS MUCH AS } \\
\text { 5\% SALT }\end{array}$ & DOW \\
\hline DOWFAX 8390 & 0.5 & $\begin{array}{l}640 \\
\text { (ave) }\end{array}$ & $\begin{array}{l}\text { 41\% BY WEIGHT, AS MUCH AS } 2 \% \\
\text { METHYLENE CHLORIDE, AS MUCH AS } \\
5 \% \text { SALT }\end{array}$ & DOW \\
\hline
\end{tabular}




\section{TABLE III.3}

General Information About Target Organics Investigated

\begin{tabular}{|c|c|c|c|}
\hline COMPOUND & $\begin{array}{l}\text { FORMULA } \\
\text { WEIGHT }\end{array}$ & QUALITY & VENDOR \\
\hline 4-tert-BUTYLPHENOL & 150.22 & $99 \%$ & $\begin{array}{c}\text { ALDRICH } \\
\text { CAT } \# \text { B9,990-1 }\end{array}$ \\
\hline TOLUENE & 92.14 & ANALYTICAL REAGENT & $\begin{array}{c}\text { MALLINCKRODT } \\
\text { CAT } * 8608\end{array}$ \\
\hline 1,1,1-TRICHLOROETHANE & 133.41 & $99 \%$ & $\begin{array}{c}\text { ALDRICH } \\
\text { CAT } * 29.899-9\end{array}$ \\
\hline TETRACHLOROETHYLENE & 165.83 & $99.9 \%$ & $\begin{array}{c}\text { ALDRICH } \\
\text { CAT } * 27,039-3\end{array}$ \\
\hline AMYL ACETATE & 130.19 & "PURIFIED" & $\begin{array}{c}\text { MALLNCKRODT } \\
\text { CAT } * 3564\end{array}$ \\
\hline DICHLOROMETHANE & 84.93 & $99.9 \%$ & $\begin{array}{c}\text { ALDRICH } \\
\text { CAT }: 27,056-3\end{array}$ \\
\hline TRICHLOROETHYLENE & 131.39 & ANALYTICAL REAGENT & $\begin{array}{c}\text { FISHER } \\
\text { CAT : T341-500 }\end{array}$ \\
\hline
\end{tabular}


TABLE III.4

General Information About Target Metals Investigated

\begin{tabular}{|c|c|c|c|}
\hline COMPOUND & $\begin{array}{c}\text { FORMULA } \\
\text { WEIGHT }\end{array}$ & QUALITY & VENDOR \\
\hline Cupric Chloride Dihydrate & 170.48 & Reagent Grade & $\begin{array}{c}\text { Fisher } \\
\text { Cat \# C-455 }\end{array}$ \\
\hline Sodium Chromate & 161.97 & Reagent Grade & $\begin{array}{c}\text { Fisher } \\
\text { Cat \# S-272 }\end{array}$ \\
\hline
\end{tabular}




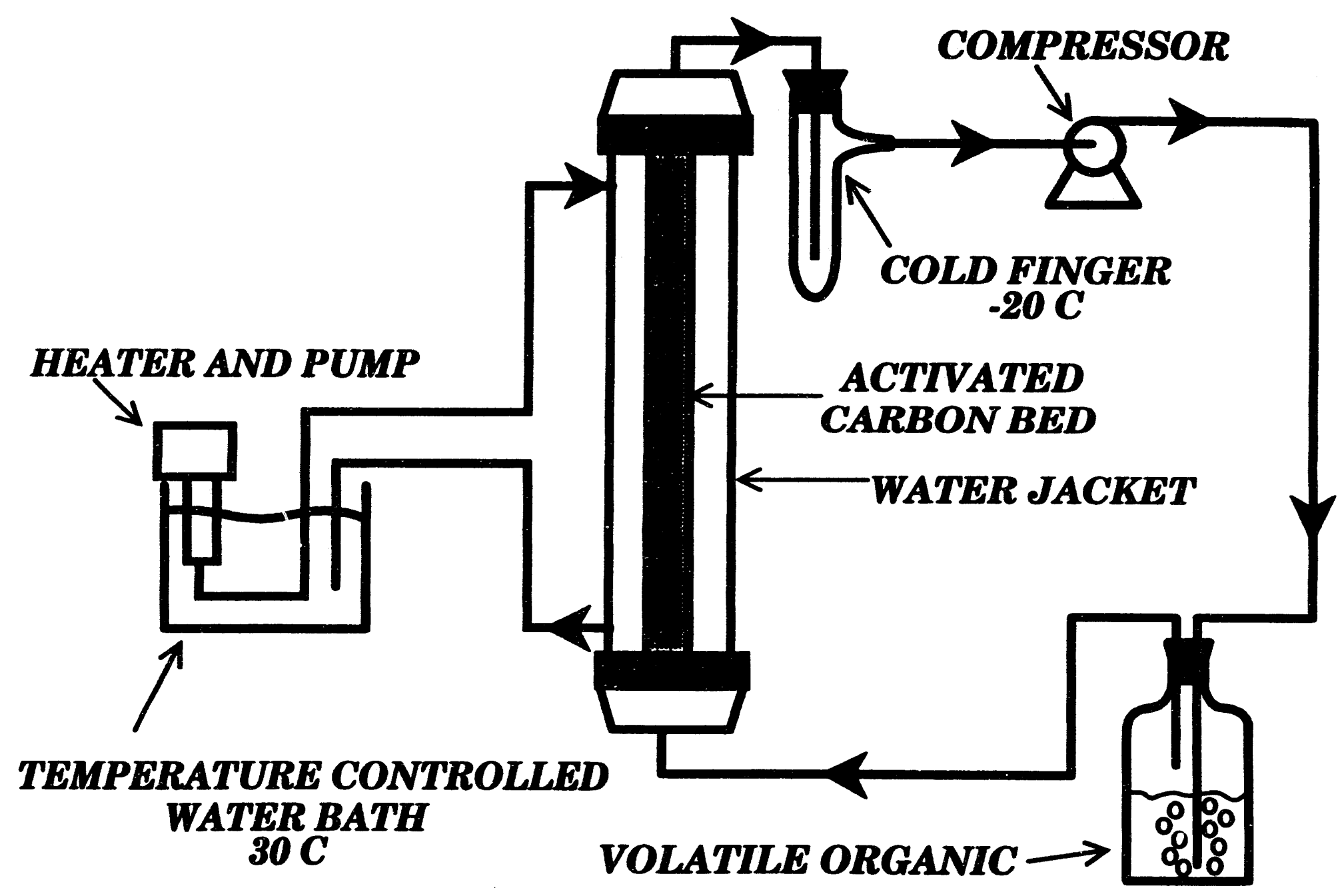

FIGURE III.1 Apparatus for Adsorption of the Target Organic Solute on Activated Carbon. 


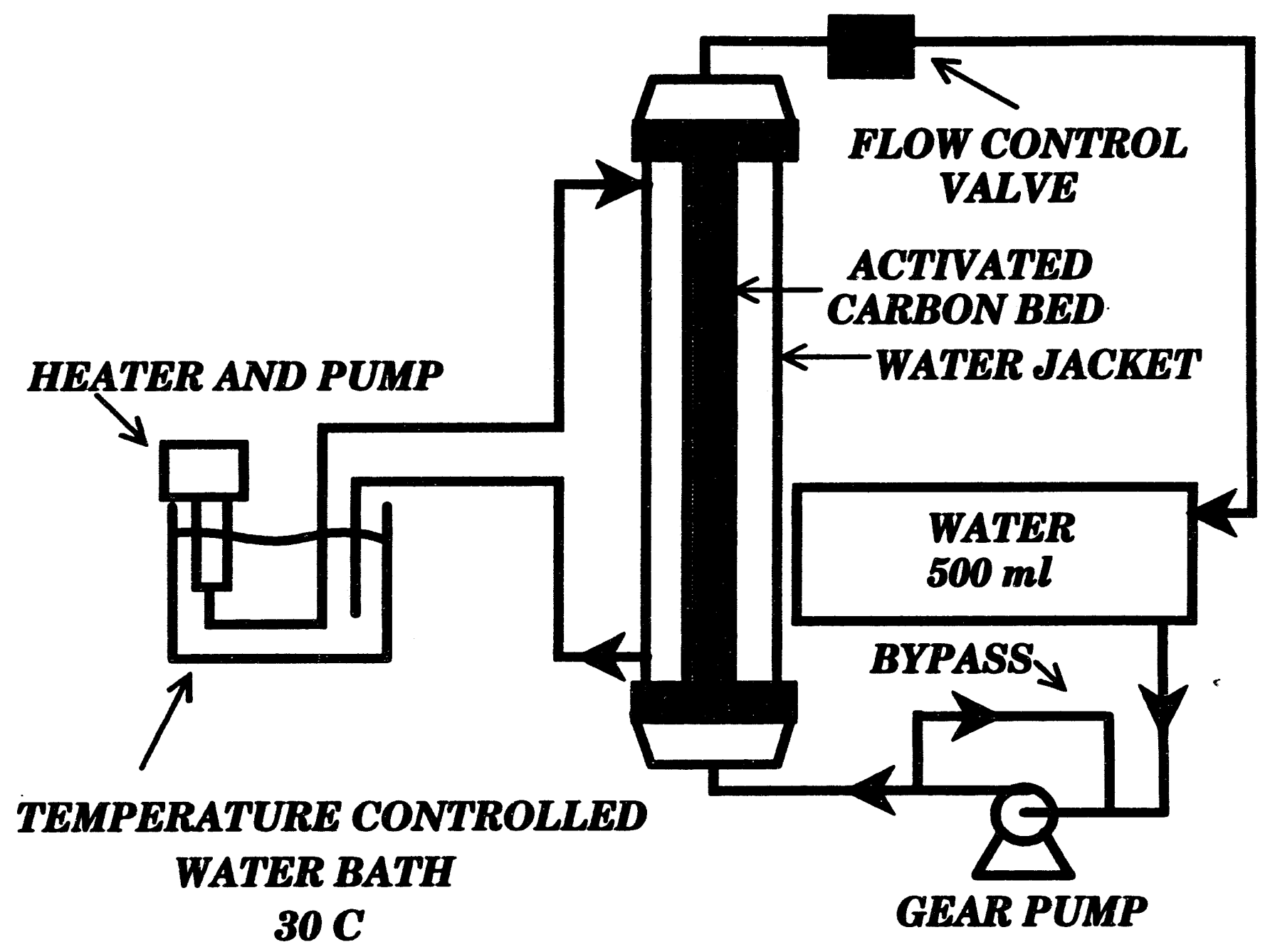

FIGURE III.2 Apparatus for Water Flush to Wet Activated Carbon 


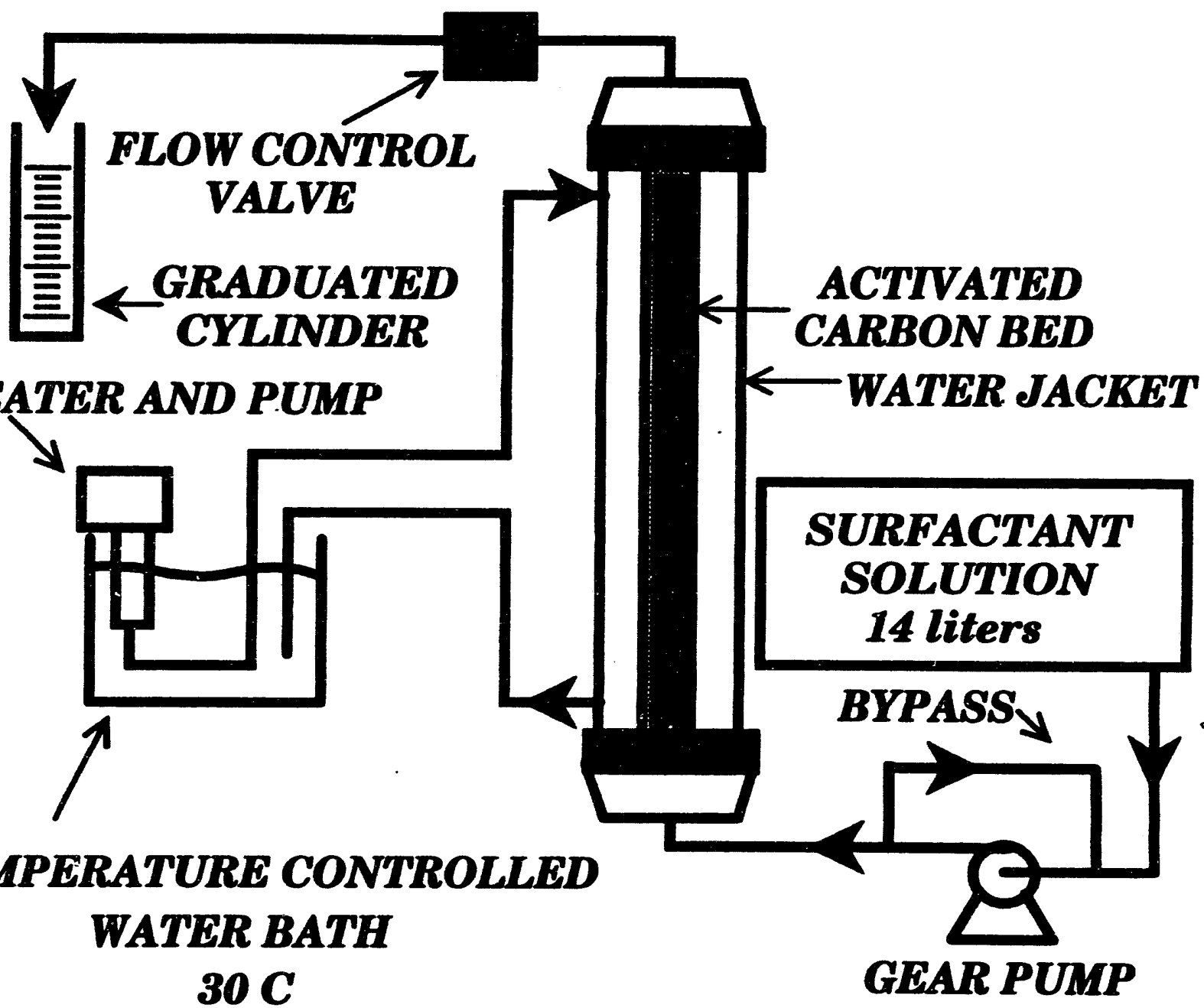

FIGURE III.3 Apparatus for Micellar Flood to Solubilize and Remove the Target Organic in SECR 


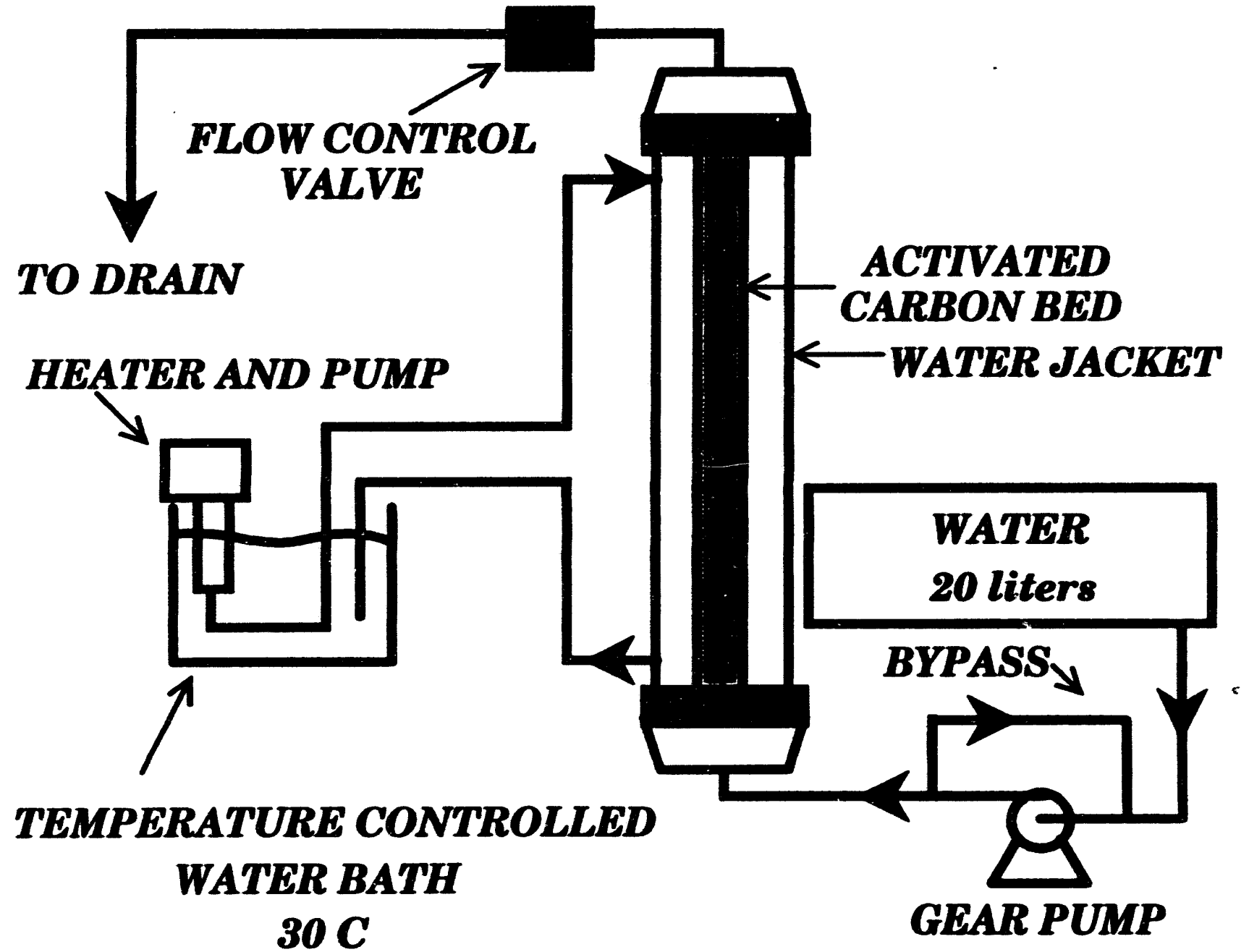

FIGURE III.4 Apparatus for Water Flush to Remove Residual Surfactant in SECR 


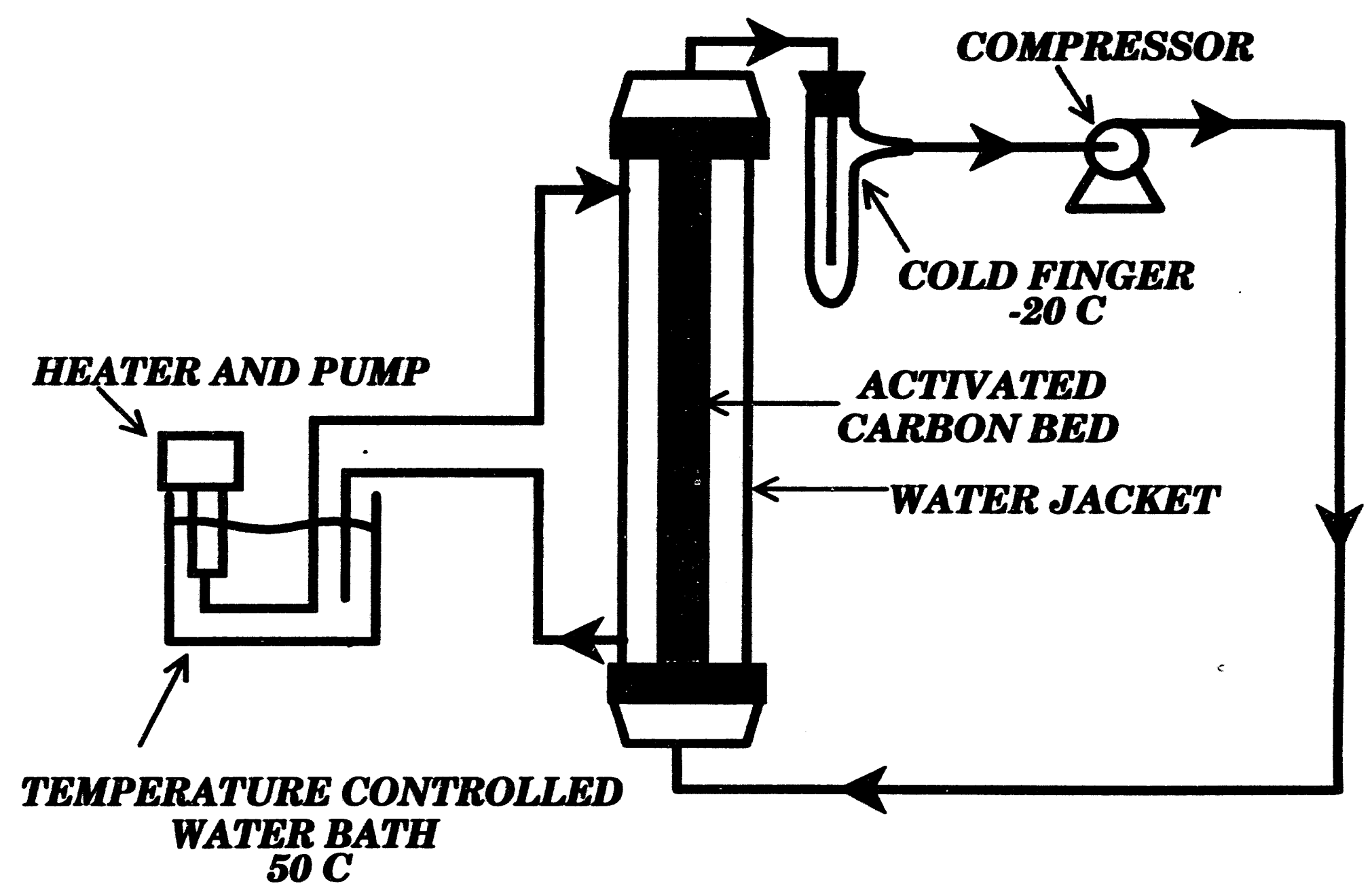

FIGURE III.5 Apparatus for Drying of the Activated Carbon Bed 


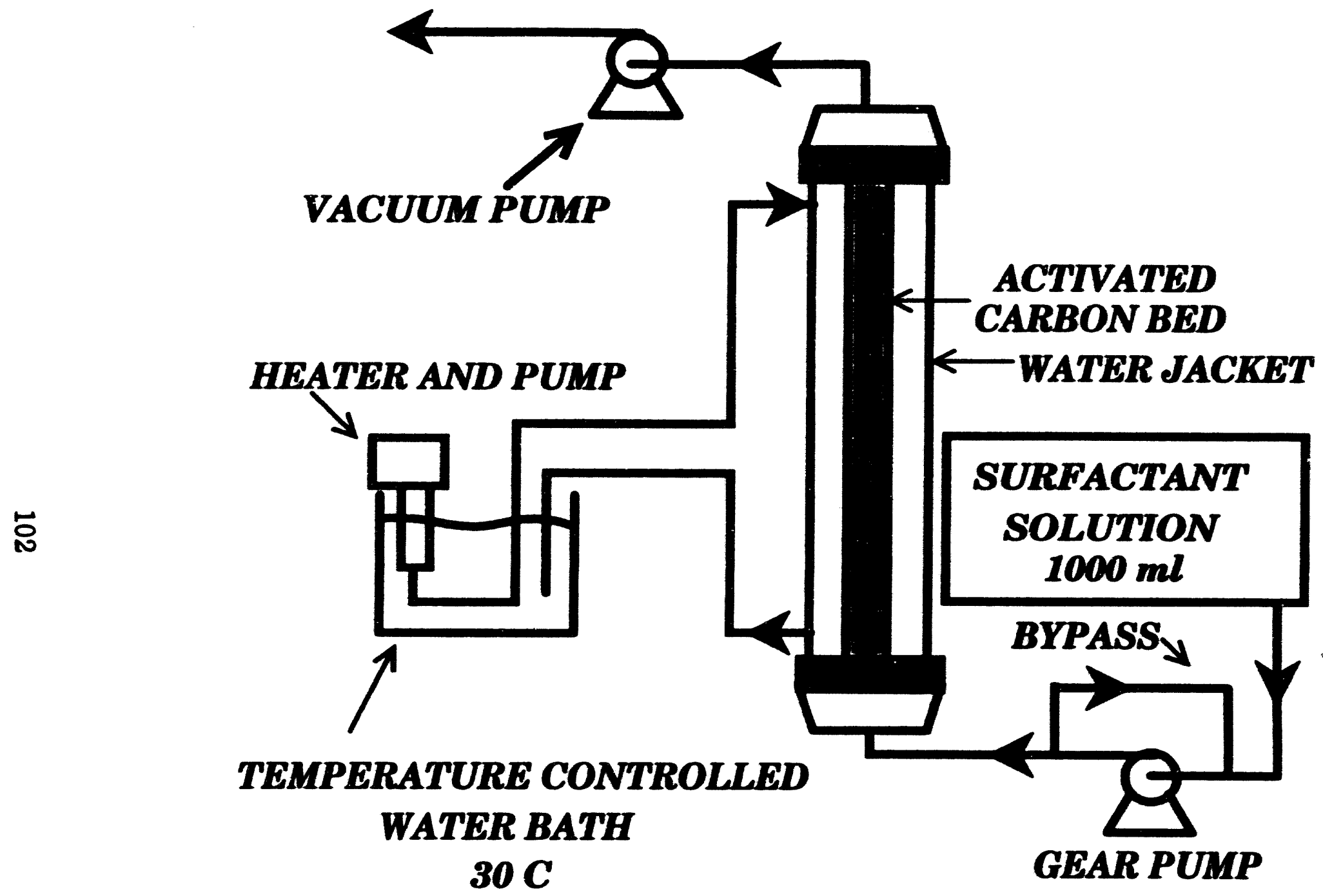

FIGURE III.6 Apparatus for Vacuum Application to the Carbon Bed for the Prevention of Foam Formation upon Introduction of the Surfactant Solution into the Bed 


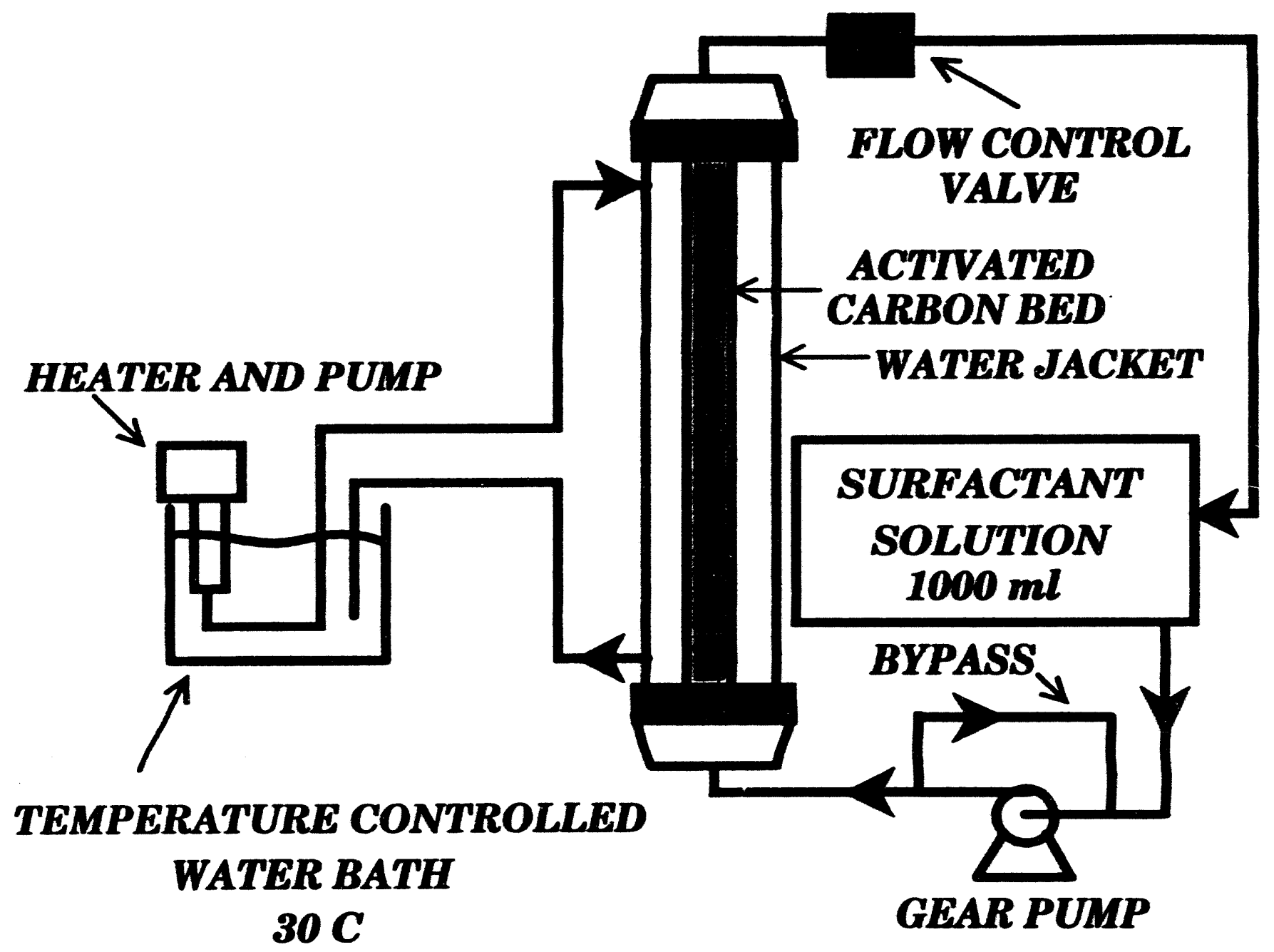

FIGURE III.7 Apparatus for the Adsorption of Cetyl Pyridinium Chloride onto the Carbon 


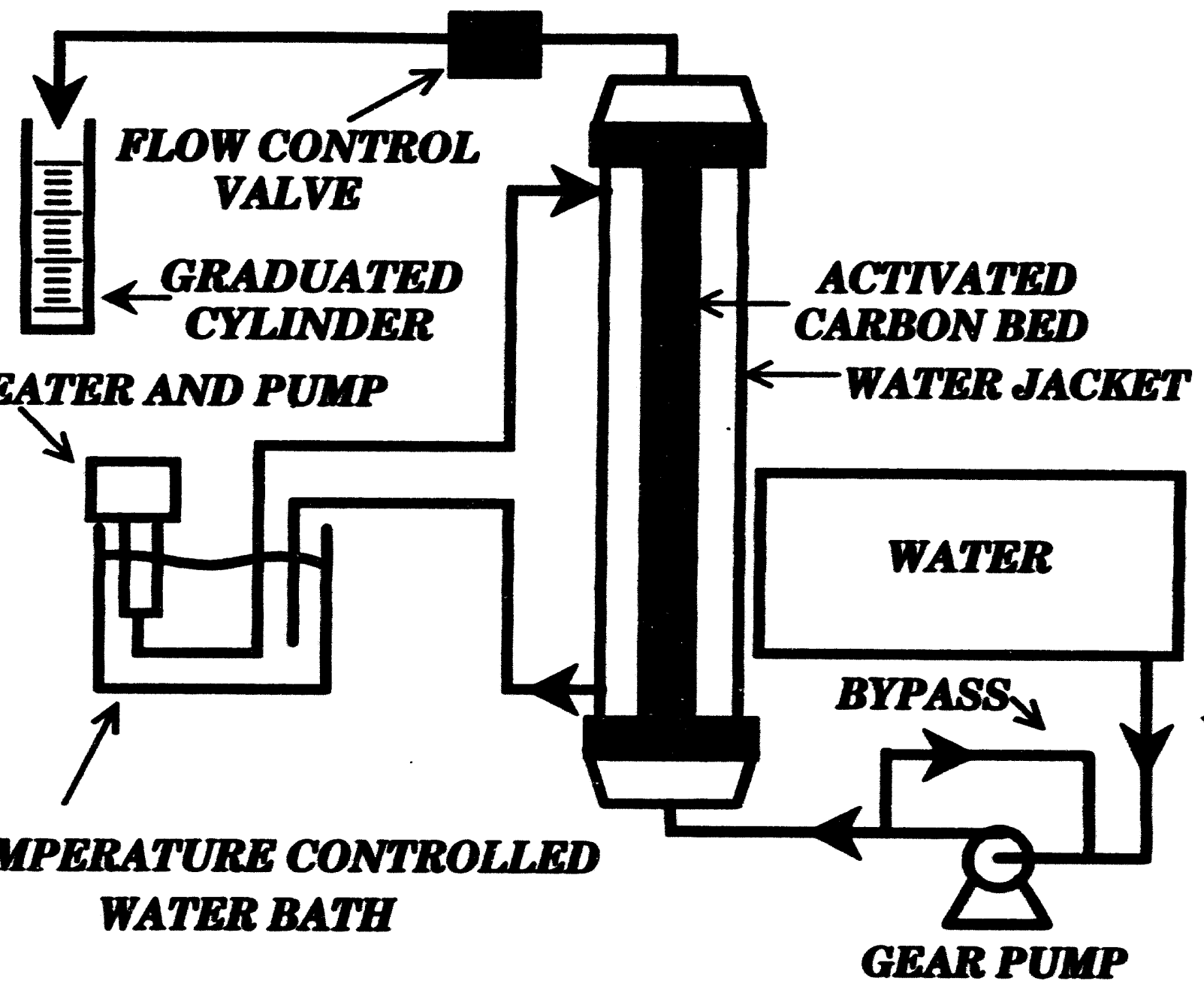

FIGURE III.8 Apparatus for Water Flush of the Column Void Volumn and Desorption of Surfactant from the Carbon 


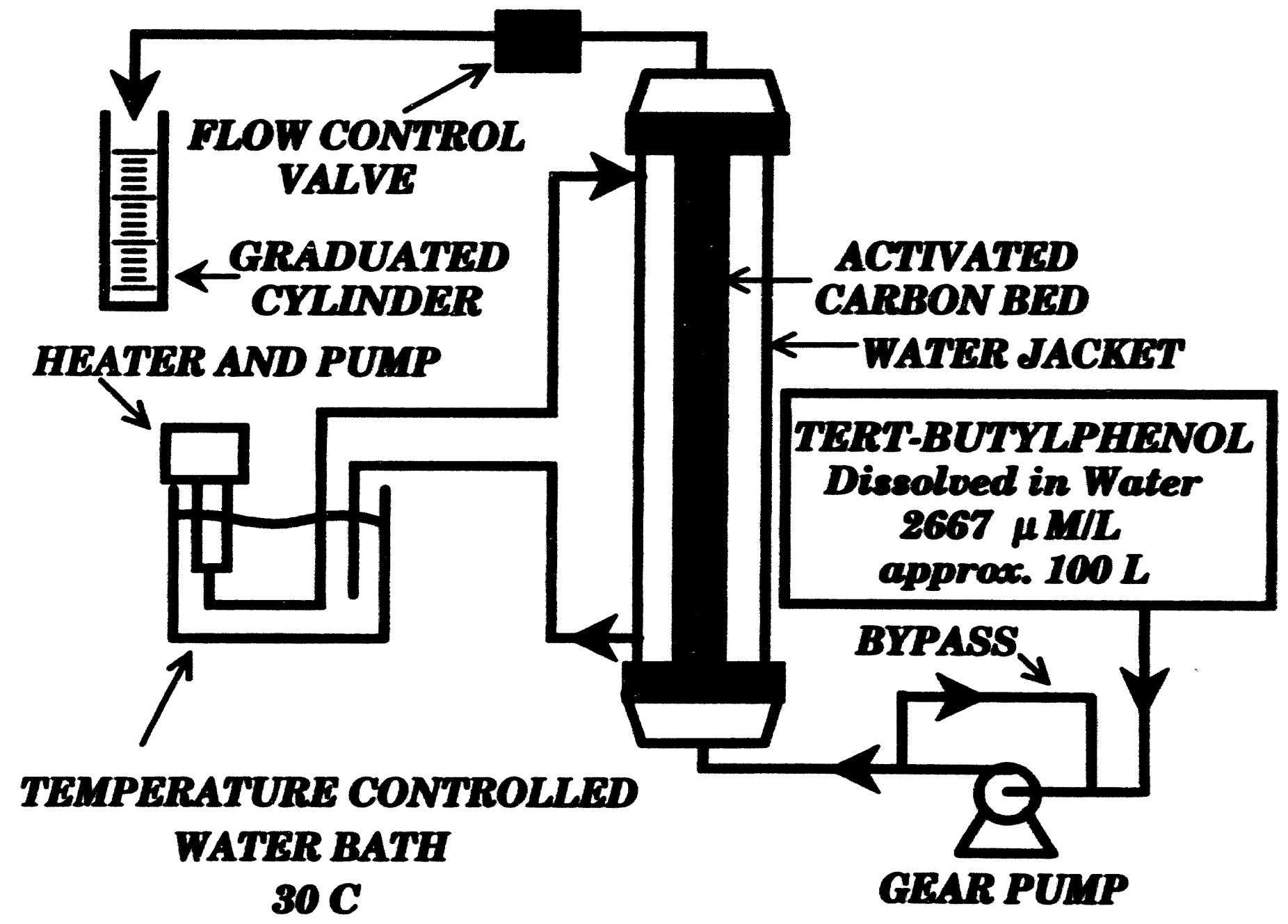

FIGURE III.9 Apparatus for the Adsorption of Tert-Butylphenol on the Carbon Bed 


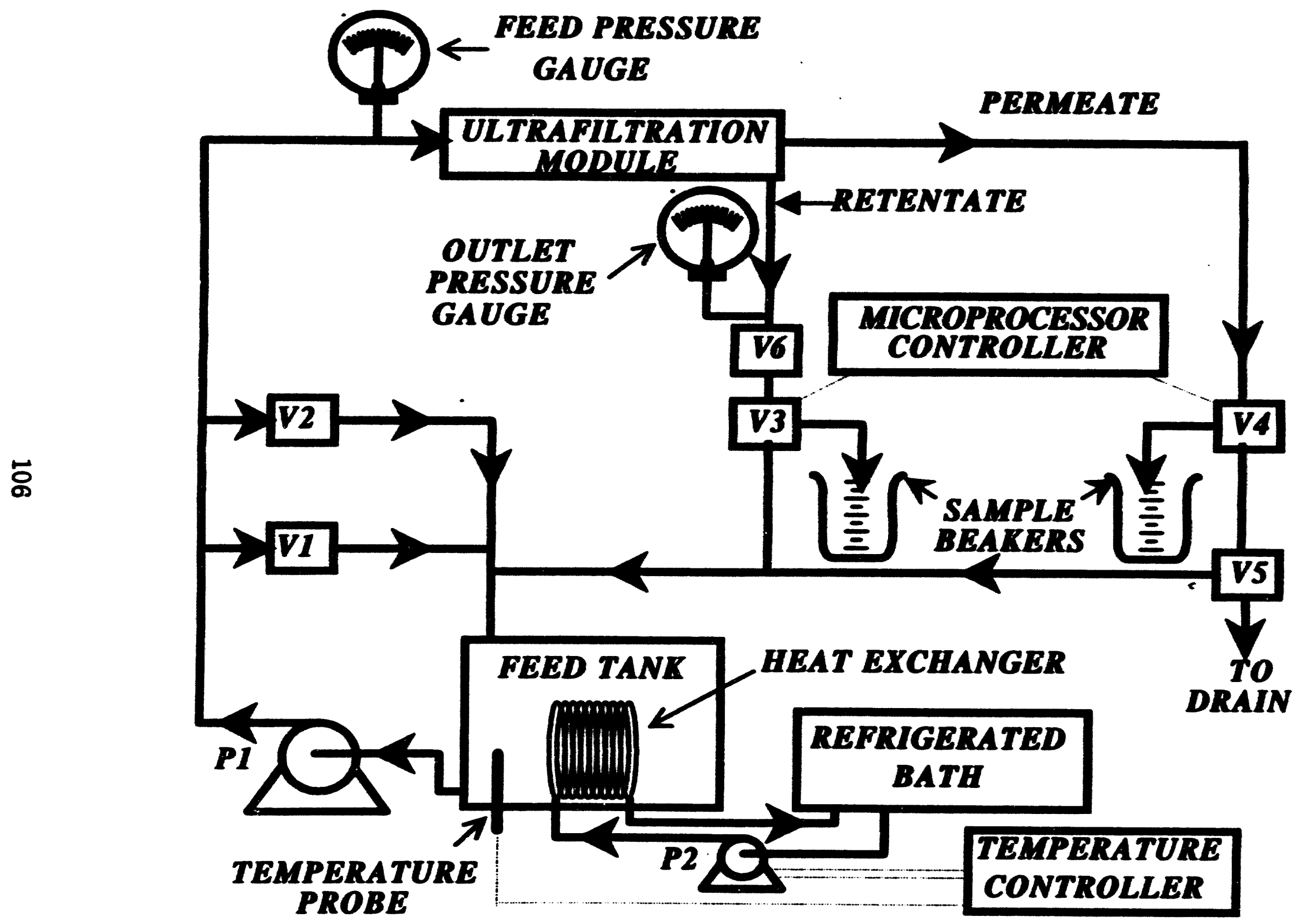

FIGURE III.10: Pilot Plant for the Micellar-Enhanced Ultrafiltration Process. 


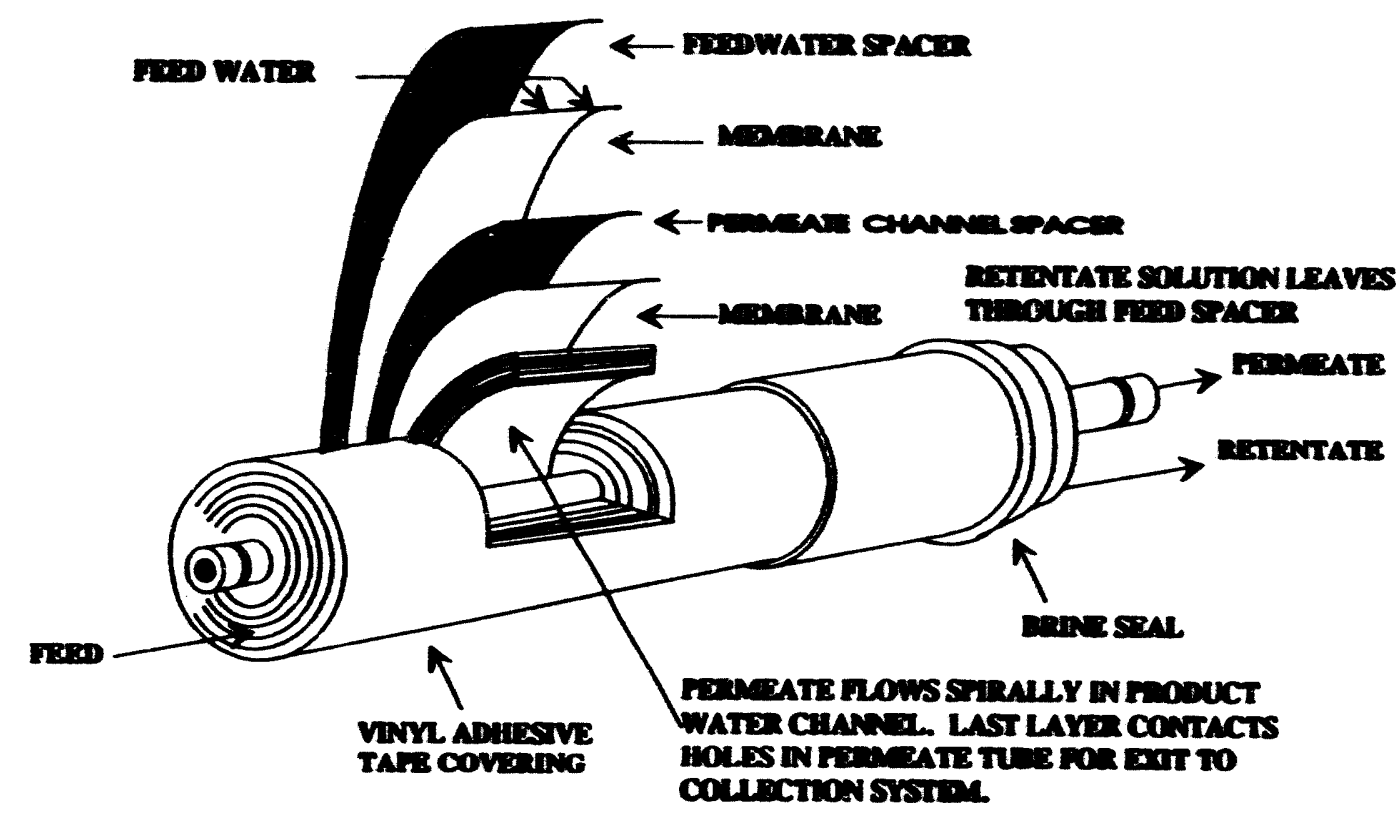

FIGURE III.11 An Illustration of the Major Componets of an Ultrafiltration Spiral Wound Membrane. 


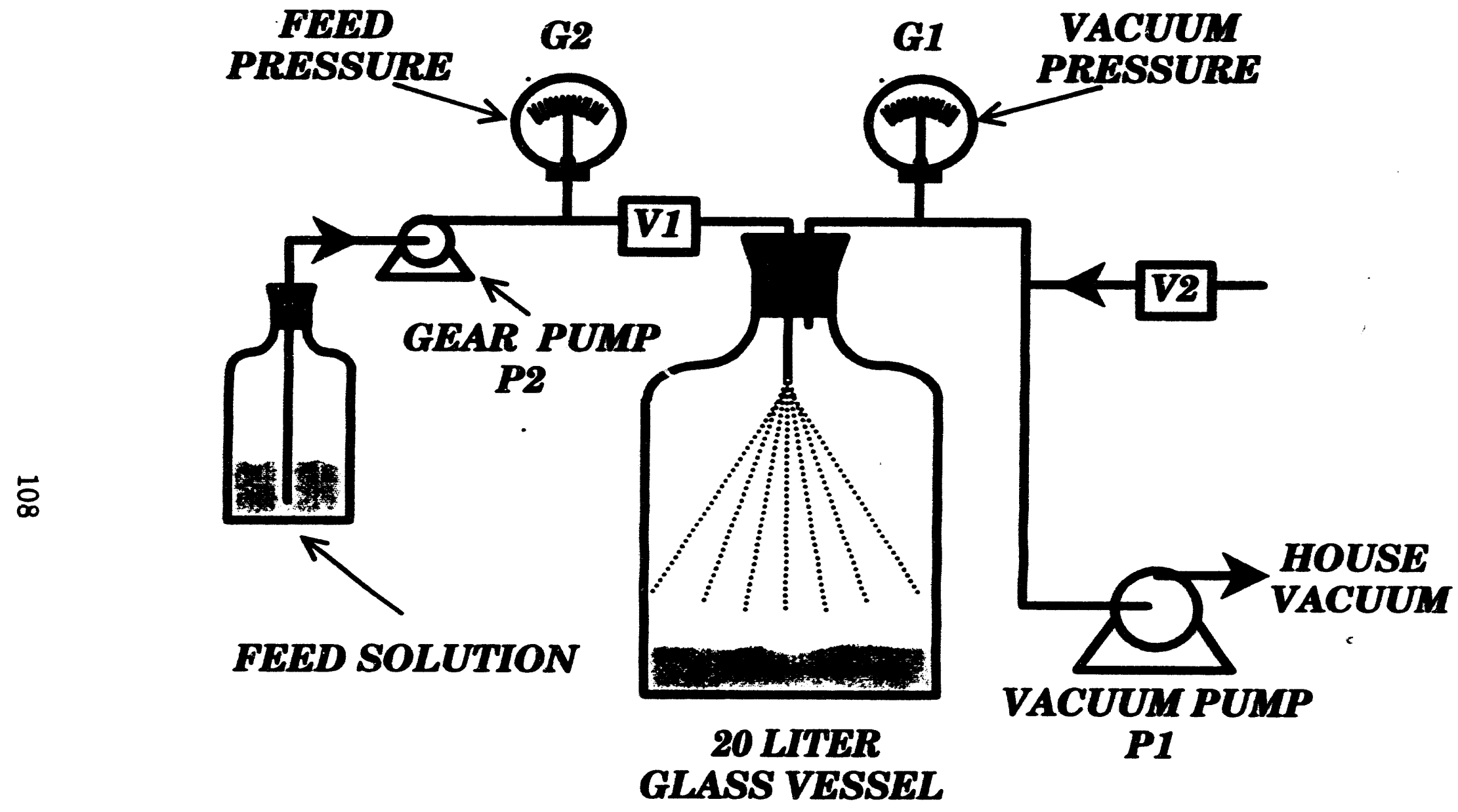

FIGURE III.12 Vacuum Stripping Apparatus 


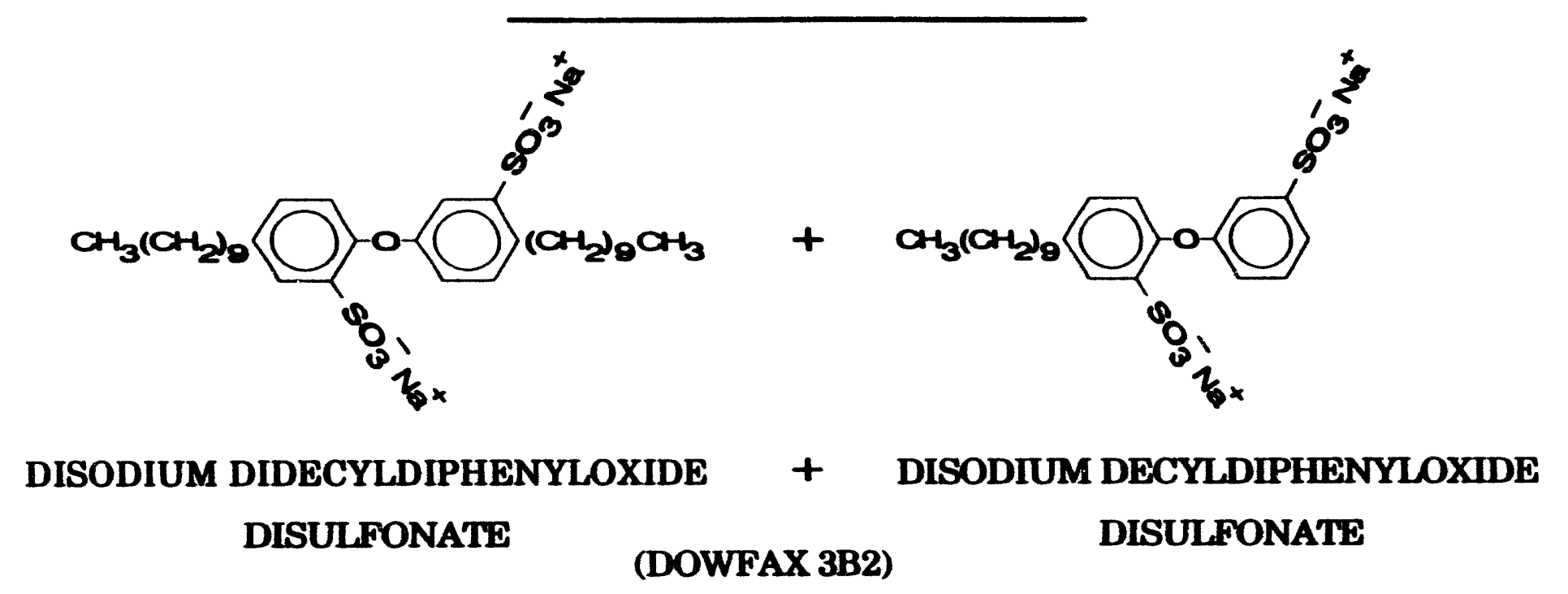

용

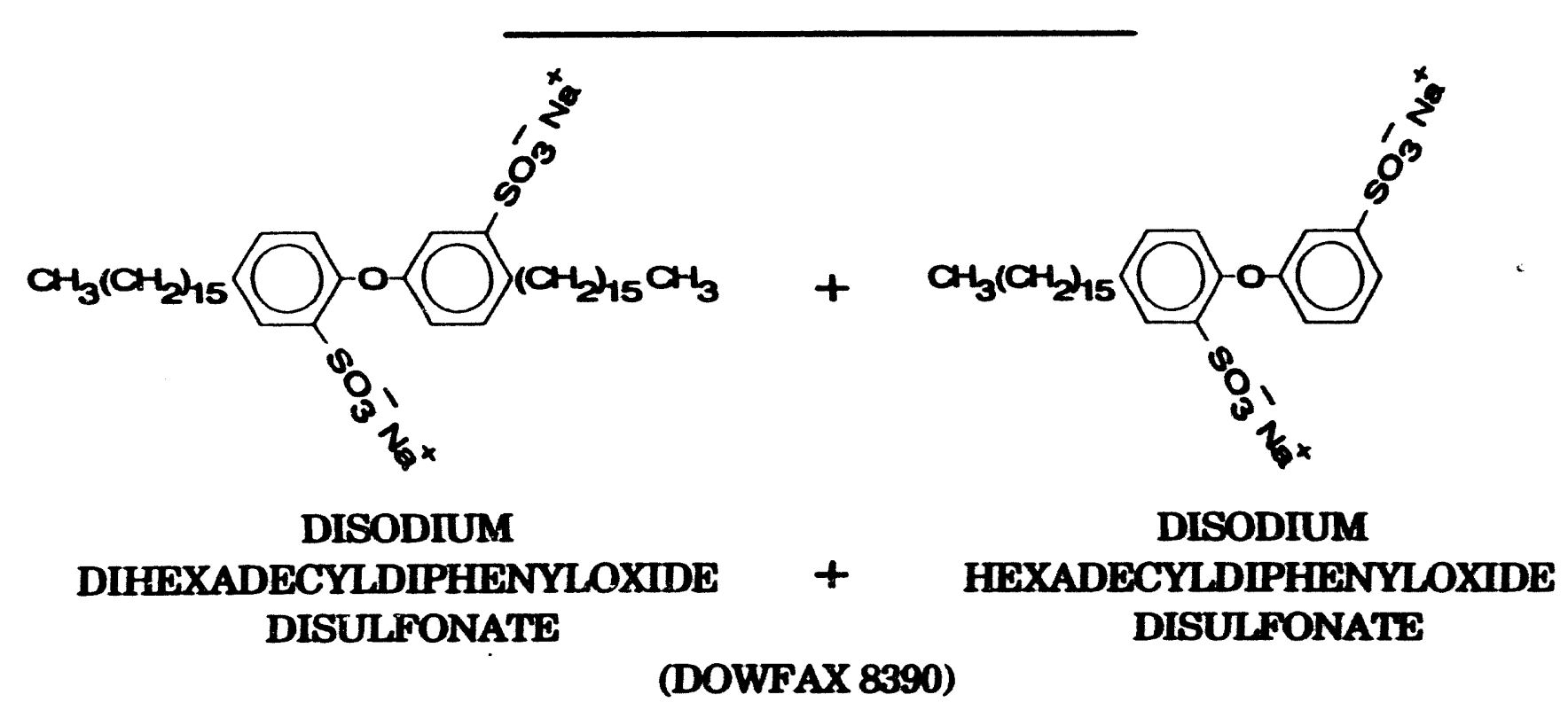

FIGURE III.13 Structures of DOWFAX Surfactants 


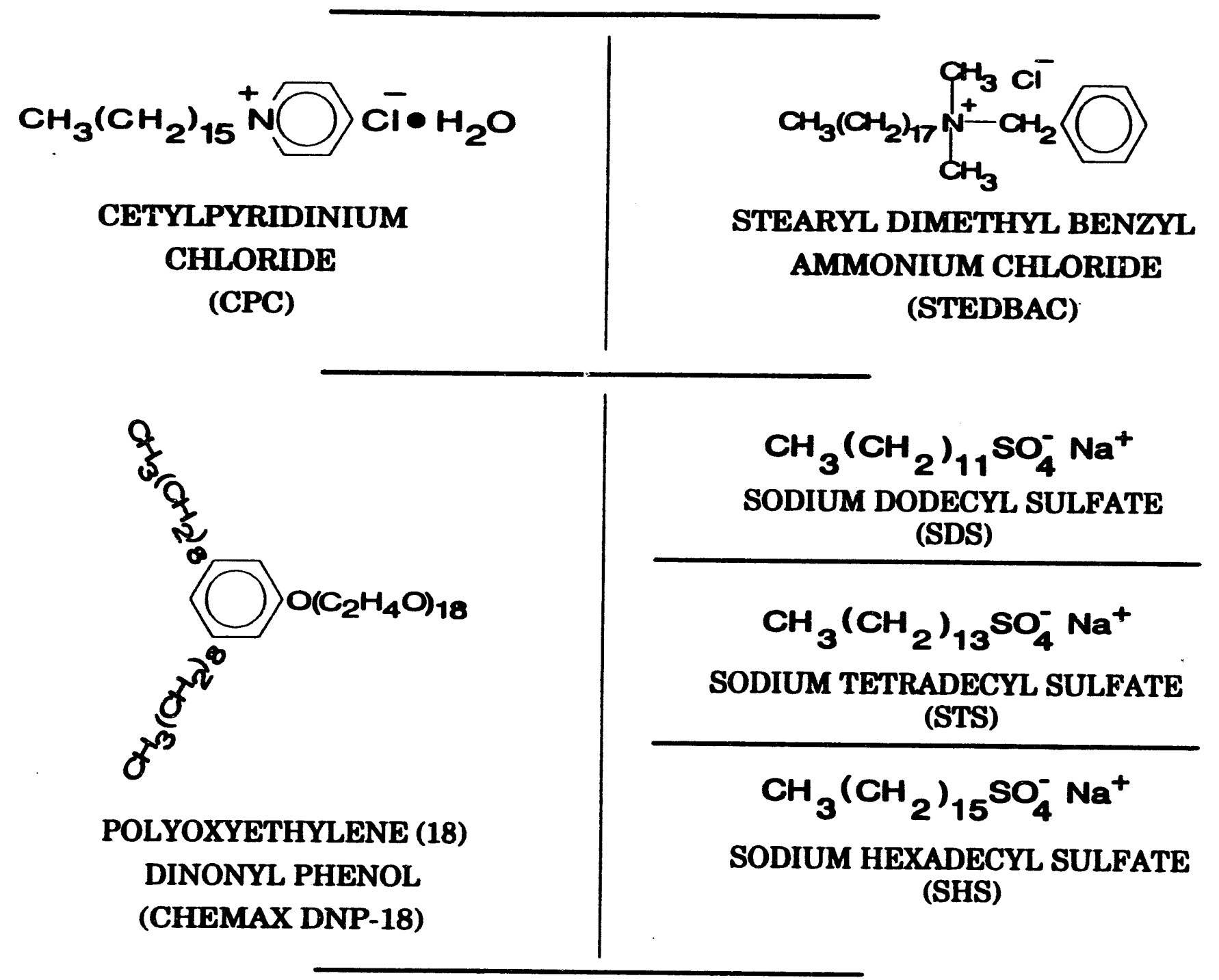

FIGURE III.14 Structures of Surfactants 
$\left(\mathrm{CH}_{3}\right)_{3} \bigcirc \mathrm{OH}$

4-tert-BUTYLPHENOL (TBP)

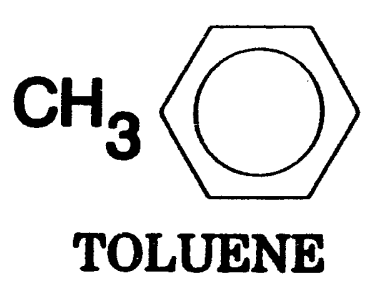

\section{$\mathrm{CH}_{3} \mathrm{CH}_{2} \mathrm{CH}_{2} \mathrm{CH}_{2} \mathrm{CH}_{2} \mathrm{O} \mathrm{CH}_{3}$ AMYL ACETATE}

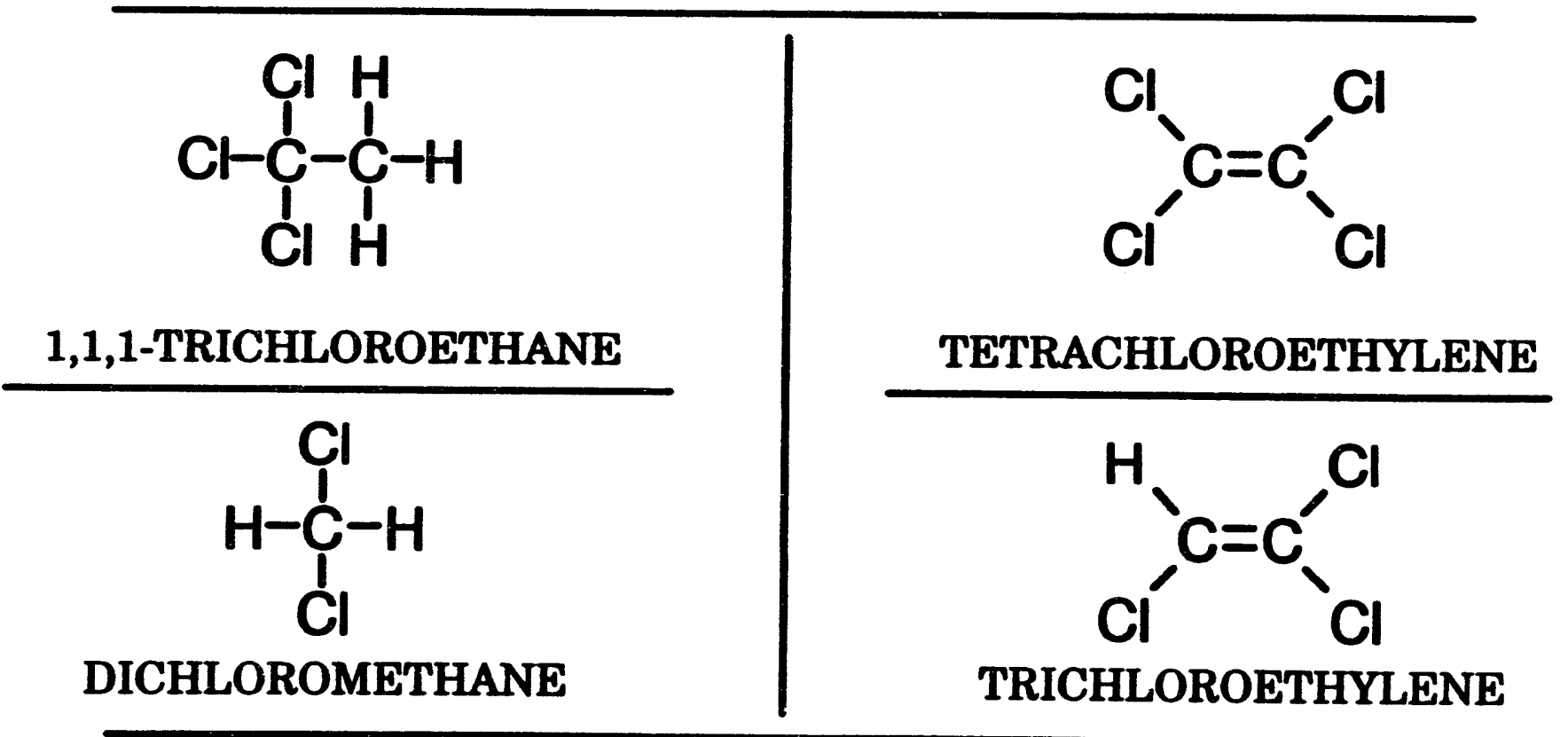

FIGURE III.15 Target Organic Solutes. 


\section{CHAPTER 4}

\section{THE USE OF SURFACTANT-ENHANCED CARBON REGENERATION TO RECOVER VOLATILE ORGANICS FROM SPENT ACTIVATED CARBON}

\subsection{Introduction}

Surfactant enhanced carbon regeneration (SECR) was briefly described in section 1.1.2. Recall that SECR depends on solubilizing the target organic adsorbed on the surface of the activated carbon as shown in Figure I.3. Solubilization is dependent on the presence of micelles, therefore, the concentration of the surfactant in the regenerant solution flowing through the carbon bed is much greater than the CMC. SECR requires three to four steps : (1) loading the target organic on the carbon bed, (2) a surfactant flood to remove the target organic form the carbon bed, (3) a water flood to remove residual surfactant form the carbon bed, and (4) drying of the bed if it is to be used for vapor phase applications.

This study was an investigation of the removal of two compounds, amyl acetate and toluene, from activated carbon and the viability of the carbon after regeneration by surfactant solutions. Both compounds are organic compounds commonly used by the painting and printing industries. Amyl acetate has a boiling point of $142^{\circ} \mathrm{C}$ and is commonly used as a leveling agent in paints and inks. Because of the high boiling point, it is difficult to volatilize this compound when adsorbed on an activated carbon bed. In hearth or thermal regeneration, in order to completely regenerate activated carbon with amyl acetate adsorbed on the 
surface, the carbon requires regeneration at temperatures approaching $900^{\circ} \mathrm{C}$ in the presence of enough oxygen to oxidize the compound without oxidizing a significant amount of the carbon. The process is energy intensive. The other compound, toluene, has a boiling point of $110^{\circ} \mathrm{C}$ and, when adsorbed on activated carbon, is readily volatilized by flowing hot gas through the carbon bed. The process is generally referred to as hot gas or steam regeneration. Both of these compounds are typical of the types of compounds that are collected by activated carbon beds for pollution control as well as solvent recovery applications. Under normal circumstances, compounds with either both high and low boiling points are adsorbed on an activated carbon bed simultaneously, therefore, the process used to regenerate an activated carbon bed may be driven by the heavy components, which are the most difficult compounds to remove.

The potential exists to regenerate activated carbon beds using a concentrated surt. tant solution to remove both high and low boiling point compounds at room temperature, which can significantly reduce the energy requirements and reduce the capital costs for the activated carbon bed vessel and associated piping since the materials are not subjected to the high temperatures of hot gas regeneration.

\subsection{Results}

The perc $\lrcorner$ nt recovery is shown at three solute loadings for toluene, in Figure IV.1, and for amyl acetate, in Figure IV.2. The recovery of toluene at three different flow rates is shown in Figure IV.3; that for amyl acetate 
is shown in Figure IV.4. The recovery of toluene at three different sodium dodecyl sulfate (SDS) concentrations is shown in Figure IV.5; that for amyl acetate at two different SDS concentration in Figure IV.6. The percentage recovery of surfactant during the water flush" step is shown at two flow rates in Figure IV.7.

\subsection{Discussion}

\subsubsection{The Effect of Solute Loading}

In considering the results in Figure IV.1 and IV.2 one naturally questions why the percent recovery under some conditions is greater than 100 percent. When the solute loading was varied, the most concentrated loading was used first, followed by subsequent lower loadings on the spent carbon. Since the purpose of these experiments was to investigate the effect of solute loading, the regeneration was not performed long enough to remove all of the solute. For example, the base case run for the toluene was at a solute loading of $0.1 \mathrm{~mL} / \mathrm{g}$ carbon. If this run is repeated a number of times, each regeneration approaches $100 \%$ recovery, even though a small level of unrecovered solute remains on the bed. However, this level of unrecovered solute is constant from run to run. When the loading is substantially reduced, this unrecovered solute at the end of a normal regeneration (approximately 47 pore volumes) is less than the unrecovered solute at the end of the higher loading run. Hence, more toluene is recovered during regeneration than was loaded on the carbon. When low 
loadings is used, only a small residual solute level from a previous run can lead to substantially greater than $100 \%$ apparent recoveries. At the point at which a regeneration is terminated, though, the residual solute is generally small, estimated to be $10 \%$ of that loaded. The important conclusion from Figures IV.1 and IV.2 is that SECR is capable of effectively regenerating carbon containing a wide range of loadings. The greater than $100 \%$ recovery is an artifact of the experimental procedure and the sequence of the experiments.

\subsubsection{The Effect of Multiple Cycle}

The columns were operated for a total of nine cycles. In order to test for degradation of the carbon due to SECR after this many cycles, some qualitative results were obtained. Specifically, no solute was observed in the condenser when the organic was being loaded on the carbon in any of these experiments. If substantial reduction in adsorption capacity were occurring, not all of the organic could be adsorbed by the carbon. Therefore, it is concluded that this novel regeneration method did not have serious deleterious effects on the performance of the carbon in cleaning up volatile organics from vapors over 9 cycles of operation.

\subsubsection{The Effect of Solute Type}

Comparison of Figures IV.1 and IV.2, IV.3 and IV.4, and IV.5 and IV.6 shows that amyl acetate is more easily recovered from the carbon than 
toluene (a given \% recovery is attained in fewer pore volumes). Recall that the first step in the regeneration process is the water flood, to displace air in the pore, to prevent foaming. Therefore, the first several data points for the first pore volume reflect the organic solute concentration in pure water in equilibrium with the organic solute on the carbon surface. This toluene concentration varied between 3.0 and $6.0 \mathrm{mM}$ during this period, while the amyl acetate concentration varies between 15 and $20 \mathrm{mM}$. This greater tendency of the amyl acetate to distribute itself in the water phase caused it to be more easily removed from the carbon by the regenerant solution. It is also well known that solutes with aromatic rings solubilize at higher levels in micelles composed of cationic surfactants than in micelles composed of anionic surfactants (e.g., SDS), due to $\pi$ electron attractions for the cationic head group (57.58). This could explain the preferential solubilization of the amyl acetate over the aromatic toluene in the anionic surfactant.

\subsubsection{The Effect of Regenerant Solution Flow Rate}

From Figures IV.3 and IV.4, the greater the regenerant solution flow rate, the more pore volumes are required to regenerate the bed to a specified level. However, the effect is small for the toluene and much larger for the amyl acetate. If the regeneration step were equilibrium limited, flow rate would have no effect (when compared on a pore volume basis). Therefore, it may be concluded that mass transfer effects are significant for both solutes, but that the toluene is mainly limited by equilibrium solubilization considerations, while mass transfer effects are important for 
the amyl acetate. Of course, a higher flow rate could result in a shorter regeneration time, even if the recovery is poorer on a pore volume basis, hence requiring more total regenerant solution. If the regenerant were being recycled after removal of the volatile, this would not be a serious obstacle to using a higher flow rate.

\subsubsection{The Effect of Surfactant Concentration in Regenerant Solution}

From Figure IV.5, the number of pore volumes required to achieve a specified percent recovery of toluene is approximately halved when the surfactant concentration in the regenerant solution is doubled from $0.1 \mathrm{M}$ to $0.2 \mathrm{M}$. This is expected if the process is equilibrium limited and the solubilization equilibrium constant is independent of solute concentration. However, the surfactant concentration has little effect on the removal of amyl acetate, as seen in Figure IV.6. These results are consistent with those from the flow rate data: the toluene removal is mainly equilibrium limited while that of amyl acetate demonstrates significant mass transfer resistances.

\subsubsection{The Causes of Mass Transfer Limitation}

Since amyl acetate has a higher molecular weight and is geometrically larger than toluene, it is expected to have a lower diffusivity. It could also have a higher activation energy for desorption from the 
carbon. Therefore, the results observed are reasonable, but more solutes need to be studied before drawing broad mechanistic conclusions.

\subsubsection{The Water Flush Step}

As seen in Figure IV.7, flow rate had little effect on the number of pore volumes required to flush the surfactant from the carbon following the regeneration step. Therefore, under these conditions, the water flush step is equilibrium limited. In this case, if cycle time needs to be minimized, a large flow rate should be used; if not, a small flow rate will minimize downstream unit size to treat this water flush stream.

\subsection{Conclusions}

The overall conclusion of this study is that SECR can recover either toluene or amyl acetate from activated carbon over a range of loadings, over a range of regenerant solution surfactant concentrations, and with a reasonable number of pore volumes of regenerant solution without the apparent loss of substantial adsorption capacity over multiple regenerations. The basic feasibility of the process has been demonstrated. 


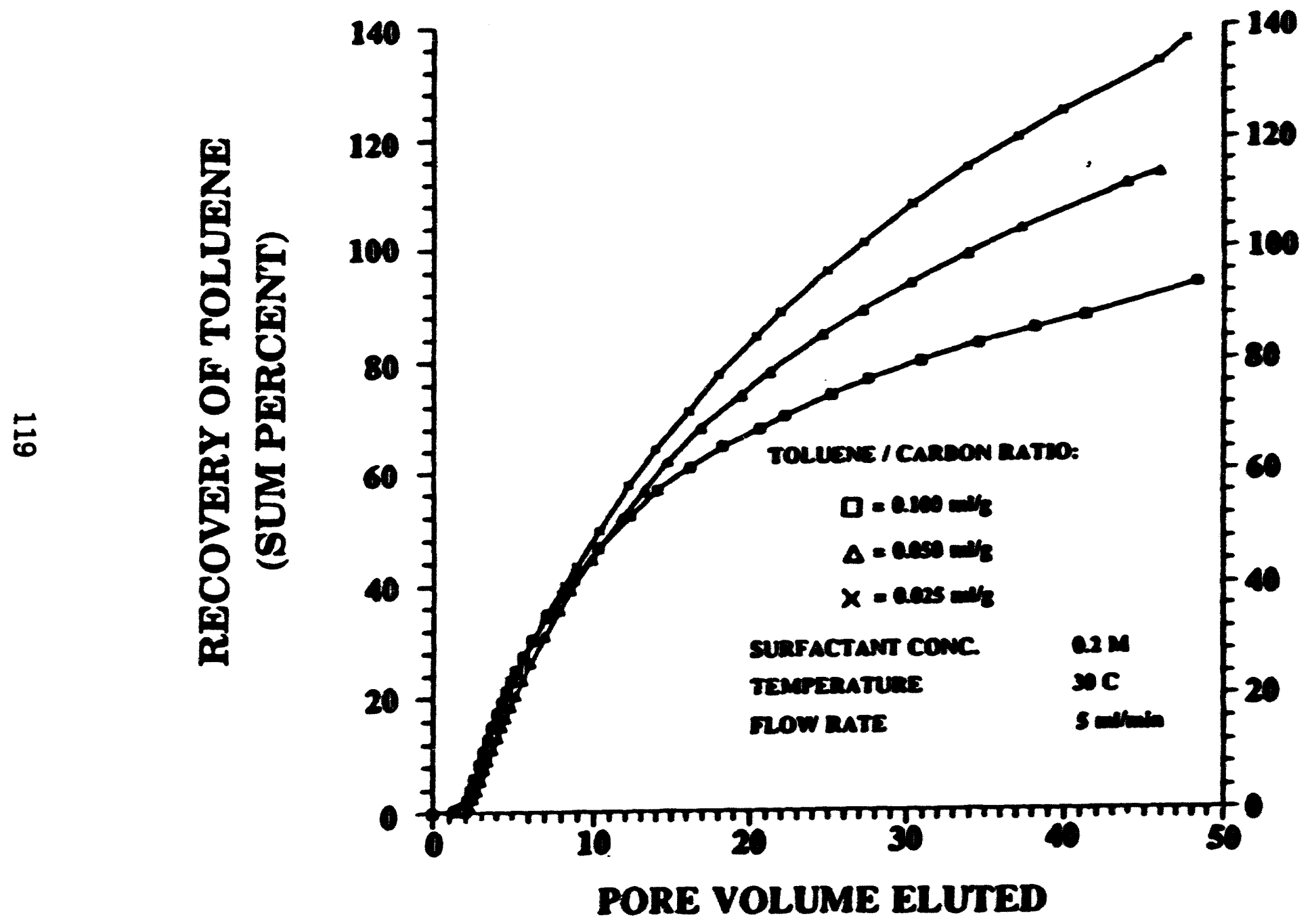

Figure IV.1: Bfiect of Toluene Loading on the Recovery of Toluene. 


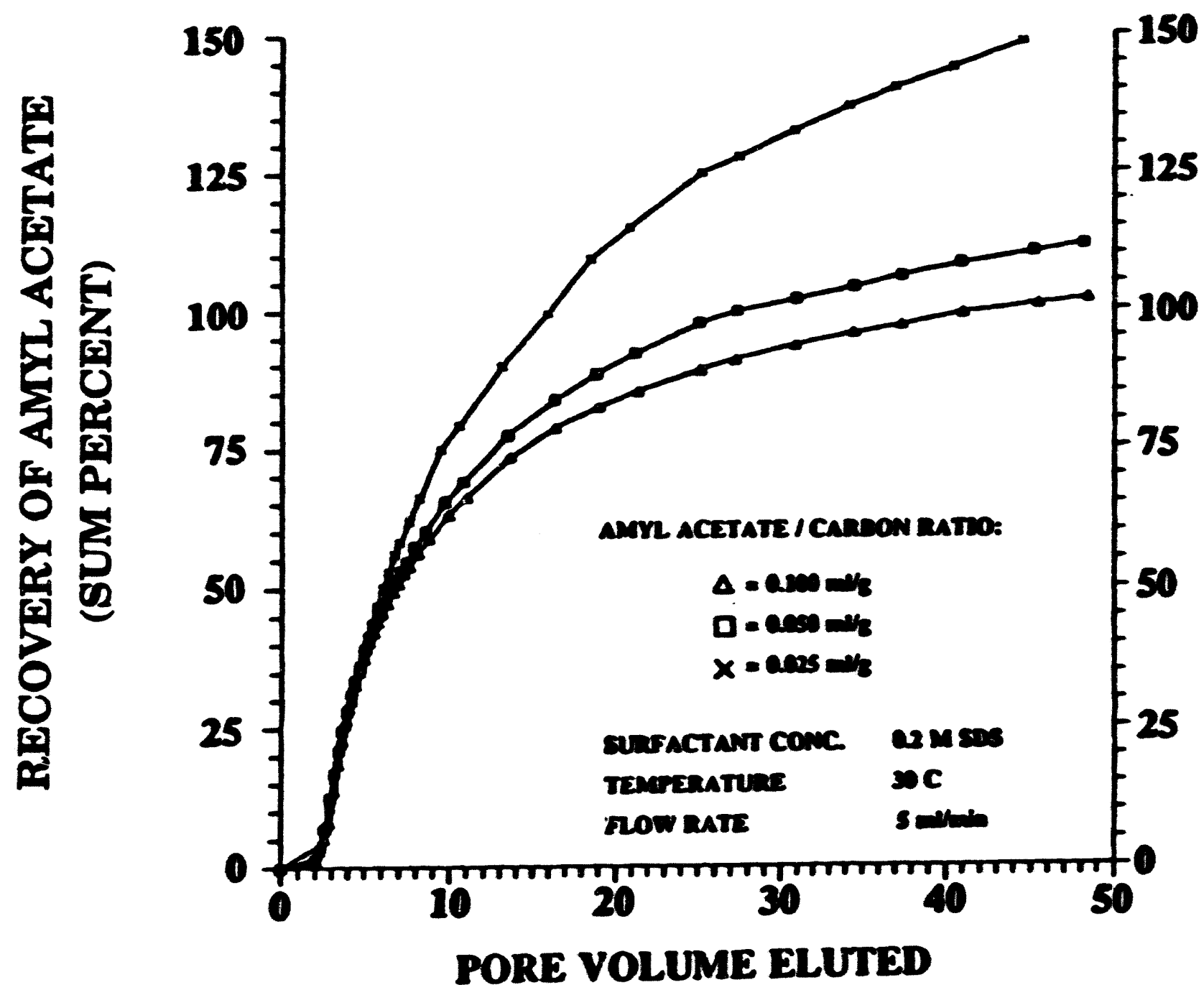

Figure IV.2: Eficet of Amyl Acetate Loading on the Recovery of Amyl Acetate. 


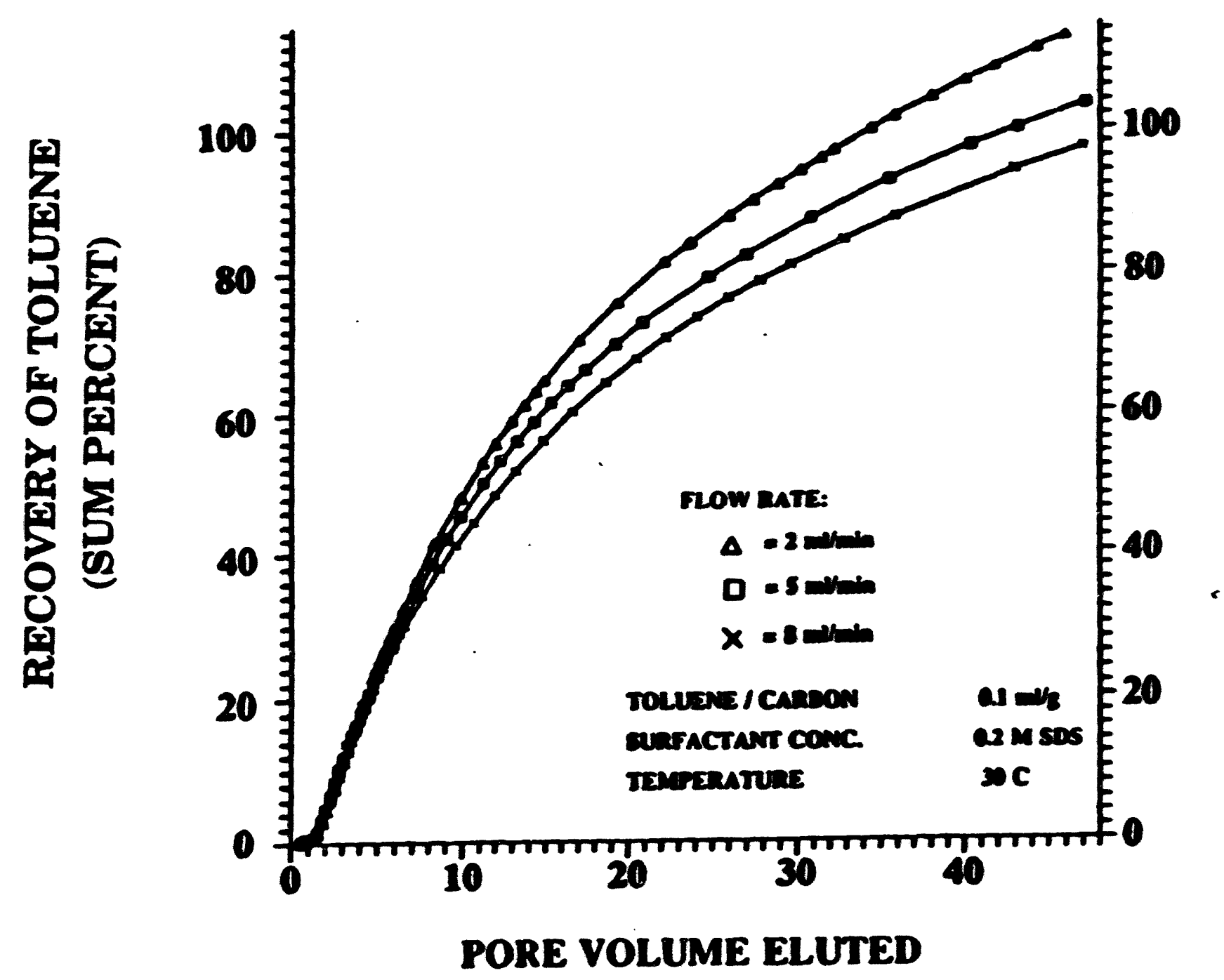

Figure IV.3: Effect of Surfactant Solution Flow Rate on the Recovery of Toluene. 


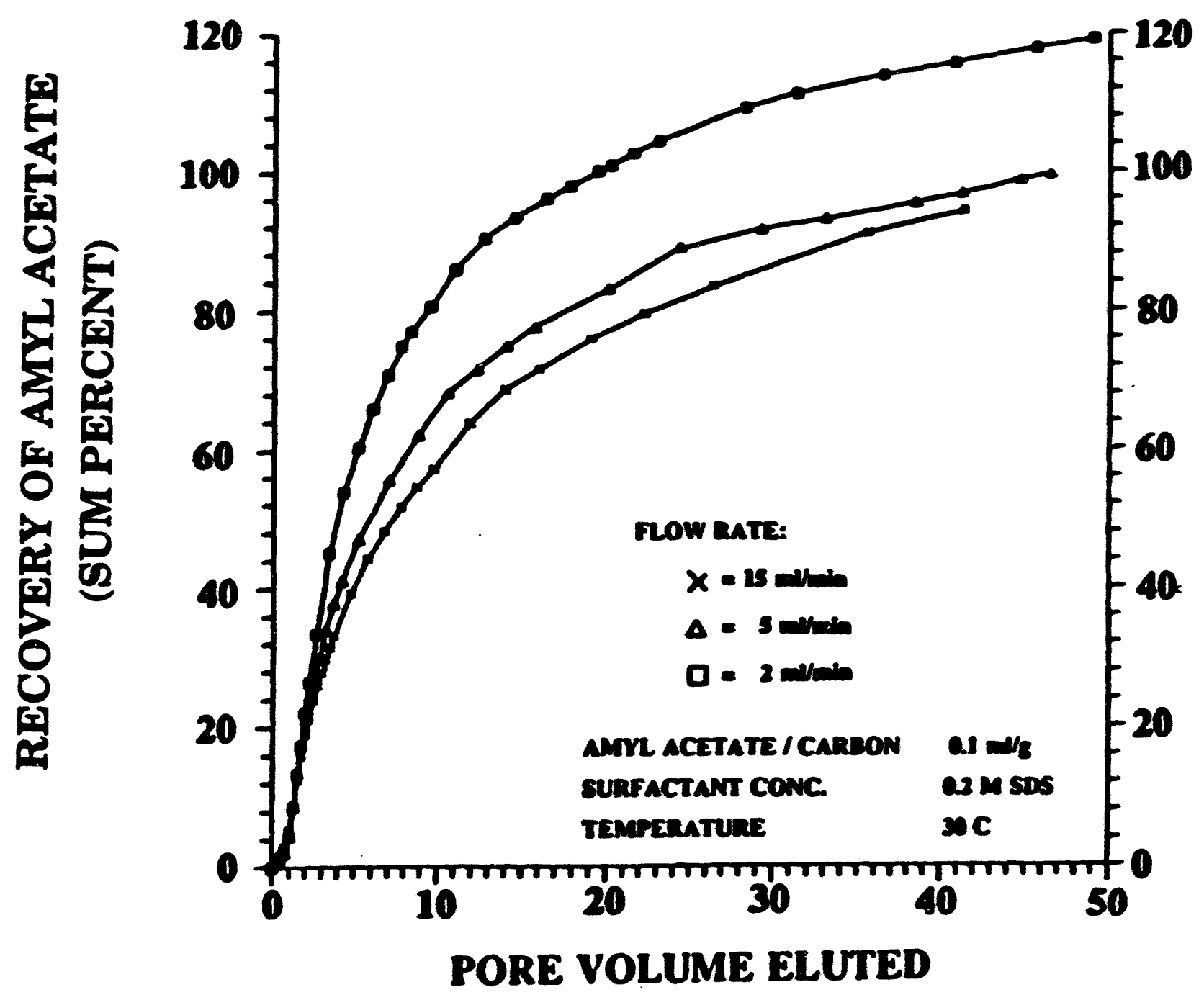

Figure IV.4: Effect of Surfactant Solution Flow Rate on the Recovery of Amyl Acetate. 


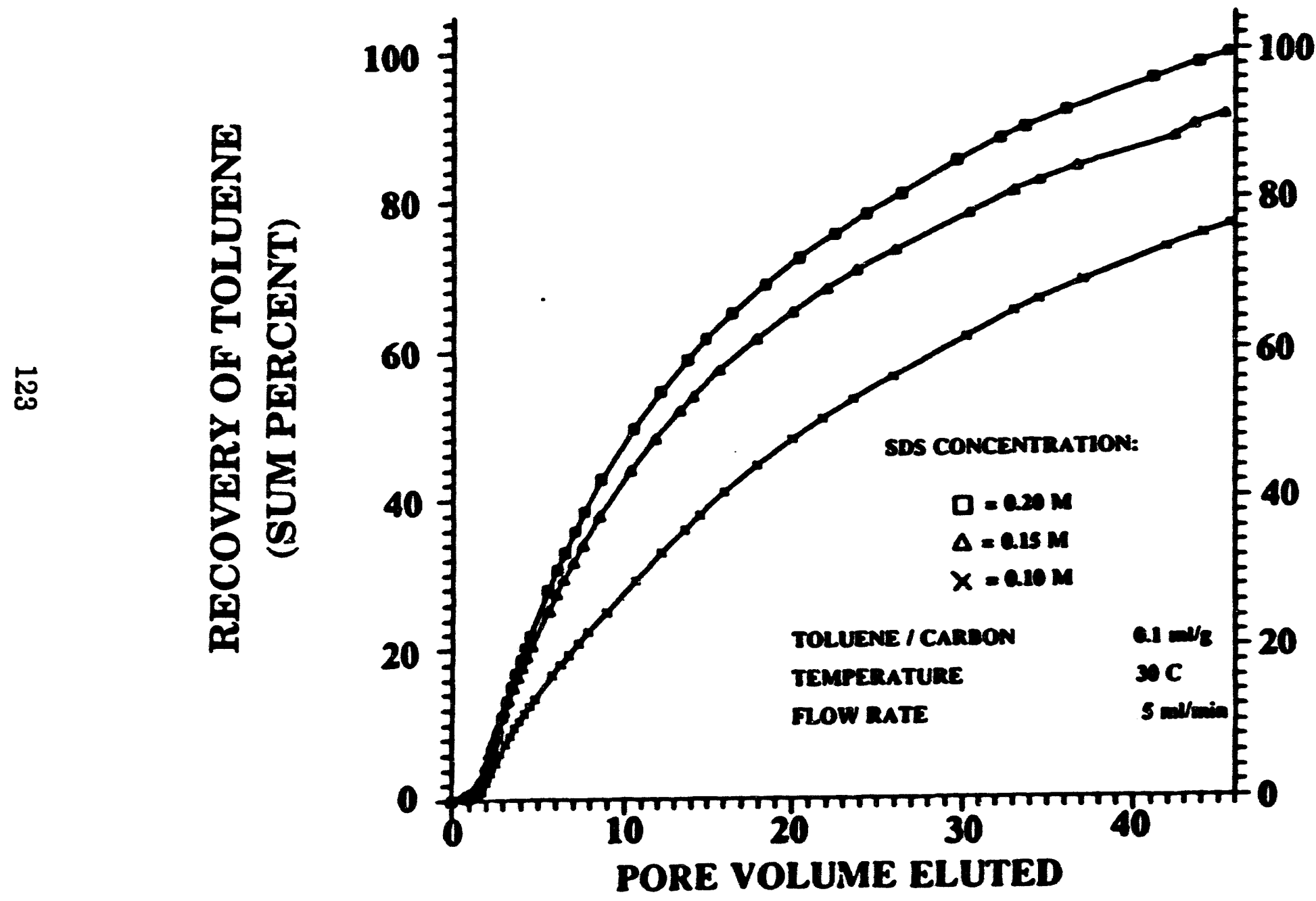

Figure IV.5: Effect of Surfactant Concentration on the Recovery of Toluene. 


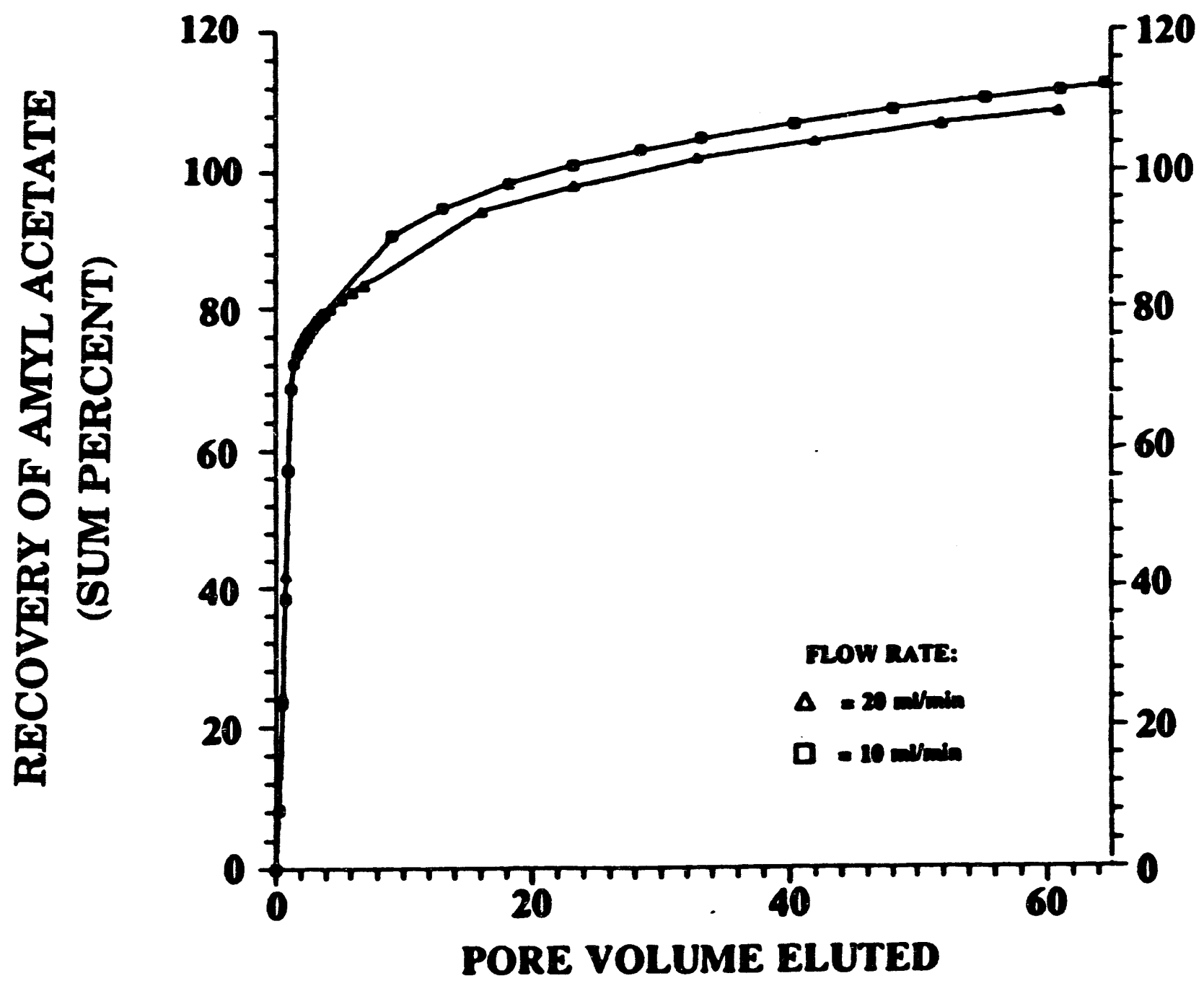

Figure IV.6: Effect of Surfactant Concentration on the Recovery of Amyl Acetate. 


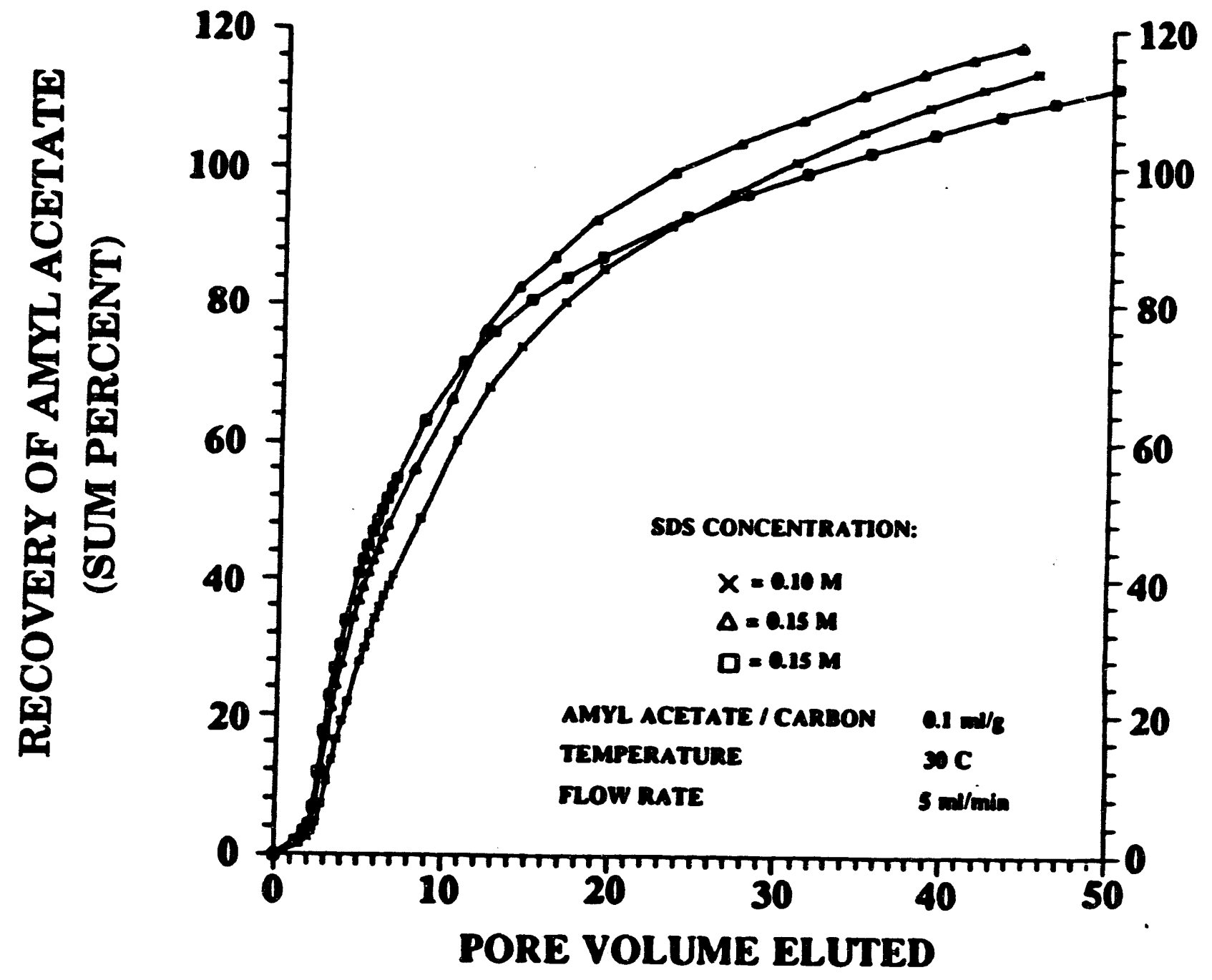

Figure IV.7: Effect of Water Flow Rate on the Recovery of Residual Surfactant. 


\section{CHAPTER 5}

\section{USE OF SURFACTANT-ENHANCED CARBON REGENERATION TO REMOVE WATER SOLUBLE ORGANICS FROM SPENT ACTIVATED CARBON}

\subsection{Introduction}

SECR, a novel separation technique, can remove organic compounds from activated carbon beds leaving the beds regenerated for reuse (59). Toluene and amyl acetate have been adsorbed from the vapor phase onto dry carbon beds and removed from the beds with an aqueous solution containing sodium dodecyl sulfate without any obvious degeneration of the carbon beds adsorptive capacity (59). TBP was adsorbed from an aqueous solution onto an activated carbon bed and was recovered from the bed using an aqueous solution containing CPC (27). However, attempts to readsorb TBP from an aqueous solution onto activated carbon beds regenerated with SDS or CPC indicated the adsorptive characteristics of the carbon bed were substantially modified and indicated there may be a significant loss of bed capacity. Preliminary indications suggested the complete removal of the surfactant from the carbon bed by the water flush step, was incomplete and may limit the utility of SECR for liquid phase applications.

There was one major difference between the carbon beds used for the adsorption of TBP from an aqueous solution and for the adsorption of toluene or amyl acetate from an air stream. The carbon beds used for the adsorption of TBP were wet because the TBP was adsorbed from an 
aqueous solution. The beds used for toluene and amyl acetate were dry when the organic vapors were adsorbed from an air stream. If the ionic surfactant were not removed from the surface of the carbon during the water flush step, it may affect the adsorptive characteristics of the carbon differently for a wet carbon bed than for a dry carbon bed. The surfactant may remain adsorbed on the carbon surface thus blocking the adsorption of TBP on the wet carbon bed. The surfactant may have recrystalized on beds that were dried for the adsorption of the organic vapors, toluene and amyl acetate. The recrystalized surfactant may not be adsorbed on the carbon surface or may occupy fewer adsorption sites than an equivalent amount of surfactant adsorbed on a wet carbon bed. This could explain why the TBP could not be readsorbed on carbon regenerated by SECR while toluene and amyl acetate were readsorbed on carbon treated by SECR.

\subsection{Results}

The desorption of CPC, by the water flush step, was investigated at three different temperatures to determine if the surfactant is removed from the carbon bed and if the rate of removal can be manipulated by changing the bed temperature. The change in the adsorptive capacity of the carbon bed, before and after SECR is used to regenerace the carbon bed, was determined for TBP. Static adsorption isotherms were also investigated to determine the affects of temperature on the equilibrium adsorption of both CPC and SDS on activated carbon. 
The static adsorption isotherms for the adsorption of CPC and SDS on activated carbon at three different temperatures are shown in Figure V.1 and Figure V.2 respectfully. The column breakthrough curves for TBP on virgin carbon and carbon which has experienced one regeneration using CPC are shown in Figure V.3. The recovery of CPC during the water flush, including not only the surfactant is adsorbed on the bed but also the surfactant solution in equilibrium with the carbon and occupying other voids in the system (pumps, tubing and valves) is shown for three different temperatures in Figure V.4. The recovery of the CPC which is adsorbed on the carbon surface, for three different water flush temperatures is shown in Figure V.5. The data in Figure V.5 is obtained from that in Figure V.4 by subtracting the surfactant in the void volume of the system.

\subsection{Discussion}

\subsubsection{The Effect of Temperature on the Equilibrium Adsorption ' of CPC and SDS on Carbon}

The equilibrium adsorption of CPC and SDS in Figure V.1 and V.2 respectfully, show maxima. An adsorption maximum and similar levels of adsorption have been observed in previous studies of CPC adsorption on activated carbon (27). The maxima observed at relatively high surfactant concentration may be due to a transition to a different micelle shape or size. The adsorption of CPC is slightly greater as the temperature is elevated from $30^{\circ} \mathrm{C}$ to $50^{\circ} \mathrm{C}$. Indicating that the adsorption is more endothermic 
than micelle formation. This may indicate that interactions between the carbon and CPC are not totally hydrophobic in nature. The adsorption of SDS does not show a significant temperature dependence but does show relatively high loading at low equilibrium concentrations. However, the SDS is a commercial grade and appears to have salt as an impurity that may have stabilized surface aggregates, increasing adsorption. The loading levels for both SDS and CPC are comparable and the shapes of the isotherms are similar. However, CPC does adsorb less as the equilibrium concentration decreases while the SDS adsorption appears to remain nearly constant.

\subsubsection{The Effect of Temperature On The Desorption of CPC}

The percentage of CPC recovered is illustrated in Figure V.4 as a function of pore volumes of water pumped through the bed. The steep rise in the percent recovery for the first few pore volumes at all three temperatures is primarily due to the recovery of surfactant from the void volume of the system. The appearance of the temperature dependence in the first few pore volumes is due to the variation of void volume and final surfactant concentration in equilibrium with the carbon for each of the three systems. The percent recovery of CPC as a function of pore volume for the surfactant adsorbed on the carbon surface is shown in Figure V.5 and illustrates that CPC desorption exhibits no obvious temperature dependence for the temperatures studied. A significant observation is that only 11 percent or 7.5 grams of the surfactant was removed from the bed at 
$30^{\circ} \mathrm{C}$ bed. The difficulty in desorbing the CPC from the carbon is consistent with chemisorption of the surfactant. Other surfactant adsorption work has shown that anionic, cationic, and nonionic surfactants have relatively strong hydrophobic interactions with activated carbon as indicated by high exothermic heats of adsorption (60). However, little temperature dependence of adsorption was observed and so, high heats of adsorption were not observed here, as would be expected if chemisorption were occurring.

The slow desorption of surfactants from the dynamic bed studies is consistent with the relatively high equilibrium adsorption at the lower concentrations . Dynamic desorption studies of SDS probably would not yield results significantly different than those of CPC considering the degree of loading and similarities between the two groups of isotherms.

\subsubsection{Dynamic Experiments}

The breakthrough curves in Figure V. 3 illustrate the bed capacities for adsorption of TBP on virgin carbon and carbon previously regenerated using CPC. The virgin carbon had adsorbed approximately 64.8 grams of TBP when the effluent concentration exceeded $30 \mu$ moles $/$ and was detected after $162 \mathrm{~L}$ of TBP solution was pumped through the bed. The carbon previously exposed to CPC adsorbed approximately 7.6 grams of TBP when breakthrough was initially detected after $19 \mathrm{~L}$ of solution was pumped through the bed. The capacity of the bed, previously regenerated by CPC, was 11.7 percent of the capacity of a virgin carbon bed. Therefore, 
the residual CPC remaining on the $30^{\circ} \mathrm{C}$ carbon bed, even after 500 pore volumes of water flush, significantly reduced the adsorption of TBP.

\subsubsection{Other Considerations}

The two surfactants studied have a higher affinity for the carbon surface than for the aqueous solution under the conditions that were evaluated. The ideal surfactant would not adsorb on the surface of the carbon or could be manipulated into desorbing if it were to adsorb. It has been shown that the adsorption density of SDS on activated carbon can be modified by changing the salt concentration (61). A potential surfactant candidate may be one that is manipulated into adsorbing on the carbon surface by using an elevated salt concentration to stabilize the surfactant aggregate on the surface and to reduce the salt or counterion concentration by flushing with water when desorption is desired.

The successful use of nonionic surfactants for carbon regeneration is doubtful. A study of competitive adsorption of p-n-octylphenol-polyglycol (a nonionic surfactant) and SDS showed that the nonionic surfactant had a higher affinity for the carbon surface by displacing the SDS from the surface (61).

A surfactant is composed of a hydrophobic moiety and a hydrophilic moiety. The nature of the interaction of surfactants with the carbon. surface being hydrophobic, it is difficult to visualize a type of surfactant that would not have significant interactions with the carbon surface. 


\subsection{Conclusions}

Temperature has little effect on surfactant adsorption levels on activated carbon. The effect of temperature on the desorption of CPC was negligible. The effect of temperature on the equilibrium adsorption of CPC were small but were not enough to significantly enhance the dynamic desorption process. However, the change in adsorption with temperature does indicate that the hydrophobic interaction between CPC and the carbon surface is not the only significant interaction occurring. The effect of temperature on the equilibrium adsorption of SDS was negligible.

The relatively high adsorption levels at low equilibrium surfactant concentrations indicates that SDS and CPC have a much higher affinity for the carbon surface than for the aqueous phase, making desorption of these surfactants by flushing with water difficult.

The results also reinforce the proposition that the adsorption is hydrophobic since the two surfactants investigated have opposite charges and similar adsorptive loading on the carbon. The breakthrough curves of TBP indicate that CPC has a a higher affinity for the carbon surface than does TBP and that residual adsorbed CPC blocks the adsorption of TBP.

Results support the hypothesis that ionic surfactant remaining on a dry activated carbon bed after SECR, may exist in a crystalline form occupying fewer adsorption sites than an equal amount of surfactant on the carbon surface in an aqueous environment. 


\section{CHAPTER S}

USE OF SURFACTANT-ENHANCED CARBON REGENERATION TO REMOVE

WATER SOLUBLE OROANICS FROM SPENT ACTIVATED CARBON $\ldots \ldots \ldots \ldots \ldots \ldots \ldots \ldots . . \ldots$

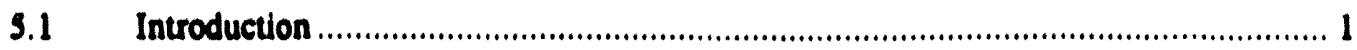

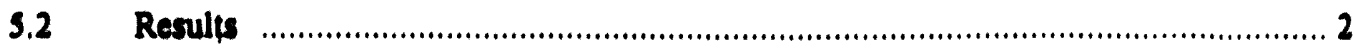

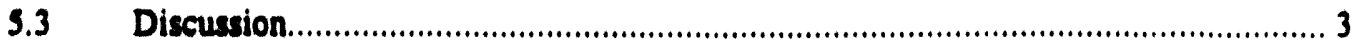

5.3.1 The Effect of Temperature on the Equilibrium Adsorption of CPC and SDS on Carbon.

5.3.2 The Effect of Tempenture On The Desorption of CPC............................. 4

5.3.3 Dynamic Experiments ........................................................................... 3

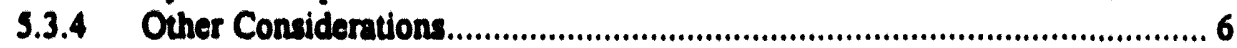

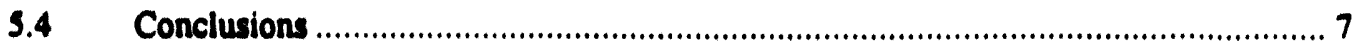

59. Roberts, B.L., Scamehorn, J.F., "Use of Surfactant-Enhanced Carbon Regeneration to Remove Volatile Organics from Spent Activated Carbon", in Surfactant-Based Separation Processes, (Scamehorn, J.F., Harwell, J.H., Ed.), Chapter 8, Buttersworth, New York, (1988).

60. Lafrance, P., Dusart, O., Mazet, M., 7 th International Symposium on Surfactants in Solution, Ottawa, Canada, 1988.

61. Schwuger, M.J., Smolka, H.G., Colloid and Polym. Sci., 1977, 25, 589. 


\section{ADSORPTION OF CETYL PYRIDINIUM CHLORIDE}

ON ACTIVATED CARBON

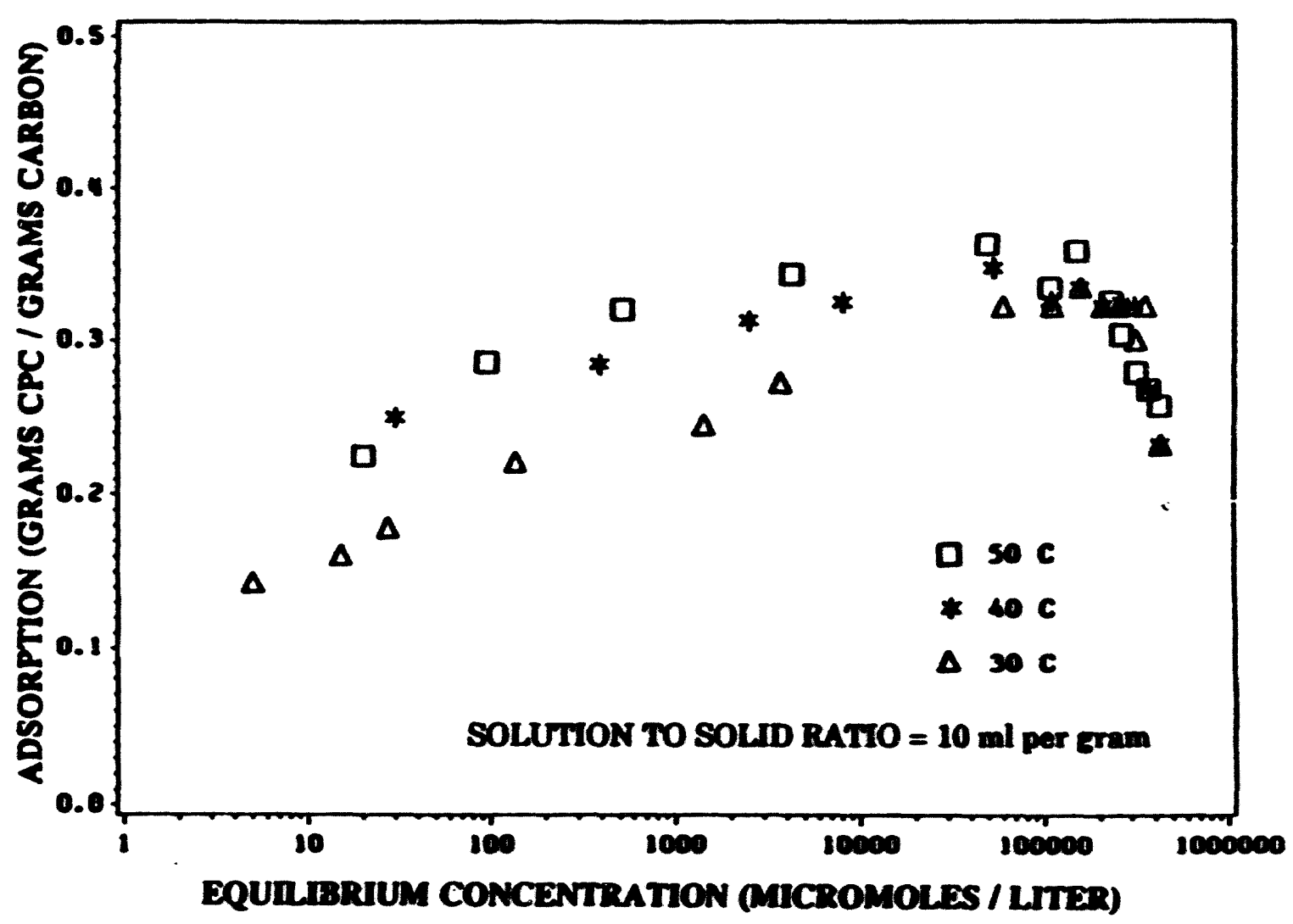

Figure V.1: Adsorption of Cetyl Pyridinium Chloride on Activated Carbon 
ADSORPTION OF SODIUM DODSCYL SULFATE

ON ACTIVATED CARBON

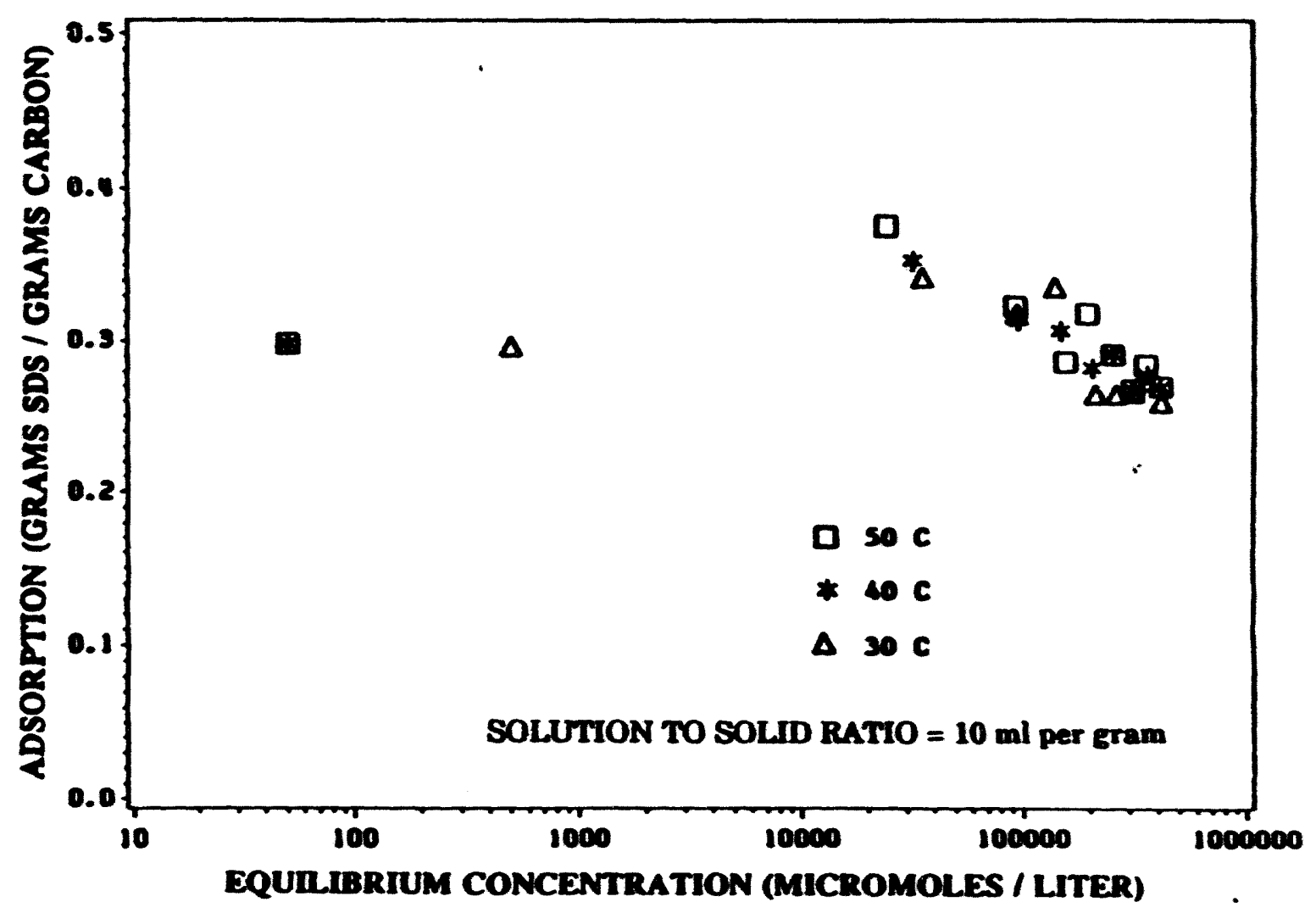

Figure V.2: Adsorption of Sodium Dodecyl Sulfate on Activated Carbon 


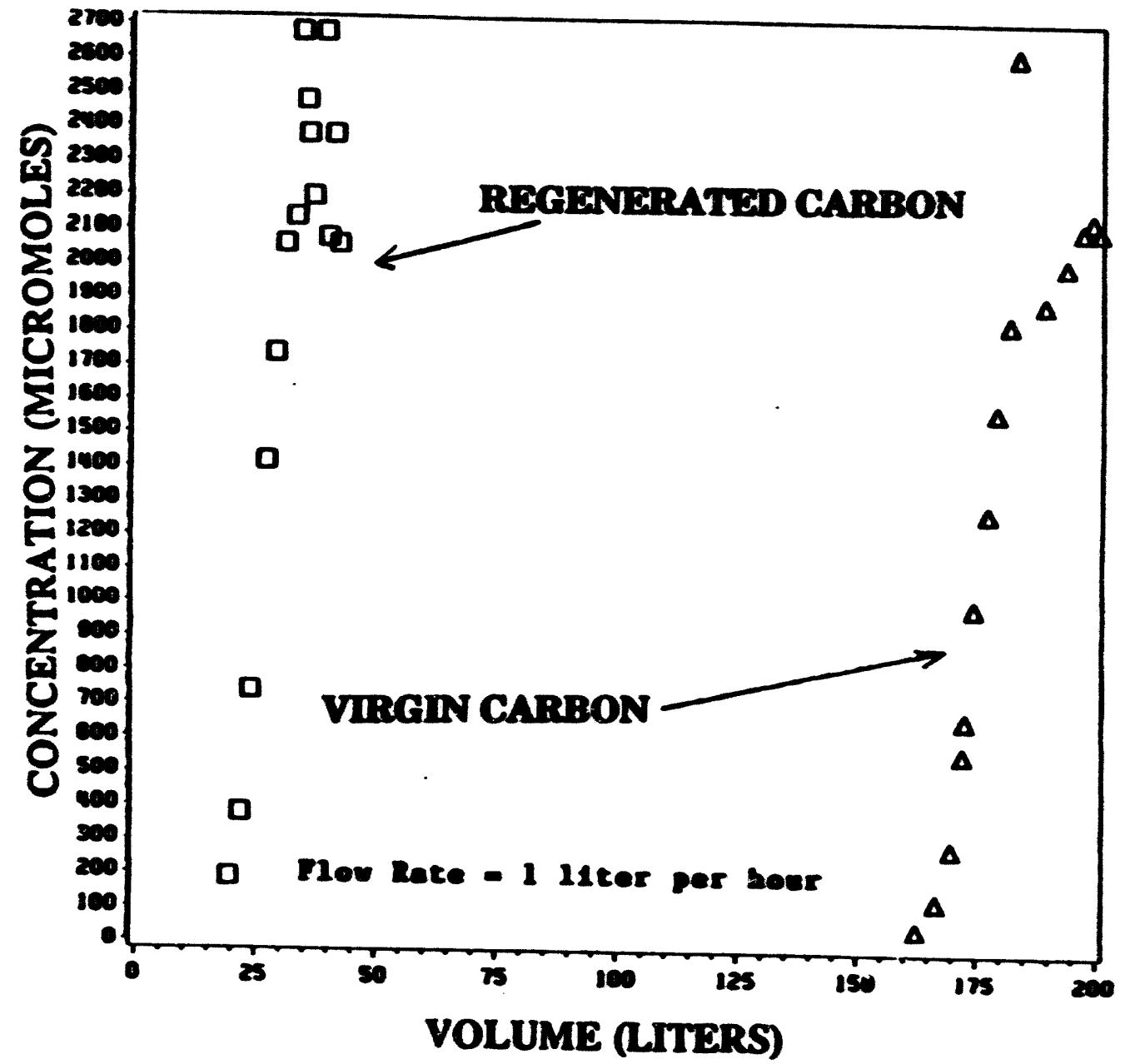

Figure V.3: Breakthrough Curves for Tert-Butylphenol on Surfactant Regenerated Carbon and Virgin Carbon 


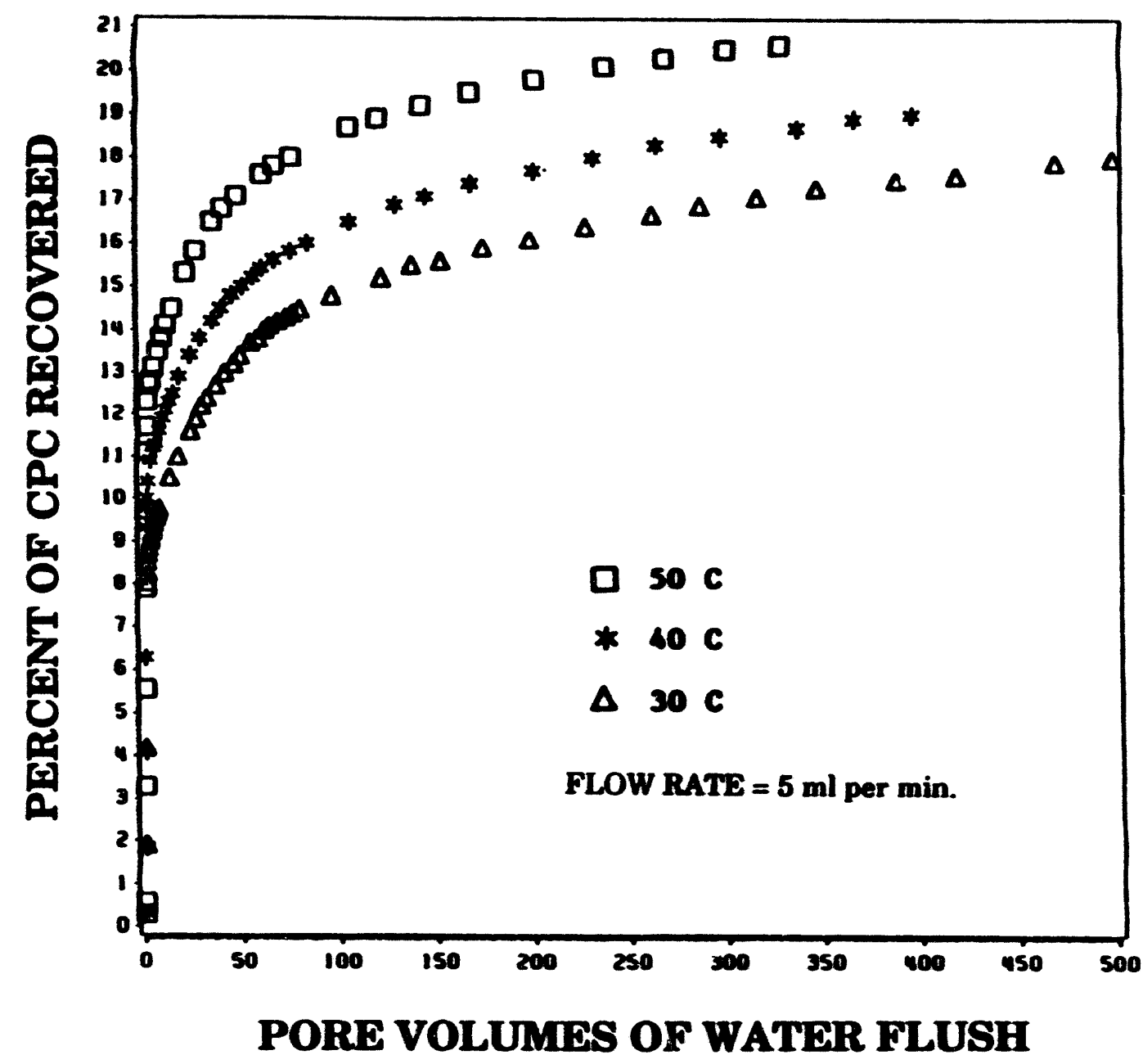

Figure V.4: Percentage Recovery of Cetyl Pyridinium Chloride from the System During Water Flush Step 


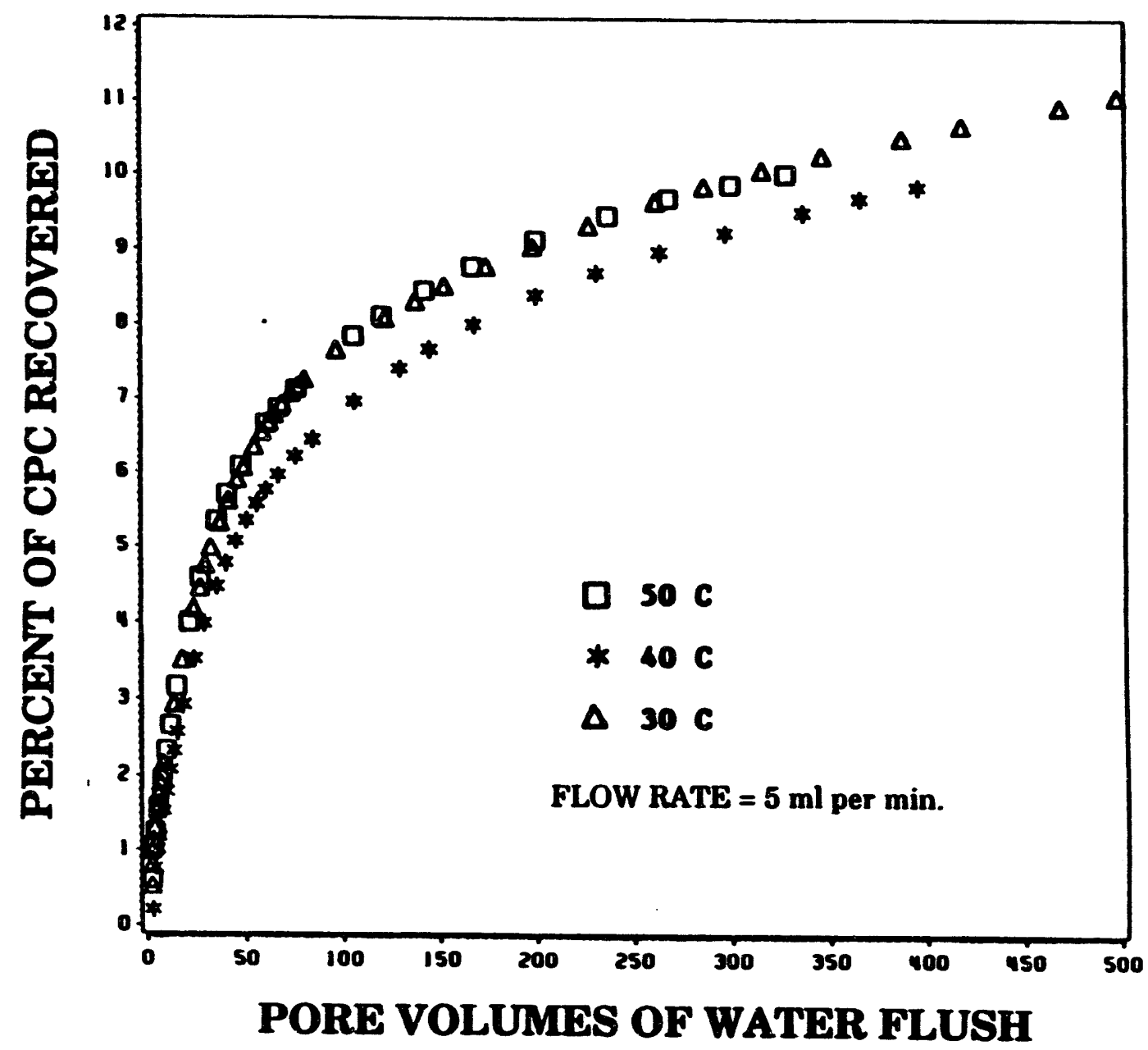

Figure V.5: Percentage Recovery of Cetyl Pyridinium Chloride from the Carbon Surface During Water Flush Step 


\section{CHAPTER 6}

\section{SCALE UP OF MICELLAR-ENHANCED ULTRAFILTRATION FOR THE RECOVERY OF TARGET MULTIVALENT IONS AND NOÑIONIC ORGANICS}

\subsection{Introduction}

Our laboratory has carried out investigations of the effectiveness of MEUF in removing a number of charged species and organics from water using a stirred cell ultrafiltration apparatus $(12,13,15,17,19,21,22)$. Table VI.1 illustrates the rejection of some typical materials using MEUF from stirred cell experiments or extrapolated from equilibrium solubilization measurements, an extrapolation which has been experimentally justified .

The rejection values in Table VI.1 range from 94.3 to 99.9 percent. MEUF can provide an excellent separation with a high percentage of the target organic solubilizing in the micelle or the charged targret ion binding to the micelle and rejected by the membrane to remain in the retentate.

The purpose of this study is to investigate MEUF, on a pilot plant scale, using commercially available off-the-shelf equipment. The spiral wound ultrafiltration unit has significantly more membrane surface area than the stirred cell which was used for bench-scale studies. Spiral wound and hollow fiber are the two most common commercial scale ultrafiltration module types (28). The pilot plant is capable of operating in a total recycle mode with all of the permeate and retentate recycling back to the feed, permitting attainment of an unambiguous steady state. 
TABLE VI.1

COMPARISON OF PERMEATE CONCENTRATIONS OF ORGANIC AND METAL DOLLUTANTS IN MEUF

\begin{tabular}{|l|c|c|}
\hline \multicolumn{1}{|c|}{ POLLUTANT } & $\begin{array}{c}\text { POLLUTANT CONCENTRATION } \\
\text { IN THE PERMEATE } \\
\text { [mM] }\end{array}$ & REJECTION \\
\hline Phenol & 1.42 & 94.3 \\
\hline m-Cresol & 0.526 & 97.9 \\
\hline 4-Tert-Butylphenol & 0.0767 & 99.7 \\
\hline Toluene & 0.80 & 96.8 \\
\hline Chlorobenzene & 0.49 & 98.0 \\
\hline n-Octanol & 0.141 & 99.4 \\
\hline Zinc (II) & 0.037 & 99.9 \\
\hline Copper (II) & 0.037 & 99.9 \\
\hline Cadmium (II) & 0.037 & 99.9 \\
\hline Chromate (-II) & 0.037 & 99.9 \\
\hline
\end{tabular}

Conditions: Retentate [Pollutant] $=25 \mathrm{mM}$; Feed [Surfactant] $=250 \mathrm{mM}$; Pressure $=414 \mathrm{kPa}$; Membrane - 1K to 20K Molecular Weight Cutoff; Temperature $=30 \mathrm{C}$

\subsection{Results}

The characteristics of a continuous MEUF process were studied using spiral wound membranes and two surfactants, CPC and SDS. A cationic surfactant, CPC, and an anionic surfactant, SDS, were used with TBP. The separation of chromate $\left(\mathrm{CrO}_{4}^{-2}\right)$ was studied using CPC while SDS was used to separate copper $\left(\mathrm{Cu}^{+2}\right)$ and cadmium $(\mathrm{Cd}+2)$ from water. Direct comparisons of permeate concentrations from spiral wound 
membrane and either stirred cell or semiequilibrium dialysis (SED) studies are discussed. Data from SED yeilds the equilibrium concentration of target organic and target metal in the permeate (19).

The flux results are shown in Figures VI. 1 through VI.3 for CPC and TBP for the spiral wound membrane. A direct comparison between the stirred cell (34) and spiral wound membrane flux results for CPC and TBP using 5K MWCO membranes are shown in Figure VI.4 through VI.6. The effect of membrane MWCO on TBP, CPC, and SDS permeate concentrations are shown in Figure VI.7 through Figure VI.10.

The permeate surfactant concentrations for SDS and CPC in the presence of a target metal for the spiral wound membrane module are shown in Figure VI.11 through Figure VI.13. The ratio of surfactant to target metal in the feed is shown in Figure VI.14 through Figure VI.16. The permeate copper concentration for the spiral wound system using 10,000 MWCO membranes is shown in Figure VI.17. The percent rejection of copper for the spiral wound 10,000 MWCO membrane is shown in Figure VI.18. A comparison of the permeate chromate concentration for the semiequilibrium dialysis cell and the spiral wound system is shown in Figure VI.19. A comparison of the percent rejection of the chromate for the spiral wound system and semiequilibrium dialysis cell is shown in Figure VI.20. 


\subsection{Discussion}

\subsubsection{Flux for Spiral-Wound Membrane}

Fluxes were studied at four different outlet retentate flow rates using CPC as the surfactant and TBP as the target organic. Relative flux is defined as flux divided by the flux of the pure solvent under the same conditions. As shown in Figures VI.1 and VI.4 both flux and relative flux decreased monotonically as surfactant concentration increased.

Increasing the linear velocity of flow across the surface of the membrane (increasing the outlet retentate flow rate) minimizes the laminar sub-layer thickness, reducing the effects of concentration polarization as illustrated in Figure II.4. The reduction of the thickness of the laminar sub-layer region increases the concentration gradient across the region, thus increasing the molar flux of rejected species away from the membrane surface to the bulk solution. This decreases the concentration of rejected species near the membrane surface, thus increasing the permeate flux.

There is not an appreciable increase in the permeate flux by increasing the outlet retentate flowrate from $1600 \mathrm{~mL}$ per minute to 3400 $\mathrm{mL}$ per minute. The permeate concentrations of TBP and CPC decrease with increasing outlet retentate flowrate for a given average retentate concentration as shown in Figure VI.2 and Figure VI.3. The $1600 \mathrm{~mL}$ per minute and $3440 \mathrm{~mL}$ per minute outlet retentate flowrates resulted in the minimum permeate concentrations. The lower permeate concentrations of 
CPC and TBP are probably the result of reduced concentrations of rejected species at the membrane surface. However, there does not appear to be a significant reduction of the concentration polarization effect by increasing the outlet retentate flowrate above $1600 \mathrm{~mL}$ per minute. After considering the flux results in Figure VI.1 and the permeate concentration values in Figure VI.2 and VI.3, all subsequent experiments were performed at a outlet retentate flowrate of $1600 \mathrm{~mL}$ per minute.

The gel concentration or gel point is the retentate concentration at which permeate flux becomes zero. From Figures VI.1 and VI.4. the gel concentration in Figure VI.1 is near an average retentate CPC concentration of $600 \mathrm{mM}$ and does not change with changes in the outlet retentate flowrate. The gel concentration, near $600 \mathrm{mM}$, in Figure VI.4 is nearly the same for both the stirred cell and the spiral wound membrane.

\subsubsection{Comparison of Stirred Cell and Spiral Wound Relative Flux}

Previous stirred cell work has shown that the relative permeate flux and surfactant permeate concentration are dependant on stirrer speed or the linear velocity of the retentate solution near the membrane and that their is an optimum stirrer speed for the stirred cell (62) just as there is and optimum linear flow rate for the spiral wound. The comparison of flux for stirred cell (from 34) and spiral wound 5,000 MWCO membranes (this work) is shown in Figure VI.4. The permeate flux for the spiral wound membrane compares very well with the stirred cell permeate flux on a per 
unit area basis, especially considering that membrane surface area for the stirred cell is at least two orders of magnitude smaller than the spiral wound membrane surface area. A comparison of Figure VI.1 with Figure VI.4 shows a striking similarity in the curve shapes. The difference in permeate flux values are more prominent in the midrange retentate concentrations. The stirred cell has a severe retentate linear velocity gradient ranging from zero directly in the center of the membrane to a maximum velocity at the outer edges of the membrane as illustrated in Figure II.6. In contrast, the linear retentate velocity in the spiral wound unit varies only moderately between the inlet and outlet.

\subsubsection{Comparison of Stirred Cell and Spiral Wound Permeate Concentrations for the 5,000 MWCO Membrane}

The permeate concentrations shown in Figure VI.5 and VI.6 for the stirred cell and the spiral wound membrane are very similar. The stirred cell has slightly lower permeate CPC and TBP concentrations when the retentate CPC concentration is above $200 \mathrm{mM}$ while the spiral wound membrane has a slightly lower permeate CPC concentration below $90 \mathrm{mM}$ retentate CPC concentration. Overall, the performance (flux and permeate purity) of the stirred cell and spiral wound membranes are very similar and suggest that data collected from the stirred cell is adequate for the initial design and evaluation of an MEUF process for application to a industrial process stream when the target solute is a dissolved nonionic organic. 


\subsubsection{Comparison of SDS, CPC and TBP Permeate}

Concentrations for Different Spiral Wound Molecular .

Weight Cut Off Membranes

The permeate concentrations of CPC and TBP in Figure VI.7 and Figure VI.8 for the spiral wound unit follow the same general trend observed by Dunn. for a stirred cell of increasing permeate concentrations with increasing MWCO (62). However, Dunn did observe a minimum for the permeate CPC concentrations for the 10,000 MWCO membrane (62) which was not observed for the spiral wound results. The spiral wound permeate TBP concentrations increased with increasing membrane MWCO while Dunn observed smaller changes in permeate TBP concentrations with changing membrane MWCO (62). One can speculate that the screen next to the surface of the membrane in the spiral wound configuration is more effective at suppressing the development of the laminar sub-layer region than the stirring mechanism in the stirred cell. A thinner laminar sublayer would be characteristic of enhanced mixing near the surface and less presieving. The reduced presieving may be sausing the increasing TBP concentrations with increasing MWCO membranes observed using the spiral wound configuration.

Figure VI.9 and Figure VI.10 show permeate concentrations of SDS and TBP. Changing spiral wound membrane MWCO does not effect the permeate concentration of TBP as Dunn observed for the CPC/TBP system (62). There is also a minimum for the permeate SDS concentrations for the 
20,000 MWCO spiral wound membrane like there was for the Dunn 10,000 MWCO stirred cell observations (62).

\subsubsection{The Effect of Added Electrolyte on Surfactant Permeate Concentration}

The effect of added electrolyte (target metals) on the surfactant permeate concentration is shown in Figure VI.11 through Figure VI.13. The ratio of feed (retentate inlet) surfactant concentration to metal concentration for the experiments done here is shown in Figure VI.14 through Figure VI.16. In general, the permeate surfactant concentration decreases as the target metal concentration increases, as is illustrated by Figures VI.11 and VI.13 which shows two different experiments each with different surfactant to target metal ratios as shown in Figure VI.14 and VI.16, respectively. The ratio of surfactant concentration to metal concentration for the feed remains constant for the duration of the experiment even though additional surfactant/metal solution concentrate is incrementally added to the system and permeate solution is incrementally removed from the system in order to concentrate the surfactant and metal

remaining in the system. In Figure VI.14, the high feed ratio of 0.16 leveling off to 0.1 was due to inadequate mixing of the surfactant/metal solution in a tank from which the surfactant/metal solution is routed to the MEUF system. However, the higher metal concentration to surfactant concentration ratio does illustrate the effect added electrolyte has on the 
surfactant CMC of anionic surfactants and therefore, on permeate surfactant concentrations. The permeate SDS concentrations shown in Figure VI.II illustrates that an increase in the ratio of metal concentration to surfactant concentration decreases the permeate SDS concentration. The permeate SDS and CPC concentrations for experiments with added electrolyte are all significantly below their respective pure surfactant solution CMC values of $0.8 \mathrm{mM}$ and $8.2 \mathrm{mM}$ over a large range of average surfactant retentate concentrations. These results are not unexpected since the CMC of an ionic surfactant solution is depressed by added electrolyte (16). The depression of the CMC means the monomer concentration of the ionic surfactant is reduced and therefore, the equilibrium surfactant monomer concentration in the permeate is also reduced.

\subsubsection{Spiral Wound and Semiequilibrium Dialysis Systems and Metal Ions and Metal Complexes.}

The spiral wound membrane permeate copper concentrations are shown in Figures VI.17, and the rejection of of copper is shown in Figure VI.18. The percent rejection ranges between 99 and 100 percent and shows that MEUF can concentrate target metals such as copper very effectively. There is one set of spiral wound membrane data that was collected at a copper to surfactant concentration ratio greater than one to ten and is shown in Figure VI.17. The different feed copper to surfactant concentration ratios affected the permeate concentrations as expected, 
increasing the copper to surfactant concetration ratio increases the permeate copper concentration at a given surfactant feed concentration.

Figures VI.19 and Figure VI.20 show a comparison of spiral wound MEUF and semiequilibrium dialysis permeate chromate concentration and chormate rejection. The semiequilibrium dialysis permeate chromate concentration is approximately one half the permeate chromate concentration observed for the spiral wound chromate permeate.

\subsection{Conclusions}

MEUF has been shown to effectively remove either a dissolved organic solute or divalent cationic heavy metal in a spiral wound membrane with high flux and rejection until high retentate surfactant concentrations are attained.

A comparison of results from stirred cell, semiequilibrium dialysis and spiral wound stucies validates the use of stirred cell and semiequilibrium data to do initial design and evaluation of the application of MEUF for an industrial process stream containing a nonionic organic pollutant. 


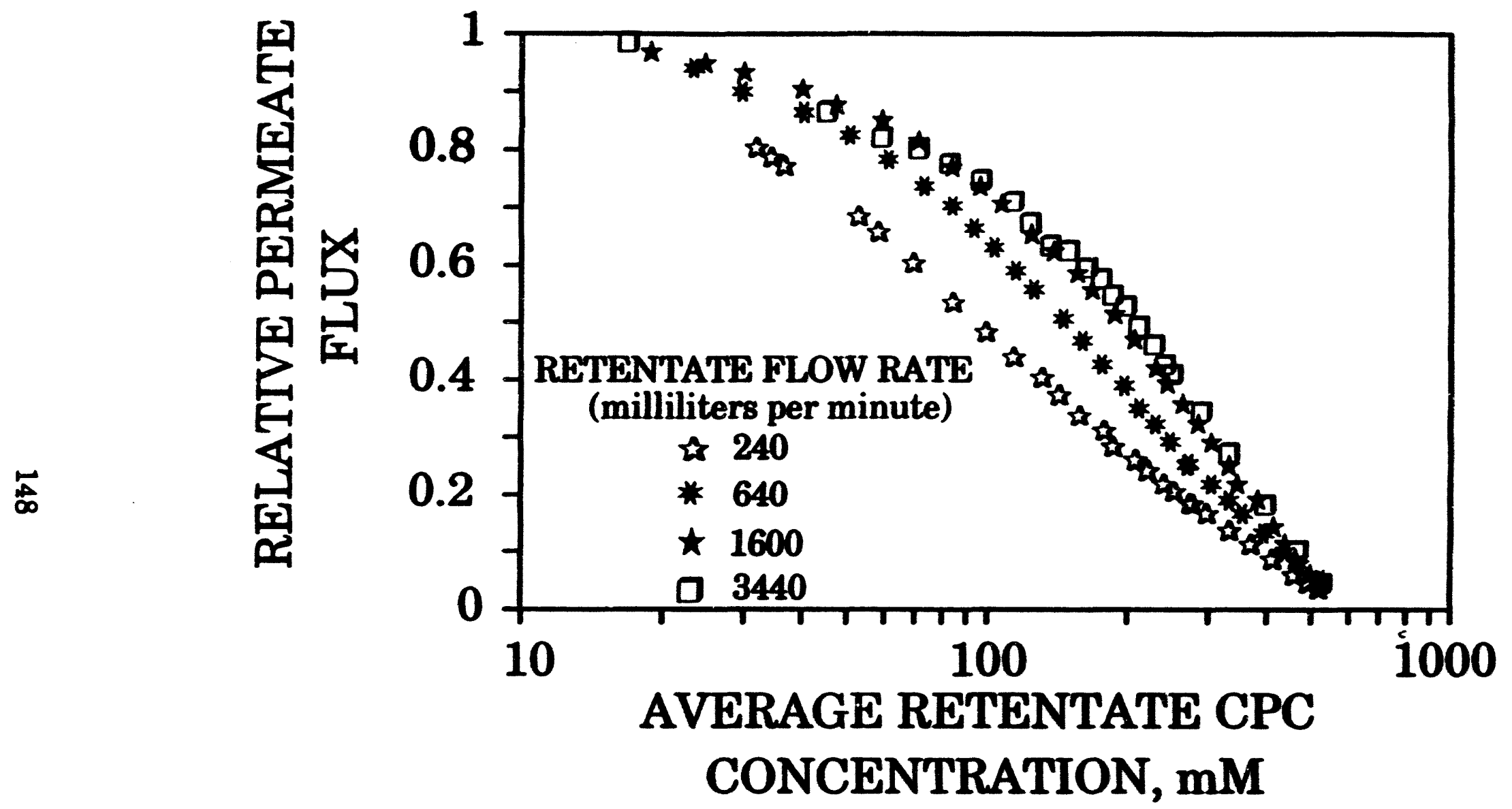

FIGURE VI.1: . Relative Permeate Flux Using CPC and 5,000 MWCO

Spiral Wound Membrane 


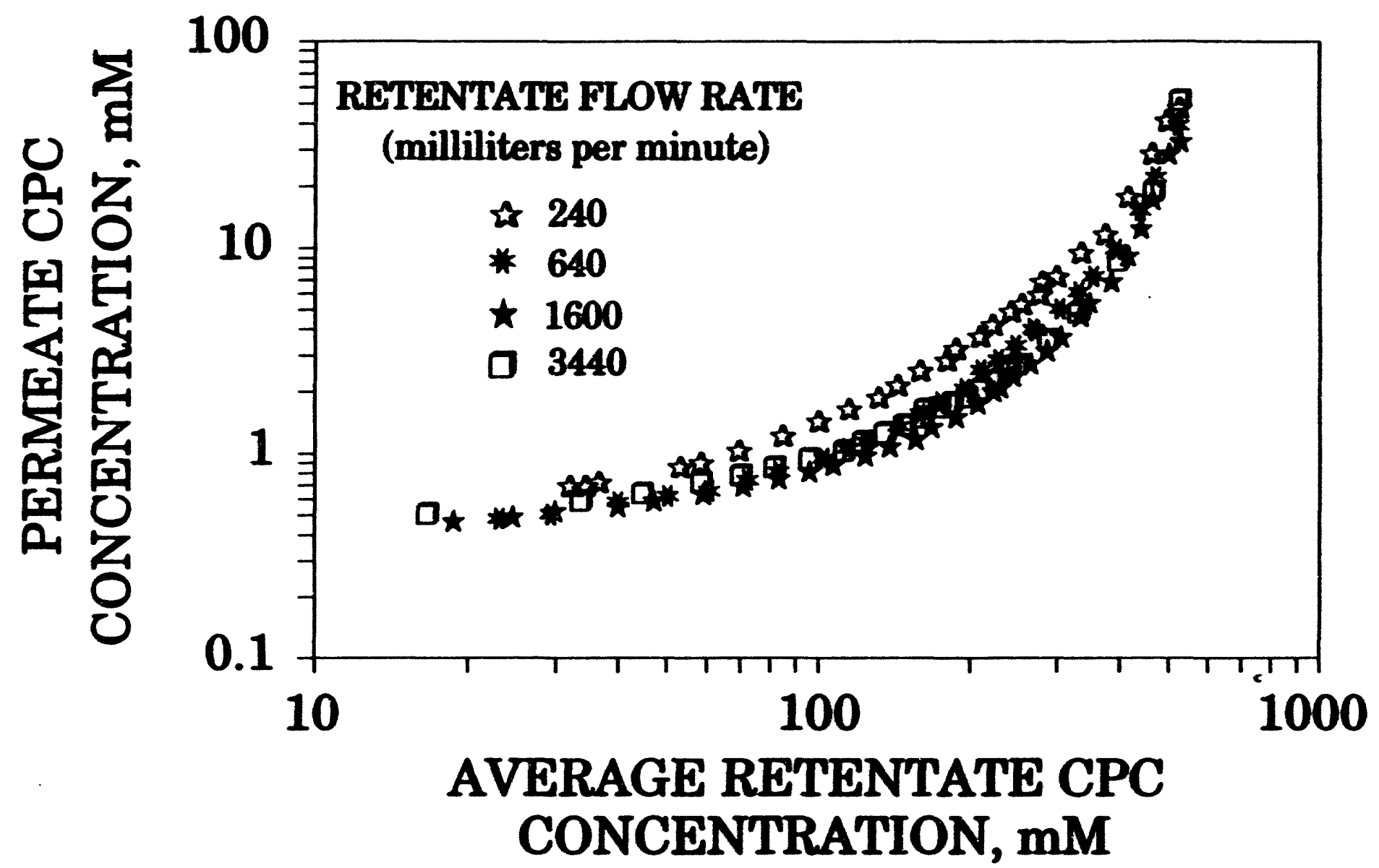

FIGURE VI.2: Permeate CPC Concentrations Using CPC and 5,C00 MWCO Spiral Wound Membrane 


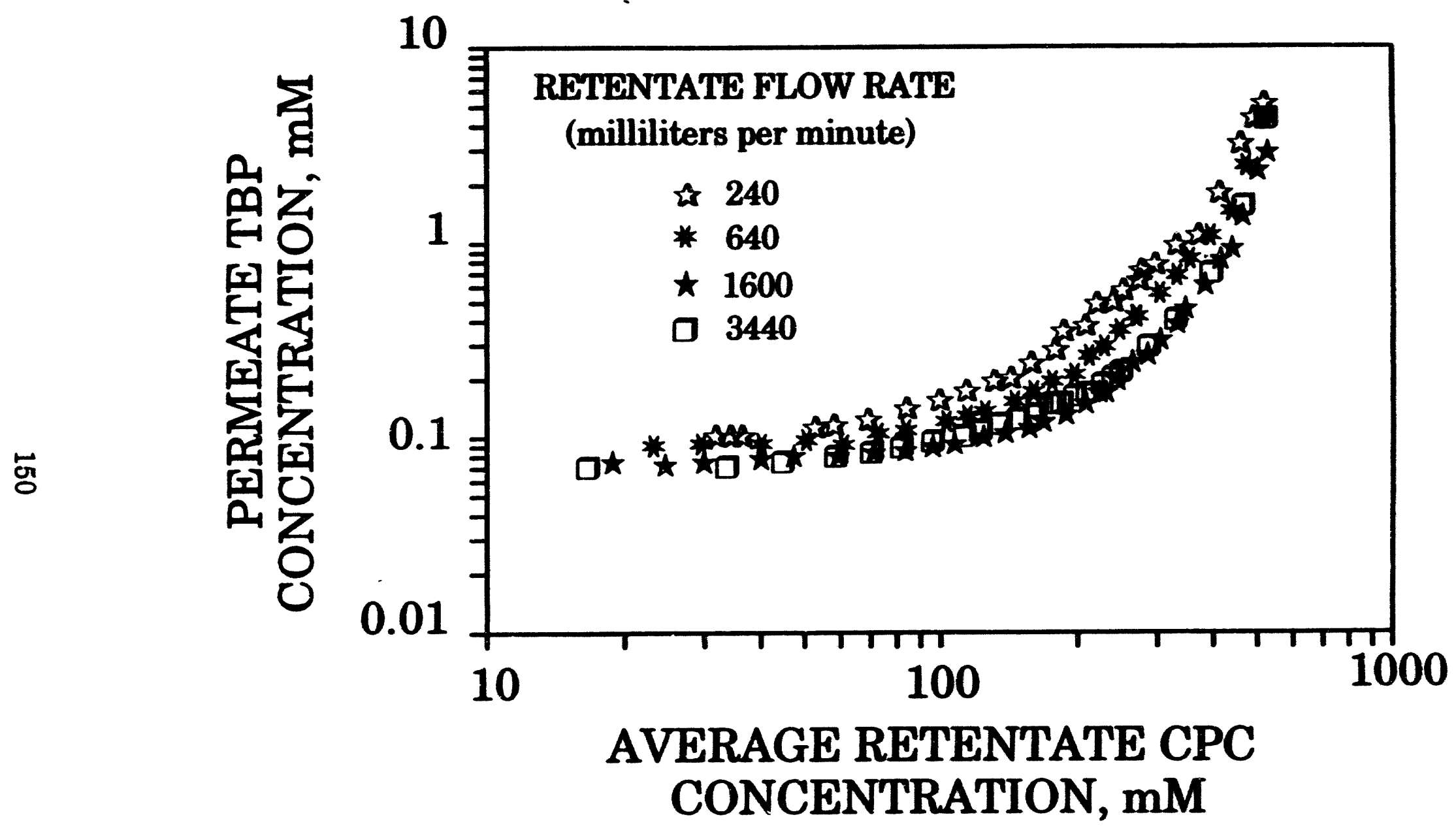

FIGURE VI.3: Permeate TBP Concentration Using CPC and 5,000 MWCO Spiral Wound Membrane 


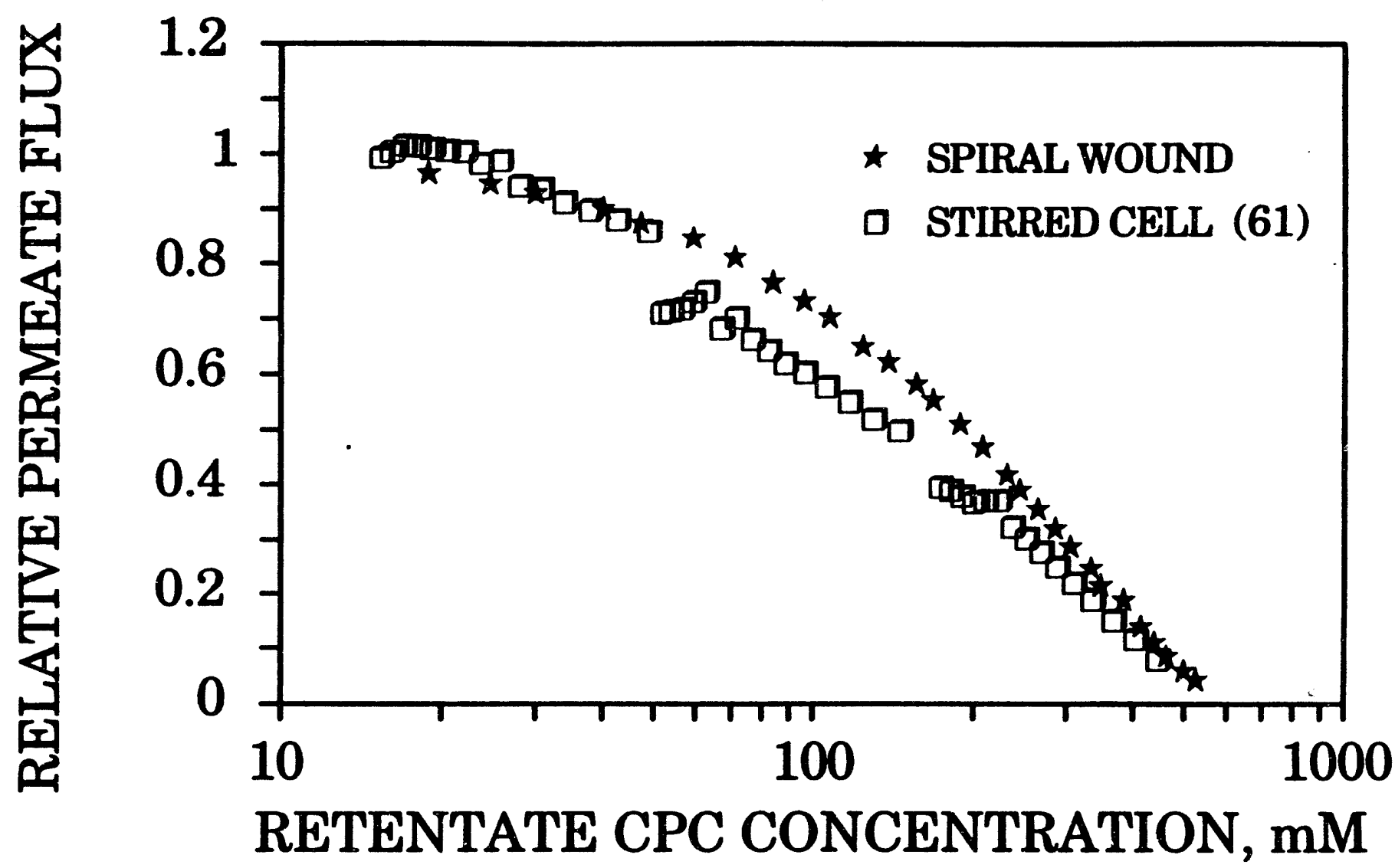

FIGURE VI.4: Comparison of Stirred Cell and Spiral Wound Membrane Relative Permeate Fluxes for a 5,000 MWCO Membrane 


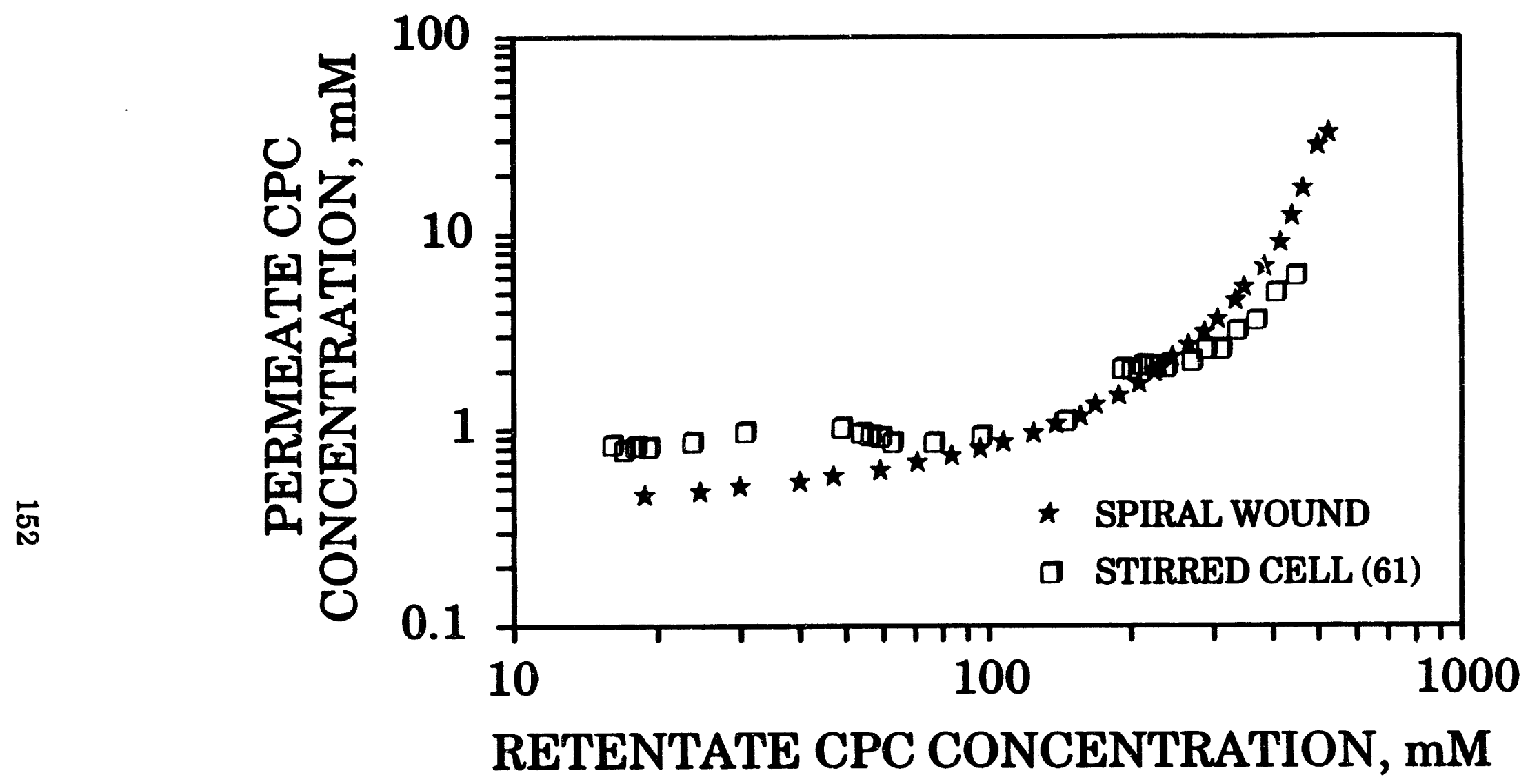

FIGURE VI.5: Comparison of Stirred Cell and Spiral Wound Ultrafiltration Membrane Performance, CPC, 5,000 MWCO Membrane 


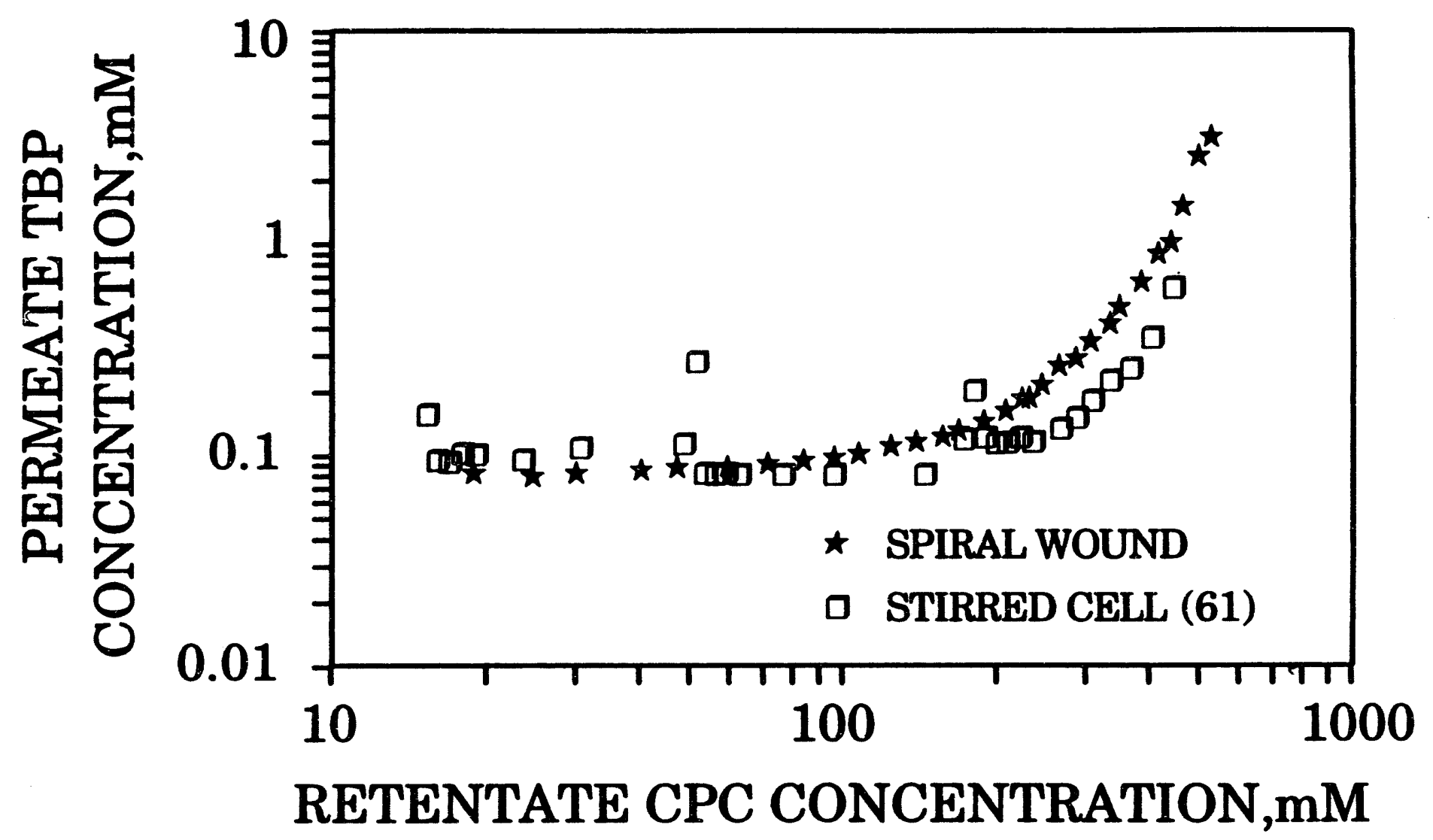

FIGURE VI.6: Comparison of Stirred Cell and Spiral Wound Membrane Performance, TBP, 5,000 MWCO Membrane 


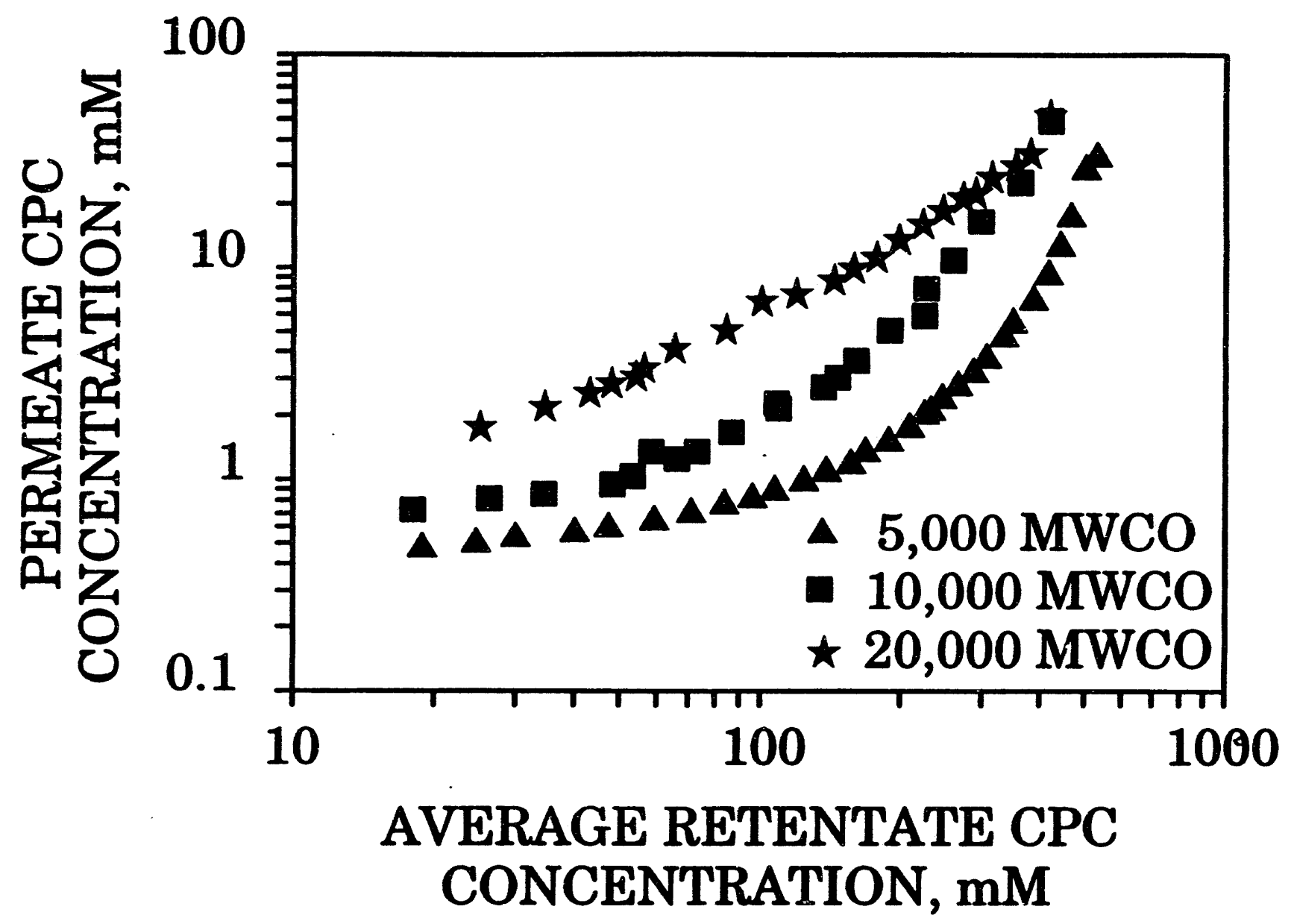

FIGURE VI.7: Comparison of CPC Permeate Concentration for Different Molecular Weight Cut Off Spiral Wound Membranes 


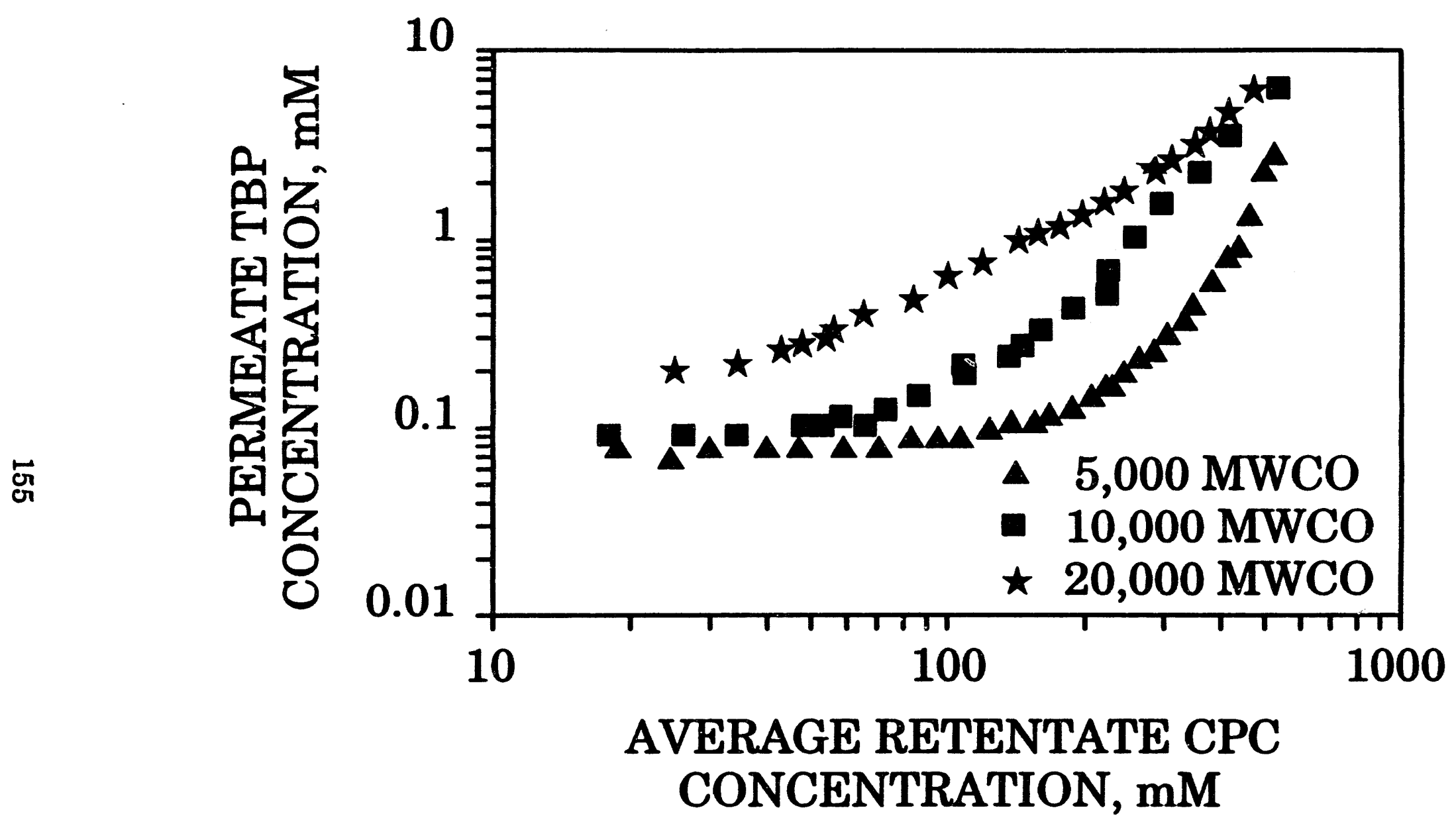

FIGURE VI.8: Comparison of TBP Concentrations for Different Molecular Weight Cut Off Spiral Wound Membranes 


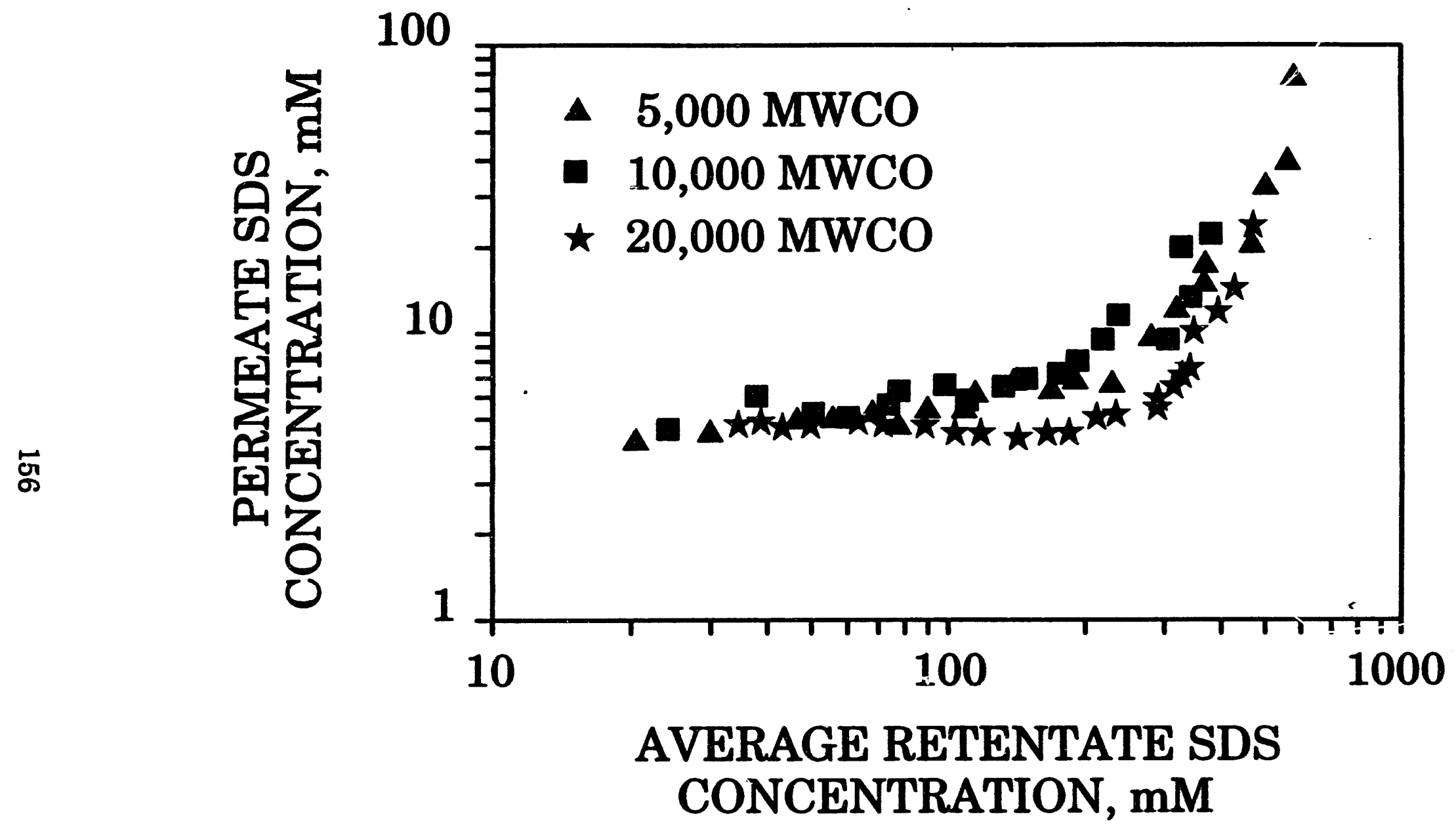

FIGURE VI.9: Comparison of SDS Permeate Concentration for Different Molecular Weight Cut Off Spiral Wound Membranes 


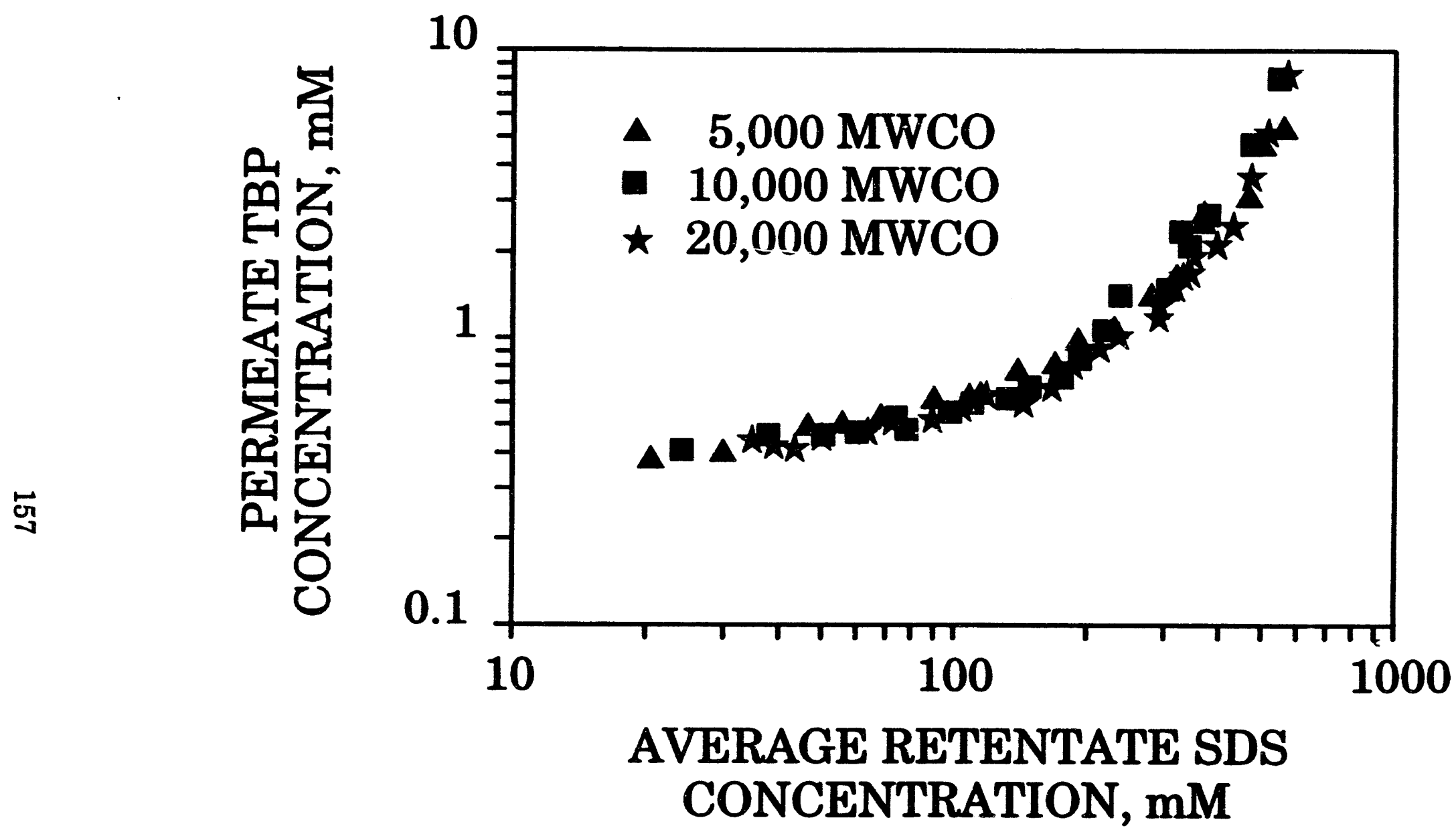

FIGURE VI.10: Comparison of TBP Permeate Concentration for Different Molecular Weight Cut Off Spiral Wound Membranes 


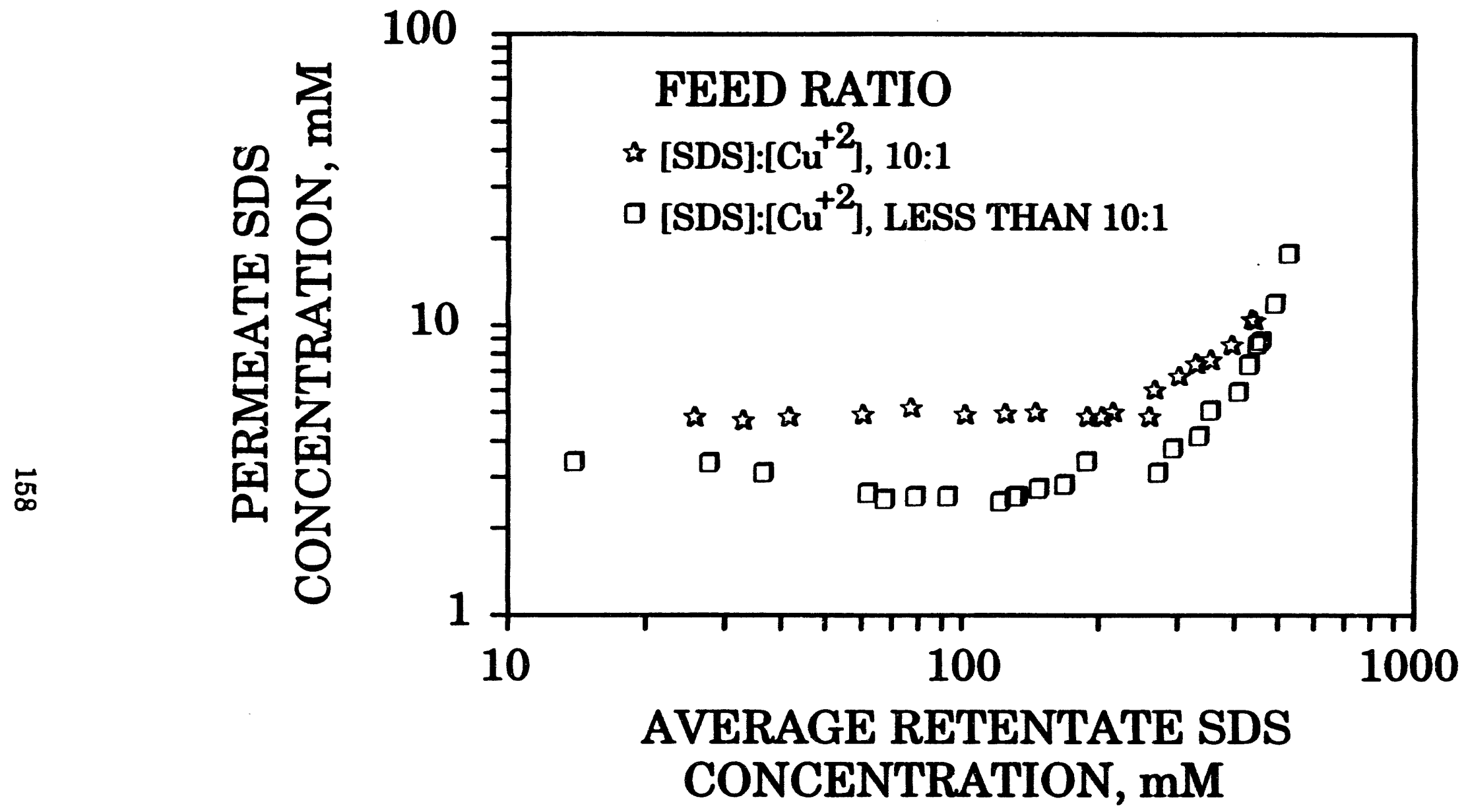

FIGURE VI.11: The Comparison of the SDS Permeate Concentrations at Different Copper Feed Concentrations for a 10,000 MWCO Spiral Wound Membrane 


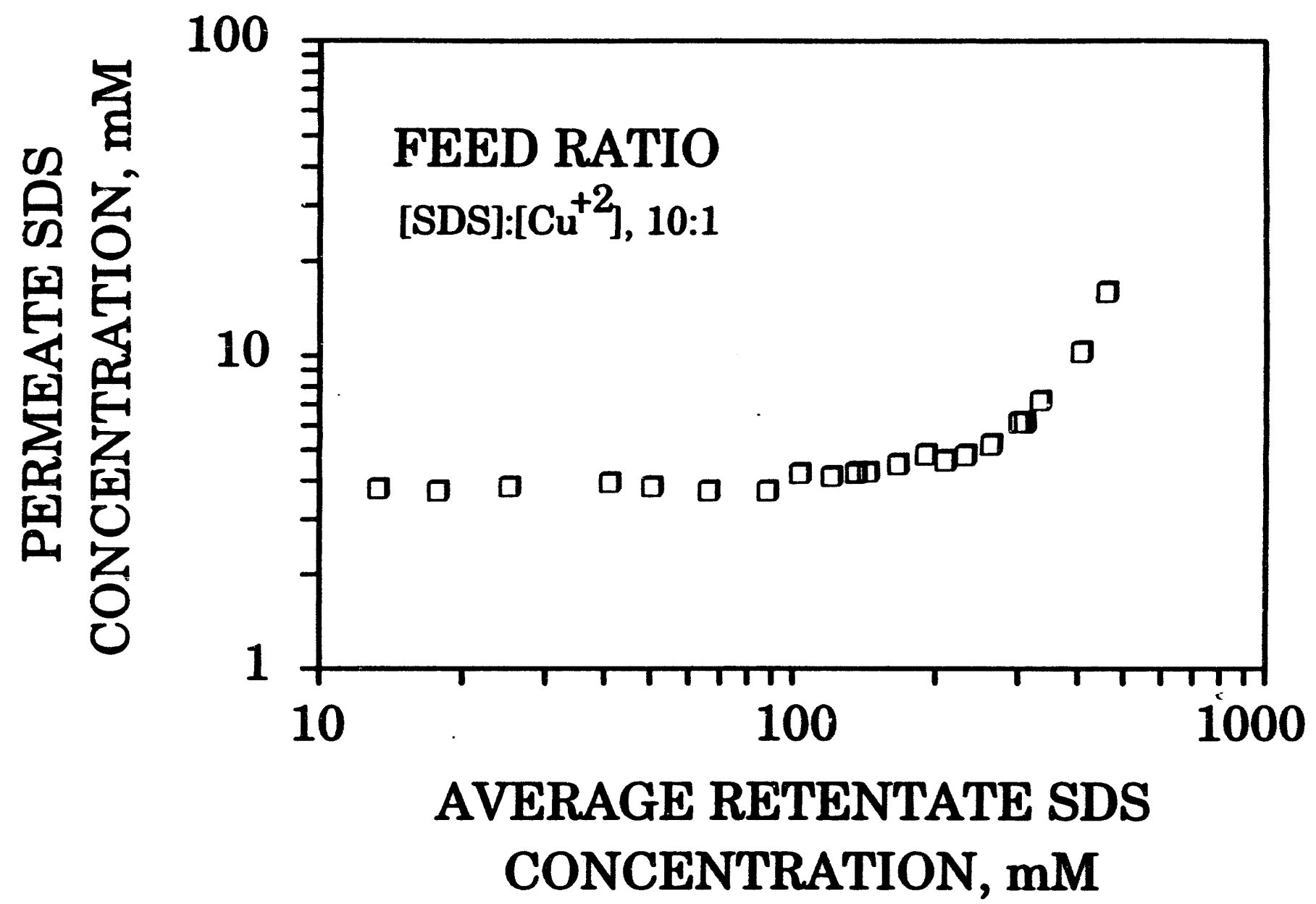

FIGURE VI.12: SDS Permeate Concentration for Retentate Solutions Composed of TBP and $\mathrm{Cu}^{+2}$ and Using a Spiral Wound 20,000 MWCO Membrane 


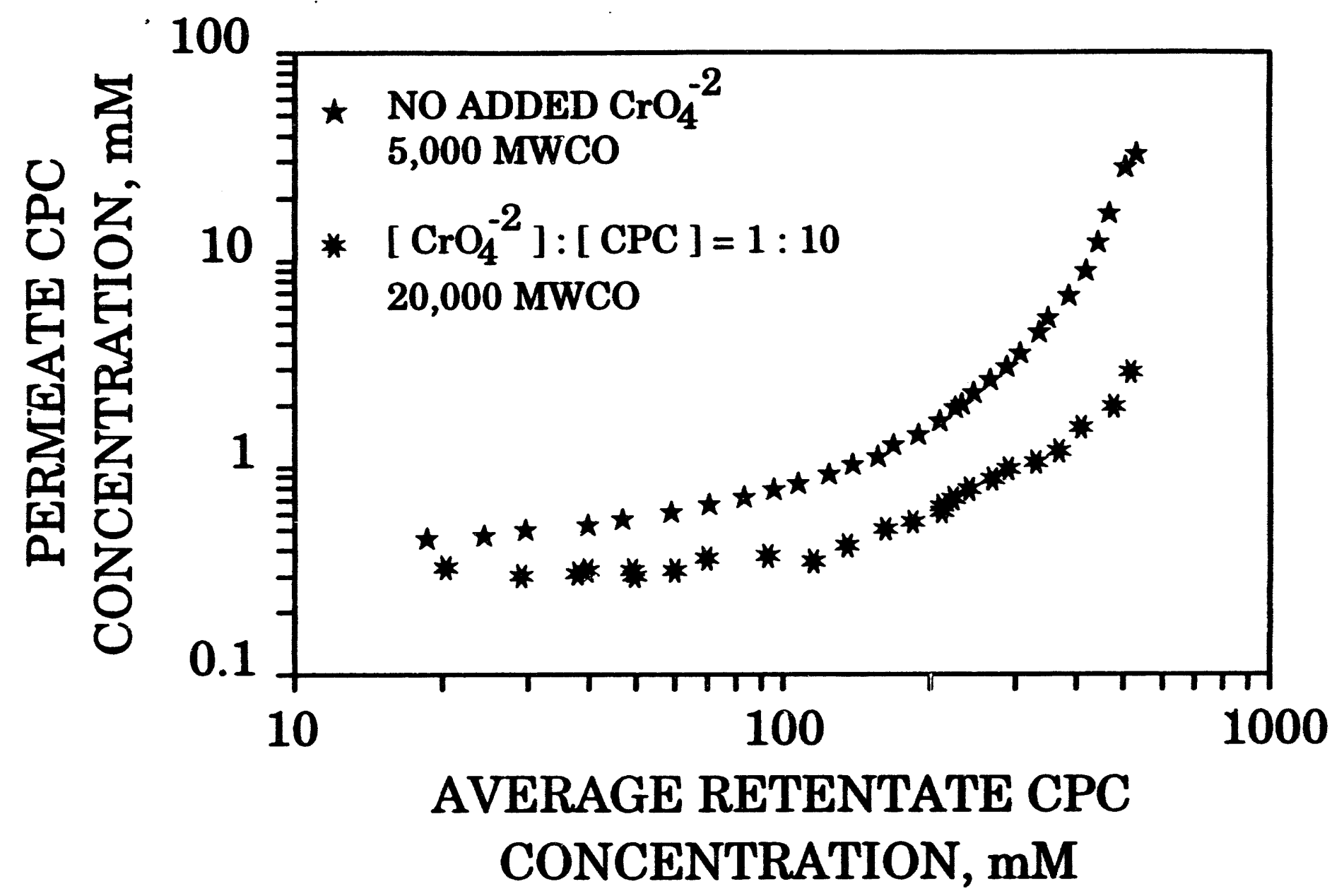

FIGURE VI.13: Permeate Concentration of CPC with and without $\mathrm{Added}_{\mathrm{CrO}_{4}}{ }^{-2}$ Using a 5,000 MWCO and a 20,000 MWCO Spiral Wound Membrane 


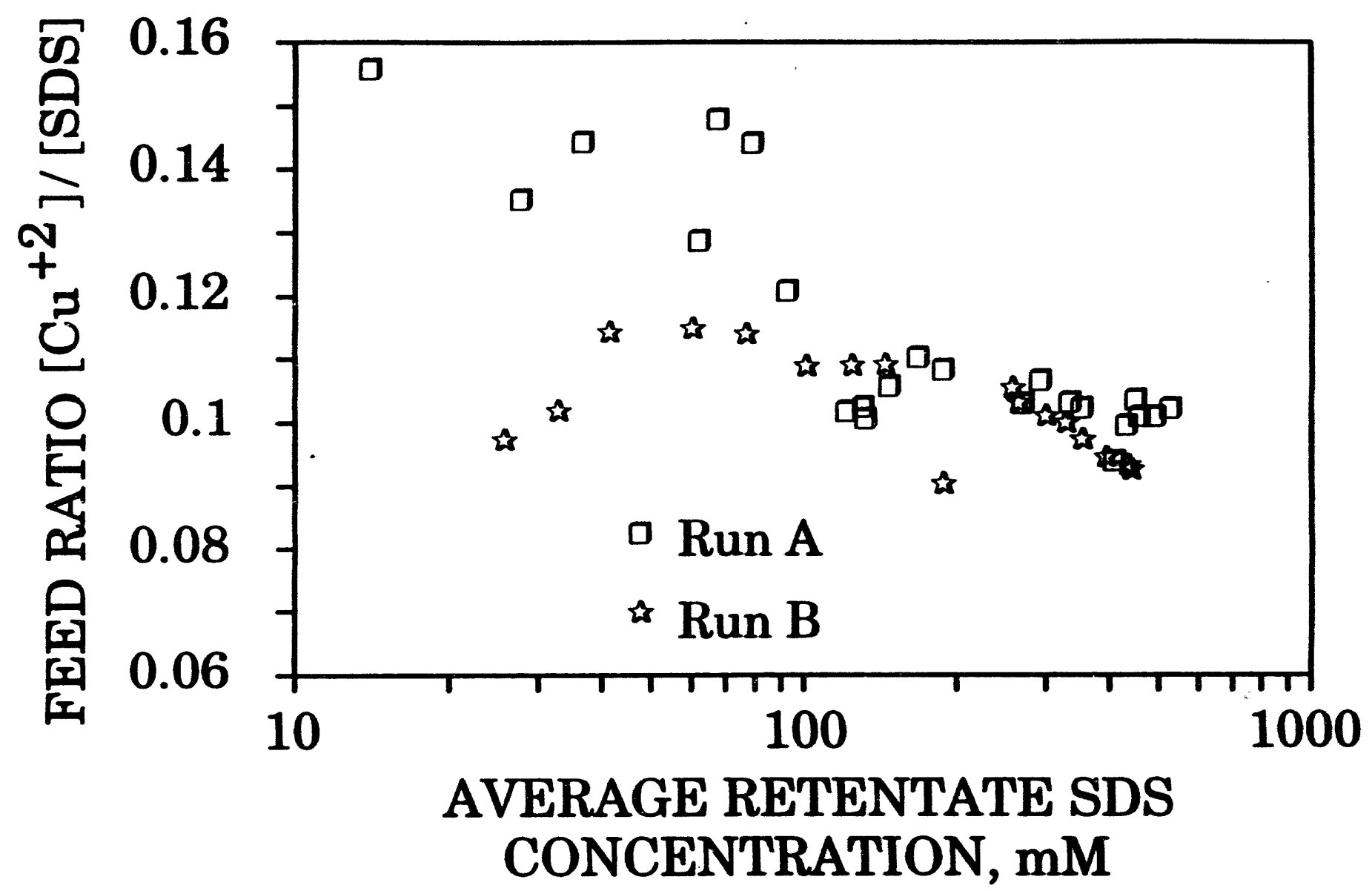

FIGURE VI.14: Ratio of $\mathrm{Cu}^{+2}$ Concentration to SDS Concentration for the 10,000 MWCO Spiral Wound Membrane Study 


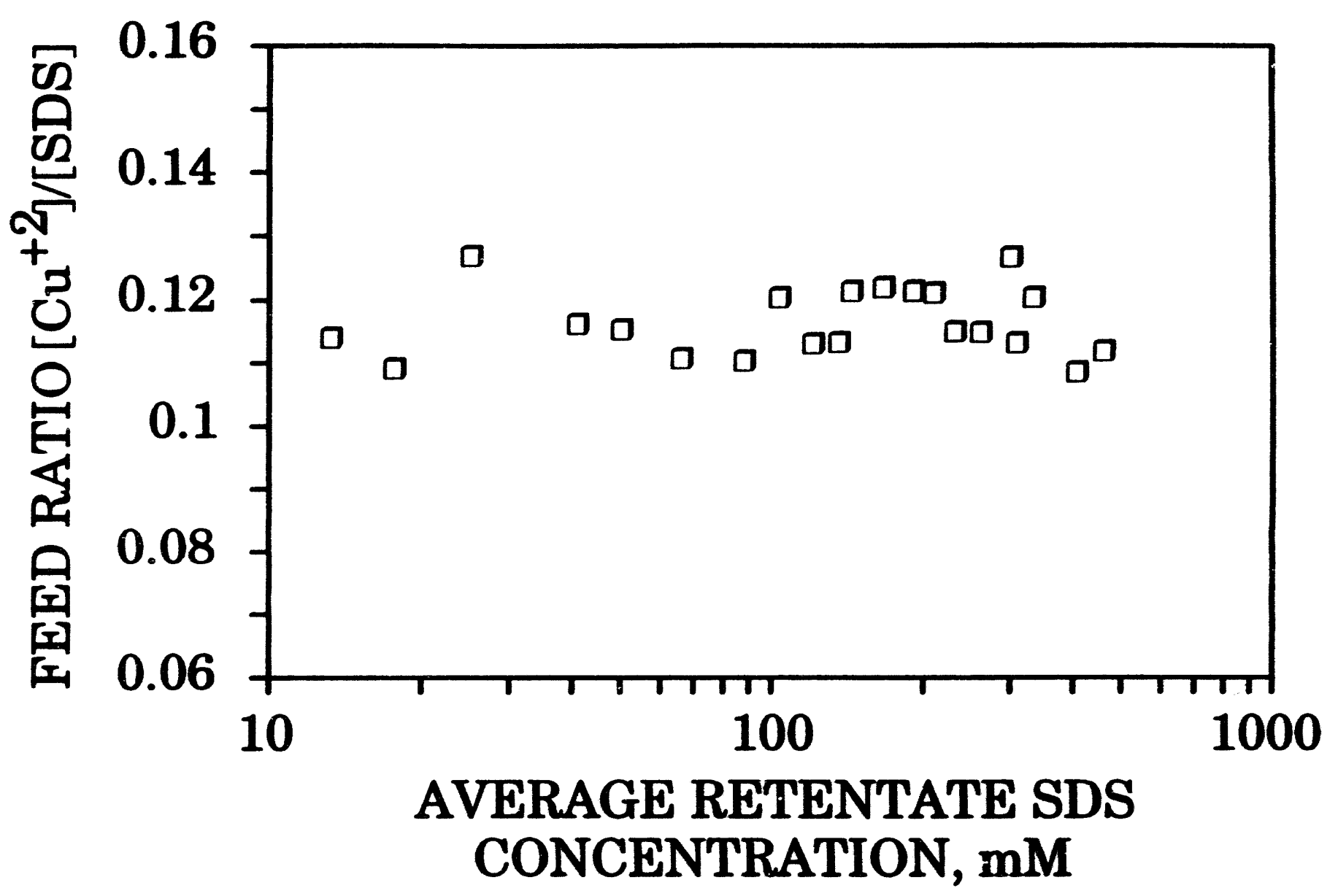

FIGURE VI.15: Ratio of $\mathrm{Cu}^{+2}$ Concentration to SDS Concentration for the 20,000 MWCO Spiral Wound Membrane Study 


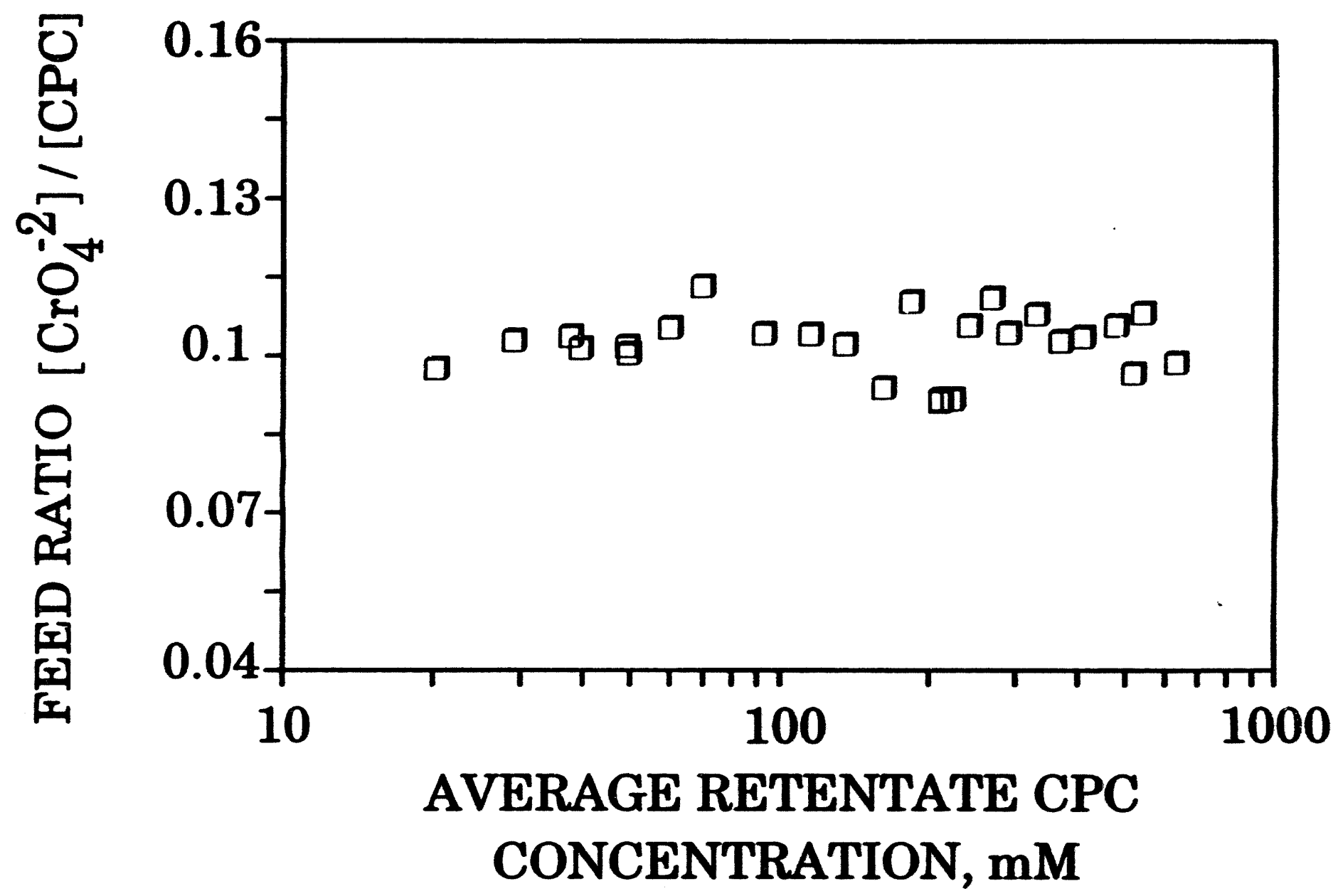

FIGURE VI.16: Ratio of $\mathrm{CrO}_{4}^{-2}$ Concentration to CPC Concentration for the 20,000 MWCO Spiral Wound Membrane Study 


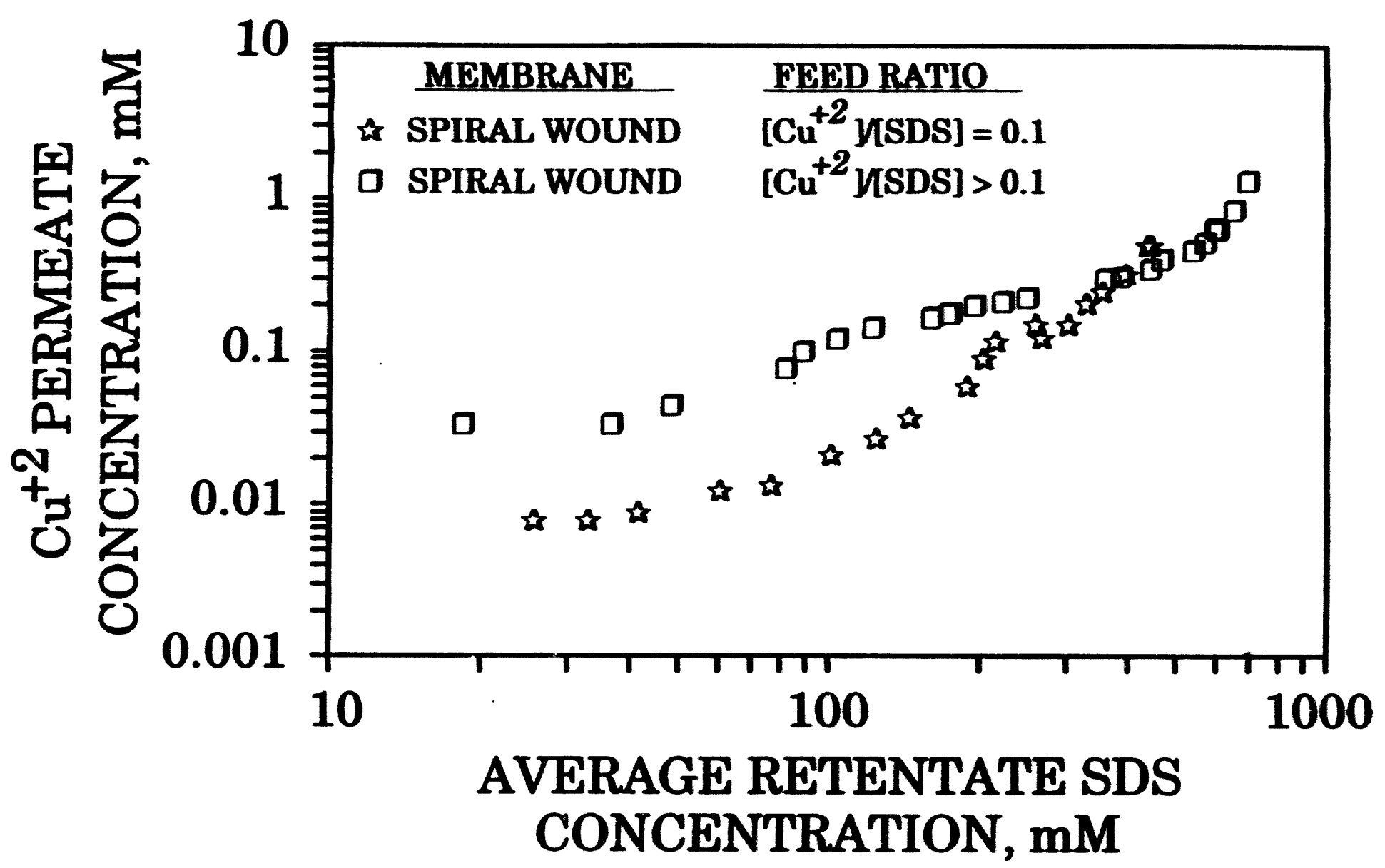

FIGURE VI.17: Permeate $\mathrm{Cu}^{+2}$ Concentration for a Spiral Wound Membrane, 10,000 MWCO 


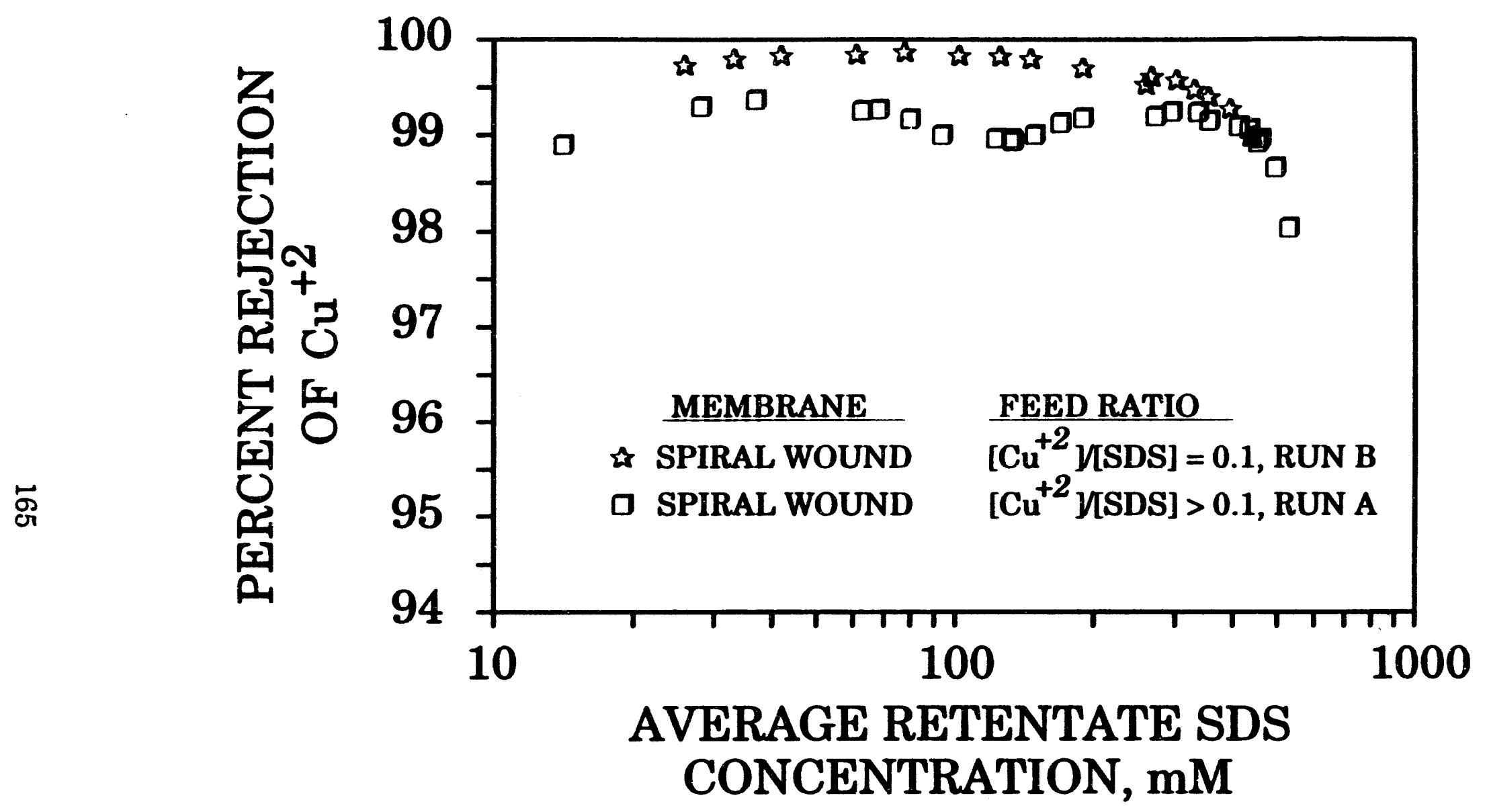

FIGURE VI.18: Percent Rejection of $\mathrm{Cu}^{+2}$ for Spiral Wound 10,000 MWCO Membranes 


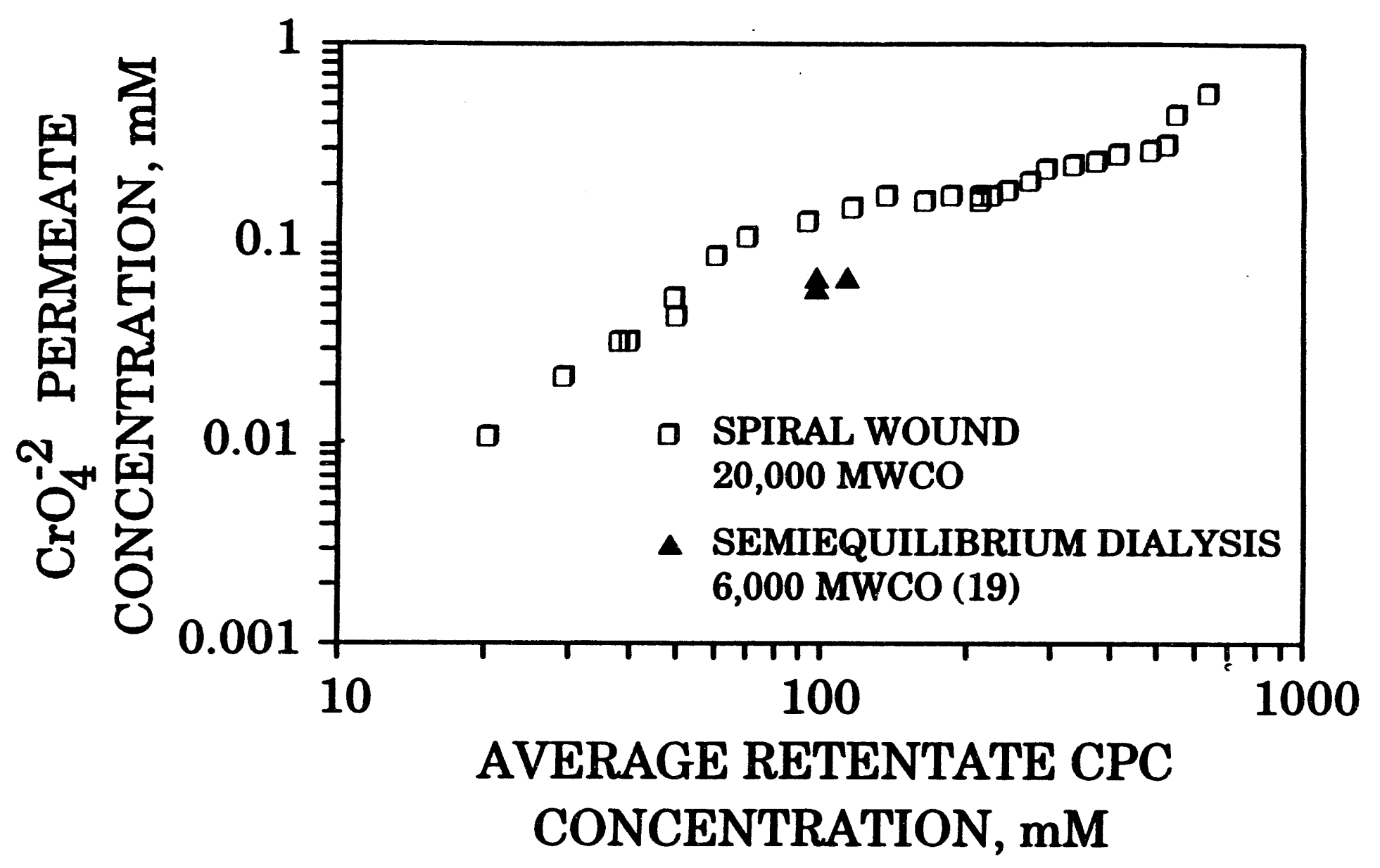

FIGURE VI.19: Comparison of the Permeate $\mathrm{CrO}_{4}^{-2}$ Concentration for a Semiequilibrium Dialysis Cell and Spiral Wound Membrane 


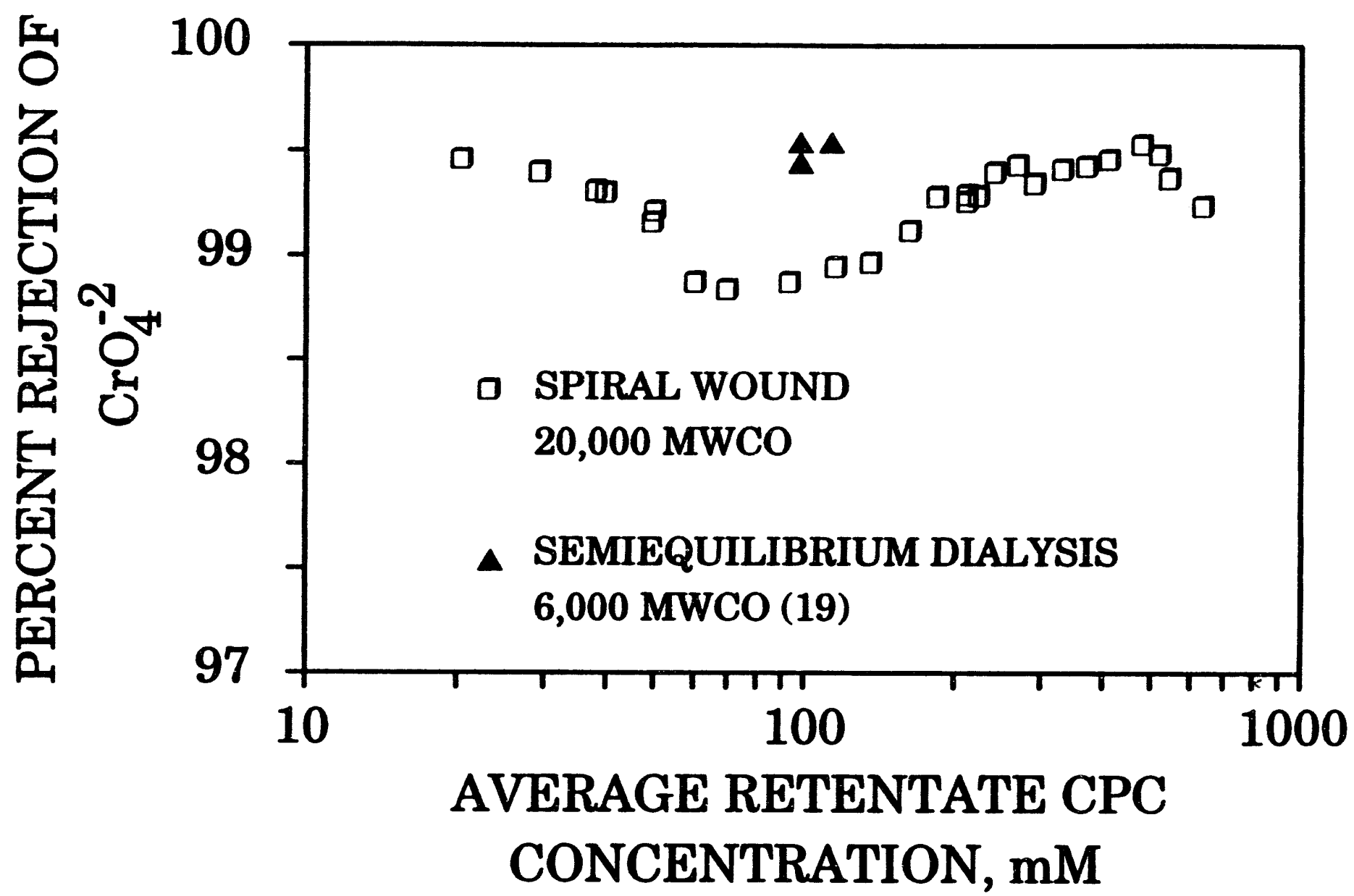

FIGURE VI.20: Comparison of the Percent Rejection of $\mathrm{CrO}_{4}^{-2}$ for Spiral Wound and Semiequilibrium Dialysis Membranes 


\section{CHAPTER 7}

\section{EVALUATION OF THE APPLICATION OF MEUF FOR THE' RECOVERY OF VOLATILE CHLORINATED HYDROCARBON COMPOUNDS FROM GROUNDWATER}

\subsection{Introduction}

The purpose of this study is to examine the feasibility of a new separation process, micellar enhanced ultrafiltration, to remove $99+$ percent of the volatile chloronated hydrocarbon compounds ( $\mathrm{CHC}$ ) from polluted water.

Micellar-enhanced ultrafiltration (MEUF) is a novel separation technique which provides the opportunity to remove dissolved organics from wastewater in a process which is potentially much more efficient than alternative methods such as activated carbon adsorption.

In addition to pollution control, there are critical needs for concentration and purification of solutes from water in the production of important industrial materials. A prime example is the concentration of valuable biochemicals from fermentation broth.

This study involves the experimental investigation of the MEUF process and a downstream vacuum stripping operation for removing the CHC from water. As a result of these investigations for several surfactants, a surfactant of choice is selected and design parameters generated for that system. Design calculations are then performed to estimate the performance of the vacuum stripper and the MEUF units in a variable number of stages in a commercial process. Finally, based on these feasibility studies, 
preliminary economic estimates of the cost of applying this technology are made.

\subsection{Process Flow Diagram to Remove CHC from Water}

The use of MEUF to remove 99+ percent of CHC from contaminated groundwater is anticipated to require multiple stages as will be shown. In each MEUF stage, the retentate will be vacuum stripped to separate the CHC (as overheads from the stripping column) from the surfactant (removed as bottoms from the stripping column in a concentrated solution). Therefore each stage consists of a MEUF unit and a vacuum stripping column. A four-stage process flow diagram is shown in Figure VII.1. An alternative configuration is shown in Figure VII.2, where all the retentates from each of the four MEUF stages are fed to a central vacuum stripper. The concentrated surfactant solution from the stripper is recycled back to each MEUF stage. In this study, we have shown that either configuration is technically feasible - more detailed studies and economic calculations are necessary to determine the most economical system.

The CHC and water from the stripper overheads are condensed after leaving the vacuum pump discharge. The resultant waste stream will ordinarily be a CHC phase and a water phase saturated with CHC. However, for a very dilute groundwater source stream, the $\mathrm{CHC}$ concentration may not be great enough to form a separate phase. The water phase in either case can be treated by traditional methods such as biological degradation techniques or can be recycled to a stripper from the 
first stage. In this latter case, the only effluent from the process will be a very small volume of a mixture of CHC. For example, in treating 100,000 gallons of water with $1 \mathrm{ppm}$ TCE, the resulting TCE stream would be approximately one pound.

Even though the surfactant concentration in the ultimate permeate stream from stage 4 is very low, the economics of the operation can be improved by treating the permeate to recover the surfactant. We will show data for one method of treating this stream (use of a low MWCO ultrafiltration membrane). Other possible solutions should be studied in future research. A small make-up surfactant stream is added to the feed entering stage 1 to replace the surfactant lost in the permeate from stage 4 . This make-up stream could be recovered surfactant from the final permeate, or fresh surfactant, or a combination of the two.

The effluent from this process is (1) a water stream containing almost all of the fresh feed water and less than 1 percent of the $\mathrm{CHC}$ in the fresh feed, (2) a liquid CHC stream for reuse or disposal - this stream will have a very small volume for the $\mathrm{CHC}$ concentrations in the feed of interest here, and (3) potentially a water stream containing a high concentration of CHC, perhaps at saturation. If the stream is recycled to the stage 1 stripper, this will not be a process effluent, and in any case, it will be substantially less than 1 percent of the volume of the fresh feed groundwater. 


\subsection{Results and Discussion}

The results of this study will be discussed as follows: measurement of the separation efficiency and flux of the MEUF step for each stage - this also includes surfactant recovery from the final permeate stream and selection of the optimum surfactant in this system, (2) measurement and modeling of the stripping step for each stage, (3) process design calculations to optimize the number of stages of the process, the stripping column operation, and to obtain a cost estimate for the process, and (4) a description of the experimental apparatus and techniques used here.

\subsubsection{Separation of TCE from Water in MEUF Units}

\subsubsection{General Considerations}

There are two primary parameters of interest in designing the ultrafiltration units: separation factor (CHC concentration ratio in retentate/permeate), and flux. The number of stages required to attain a separation increases as the separation factor decreases, and the membrane area needed and resulting capital cost increase as the flux decreases. A great deal of work in this project involved selection of a surfactant with acceptable properties.

It has been shown that flux behavior in MEUF tend to follow classical concentration polarization behavior: the flux vs. log (retentate 
concentration) is linear over a wide range of flux values (4.21.24). For comparison, relative flux is often considered, where relative flux is flux/flux in pure solvent. The ultrafiltration can be performed in a given stage until an unacceptably small flux (generally around a relative flux of 0.1 or 0.2 ) is attained. This is critical because it dictates the permeate/feed ratio in a given stage. If a substantial increase in the concentration of surfactant and $\mathrm{CHC}$ in the retentate cannot be attained in a MEUF stage, the retentate volume which must be stripped becomes quite large and ultimately as much retentate could be stripped as the volume of the original feed stream. In such a case, one should simply vacuum strip the entire feed stream and not use MEUF. The whole advantage of using MEUF units is to reduce the volume of the streams which must be vacuum stripped to a level well below that of the fresh feed groundwater stream.

The higher the concentration of surfactant in the feed or the better a surfactant can solubilize the $\mathrm{CHC}$ per mole of surfactant, the more solubilization occurs and the lower the $\mathrm{CHC}$ concentration in the permeate from that stage - hence, the fewer the number of stages to attain the 99 percent removal of $\mathrm{CHC}$ from the water. However, the higher the surfactant concentration to a MEUF stage, the less permeate volume can be produced before the retentate surfactant concentration attains a value too high for acceptable flux and this increases the volume of the retentate which must be stripped. Everything else being equal, the more stages in a system, the lower the total retentate stream volume which must be stripped (summed over all stages) since each stage does not have to attain such a high degree of separation for the overall system to attain a 99 percent removal. Hence, 
we can summarize the effects of some variables on the key variable - the sum of the volumes of the retentate streams which must be vacuum stripped divided by the volume of the fresh feed as shown in Table VII.1.

TABLE VII.1

SUMMARY OF THE EFFECTS OF SOME VARIABLES ON THE SUM OF THE RETENTATE STREAMS DIVIDED BY THE VOLUME OF FRESH FEED

\begin{tabular}{|c|c|}
\hline VARLABLE & $\begin{array}{c}\text { (RETENTATE VOLUME)/ } \\
\text { (FRESH FEED VOLUME) }\end{array}$ \\
\hline $\begin{array}{c}\text { Increasing MEUF Retentate } \\
\text { Effluent } \\
\text { Surfactant Concentration }\end{array}$ & DECREASES \\
\hline $\begin{array}{c}\text { Increasing Solubilization of } \\
\text { CHC in Surfactant }\end{array}$ & DECREASES \\
\hline Increasing Number of Stages & DECREASES \\
\hline
\end{tabular}

Selection of an appropriate surfactant can cause the increase in the ultimate retentate surfactant concentration (before unacceptably low flux is 
attained) and can cause an increase in $\mathrm{CHC}$ solubilization. Increasing the number of stages adds capital cost to the process.

\subsubsection{Flux}

In the original experimental plan, we anticipated using large molecular weight nonionic surfactants since they have extremely low CMC values so that very low surfactant concentration levels in the permeate would be expected (eliminating the necessity of a surfactant recovery from the permeate from the last MEUF stage). Nonionic surfactants also have the advantage of not precipitating from solution at low temperatures. Figure VII.3 shows relative flux for DNP-18 as a function of retentate surfactant concentration in a spiral wound ultrafiltration unit. At a retentate concentration of about $0.1 \mathrm{M}$, the relative flux is approximately 0.1. As will be shown in staging calculations, this an unacceptably low flux, so other surfactants were evaluated.

In considering ionic surfactants, several properties were defined which were necessary: a reasonable flux at high retentate concentrations; a high degree of solubilization of CHC; a low surfactant concentration in the permeate and/or properties which simplify recovery from the permeate from the last stage; a low Krafft temperature (temperature below which the surfactant precipitates). The surfactant selected was DOWFAX 8390. The relative flux is shown in Figure 5 for both spiral wound and stirred cell ultrafiltration devices. In a spiral wound device (the type anticipated for commercial use), a retentate concentration of $0.25 \mathrm{M}$ could be attained before the relative flux dropped to 0.1 . The DOWFAX 8390 has excellent 
resistance to precipitation with a $\mathrm{Krafft}$ temperature below $0^{\circ} \mathrm{C}$. It has a molecular weight of 642 and we will discuss later how it can be ultrafiltered from the ultimate permeate from the process with a very low MWCO membrane. The solubilization of TCE, PCE and TCEA will be shown to be excellent also. Therefore, DOWFAX 8390 was identified as the current surfactant of choice. Future research would involve more effort to improve the surfactant selection and the use of surfactant mixtures, but the performance of DOWFAX 8390 is quite acceptable. Figure VII.3 also shows the relative flux of DOWFAX 3B2, which is a lower molecular weight version of DOWFAX 8390. This showed a lower flux than the DOWFAX 8390 and was not considered further. A cationic surfactant, stearyl dimethyl benzyl ammonium chloride, was added to DOWFAX 8390 to test the anionic/cationic surfactant mixture. As shown in Figure VII.3, this system showed a better flux than the anionic surfactant alone, but it was not investigated further because there was concern about the tendency to precipitate at low temperatures. For example, the 10/90 cationic/anionic mole ratio mixture did not appear to precipitate at $0^{\circ} \mathrm{C}$, while a $20 / 80$ mixture did precipitate. The excellent flux observed in this system makes it a good candidate for study in a future research project, because the anionic/cationic surfactant mixture may allow much higher retentate concentrations under certain conditions.

In designing a unit based on the flux data shown in Figure VII.3, the membrane area per unit flow rate is found by integrating from the inlet feed composition to the effluent retentate composition and finding the total area required - the flux per unit membrane area at the beginning of the 
stage is greater than near the outlet where the retentate concentration is higher.

\subsubsection{Separation Efficiency}

In many past studies, it has been shown that the concentration of the organic solute in the permeate is the sarne as the unsolubilized solute concentration in the retentate $(65,66,63)$. Therefore, equilibrium solubilization data can be used to predict the separation factor or permeate solute concentration in MEUF. Since equilibrium solubilization data are much easier and faster to obtain than actual ultrafiltration data, this fact provides a way to screen surfactants and obtain trends rapidly. Ultrafiltration runs are necessary to confirm this condition for the actual system used and to obtain flux data.

The solubilization equilibrium constant in the limit as $[\mathrm{CHC}] \ll$ [micellar surfactant] is defined as follows:

$$
\mathrm{K}_{\mathrm{S}}=\frac{\cdot\left[\mathrm{CHC}_{\text {solubilized }}\right]}{\left[\mathrm{CHC}_{\text {unsolubilized }}\right]\left[\text { Surfactant }_{\text {micellar }}\right]}
$$

where the brackets denote concentrations. Since the unsolubilized CHC concentration is expected to be near to the permeate CHC concentration and (20) the micellar surfactant concentration is the total surfactant concentration in the retentate minus the CMC of the surfactant (which 
were measured or obtain from the literature), knowledge of $\mathrm{K}_{\mathrm{s}}$ allows prediction of permeate CHC concentration. The value of $\mathrm{K}_{\mathrm{s}}$ for $\mathrm{CHC}$ is obtained from head-space chromatography measurements, a traditional technique for measuring solubilization of volatile solutes. (64.65). The higher the value of $\mathrm{K}_{\mathrm{S}}$, the greater the extent of solubilization and the lower the CHC concentration in the permeate. Later in this chapter, the effect of $\mathrm{K}_{\mathrm{s}}$ on the design in a sensitivity analysis in material balance calculations will be examined. Later in this section, we will show data confirming that the permeate CHC concentration is equal to the unsolubilized concentration in the retentate for the optimum surfactant found in this study.

As a part of the search for a good surfactant candidate, undertaken because the flux of the DNP-18 system was found to be unacceptable, $K_{s}$ was measured for TCE, PCE and TCEA in a number of surfactant systems as seen in Figures VII.4-VII.9. Using TCE as an example, the value of $\mathrm{K}_{\mathrm{S}}$ was obtained as a function of mole fraction of TCE in the micelles ( $\mathrm{X}_{\mathrm{TCE}}$ ). At the very low TCE concentrations of interest in the feed groundwater ( $<10 \mathrm{ppm}$ ), the $\mathrm{K}_{\mathrm{s}}$ of interest will be essentially at infinite dilution or near $\mathrm{X}_{\mathrm{TCE}}=0$ on the graphs. The data at higher values of $\mathrm{X}_{\mathrm{TCE}}$ allow accurate extrapolation to the infinite dilution case.

The three surfactant systems for which solubilization data are shown in Figures VII.4-VII.9 are (1) DOWFAX 8390 at $20^{\circ} \mathrm{C}$, (2) the cationic surfactant/DOWFAX $3 \mathrm{~B} 2$ mixture $\left(10 / 90\right.$ mole ratio) at $10^{\circ} \mathrm{C}, 20^{\circ} \mathrm{C}$, and $30^{\circ} \mathrm{C}$, and (3) cetylpyridinium chloride $(\mathrm{CPC})$ at $20^{\circ} \mathrm{C}$ and $30^{\circ} \mathrm{C}$. All surfactant concentrations are constant at $0.05 \mathrm{M}$ - the value of $\mathrm{K}_{\mathrm{S}}$ is 
generally independent of surfactant concentration $(65,66)$. The value of $\mathrm{K}_{\mathrm{S}}$ at $\mathrm{X}_{\mathrm{TCE}}=0$ is similar for all of these surfactant systems. This is consistent with the solubilization locus being in the interior of the micelle or in the palisade layer (66), so the nature of the hydrophilic group does not have a large effect on solubilization. Also, temperature does not have a significant effect on the solubilization constant, a commonly encountered result in these types of systems (으). These results are significant because they indicate that the separation efficiency is not significantly temperature dependent. Since groundwater at varying temperatures could be encountered at different times of year or different locales, this makes the system robust with respect to that important variable.

As an example of the significance of the measured value of $\mathrm{K}_{\mathrm{s}}$ at infinite dilution of about $120 \mathrm{M}^{-1}$, consider a retentate with a concentration of $0.25 \mathrm{M}$ DOWFAX 8390. In such a system, the ratio of solubilized to unsolubilized TCE will be 30 or the ratio of TCE in the permeate to that in the retentate will be $1 / 31$. If the retentate to feed volume ratio is $1 / 5$, the percentage of the feed stream TCE remaining in the retentate stream after one stage would be 88.6 percent. Of course, as will be discussed later in this report, the material balances in a real system with vacuum strippers and recycle streams are more complex, but this simple calculation indicates the type of separation attainable in one stage. As will be seen, we estimate the optimum number of stages to attain a 99 percent removal of TCE from the feed to be about four.

The reluctance to use the cationic/anionic surfactant mixtures because of potential precipitation problems has already been mentioned. 
The leakage of surfactant into the ultimate permeate stream must be minimal or a downstream surfactant recovery step is necessary. The permeate concentration of CPC is about $0.8 \mathrm{mM}$ while that of DOWFAX 8390 is about $0.3 \mathrm{mM}$. Shown in Figure VII.10 is the cost per 1000 gallons of treated groundwater for DOWFAX 8390 lost in the final permeate due to leakage. The cost of surfactant leakage for CPC is of the same magnitude. Both values are too large to avoid downstream treatment, but the molecular weight of the CPC is 340 while that of the DOWFAX 8390 is 642 . As Shown in Figure VII.11 and VII.12 DOWFAX 8390 could be removed from the permeate from the last stage by ultrafiltration with 500 MWCO membranes (the smallest commercially available), although this would be ineffective for CPC. Figure VII.11 shows at the anticipated feed concentrations the relative permeate flux is above 0.90 which indicates minimal negative effects on flux due to concentration polarization. Figure VII.12 shows that 95 percent of the surfactant is rejected (recovered) based on the surfactant feed concentration. The DOWFAX 3B2 for which flux data are shown in Figure VII.3, also has too low a molecular weight for downstream recovery by ultrafiltration. Therefore, considering flux behavior, solubilization constant, and downstream permeate concentrations and recovery potential, DOWFAX 8390 qualifies as the best surfactant investigated so far and all further calculations, designs, and economic estimates in this report will be made assuming use of this surfactant. 


\subsubsection{Spiral Wound Ultrafiltration Unit Results}

Tables VII.2, VII.3, VII.4, VII.5 show data from the spiral wound MEUF unit in which flux and TCE, PCE, DCM rejections were measured at several temperatures $\left(5^{\circ} \mathrm{C}, 15^{\circ} \mathrm{C}\right.$ and $\left.30^{\circ} \mathrm{C}\right)$ and with added salts expected to be present in the ground waters encountered (33 percent ferric sulfate, 33 percent ferrous sulfate, and 34 percent calcium sulfate by weight). The flux values measured are consistent with those shown in Figure VII.3. The temperature does not affect the rejection significantly, as predicted by the equilibrium $\mathrm{K}_{\mathbf{S}}$ data, but increased temperature does cause an increase in flux. The predicted permeate TCE and PCE rejections from the measured $\mathrm{K}_{\mathrm{s}}$ data are also shown in Tables VII.2, VII.3, and VII.4. The average percent rejection of TCE for the single component system was 86 percent and 79 percent for TCE in a mixture of TCE, PCE and DCM. This compares very well with the predicted percent rejection of 85 percent $\left(K_{S}=120 M^{-1}\right)$ based on liquid-vapor equilibrium studies of TCE in micellar solutions. The average percent rejection of PCE was 97 percent for PCE in a mixture of TCE, PCE and DCM. The measured PCE rejection was much better than predicted rejection of 91 percent. This corresponds to a permeate concentration one third the predicted permeate concentration. The data is very consistent for both the $5^{\circ} \mathrm{C}$ and $30^{\circ} \mathrm{C}$ studies which were conducted on separate days with totally different feed solutions. A possible explanation is that the PCE is solubilizing into the cellulose or spacers that comprise the membrane. The 91 percent rejection $\left(K_{S}=210 M^{-1}\right)$ was used for design purposes. The average percent rejection for DCM in a mixture of PCE, TCE 
and DCM was 15 percent. With such a low rejection this is not a good candidate for the MEUF process. The presence of the added salts did not affect the ultrafiltration, as expected, since ultrafiltration is not sensitive to such additives, as would reverse osmosis be, as an example. The flux data in Figure VII.3 and Tables VII.2, VII.3, VII.4, along with the solubilization constant in Figures VII.4, VII.5, VII.6 (confirmed in Table VII.2, VII.3, and VII.4) permit a design of the MEUF stages including the vacuum stripping operation.

\subsubsection{Vacuum Stripping}

A major goal of research performed during this study has been to obtain fundamental data that can be used to determine parameters needed in operating both the MEUF stages and the vacuum stripper used to remove CHC from the concentrated retentate solution. Both the equilibrium solubilization results and initial studies of the removal of $\mathrm{CHC}$ in a vacuum column provide the physical data required to define the operating conditions for the vacuum-stripping and the UF separation processes.

For purposes of estimating the performance of the vacuum stripper, it was assumed that the surfactant concentration in the retentate solution from each of the MEUF stages will be approximately 0.25 M (ca. 160,000 ppm or 1.3 pound per gallon). From Figure VII.3, for DOWFAX 8390, the surfactant upon which the design calculations are based, this is the concentration where the flux becomes unacceptably low, so it represents the MEUF retentate product/stripper feed surfactant concentration. Based on 
an assumed feed groundwater TCE concentration of $1 \mathrm{ppm}$, material balance calculations show that the concentration of TCE in the retentate solutions will vary from about $5 \mathrm{ppm}$ in the first UF stage to approximately $50 \mathrm{ppb}$ in the final stage. However, most of the TCE in the retentate will be bound to the surfactant under these circumstances, and in fact the apparent volatility of TCE from the aqueous solution (determined by the Henry's law constant) will be only approximately 3 percent as great as if the surfactant were not present. This change in apparent volatility or Henry's law constant can be calculated directly from the value of the solubilization equilibrium constant for a given surfactant and the known Henry's law constant for the chlorocarbon in pure water. Thus if the concentration of TCE bound to surfactant micelles is 30 times that of the monomeric TCE, the volatility of TCE from the surfactant solution will be only 1/31 as great as if no surfactant were present.

In designing the vacuum-stripper, the value of the stripping factor (S) will determine how many stages will be required to attain a given percentage removal of TCE, and indirectly this factor will dictate the size and number of transfer units needed. The stripping factor is defined by:

$$
\mathrm{S}=(\mathrm{V} / \mathrm{L}) \mathrm{y} / \mathrm{x}
$$

where $y$ and $x$ are the respective vapor phase and liquid phase mole fractions of TCE and where $V$ and $L$ are the molar flow rates of vapor and liquid through the stripping column. Under the dilute solution conditions prevailing for the TCE, it can be shown that the percentage reduction in 
concentration of TCE from each liquid-phase transfer unit to the next is practically constant when multiple transfer units are used. This implies that the required number of overall transfer units $\left(\mathrm{N}_{\mathrm{tr}}\right)$, the total fractional reduction in TCE concentration (the reduction factor, $R_{F}$ ), and the fractional decrease in TCE concentration from one transfer unit to the next $\left(f_{R}\right)$ are simply interrelated by:

$$
f_{R}=1 / S=R_{F} / N_{t r}
$$

Under the operating conditions of the vacuum-stripper, $\mathbf{S}$ can be chosen to have a value of 4 if the $\mathrm{V} / \mathrm{L}$ ratio is maintained at approximately $1 / 150$, so that each transfer unit will reduce the concentration of TCE by a factor of four (that is, $f_{R}=0.25$ ); and the use of three stripping stages $\left(N_{t r}\right.$ = 3) will lead to an overall reduction in TCE concentration of 1/64 (i.e., $R_{F}=$ .0156). It should be noted that the ratio $\mathrm{V} / \mathrm{L}$ will vary inversely with the value of $S$. For example, if $S=2, V / L=1 / 75$; if $S=4, V / L=1 / 150$; and if $S=$ $6, V / L=1 / 225$ for the conditions assumed in operating the vacuum stripper.

The major factor determining the efficiency of stripping is the value of the dimensionless parameter $m=y / x$, which is determined by the Henry's law constant for TCE dissolved in pure water and the value of $\mathrm{K}_{\mathbf{s}}$. At temperatures in the vicinity of $20^{\circ} \mathrm{C}, \mathrm{y} / \mathrm{x}$ is approximately $1.8 \times 10^{4}$ and the solubilization equilibrium constant is $K_{S}=120 M^{-1}$, so that the $y / x$ value is reduced to approximately 600 at a surfactant concentration of $0.25 \mathrm{M}$. Consequently, $S$ can be held at the reasonable value of 4 by using a vapor to liquid molar ratio of $\mathrm{V} / \mathrm{L}=1 / 150$. The value of $y / x$ does not vary by more than 10 percent in the temperature range $15^{\circ} \mathrm{C}$ to $25^{\circ} \mathrm{C}$. 
Figure VII.13 shows the relation between $R_{F}$ (the factor by which the TCE concentration is reduced in the stripper) and $S$, for an assumed number of transfer units $\left(\mathrm{N}_{\mathrm{tr}}\right)$ equal to two or three in the stripper. From optimization calculations, it is known that a value of $R_{F}$ less than 0.10 will be desirable to prevent an excessive amount of TCE from being returned to the process stream in the $\sim 0.25 \mathrm{M}$ surfactant solution.

Experiments were performed to test the vacuum stripping of $\mathrm{CHC}$ from a concentrated DOWFAX 8390 solution. Initial experiments using a packed column produced significant foaming that filled the tower. Packed glass fiber was unsuccessfully used to break the foam at the top of the column where the vapor exited. The problem was solved by atomization of the feed stream into small droplets providing a large surface area for the volatile organic to volatilize. The results are shown in Tables VII.6 and VII.7 for a single stage vacuum stripper. The sample was sequentially stripped in this unit and an average of 28 percent removal of TCE was observed for the single component system shown in Table VII.6. Shown in Table VII.7 are the results for vacuum stripping of a mixture of PCE, TCE and DCM where the average percent removal was: 17 percent for PCE, 33 percent for TCE and 43 percent for DCM. The results from the stripping experiments show that stripping volatile CHC from surfactant solutions is feasible. 


\subsubsection{Process Design Calculations and Economics}

\subsubsection{Material Balance Calculations}

Material balance calculations were performed for systems of the type shown in Figure VII.1 to investigate the performance of this process, but the number of stages was not necessarily four as shown in Figure VII.1. It is assumed that 99 percent of the TCE in the feed is removed. Since the TCE in this stream is so dilute as to be in the Henry's law region, the actual concentration of the TCE is not important: i.e., removal of 99 percent of a 1 ppm feed requires the same design parameters as removal of 99 percent of a $100 \mathrm{ppb}$ stream. There is one key parameter which indicates the success of the separation: the volume of the retentate streams (sum of retentate streams from each stage) which must be vacuum stripped. If the volume of this combined retentate stream is equal to that of the feed stream, then the feed stream should be directly vacuum stripped and MEUF is not worthwhile. On the other hand, if the size of the retentate streams to be stripped is e.g., $1 / 3$ of the size of the feed stream, this can potentially reduce the load on the stripper sufficiently to more than justify the expense of the MEUF units and result in a more economical overall process.

Tables VII.8, VII.9 and VII.10 shows the results of the material balance calculations. The following variables were investigated: number of stages, surfactant concentration in the retentate, percentage of TCE, PCE

and TCEA in the retentate removed per pass in each stage's vacuum stripper (assumed to be the same for each stage), and solubilization 
constant. In addition to indicating the potential effectiveness of this process, these calculations give an indication of the parameters whose improvement could most enhance the process performance.

The base case involves four stages, a retentate surfactant concentration of 0.25 , a $\mathrm{K}_{\mathrm{g}}$ of $120 \mathrm{M}^{-1}$, and an assumed 90 percent TCE removed from each retentate stream in the vacuum stripper. The retentate streams from the four stages totaled 30.9 percent of the volume of the feed groundwater. If 2 stages are used, the volume percent is 64.6 percent; if 3 stages are used, it is 39.2 percent; if 5 stages are used, it is 27.2 percent. Hence the number of stages is important, but the advantages of adding stages beyond four diminish rapidly. Hence, four stages are chosen as a base case.

If the retentate surfactant concentration is increased to $0.3 \mathrm{M}$ by selection of a superior surfactant or surfactant mixture, this volume percent decreases from 30.9 percent to 26.0 percent. This potential improvement is one of the reasons for the emphasis on surfactant selection investigation in future research.

If the value of $\mathrm{K}_{\mathrm{S}}$ increases from $120^{-1}$ to $150 \mathrm{M}^{-1}$, the volume percent of retentate compared to feed decreases from 30.9 percent to 25 percent, indicating the promise of increasing solubilization capacity for process improvement.

If the percentage of TCE assumed to be removed from each retentate stream in the vacuum stripper is increased from 90 percent to 99 percent, the volume percent of retentate compared to feed decreases from 30.9 
percent to 28.2 percent, indicating the relative modest improvements to be gained by stripping the retentate harder.

Finally, if the retentate surfactant concentration is increased to 0.3 $M$ and the value of $K_{8}$ increases to $150 M^{-1}$ and if the percentage of TCE assumed to be removed from each retentate stream in the vacuum stripper is increased to 99 percent, the volume percent of retentate compared to the feed decreases from 30.9 percent to 19.0 percent, indicating the substantial improvements possible by reasonable possible improvements in the process.

\subsubsection{Economic Evaluation of Process}

The economics presented in this section are based on processing 100,000 gallons per day of TCE contaminated ground water. Capital and operating costs are based on vendor quotes and general cost correlations from standard sources such as Peters and Timmerhaus (68). The equipment cost was divided into five major categories:

1. MEUF Ultrafiltration Units

9 units at $\$ 35,474$

$\$ 319,000$

123 membranes $\$ 1100$

$\$ 135,000$

(The 9 units are skid mounted units requiring only minor modifications)

2. Vacuum Stripper Column

$\$ 65,000$

3. Vacuum Pump

$\$ 28,000$ 
Total Fixed-Capital Investment was calculated by:

Direct Costs

Purchased Equipment

Purchased-Equipment Installation

Instrumentation and Controls (installed)

Piping (installed)

Electrical (installed)

Buildings (including services)

Yard Improvements

Service Facilities (installed)

Indirect Costs

Engineering and Supervision

Construction Expense

Contractors Fees

Contingency

Total Fired Capital Investment
(\%)

59

6

2

3

2

3

2

8

4

4

2

5 Percent

$\$ 1,157,627$ 
Operating Expenses:

Major Energy Costs:

MEUF

9 units 4.2 hp per unit

$28.1 \mathrm{kwh}$

Vacuum Pump

1 unit $10 \mathrm{hp}$ per unit

$7.4 \mathrm{kwh}$

Refrigeration

1 unit $7.3 \times 10^{4} \mathrm{BTU} / \mathrm{h}$

$21.4 \mathrm{kwh}$

Total $57 \mathrm{kwh}$

Assuming $\$ 0.07$ per $k w h$ the annual cost of electricity is: $\$ 34,433$

Other Operating Expenses:

Annual cost of membrane replacement:

$\$ 38,600$

(assumes 3.5 year membrane

life $(\$ 135,000 / 3.5))$

Annual cost of lost surfactant:

$\$ 2,000$

(assumes permeate concentration of

5 micromoles per liter)

Depreciation of Capital Investment:

$\$ 57,880$

(based on 20 year plant life)

Annual Personnel costs:

$\$ 50,000$

Miscellaneous:

$\$ 5.000$

(valves, pressure gauges, etc)

Total Annual Operating Expenses

$\$ 187,913$ 
The above values are for a plant processing $36,500,000$ gallons annually at a cost of $\$ 5.14$ per 1000 gallons of processed groundwater (in 1992 dollars). At the flow rate of 100,000 gallon/day, the economies of scale may not be fully realized. Using air stripping with appropriate pollution control devices results in approximately $\$ 4$ to $\$ 6 / 1000$ gallons (67). The capitalized cost is $\$ 1,291,514$ based on a 20 year plant life and an interest rate of 12 percent. The replacement of membranes may also be viewed as a capitalized cost of $\$ 412,300$ based on a 3.5 year useful life and an interest rate of 12 percent. Including both the original fixed capital investment and the cost of replacement membranes, the capitalized cost of the process is $\$ 1,703,814$. The annual cost of capital for the first year is $\$ 138,915$ based on the total fixed captial investment at an annual interest rate of 12 percent. By including the annual cost of capital as an expense, the cost per 1000 gallons of processed groundwater is increased by $\$ 3.86$ to $\$ 9.00$ for the first year of operation. This preliminary economic estimate for this process based on only a feasibility study shows that it can result in approximately the same cost for treatment of groundwater as established techniques.

Four different membranes are used in the TCE system:
a. 50,000 MWCO membrane (stage \#1)
b. 50,000 MWCO high flux membrane (stages 2 \& 3)
c. 10,000 MWCO membrane (stage \#4)
d. $500 \mathrm{MWCO}$ membrane (surfactant recovery) 
The first three stages use 50,000 MWCO membranes but the high flux $50,000 \mathrm{MWCO}$ membranes used in the second and third stages have twice the flux compared to the $50,000 \mathrm{MWCO}$ membranes used in the first stage. The high flux membranes are very susceptible to fouling by particles and are therefore used after the first. stage standard 50,000 MWCO membranes, which are not as susceptible to fouling and provide permeate, free of harmful fouling particulates, to the follow-on stages. Stage four uses 10,000 MWCO membranes which are capable of significantly reducing the surfactant concentration in the permeate stream when compared to a 50,000 MWCO membrane. However, the flux for a 10,000 MWCO membrane is one half that of a $50,000 \mathrm{MWCO}$ membrane in the first stage. There is one more stage of membranes not associated with the MEUF process but used for the recovery of surfactant from the stage four permeate stream. This surfactant recovery step uses $500 \mathrm{MWCO}$ membranes with a relative flux approaching that of the 10,000 MWCO membrane mainly because the 500 MWCO membranes are operated within a very low surfactant retentate concentration range where concentration polarization effects on flux are small. The majority of the membranes, almost 70 percent, are located in stage four and the surfactant recovery stage. A total of only 16 percent of the membranes are located in stages 2 and 3. If one were to consider a similar system for the recovery of PCE from groundwater, the system would require one less stage (either stage 2 or 3 ) compared to a system designed for TCE. This is due to a $K_{S}=210 M^{-1}$ for PCE, compared to a $K_{S}=120 \mathrm{M}^{-1}$ for TCE. However, this does not translate into a 25 percent reduction in capital costs and operating costs when 
comparing a PCE recovery system of three stages to a TCE recovery system of four stages. A reduction of only around 8 percent of the capital cost and operating costs of the MEUF portion of the system would be realized by reducing the MEUF portion of the system by one MEUF stage for PCE recovery.

The requirement for one less MEUF stage for PCE recovery when compared to TCE recovery also reduces the size of the total retentate stream requiring regeneration by the stripping sections. The reduction in the size of the retentate stream requiring regeneration is significant enough to totally eliminate one of the stripper sections. The entire capital cost and operating cost of one stripper section (condenser, vacuum pump, refrigeration, etc) would be saved with the reduction of the number of strippers from 4 to 3 . The strippers were sized with two transfer units which were more than adequate for TCE and a reevaluation of the towers for PCE shows that two transfer units are more than sufficient for this application.

This is the first major attempt to evaluate a very young technology for application to concentrate a specific class of compounds on a commercial/industrial scale. The process has tremendous potential if developments can be made in the foliowing areas:

a. The operating range of the surfactant retentate concentration needs to be significantly broadened to reduce the size of the retentate stream requiring regeneration.

b. A significant increase of solubility of the target organic in the micelle, an increase in the distribution constant $\left(\mathrm{K}_{\mathrm{g}}\right)$, would reduce the number of stages. 
c. Perhaps the most significant development is to reduce the permeate surfactant concentration or improve the recovery of the surfactant in the final permeate stream on a more cost effective basis.

If significant progress were accomplished in just the last category, the number of membranes used in this process could be reduced by 50 percent. Moderate progress in the first two categories could further reduce the total number of membranes required to 25 percent of the values used for the economics in this study. The MEUF process has substantial potential for improvement and optimization (reasonably, a factor of 2 to 4 improvement in the cost/unit volume might be expected). Therefore, MEUF shows considerable promise as an improved technology for this application.

\subsection{Conclusions}

Of the four surfactants evaluated, DOWFAX 8390 has the best overall performance when considering CHC solubility, permeate flux, and surfactant recovery. Separation of CHC from water using the MEUF process with DOWFAX 8390 has rejections very similar to predicted rejections assuming that the $\mathrm{CHC}$ is in equilibrium accross the membrane; TCE rejections of 70 to 88 percent, and PCE rejections of 96 to 98 percent were observed for a single pass operation. Decreases in feed stream temperature reduces permeate flux.

The CHC componets studied were found to be easily volatilized from a surfactant solution. The MEUF process in conjunction with vacuum stripping can remove $99+$ percent of the $\mathrm{CHC}$ from groundwater at $\$ 5.23$ per 1000 gallons 
compared to $\$ 4$ to $\$ 6 / 1000$ gallons for fully developed technologies for a 100,000 gallon/day facility.

The MEUF process is an extremely young concept and has substantial potential for improvement and optimization. Studies need to continue with developments to: (1) extend the operating range of surfactant concentrations on the retentate side of the membrane, (2) enhance CHC solubility in the micelle, (3) improve permeate flux, and (4) decrease permeate surfactant concentrations. Reduction in the cost/unit of one-half to one-fourth is anticipated. This study of MEUF shows enough promise to justify further work to improve its performance. 
Table VII.2: MEUF DATA FOR TCE USING DOWFAX 8390 AT VARIOUS TEMPERATURES WITH ADDED SALTS

\begin{tabular}{|c|c|c|c|c|c|c|}
\hline $\begin{array}{c}\text { TEMPERATURE } \\
\left({ }^{\circ} \mathrm{C}\right)\end{array}$ & $\begin{array}{c}\text { [ADDED SALT } \\
(\mathrm{ppm})\end{array}$ & $\begin{array}{c}\text { FLUX } \\
\mathrm{g} /\left(\mathrm{min} \mathrm{cm}^{2}\right)\end{array}$ & $\begin{array}{c}\text { AVERAGE } \\
\text { RETENTATE } \\
\text { [TCE] } \\
(\mathrm{ppb})\end{array}$ & $\begin{array}{c}\text { PERMEATE } \\
\text { [TCE] } \\
(\mathrm{ppb})\end{array}$ & $\begin{array}{c}\text { REJECTION } \\
(\%)\end{array}$ & $\begin{array}{c}\text { PREDICTED } \\
\text { REJECTION } \\
(\%)\end{array}$ \\
\hline 30 & 0 & .092 & 860 & 99 & 88 & 85 \\
\hline \multirow{6}{*}{30} & 100 & .097 & 645 & 91 & 86 & 85 \\
\hline 15 & 0 & .069 & 835 & 126 & 85 & 85 \\
\hline 15 & 100 & .068 & 745 & 104 & 86 & 85 \\
\hline
\end{tabular}

ADDED SAlt IS A MIXTURE OF 33 PERCENT CAlCIUM SUlfate, 33 PERCENT FERRIC SULFATE, AND 33 PERCENT FERROUS SULFATE BY WEIGHT. 
Table VI.3: MEUF DATA FOR PCE IN A MIXTURE WITH TCE AND DCM USING DOWFAX 8390 AT VARIOUS TEMPERATURES

\begin{tabular}{|c|c|c|c|c|c|c|}
\hline $\begin{array}{l}\text { SAMPLE } \\
\text { NUMBER }\end{array}$ & $\begin{array}{l}\text { TEMPERATURE } \\
\text { ('C) }\end{array}$ & $\underset{g /\left(\min c^{2}\right)}{\operatorname{FLUX}}$ & $\begin{array}{c}\text { AVERAGE } \\
\text { RETENTATE } \\
\text { [PCE] } \\
\text { (ppm) }\end{array}$ & $\begin{array}{c}\text { PERMEATE } \\
\text { [PCE] } \\
\text { (ppb) }\end{array}$ & $\begin{array}{l}\text { REJECTION } \\
\text { (\$) }\end{array}$ & $\begin{array}{c}\text { PREDICTED } \\
\text { REJECTION } \\
\text { (\%) }\end{array}$ \\
\hline 1 & 5 & .054 & 1.6 & 50 & 97 & 91 \\
\hline 2 & 5 & .054 & 2.7 & 100 & 96 & 91 \\
\hline 3 & 5 & .054 & 6.2 & 210 & 97 & 91 \\
\hline 4 & 5 & .054 & 7.0 & 270 & 96 & 91 \\
\hline 5 & 5 & .054 & 10.3 & 340 & 97 & 91 \\
\hline 6 & 5 & .054 & 13.9 & 360 & 97 & 91 \\
\hline 7 & 5 & .054 & 16.9 & 340 & 98 & 91 \\
\hline 8 & 30 & .093 & 2.2 & 140 & 94 & 91 \\
\hline 9 & 30 & .093 & 3.6 & 157 & 96 & 91 \\
\hline 10 & 30 & .093 & 6.4 & 183 & 97 & 91 \\
\hline 11 & 30 & .093 & 7.8 & 262 & 97 & 91 \\
\hline 12 & 30 & .093 & 10.6 & 217 & 98 & 91 \\
\hline 13 & 30 & .093 & 13.2 & 340 & 97 & 91 \\
\hline
\end{tabular}


Table VII.4: MEUF DATA FOR TCE IN A MIXTURE WITH PCE AND DCM USING DOWFAX 8390 AT VARIOUS TEMPERATURES

\begin{tabular}{|c|c|c|c|c|c|c|}
\hline $\begin{array}{l}\text { SAMPLE } \\
\text { NUMBER }\end{array}$ & $\begin{array}{l}\text { TEMPERATURE } \\
\text { ('C) }\end{array}$ & $\underset{g /\left(\min \mathrm{cm}^{2}\right)}{\text { FuX }}$ & $\begin{array}{c}\text { AVERAGE } \\
\text { RETENTATE } \\
\text { [TCE] } \\
\text { (ppm) }\end{array}$ & $\begin{array}{l}\text { PERMEATE } \\
\text { [TCE] } \\
\text { (ppm) }\end{array}$ & $\begin{array}{l}\text { REJECTION } \\
\text { (\$) }\end{array}$ & $\begin{array}{c}\text { PREDICTED } \\
\text { REJECTION } \\
\text { (\%) }\end{array}$ \\
\hline 1 & 5 & .054 & 1.4 & .24 & 83 & 85 \\
\hline 2 & 5 & .054 & 2.3 & .41 & 82 & 85 \\
\hline 3 & 5 & .054 & 4.2 & 1.09 & 74 & 85 \\
\hline 4 & 5 & .054 & 5.6 & 1.65 & 71 & 85 \\
\hline 5 & 5 & .954 & 9.2 & 2.72 & 70 & 85 \\
\hline 6 & 5 & .054 & 10.8 & 2.27 & 79 & 85 \\
\hline 7 & 5 & .054 & 13.4 & 2.49 & 81 & 85 \\
\hline 8 & 30 & .093 & 1.6 & .32 & 80 & 85 \\
\hline 9 & 30 & .093 & 3.4 & .50 & 85 & 85 \\
\hline 10 & 30 & .093 & 4.9 & 81 & 84 & 85 \\
\hline 11 & 30 & .093 & 7.9 & 1.7 & 79 & 85 \\
\hline 12 & 30 & .093 & 10.5 & 1.9 & 82 & 85 \\
\hline 13 & 30 & .093 & 12.5 & 2.5 & 80 & 85 \\
\hline
\end{tabular}


Table VII.5: MEUF DATA FOR DCM IN A MIXTURE WITH PCE AND TCE USTN DOWFAX 8390 AT VARIOUS TEMPERATURES

\begin{tabular}{|c|c|c|c|c|c|}
\hline $\begin{array}{l}\text { SAMPLE } \\
\text { NUMBER }\end{array}$ & $\begin{array}{l}\text { TEMPERATURE } \\
\text { ('C) }\end{array}$ & $\frac{\text { FLUX }}{2 /\left(\min \mathrm{cm}^{2}\right)}$ & $\begin{array}{c}\text { AVERAGE } \\
\text { RETENTATE } \\
\text { [DCM] } \\
\text { (pon) }\end{array}$ & $\begin{array}{c}\text { PERMPATE } \\
\text { (DCM] } \\
\text { (ppm) }\end{array}$ & $\begin{array}{l}\text { REJECTION } \\
\text { (\$) }\end{array}$ \\
\hline 1 & 5 & .054 & 1.3 & 9 & 31 \\
\hline 2 & 5 & .054 & 1.5 & 2.0 & - \\
\hline 3 & 5 & .054 & 4.6 & 4.1 & 11 \\
\hline 4 & 5 & .054 & 5.5 & 6.3 & - \\
\hline 5 & 5 & .054 & 8.4 & 6.5 & 23 \\
\hline 6 & 5 & .054 & 9.6 & 78 & 19 \\
\hline 7 & 5 & .054 & 12.3 & 8.6 & 30 \\
\hline 8 & 30 & .093 & 1.1 & 1.1 & - \\
\hline 9 & 30 & .093 & 2.7 & 28 & - \\
\hline 10 & 30 & .093 & 4.5 & 3.5 & 22 \\
\hline 11 & 30 & .093 & 6.4 & 7.0 & - \\
\hline 12 & 30 & .093 & 9.8 & 6.7 & 25 \\
\hline 13 & 30 & .093 & 112 & 7.2 & 36 \\
\hline
\end{tabular}


Table VII.6: RESULTS OF VACUUM STRIPPING OF TCE FROM A DOWFAX 8390 SURFACTANT SOLUTION

\begin{tabular}{|c|c|c|}
\hline \# OF TIMES STRIPPED & $\begin{array}{c}\text { CONCENTRATION } \\
\text { TCE (PPM) }\end{array}$ & $\begin{array}{c}\text { TCE REMOVED } \\
(\%)\end{array}$ \\
\hline FEED & 13.9 & \\
\hline 1 & 8.4 & 40 \\
\hline 2 & 6.5 & 22.6 \\
\hline 3 & 5.7 & 12.3 \\
\hline 4 & 4.3 & 24 \\
\hline 5 & 3.1 & 28 \\
\hline 6 & 2.2 & 29 \\
\hline 7 & 1.3 & 40 \\
\hline 8 & 0.93 & 28 \\
\hline
\end{tabular}


Table VII.7: RESULTS OF VACUUM STRIPPING OF MIXTURES OF PCE, TCE AND DCE FROM A $0.25 \mathrm{M}$ SOLUTION OF DOWFAX 8390

\begin{tabular}{|c|c|c|c|c|c|c|}
\hline $\begin{array}{c}\text { OF } \\
\text { TIMIES } \\
\text { STRIPPED }\end{array}$ & $\begin{array}{c}\text { CONCENTRATION } \\
\text { [PCE] } \\
\text { (PPM) }\end{array}$ & $\begin{array}{c}\text { PCE } \\
\text { REMOVED } \\
(\mathbf{(}) \\
\end{array}$ & $\begin{array}{l}\text { CONCENTRATION } \\
\text { [TCE] } \\
\text { (PPM) } \\
\end{array}$ & $\begin{array}{c}\text { TCE } \\
\text { REMOVED } \\
\text { (\$) } \\
\end{array}$ & $\begin{array}{l}\text { CONCENTRATION } \\
\text { [DCM] } \\
\text { (PPM) }\end{array}$ & $\begin{array}{c}\text { DCM } \\
\text { REMOVED } \\
\text { (\$) } \\
\end{array}$ \\
\hline TEED & 14.77 & & 13.40 & & 12.44 & \\
\hline 1 & 12.51 & 15.3 & 10.36 & 22.7 & $\mathbf{8 . 0 3}$ & 35.5 \\
\hline 2 & 10.82 & 13.5 & 6.64 & 35.9 & 3.59 & 50.4 \\
\hline 3 & 9.23 & 14.8 & 4.55 & 25.5 & 2.2 & 36.9 \\
\hline 4 & 7.25 & 21.4 & 3.35 & 32.4 & .85 & 50 \\
\hline 5 & 5.84 & 19.5 & 2.25 & 32.9 & $>1$ & - \\
\hline 6 & 5.19 & 11.3 & 1.19 & 47 & $>1$ & - \\
\hline 7 & 3.78 & 27.1 & $>1$ & - & $>1$ & - \\
\hline 8 & 3.31 & 12.4 & $>1$ & - & $>1$ & - \\
\hline
\end{tabular}


Table VII.8: RESULTS OF MATERIAL BALANCE CALCULATIONS ON THE MEUF PROCESS FOR TCE

\begin{tabular}{|c|c|c|c|c|c|c|c|c|}
\hline Number of Stages & 4 & 2 & 3 & 5 & 4 & 4 & 4 & 4 \\
\hline $\begin{array}{c}\text { Retentate Surfactant } \\
\text { Concentration } \\
(\mathrm{M})\end{array}$ & 0.25 & 0.25 & 0.25 & 0.25 & 0.30 & 0.25 & 0.25 & 0.30 \\
\hline $\mathrm{K}_{\mathrm{S}}(\mathrm{M}-1)$ & 120 & 120 & 120 & 120 & 120 & 150 & 120 & 150 \\
\hline $\begin{array}{c}\text { TCE Removed from } \\
\text { Retentate in Each } \\
\text { Stripping Column } \\
\text { (\%) }\end{array}$ & 90 & 90 & 90 & 90 & 90 & 90 & 99 & 99 \\
\hline $\begin{array}{c}\text { Sum of All Retentate } \\
\text { Streams/Feed Streams } \\
\text { (Volume/Volume) }\end{array}$ & 0.309 & 0.646 & 0.392 & 0.272 & 0.260 & 0.250 & 0.282 & 0.190 \\
\hline
\end{tabular}


Table VH.9: RESULTS OF MATERIAL BALANCE CALCULATIONS ON THE MEUF PROCESS FOR 1,1,1, TRICHLOROETHANE

\begin{tabular}{|c|c|c|c|c|}
\hline Number of Stages & 2 & 3 & 4 & 5 \\
\hline $\begin{array}{l}\text { Retentate Surfactant } \\
\text { Concentration } \\
\text { (M) }\end{array}$ & 0.25 & 0.25 & 0.25 & 0.25 \\
\hline$K_{S}\left(M^{-1}\right)$ & 180 & 180 & 180 & 180 \\
\hline $\begin{array}{l}\text { 1.1.1. Trichloroethane } \\
\text { Removed from Retentate } \\
\text { in Each } \\
\text { Stripping Column } \\
\text { (\%) }\end{array}$ & 90 & 90 & 90 & 90 \\
\hline $\begin{array}{c}\text { Sum of All Retentate } \\
\text { Streams/Feed Streams } \\
\text { (Volume/Volume) }\end{array}$ & 0.435 & 0.264 & 0.197 & 0.183 \\
\hline
\end{tabular}


Table VII.10: RESULTS OF MATERIAL BALANCE CALCULATIONS ON THE MEUF PROCESS FOR PCE

\begin{tabular}{|c|c|c|c|c|}
\hline Number of Stages & 2 & 3 & 4 & 5 \\
\hline $\begin{array}{c}\text { Retentate Surfactant } \\
\text { Concentration } \\
\text { (M) }\end{array}$ & 0.25 & 0.25 & 0.25 & 0.25 \\
\hline $\mathrm{K}_{\mathrm{S} \text { (M-1) }}$ & 210 & 210 & 210 & 210 \\
\hline $\begin{array}{c}\text { PCE Removed from } \\
\text { Retentate in Each } \\
\text { Stripping Column } \\
\text { (\%) }\end{array}$ & 90 & 90 & 90 & 90 \\
\hline $\begin{array}{c}\text { Sum of All Retentate } \\
\text { Streams/Feed Streams } \\
\text { (Volume/Volume) }\end{array}$ & 0.374 & 0.227 & 0.180 & 0.157 \\
\hline
\end{tabular}




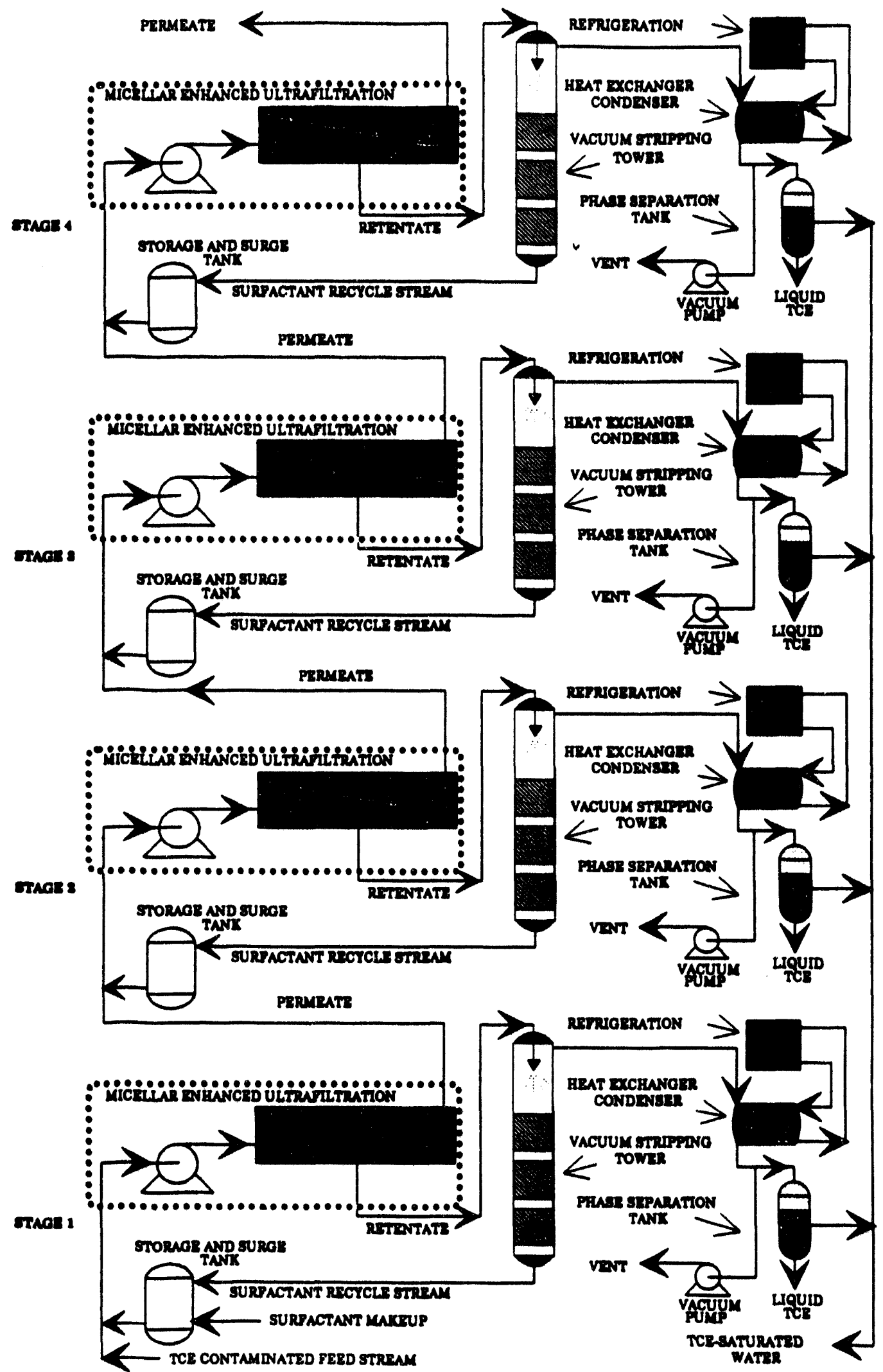

Figure VII.1: Process Flow Diagram for Recovery of TCE from Groundwater Using MEUF in a Four Stage Process 


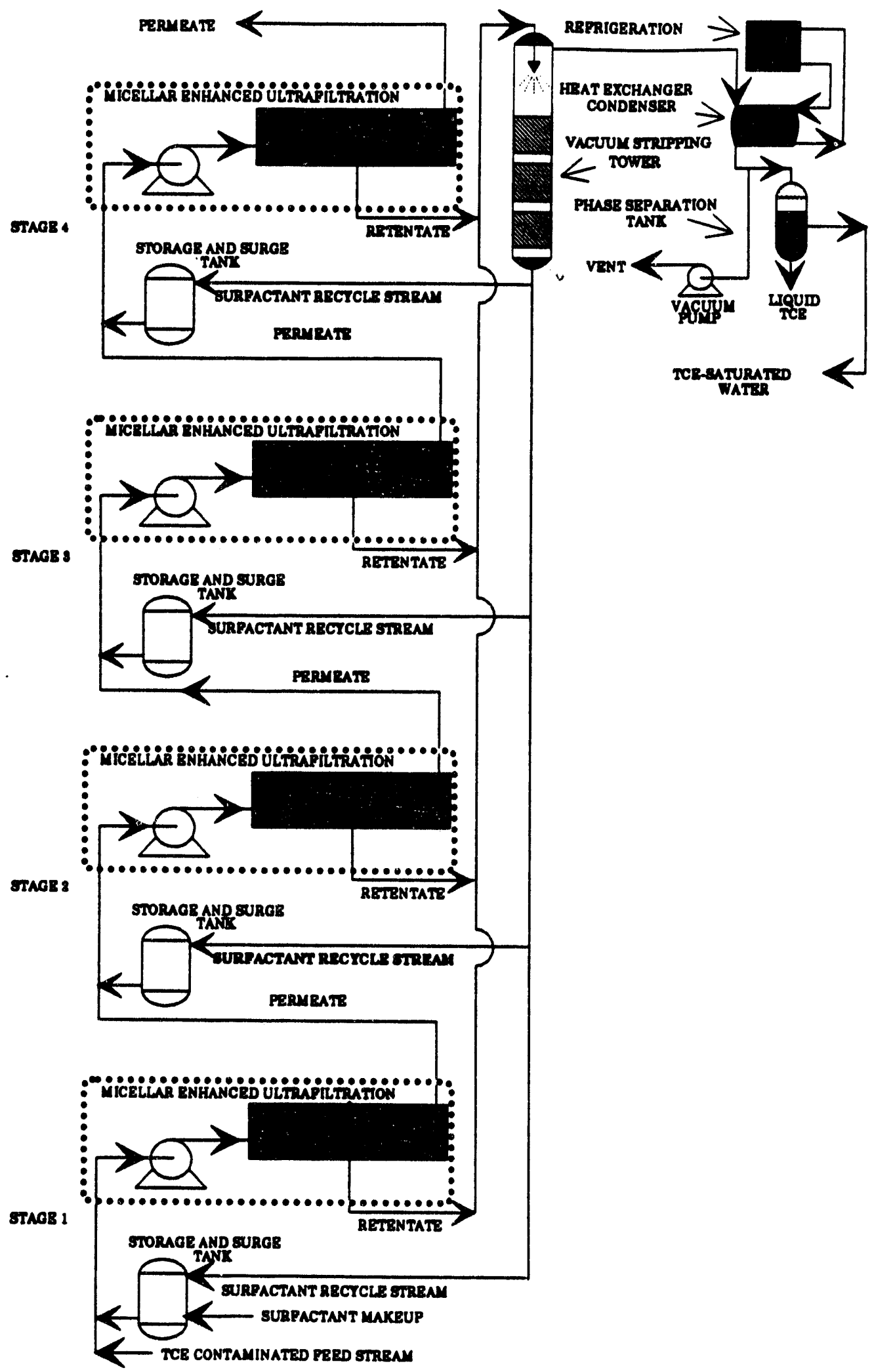

Figure VII.2: Process Flow Diagram for Recovery of TCE from Groundwater Using MEUF in a Four Stage Process and a Single Stripper 


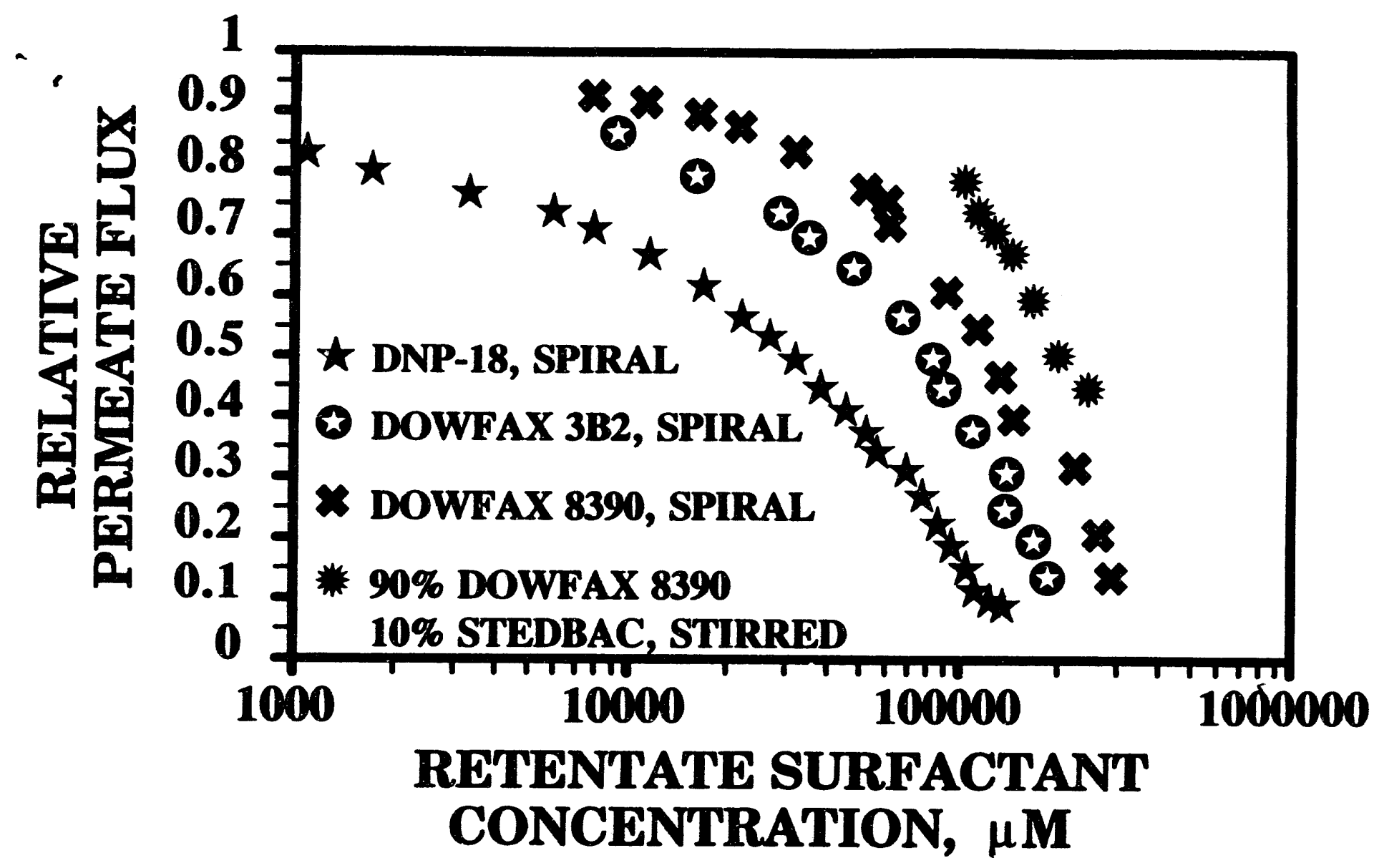

Figure VII.3: The Relative Permeate Flux for Several Surfactants for Spiral Wound and Stirred Cell Studies using 10K MWCO Ultrafiltration Membranes 


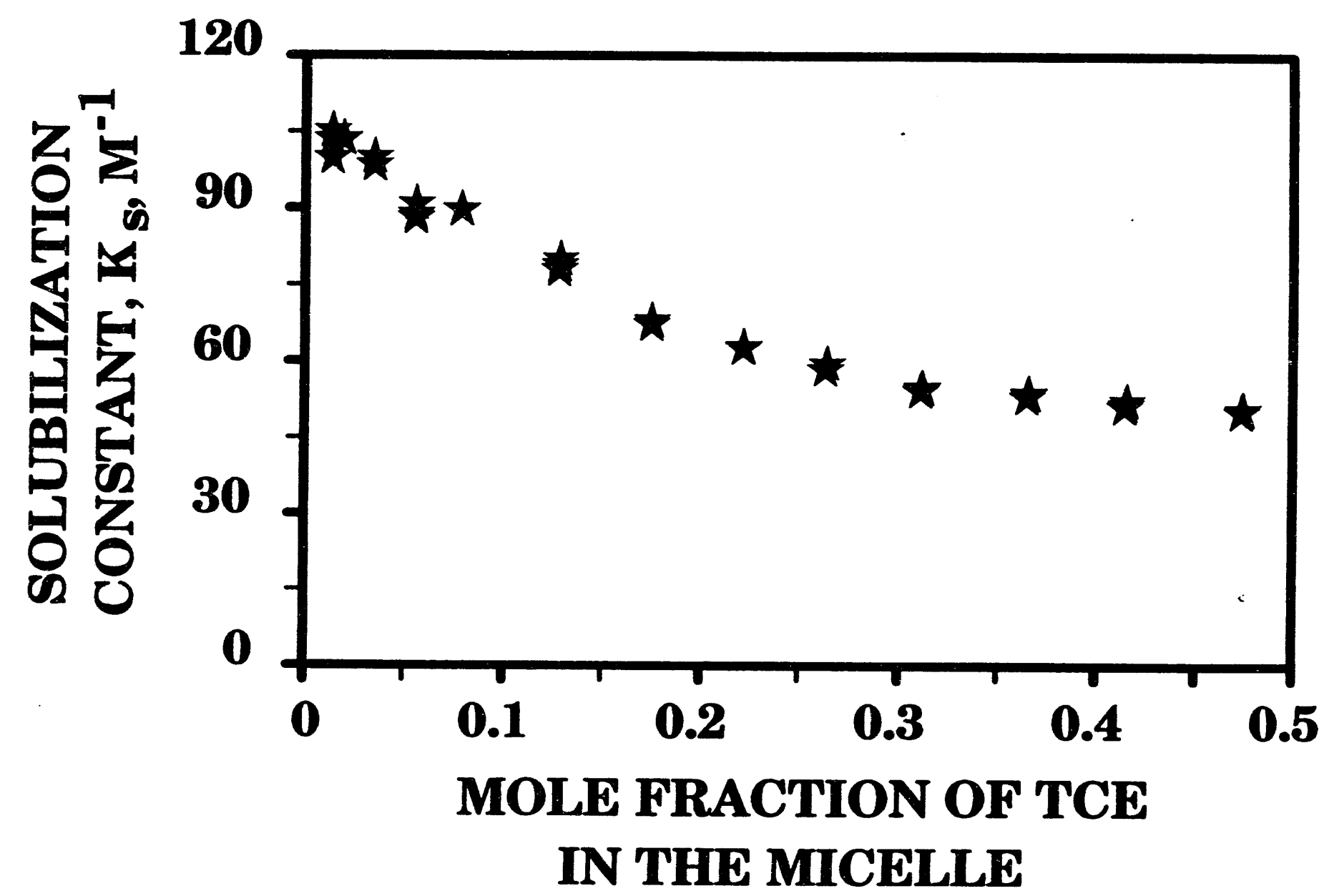

Figure VII.4: The Solubilization Constant of TCE in 0.05 M DOWFAX 8390 


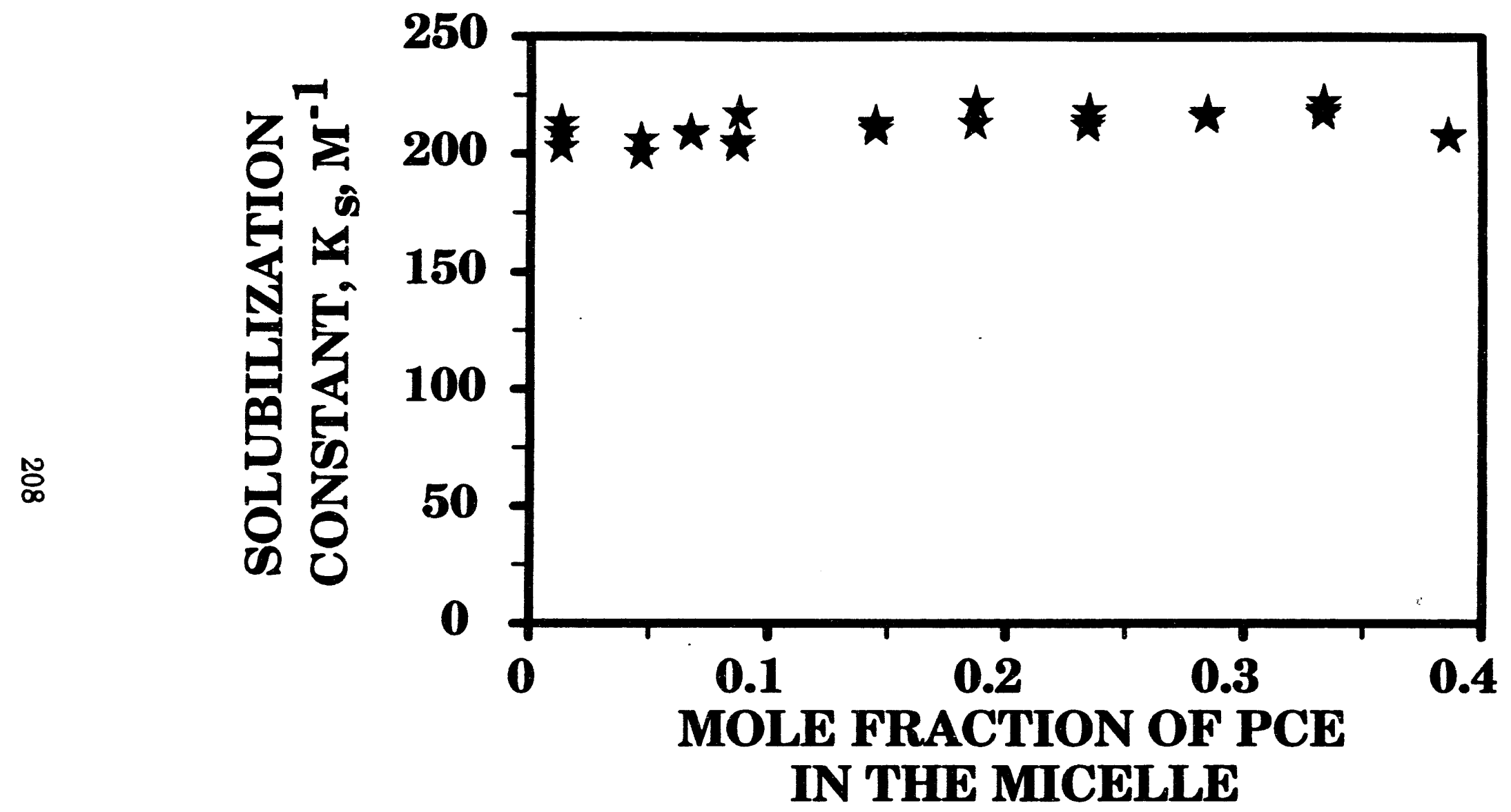

Figure VII.5: The Solubilization Constant of PCE in $0.05 \mathrm{M}$ DOWFAX 8390 at $20^{\circ} \mathrm{C}$ 


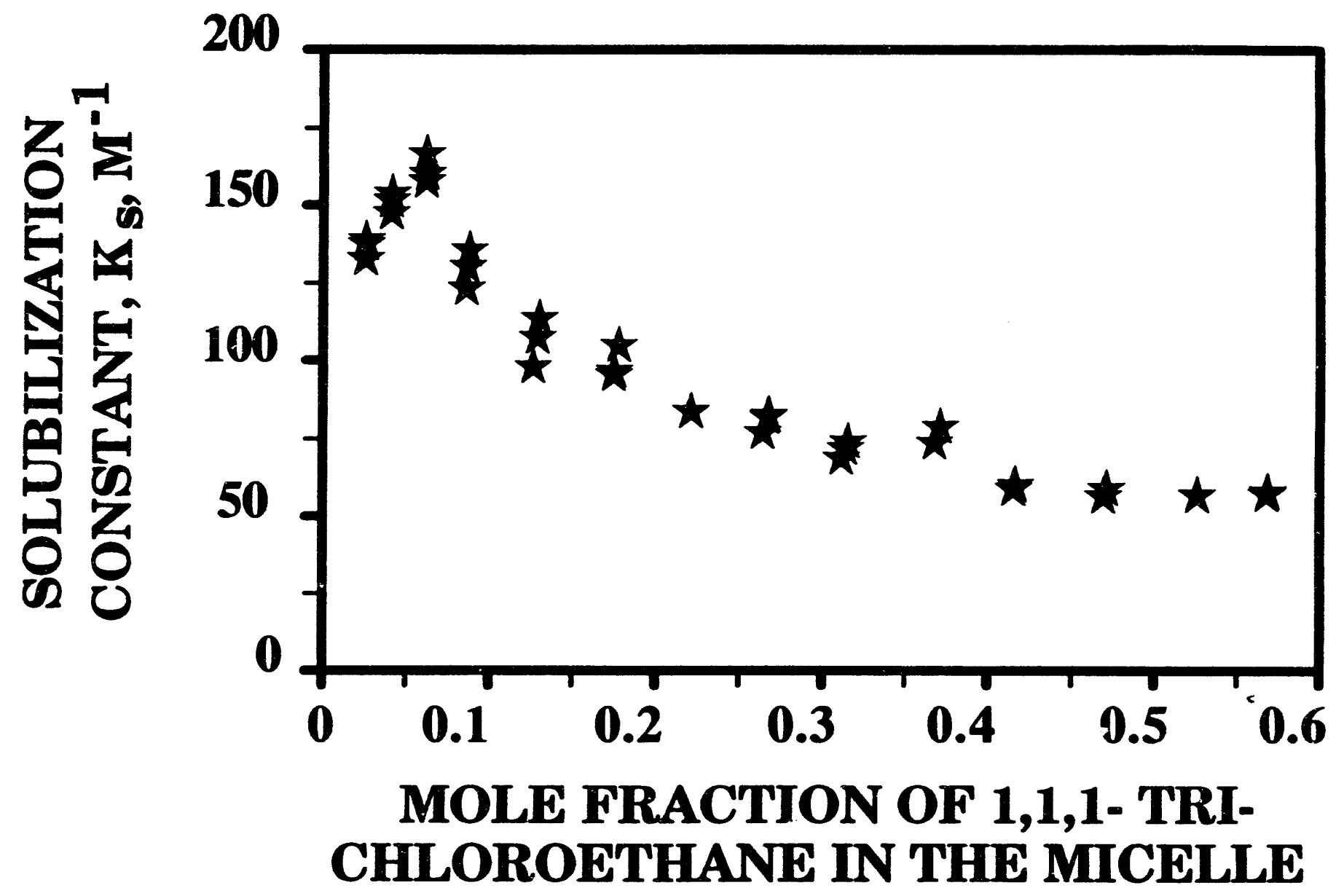

Figure VII.6: The Solubilization Constant of 1,1,1-Trichloroethane in $0.05 \mathrm{M}$ DOWFAX 8390 at $20^{\circ} \mathrm{C}$ 


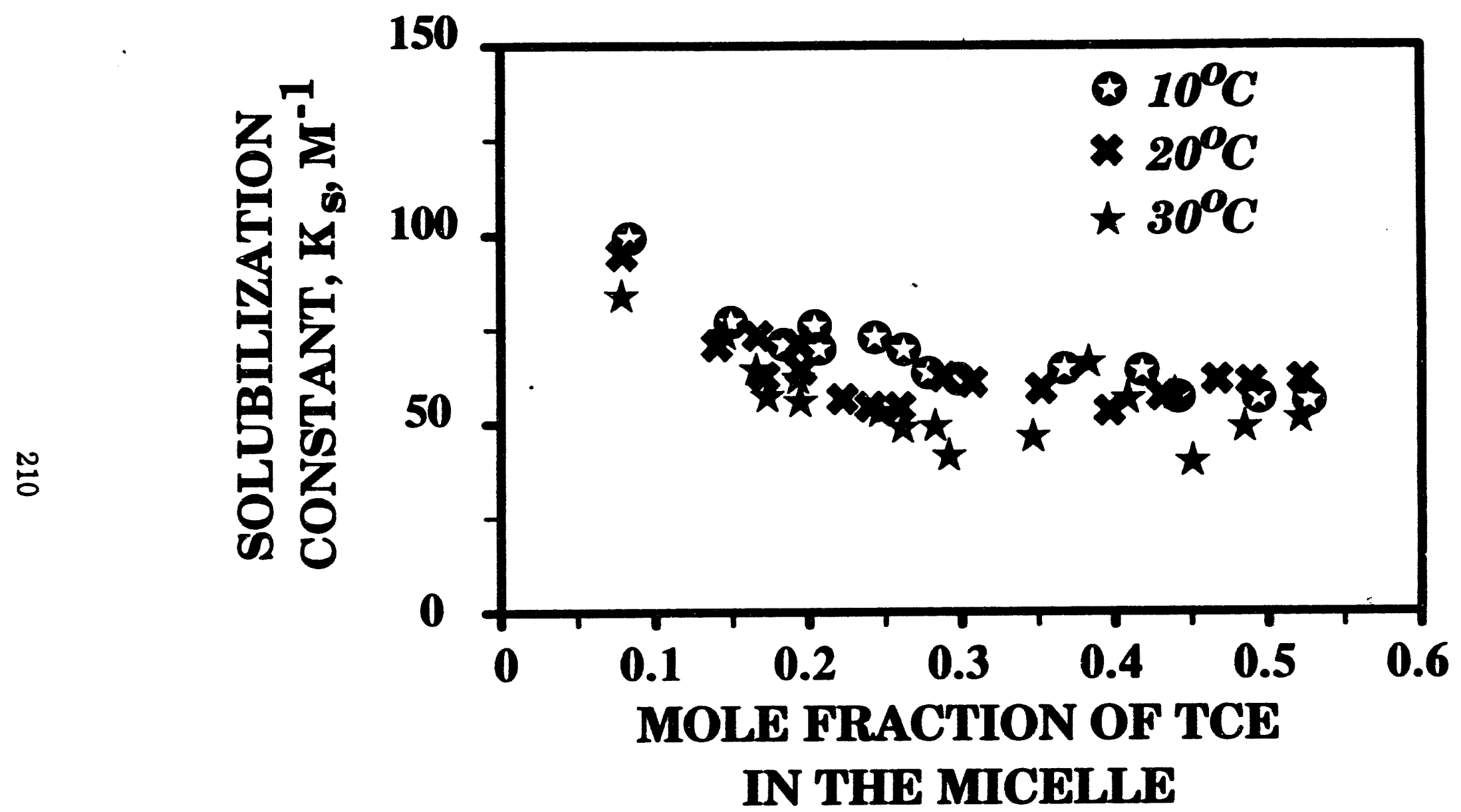

Figure VII.7: The Solubilization Constant of TCE in a 0.05 M Surfactant Solution Composed of 0.9 Mole Fraction DOWFAX 3B2 and 0.1 Mole Fraction STEDBAC 


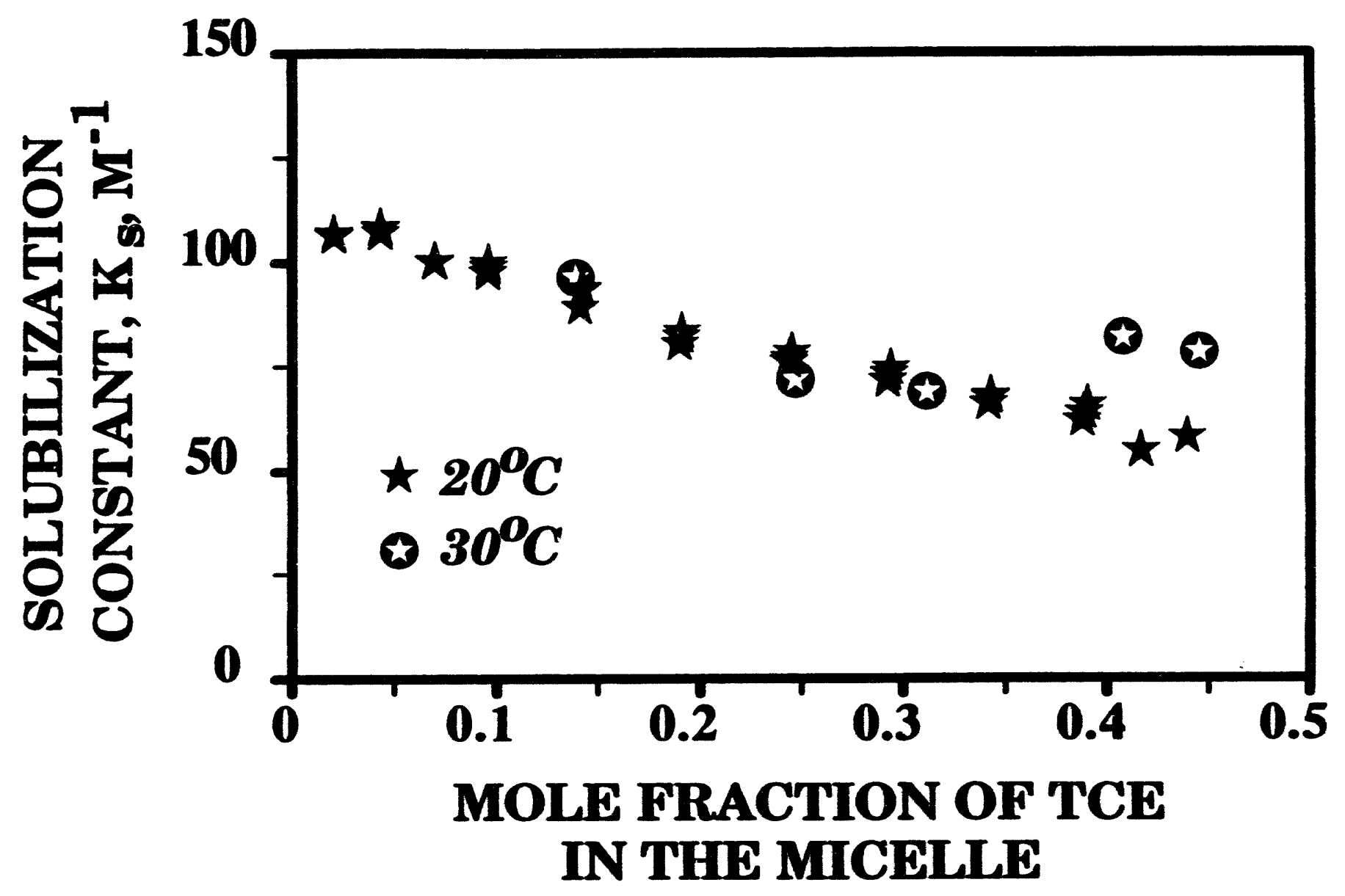

Figure VII.8: The Solubilization of TCE in a 0.05 M CPC Surfactant Solution at $20^{\circ} \mathrm{C}$ and $30^{\circ} \mathrm{C}$ 


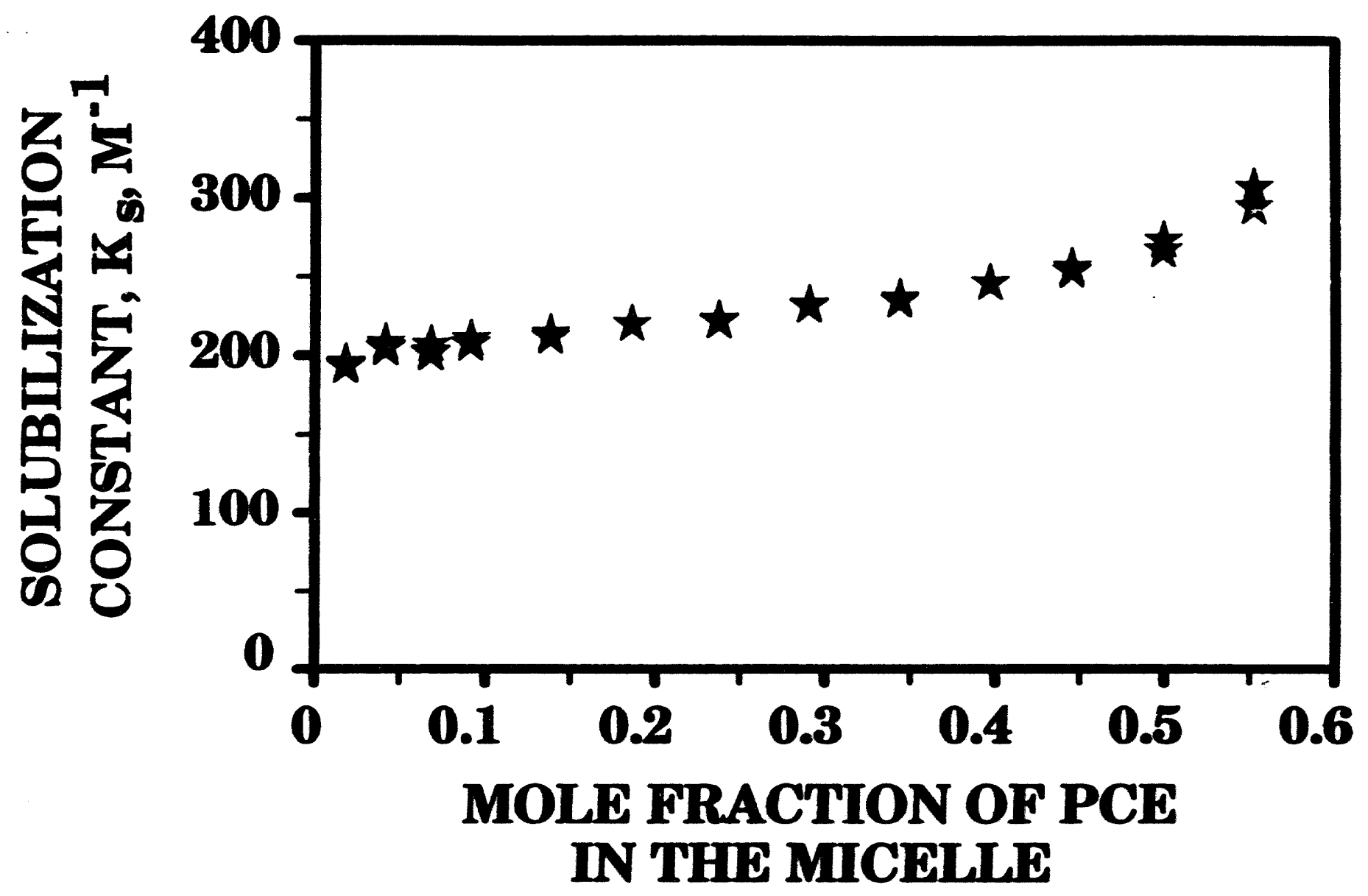

Figure VII.9: The Solubilization Constant of PCE in a 0.05 M CPC Surfactant Solution at $20^{\circ} \mathrm{C}$ 


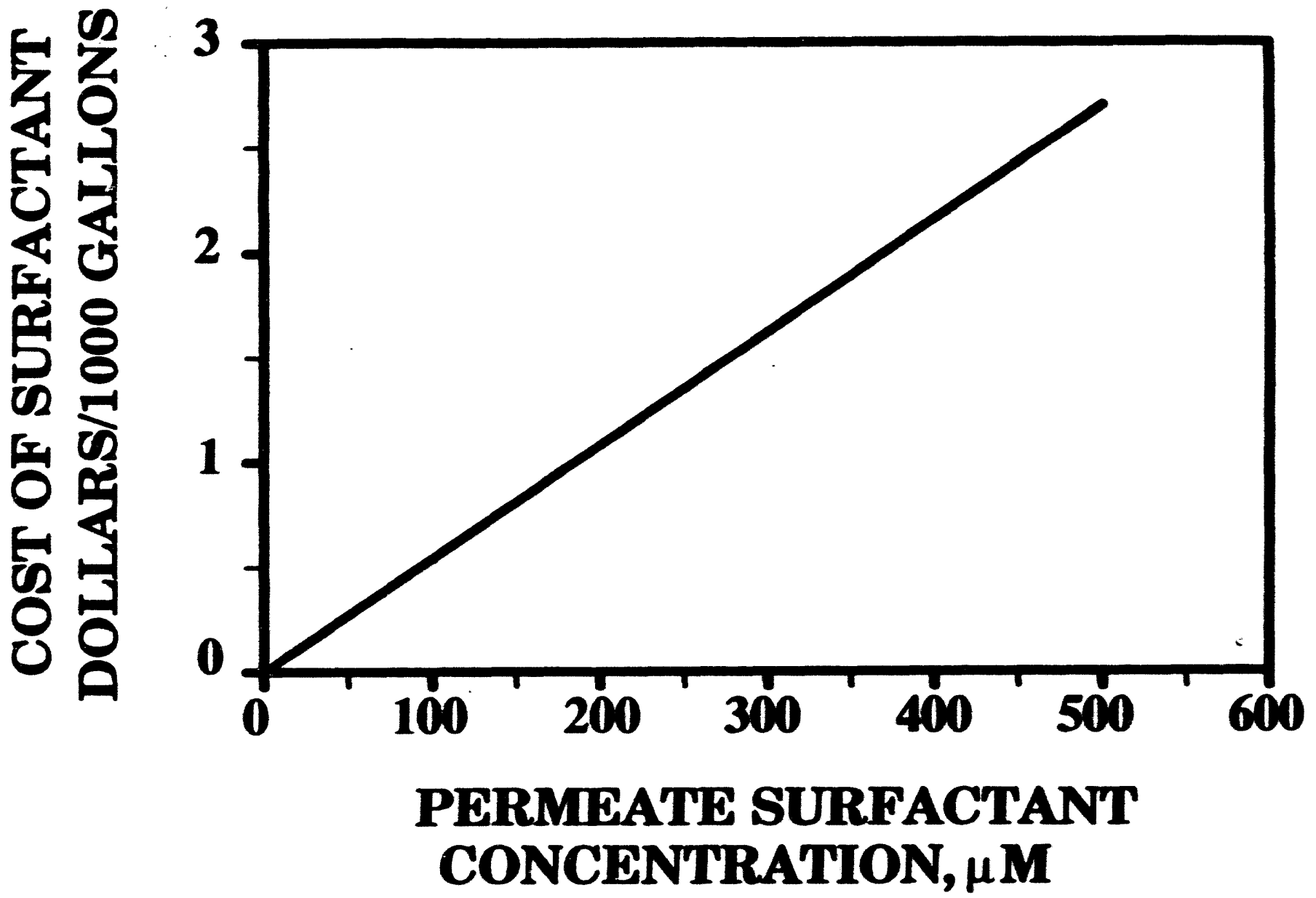

Figure VII.10: The Cost of DOWFAX 8390 Lost in the Final Permeate Stream 


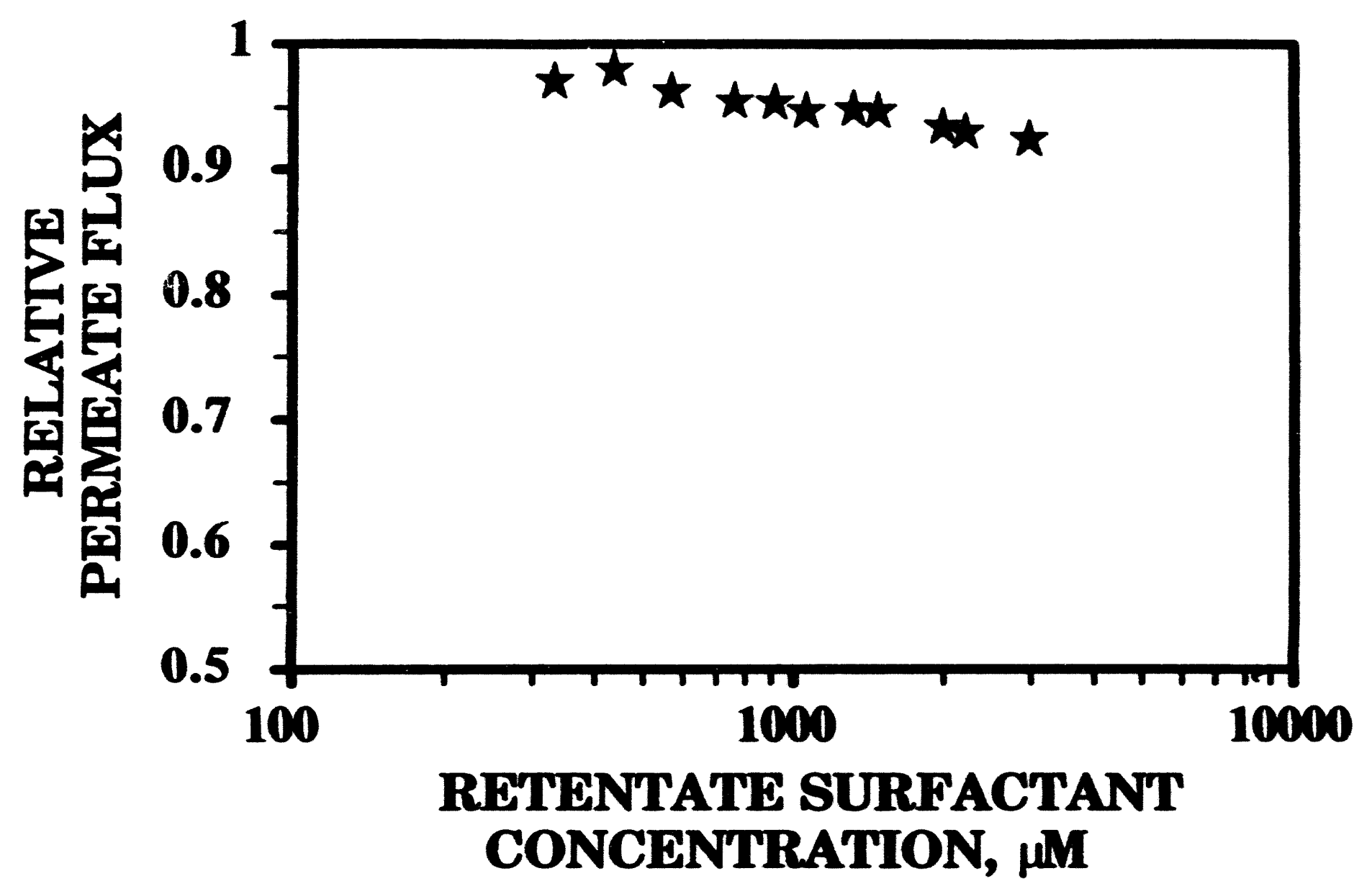

Figure VII.11: The Permeate Flux for DOWFAX 8390 Using a 500 MWCO Spiral Wound Ultrafiltration Membrane 


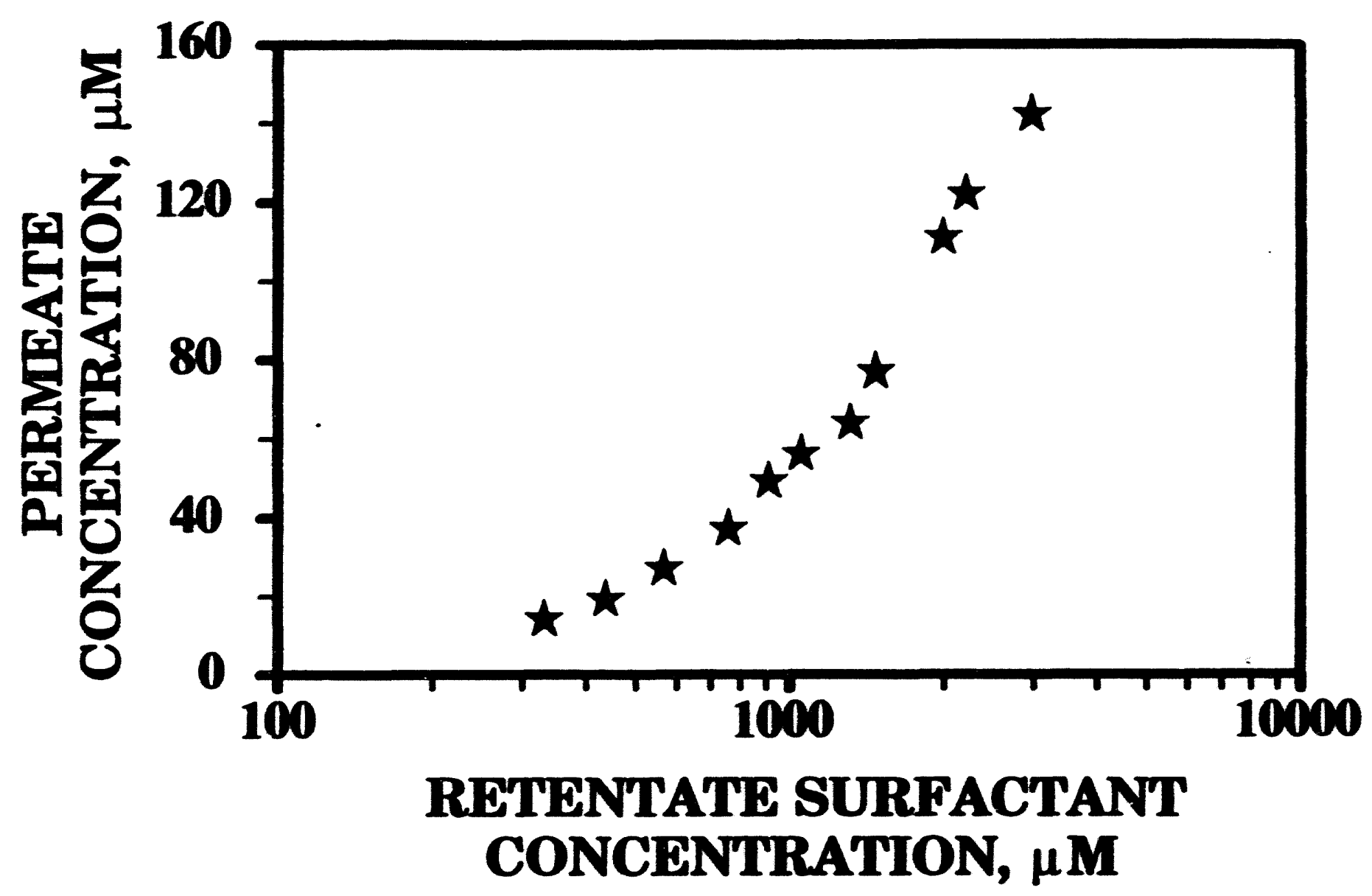

Figure VII.12: The Permeate Concentration for DOWFAX 8390 Using 500 MWCO

Spiral Wound Ultrafiltration Membrane 


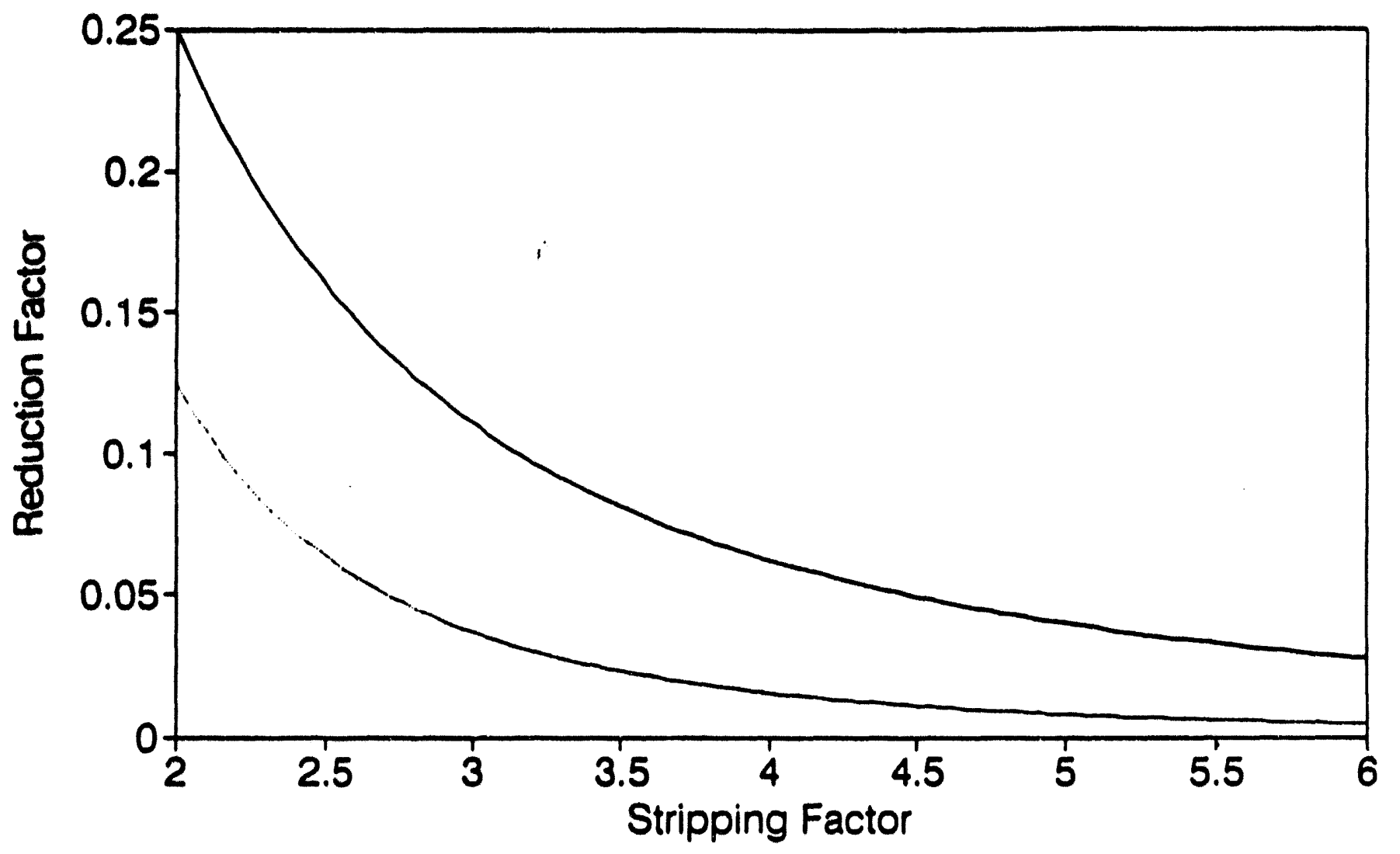

2 transfer units -3 transfer units

Figure VII.13: The Effect of the Number of Transfer Units and Stripping Factor on the Reduction Factor for TCE. 


\section{BIBLIOGRAPHY}

1. The Merck Index, (10th Ed.), Merck \& Co., Inc., Rathway, New Jersey, (1983), p.1378.

2. Kovach, J.L., "Gas Phase Adsorption", in Handbook of Separation Techniques for Chemical Engineers (P.A. Schweitzer, Ed.), Section 3.1, McGraw-Hill, New York (1979).

3. Hutchins, R.A., "Activated Carbon Systems for Separation of Liquids", in Handbook of Separations Techniques for Chemical Engineers (P.A. Schweitzer, Ed.), Section 1.13, McGraw-Hill, New York (1979).

4. Calgon Corporation, Adsorption Handbook, Activated Carbon Division-Calgon Corporation, Pittsburgh.

5. Soffel, R.W., "Carbon and Artificial Graphite", in KirkOthmer Encyclopedia of Chemical Technology, (3rd Ed.), Vol. 4, p.563, Wiley, New York (1983).

6. Scamehorn, J.F.; Ind. Fng. Chem. Process Des, Dey., 1979,18, 210.

7. Sutilkno, T.; Himmelstein, K.J.;Ind. Eng. Chem. Fundam., 1983, 822, 420.

8. Wankat, P.C.; Partin, L.C.; Ind Eng. Chem. Process Des. Dey., 1980, 19, 446.

9. Martin, R.J.; Ng, W.J.; Water Res, 1987, 21, 961.

10. Posey, R.J.; Kim, B.R.; J. Water Pollut. Control Fed. 1987, 59, 47.

11. Chudyk, W.A.; Snoeyink, V.L.;Environ. Sci. Technol., 1984, 18, 1. 
12. Dunn, Jr., R.O., Scamehorn, J.F., Christian, S.D.; Sep. Sci. Technol., 20, 257 (1985).

13. Scamehorn, J.F., Harwell, J.H., "An Overview of Surfactant Based Separations Processes", in The Role of Surfactants in Emerging Technology, (M.J. Rosen, Ed.), Ch. 8, Marcel Dekker, New York, (1987).

14. Harwell, J.H., Scamehorn, J.F., in Management of Hazardous and Toxic Wastes in the Process Industries, (Kolaczkowski, S.T., and Crittenden, B.D., Eds.),"Treatment of Hazardous and Toxic wastes Using Surfactant Based Separation Processes", Elsevier, London, (1987)

15. Scamehorn, J.F., Harwell, J.H., in Surfactants in Chemical Process Engineering, (D.T. Wasan, D.O. Shah, M.E. Ginn, Eds.), "Surfactant-Based Treatment of Aqueous Process Streams", Ch. 3, Marcel Dekker, New York, (1986).

16. Rosen, M.J.; "Surfactants and Interfacial Phenomena", (2nd Ed.), Ch. 3, Wiley, New York (1989).

17. Scamehorn, J.F., Ellington, R.T., Christian, S.D., Penny, B.W.,Dunn, R.O., Bhat, S.N., "Recent Advances In Separation Techniques - III, " (N. Li, Ed., T.A. Hatton, S. Hwang,D.M. LaRue, S.A. Leeper, D.L. Roberts, Co-Eds.), AIChE Symp. Ser. 82, 48 (1986).

18. Scamehorn, J.F., Christian, S.D., Ellington, R.T.,"Use of Micellar-Enhanced Ultrafiltration to Remove Multivalent Metal Ions from Aqueous Streams", in Surfactant-Based Separations Proceses, (Scamehorn, J.F., Harwell, J.H., Ed.) Ch. 2, Marcel Dekker, New York (1986).

19. Christian, S.D., Tucker, E.E., Scamehorn, J.F., El-Sayed, D.A., AIChE J, 34,189 (1988). 
20. Scamehorn, J.F., Christian, S.D., Ellington, R.T.,"Use of Micellar-Enhanced Ultrafiltration to Remove Dissolved Organics from Aqueous Streams", in Surfactant-Based Separations Proceses, (Scamehorn, J.F., Harwell, J.H., Ed.) Ch. 1, Marcel Dekker, New York (1986).

21. Dunn, R.O., Scamehorn J.F., Christian, S.D.; Sep. Sci. Technol., 22, 763 (1987).

22. Gibbs, L.L., Scamehorn, J.F., Christian, S.D.; J. Membrane Sci, 30, 67 (1987).

23. Hirasake, G.J., Lawson, J.B.; SPE Reservoir Eng, 1, 119 (1986).

24. Osborne-Lee, I.W., Schechter, R.S., Wade, W.H.; J.Colloid Inteface Sci., 94, 179 (1983).

25. Aboutaleb, A.E., Sakr, A.M., El-Sabbagh, H.M., Abdelrahman, S.I.; Pharm. Ind, 42, 940 (1980).

26 Aboutaleb, A.E., Sakr, A.M., El-Sabbagh, H.M., Abdelrahman, S.I.; Arch. Pharm. Chem. Sci. Ed. 5, 5, 105 (1977).

27. Blackburn, D.L., and Scamehorn, J.F., in Surfactant-Based Separation Processes, (Scamehorn, J.F., Harwell, J.H., Eds.), "Surfactant Enhanced Carbon Regeneration", Ch. 9, Marcel Dekker, New York, (1986).

28. Cheryan, M., Ultrafiltration Handbook, Technomic, Lancaster (1986).

29. Lonsdale, H.K., J. Membrane Sci., 10, 81 (1982).

30. Porter, M.C., in Handbook of Separation Techniques for Chemical Engineers, ( 2nd Ed.), (Schweitzer, P.A., Ed.) Sec. 2.1., McGraw-Hill, New York (1988).

31. Michaels, A.S., Chem. Eng. Prog, 64, 31 (1968). 
32. Welty, J.R., Wicks, C.E., Wilson, R.E., Fundamentals of Momentum, Heat, and Mass Transfer, p. 478, Wiley, New York (1976).

33. Welty, J.R., Wicks, C.E., Wilson, R.E., Fundamentals of Momentum, Heat, and Mass Transfer, p. 475, Wiley, New York (1976).

34. Choe, T.B., Masse, P., Verdier, A., Clifton, M.J., L Membrane Sci, 26, 1 (1986).

35. Nakao, S., Wijamans, J.G., Smolders, C.A., J. Membrane Sci., 26, 165 (1986).

36. Do, D.D., Elhassadi, A.A., J.Membrane Sci., 25, 113 (1985).

37. Dunn, Jr., R.O., Scamehorn, J.F., Christian, S.D., Sep. Sci. Technol, 20, 257 (1985).

38. Hiemenz, P.C., Principles of Colloid and Surface Chemistry, (2nd Ed.), Ch. 8, Marcel Dekker, New York (1986).

39. Nagarajan, R., Ruckenstein, E.; Sep Sci. Technol., 16, 1429 (1981).

40. Elworthy, P.H., Florence, A.T., MacFarlane, C.B., Solubilization by Surface-Active Agents, Ch. 2, Chapman and Hall, London (1968).

41. Weil, J.K., Smith, F.S., Stirton, A.J., Bristline, R.G., J.Am, Oil Chem. Soc., 40, 538 (1963).

42. Mukerjee, P., Solution Chemistry of Surfactants, Vol. 1, (K.L. Mittal, Ed.), p. 153, Plenum Press, New York (1979). 
43. Rosen, M.J., Surfactants and Interfacial Phenomena, Ch. 4, Wiley, New York (1978).

44. Christian, S.D., Smith, G.A., Tucker, E.E., Scamehorn, J.F., Lanemuir, 1, 564 (1985).

45. Tucker, E.E., Christian, S.D., J.Colloid Interface Sci., 104, 562 (1985).

46. Armstrong, D.W., Sep. Purif. Meth., 14, 213 (1985).

47. Arunyanart, M., Cline Love, L.J., Anal. Chem, 56, 1557 (1984).

48. Pramauro, E., Pelizzetti, E., Anal. Chem. Acta, 154, 153 (1983).

49. Armstrong, D.W., Nome, F., Anal Chem., 53, 1662 (1981).

50. Herries, D.G., Bishop, W., Richards, F.M., J.Phys, Chem., 68, 1842 (1964).

51. Yoesting, O.E., Scamehorn, J.F., Colloid and Polym. Sci, 264, 148 (1986).

52. Rathman, J.F., Scamehorn, J.F., J. Phys, Chem, 88, 5807 (1984).

53. Hiemenz, P.C., Principles of Colloid and Surface Chemistry, (2nd Ed.), Ch. 12, Marcel Dekker, New York (1986).

54. Mukerjee, P., Mysels, K.J., Kapauan, P., J. Phys. Chem., 71, 4116 (1967).

55. Oosawa, F., Polyelectrolytes, Marcel Dekker, New York (1971). 
56. El-Sayed, D.A., Scamehorn, J.F., Christian, S.D., Sep. Sci. Technnol, submitted for publication.

57. Lianos, R., Viriat, M.L., Zana, R., J. Phys, Chem, 1984, 88,1098.

58. Almgren, M., Grieser, F., Thomas, J.K., J.Am. Chem. Soc. 1979, 101, 279.

59. Roberts, B.L., Scamehorn, J.F., "Use of Surfactant-Enhanced Carbon Regeneration to Remove Volatile Organics from Spent Activated Carbon", in Surfactant-Based Separation Processes, (Scamehorn, J.F., Harwell, J.H., Ed.), Ch. 8, Marcel Deckker, New York, (1988).

60. Lafrance, P., Dusart, O., Mazet, M., 7 th International Symposium on Surfactants in Solution, Ottawa, Canada, 1988.

61. Schwuger, M.J., Smolka, H.G.; Colloid Polym. Sci., 1977, $25,589$.

62. Dunn, Jr., R.O., The Use of Micellar-Enhanced Ultrafiltration to Remove Dissolved Organics and Multivalent Transition Metal Cations From Aqueous Streams, Dissertation, University of Oklahoma (1987)

63. Smith, G.A., Christian, S.D., Tucker, E.E., and Scamehorn, J.F.; J. Colloid Interface Sci., 130, 254 (1989).

64. Nguyen, C.M., Christian, S.D., and Scamehorn, J.F.; Tenside Surfactant Deterg, 25, 328 (1988).

65. Nguyen, C.M., Scamehorn, J.F., and Christian, S.D.; Colloid Surf., 30, 335 (1988).

66. Rosen, M.J., Surfactants and Interfacial Phenomena, p. 99, Wiley, New York, 1978. 
67. Counce, R.M., Wilson, J.H., Singh, S.P., Ashworth, R.A., and Elliott, M.G.; ACS Symp. Ser., 468, 177 (1991).

68. Peters, M.S., and Timmerhaus, K.D., Plant Design and Economics for Chemical Engineers, (4th Ed.), McGrawHill, New York, (1991). 


\section{APPENDIX A.}

\section{Nomenclature}

(acronyms)

$\begin{array}{ll}\text { CHC } & =\text { chloronated hydrocarbon compounds } \\ \text { CPC } & =\text { cetylpyridinium chloride } \\ \text { CMC } & =\text { critical micelle concentration } \\ \text { DCM } & =\text { dichloromethane } \\ \text { DNP-18 } & =\text { polyoxyethylene (18) dinonyl phenol } \\ \text { MEUF } & =\text { micellar-enhanced ultrafiltration } \\ \text { MWCO } & =\text { molecular weight cut off } \\ \text { PCE } & =\text { perchloroethylene } \\ \text { ppb } & =\text { parts per billion } \\ \text { ppm } & =\text { parts per million } \\ \text { SDS } & =\text { sodium dodecyl sulfate } \\ \text { SED } & =\text { semi-equilibrium dialysis } \\ \text { SECR } & =\text { surfactant enhanced carbon regeneration } \\ \text { SHS } & =\text { sodium hexadecyl sulfate } \\ \text { STEDBAC } & =\text { stearyl dimethyl benzyl ammonium chloride } \\ \text { STS } & \text { sodium tetradecyl sulfate }\end{array}$




\section{Nomenclature (Continued) \\ (acronyms)}

$\begin{array}{ll}\text { TBP } & =\text { tert-4-butylphenol } \\ \text { TCE } & =\text { trichloroethylene } \\ \text { TCEA } & =1,1,1-\text { trichloroethane }\end{array}$

Nomenclature (Continued)

(variables)

$\alpha . \quad=$ adjustable empirical parameter

B = fraction of the micellar charge neutralized by counterions

$\phi \quad=$ fraction of the total volume within which the bound counterions are located

$\left[A S^{-}\right]_{\text {mon }}=$ anionic surfactant monomer concentration

$\left[A S^{-}\right]_{\text {mic }} \quad=$ anionic surfactant concentration in micellar form

$\left[A S^{-}\right]_{\text {per }} \quad=$ anionic surfactant concentration in the permeate

$\left[A S^{-}\right]_{\text {rew }} \quad=$ anionic surfactant concentration in the retentate

$\mathrm{C}_{\mathrm{b}} \quad=$ bulk retentate solute concentration

$\mathrm{C}_{\mathrm{g}} \quad=$ gel concentration

$\mathrm{C}_{\mathrm{m}} \quad=$ concentration of surfactant monomer

$\mathrm{C}_{8}=$ concentration of the organic solute solubilized 


\section{Nomenclature (Continued)}

(variables)

\begin{tabular}{|c|c|}
\hline $\mathrm{C}_{\mathbf{u}}$ & = concentration of unsolubilized organic solute \\
\hline$\left[\mathrm{Co}^{-}\right]_{\mathrm{ret}}$ & $\begin{array}{l}=\text { co-ion concentation of the divalent counterion } \\
\text { in the retentate }\end{array}$ \\
\hline$\left[\mathrm{Co}^{-}\right]_{\text {per }}$ & $\begin{array}{l}=\text { co-ion concentration of the divalent counterion in the } \\
\text { permeate }\end{array}$ \\
\hline $\mathbf{C}_{\mathrm{p}}$ & $=$ permeate solute concentration \\
\hline$\left[D^{2+}\right]_{b}$ & $\begin{array}{l}=\text { divalent counterion concentration bound to the micelle in } \\
\text { the retentate }\end{array}$ \\
\hline$\left[D^{2+}\right]_{u}$ & $\begin{array}{l}=\text { divalent counterion concentration not bound to the } \\
\text { micelle in the retentate }\end{array}$ \\
\hline$\left[D^{2+}\right]_{\text {rer }}$ & $=$ divalent counterion concentration in the retentate \\
\hline$\left[D^{2+}\right]_{\text {per }}$ & $=$ divalent counterion concentration in the permeate \\
\hline $\mathbf{D}_{\mathbf{a}, \mathbf{b}}$ & $=$ the diffusion coefficient of solute $\mathbf{a}$ in solvent $\mathbf{b}$ \\
\hline $\mathbf{J}_{\mathbf{w}}$ & $=$ solvent flux rate \\
\hline $\mathbf{J}_{\mathbf{a}, \mathbf{z}}$ & $=$ the molar flux of solute $\mathrm{a}$ in the $\mathrm{z}$ direction \\
\hline $\mathbf{K}$ & $=$ mass transfer coefficient of solute species in solvent \\
\hline $\mathbf{K}_{\mathbf{8}}$ & $=$ solubilization equilibrium constant \\
\hline $\mathbf{L}$ & $=$ molar flow rate of liquid through the stripping column \\
\hline$\left[M^{+}\right]_{b}$ & $\begin{array}{l}=\text { monovalent counterion concentration bound to the micelle } \\
\text { in the retentate }\end{array}$ \\
\hline
\end{tabular}




\section{Nomenclature (Continued) \\ (variables)}

$\left[\mathrm{M}^{+}\right]_{u}=$ monovalent counterion concentration not bound to the micelle in the retentate

$\left[M^{+}\right]_{\text {res }} \quad$ = monovalent counterion concentration in the retentate

$\left[M^{+}\right]_{\text {per }}=$ monoalent counterion concentration in the permeate

$\left[\mathrm{M}^{+} \mathrm{Co}^{-}\right]=$concentration of the added monovalent electrolyte, the $M^{+}$cation is the same as that from the salt of the anionic surfactant and the $\mathrm{Co}^{-}$anion is the same as that from the salt of the multivalent cation.

Ntr = number of overall transfer units for stripping column

$P \quad=$ dimensionless electrical potential intensity parameter

Po = dimensionless intensity parameter in micellar solution containing no added monovalent electrolyte

$R_{b} \quad=$ bulk retentate solution rejection coefficient

$R_{c} \quad=$ cake resistance to flux

Rf = reduction factor of the volatile compound in solution for the stripping column

$R_{m} \quad=$ membrane resistance to flux

$R_{t} \quad=$ true rejection coefficient

S $\quad=$ stripping factor

$\mathrm{V}=$ molar flow rate of vapor through the stripping column 
Nomenclature (Continued)

(variables)

$\mathrm{X}_{\mathrm{TCE}} \quad=$ mole fraction of TCE in the micelle 

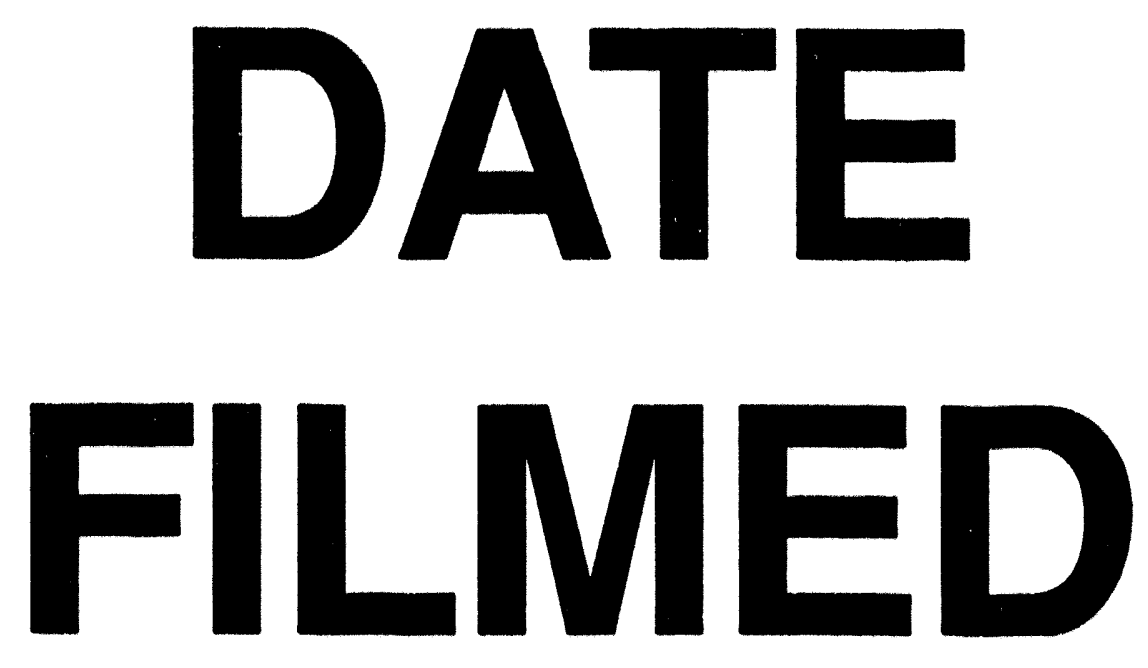

$6 / 3 / 94$
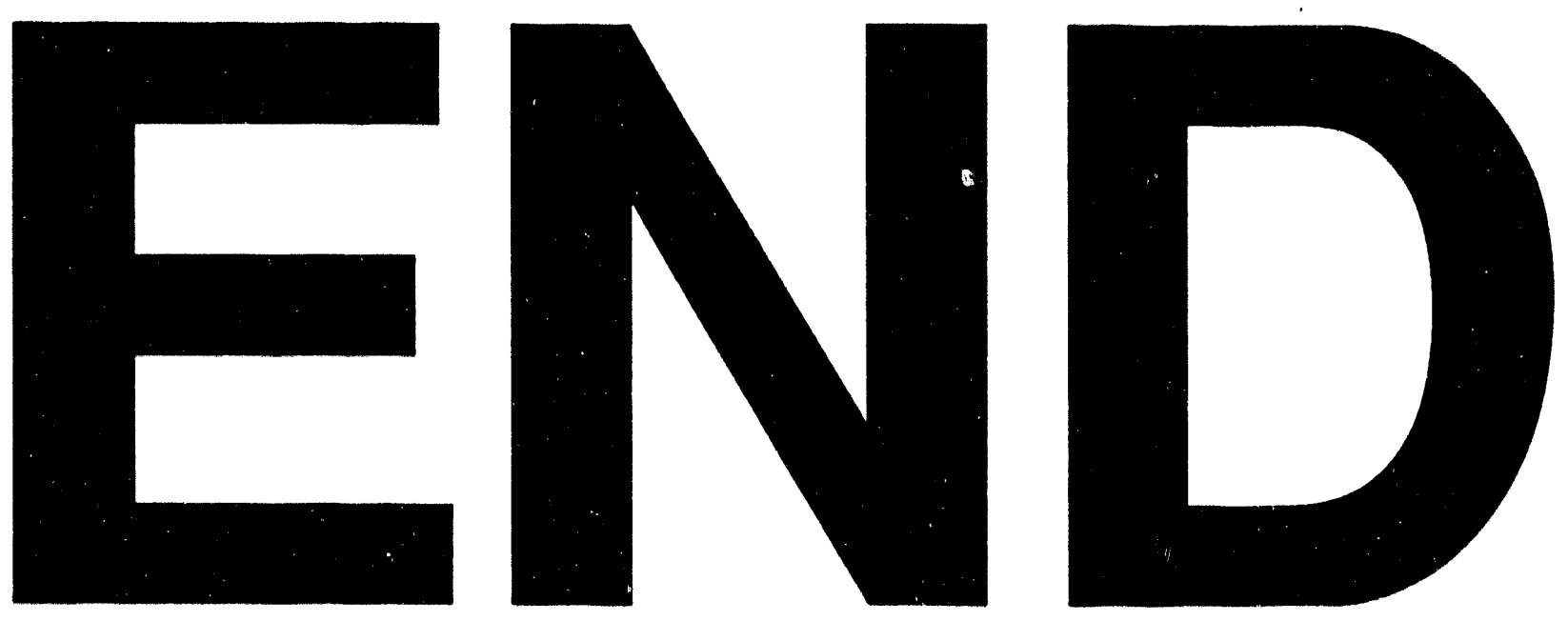
